Florida International University FIU Digital Commons

3-23-2010

\title{
The Development of High Performance Liquid Chromatography Systems for the Analysis of Improvised Explosives
}

Megan N. Bottegal

Florida International University, Megan.Bottegal@usss.dhs.gov

DOI: $10.25148 /$ etd.FI10041603

Follow this and additional works at: https://digitalcommons.fiu.edu/etd

\section{Recommended Citation}

Bottegal, Megan N., "The Development of High Performance Liquid Chromatography Systems for the Analysis of Improvised Explosives" (2010). FIU Electronic Theses and Dissertations. 154.

https://digitalcommons.fiu.edu/etd/154 


\title{
FLORIDA INTERNATIONAL UNIVERSITY
}

Miami, Florida

\section{THE DEVELOPMENT OF OPTIMIZED HIGH PERFORMANCE LIQUID CHROMATOGRAPHY SYSTEMS FOR THE ANALYSIS OF IMPROVISED EXPLOSIVES}

\author{
A dissertation submitted in partial fulfillment of the \\ requirements for the degree of \\ DOCTOR OF PHILOSOPHY \\ in \\ CHEMISTRY \\ by \\ Megan Nicole Bottegal
}

2010 
To: Dean Kenneth Furton

College of Arts and Sciences

This dissertation, written by Megan Nicole Bottegal, and entitled The Development of Optimized High Performance Liquid Chromatography Systems for the Anlysis of Improvised Explosives, having been approved in respect to style and intellectual content, is referred to you for judgment.

We have read this dissertation and recommend that it be approved.

$\begin{array}{r}\hline \text { Jose Almirall } \\ \hline \text { John Berry } \\ \hline \text { William Hearn }\end{array}$

William Hearn

Fenfei Leng

DeEtta Mills

Bruce McCord, Major Professor

Date of Defense: March 23, 2010

The dissertation of Megan Nicole Bottegal is approved.

\begin{tabular}{r} 
Dean Kenneth Furton \\
College of Arts and Sciences \\
\hline Interim Dean Kevin O'Shea \\
University Graduate School
\end{tabular}

Florida International University, 2010 
C Copyright 2010 by Megan Nicole Bottegal

All rights reserved. 


\section{DEDICATION}

I dedicate this dissertation to my parents. Without their love, patience, generosity, and guidance, the completion of this work would have been impossible. 


\section{ACKNOWLEDGMENTS}

I would like to thank the members of my dissertation committee for their support and assistance. Dr. Bruce McCord granted me the opportunities to conduct interesting research, to learn new analytical techniques, and to attend a variety of conferences and meetings. Dr. Jose Almirall first encouraged me to attend Florida International University, and brought a critical eye to all project discussions. Dr. John Berry provided helpful editorial feedback as well as a compassionate listening ear. Dr. Lee Hearn provided much-needed materials and ideas. Dr. Fenfei Leng provided valuable suggestions regarding method validation. Dr. DeEtta Mills rendered assistance with project planning and time management.

I would also like to thank the people outside of Florida International University who provided invaluable assistance and support. Dr. Lisa Lang of the BATFE Laboratory provided me with instrument access, guidance, and friendship. Dr. Mark Miller of the FBI Laboratory granted me a wonderful opportunity to work as a Visiting Scientist. My experiences in that program were excellent. Kelly Mount and Dr. Kirk Yeager of the FBI Laboratory provided many of the post-blast samples for this project, as well as unforgettable moments on the demolition range. 


\section{ABSTRACT OF THE DISSERTATION \\ THE DEVELOPMENT OF OPTIZIMED HIGH PERFORMANCE LIQUID CHROMATOGRAPHY SYSTEMS FOR THE ANALYSIS OF IMPROVISED EXPLOSIVES}

by

\section{Megan Nicole Bottegal}

Florida International University, 2010

Miami, Florida

\section{Professor Bruce McCord, Major Professor}

Existing instrumental techniques must be adaptable to the analysis of novel explosives if science is to keep up with the practices of terrorists and criminals. The focus of this work has been the development of analytical techniques for the analysis of two types of novel explosives: ascorbic acid-based propellants, and improvised mixtures of concentrated hydrogen peroxide/fuel. In recent years, the use of these explosives in improvised explosive devices (IEDs) has increased. It is therefore important to develop methods which permit the identification of the nature of the original explosive from postblast residues.

Ascorbic acid-based propellants are low explosives which employ an ascorbic acid fuel source with a nitrate/perchlorate oxidizer. A method which utilized ion chromatography with indirect photometric detection was optimized for the analysis of intact propellants. Post-burn and post-blast residues if these propellants were analyzed. It was determined that the ascorbic acid fuel and nitrate oxidizer could be detected in intact 
propellants, as well as in the post-burn and post-blast residues. Degradation products of the nitrate and perchlorate oxidizers were also detected.

With a quadrupole time-of-flight mass spectrometer (QToFMS), exact mass measurements are possible. When an HPLC instrument is coupled to a QToFMS, the combination of retention time with accurate mass measurements, mass spectral fragmentation information, and isotopic abundance patterns allows for the unequivocal identification of a target analyte. An optimized HPLC-ESI-QToFMS method was applied to the analysis of ascorbic acid-based propellants. Exact mass measurements were collected for the fuel and oxidizer anions, and their degradation products. Ascorbic acid was detected in the intact samples and half of the propellants subjected to open burning; the intact fuel molecule was not detected in any of the post-blast residue.

Two methods were optimized for the analysis of trace levels of hydrogen peroxide: HPLC with fluorescence detection (HPLC-FD), and HPLC with electrochemical detection (HPLC-ED). Both techniques were extremely selective for hydrogen peroxide. Both methods were applied to the analysis of post-blast debris from improvised mixtures of concentrated hydrogen peroxide/fuel; hydrogen peroxide was detected on variety of substrates. Hydrogen peroxide was detected in the post-blast residues of the improvised explosives TATP and HMTD. 


\section{TABLE OF CONTENTS}

CHAPTER

PAGE

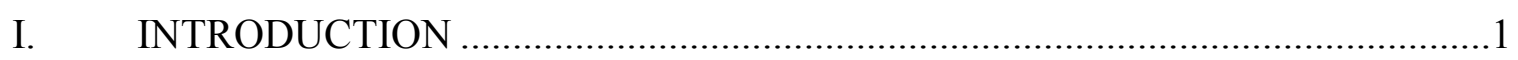

Forensic Analysis of Explosives ...................................................................

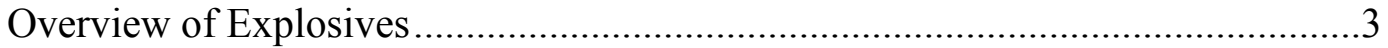

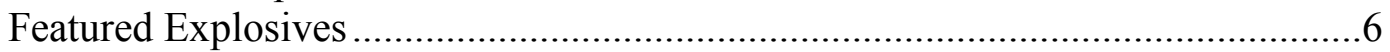

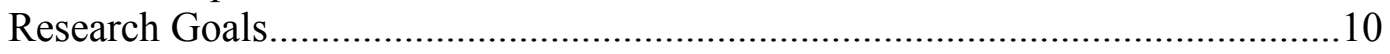

II. INSTRUMENTAL ANALYSIS OF EXPLOSIVES........................................12

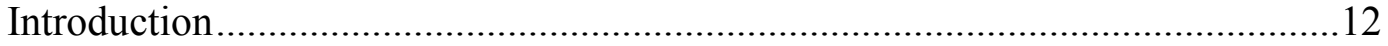

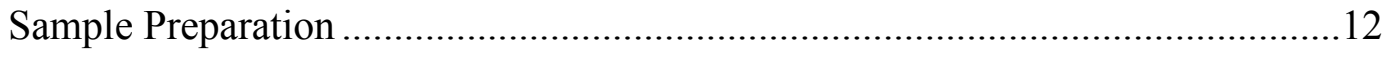

High Performance Liquid Chromatography .......................................................14

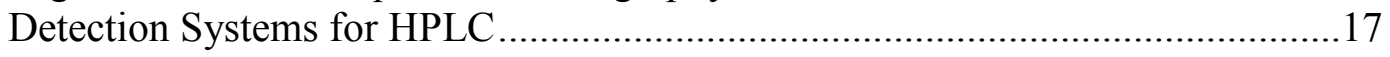

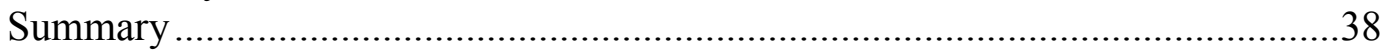

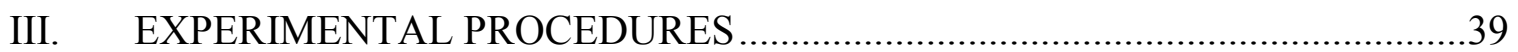

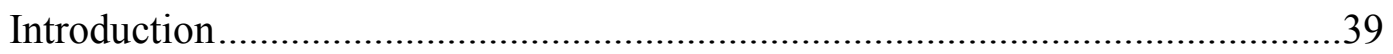

Analysis of Ascorbic Acid-Based Propellants by IC-IPD ......................................40

Analysis of Ascorbic Acid-Based Propellants by HPLC-ESI-QToFMS................43

Analysis of Hydrogen Peroxide by HPLC-ED and HPLC-FD ..............................48

IV. ANALYSIS OF ASCORBIC ACID-BASED PROPELLANTS BY IC-IPD .......58

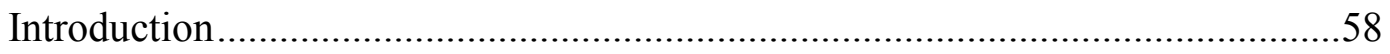

Development of IC-IPD Method ..................................................................67

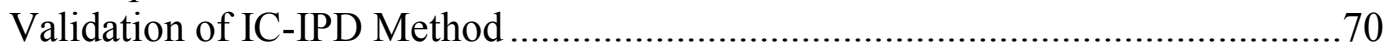

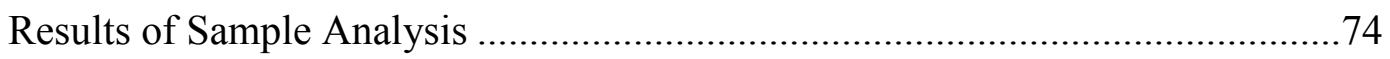

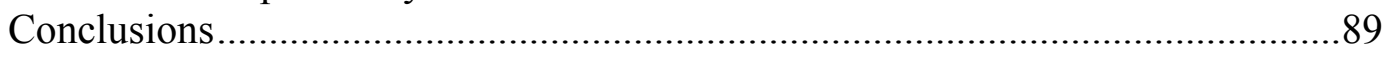

V. ANALYSIS OF ASCORBIC ACID-BASED PROPELLANTS BY

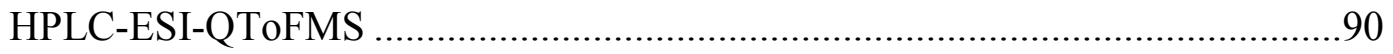

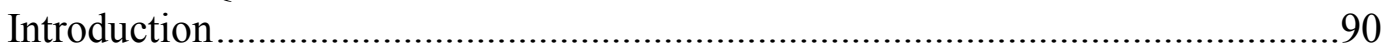

Development of HPLC-ESI-QToFMS Method .....................................................92

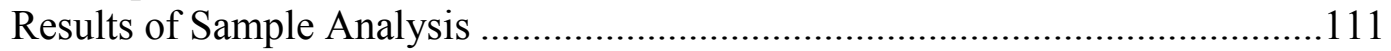

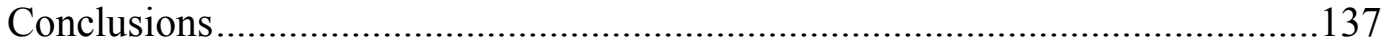

VI. OPTIMIZATION OF HPLC-ED AND HPLC-FD METHODS FOR THE

ANALYSIS OF HYDROGEN PEROXIDE ...............................................139

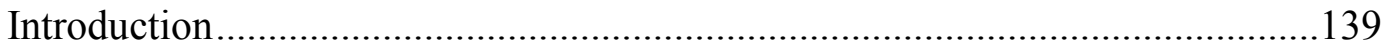

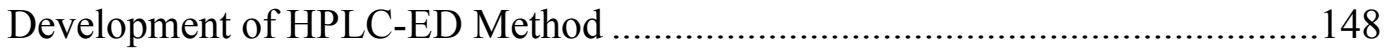

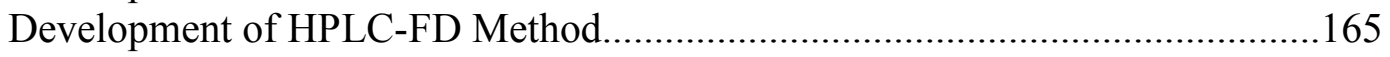

Validation of HPLC-ED and HPLC-FD Methods .............................................178

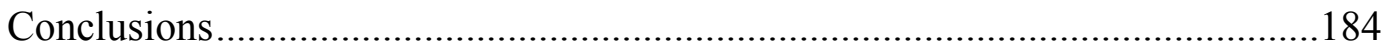


VII. ANALYSIS OF FIELD SAMPLES BY HPLC-ED AND HPLC-FD ...............185

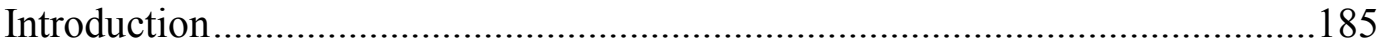

Effect of Mode of Application, Storage, and Extraction on the Recovery of $\mathrm{H} 2 \mathrm{O} 2$

Evaluation of Cotton Sampling Media

Survey of Law Enforcement Training Facility

.202

Analysis of Post-Blast Debris from Mixtures of Concentrated

Hydrogen Peroxide and Nitromethane .205

Analysis of Organic Peroxides.

Analysis of TATP and HMTD

Conclusions .238

VIII. CONCLUSIONS.

LIST OF REFERENCES 


\section{LIST OF TABLES}

TABLE

PAGE

3.1 QToFMS Parameters for the Analysis of Anion Standards and

Propellant Samples. .46

4.1 Composition of Propellants Utilized in this Study and their Expected Post-Blast Anionic Residues

4.2 Figures of Merit for IC-IPD.

4.3 Detection Limit and Linear Dynamic Range for Organic and Inorganic Anions by IC-IPD

4.4 Results of Analysis of Intact Propellant Samples ............................................76

4.5 Results of Analysis of Burned Propellant Samples ....................................... 80

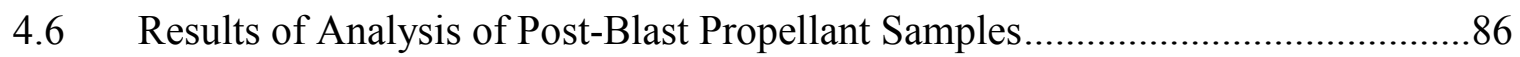

5.1 Nominal and Exact Masses of Analytes of Interest .......................................93

5.2 Accurate Mass Data for Anion Standards by HPLC-ESI-QToFMS ..................102

5.3 Figures of Merit for Anion Standards by HPLC-ESI-QToFMS ........................102

5.4 Accurate Mass Data for Nitrate and Nitrate Cluster .......................................106

5.5 Elemental Composition Report from MassLynx Software Regarding

Identity of Ascorbate and the Fragment Ions

5.6 Composition of Black Powder Substitutes

5.7 Accurate Mass Data for the Anions Present in Intact Grains of American Pioneer.

5.8 Accurate Mass Data for the Anions Present in Intact Grains of Goex Pinnacle Replica

5.9 Accurate Mass Data for the Anions Present in Intact Grains of Black Canyon

5.10 Results of Analysis of Intact Propellant Samples by HPLC-ESI-QToFMS 
5.11 Accurate Mass Data for the Anions Present in Post-Burn Residues of Black Mag3

5.12 Accurate Mass Data for the Anions Present in Post-Burn Residues of Clean Shot.

5.13 Results of Analysis of Post-Burn Residues by HPLC-ESI-QToFMS

5.14 Accurate Mass Data for the Anions Present in Post-Blast Residues from Shot III

5.15 Accurate Mass Data for the Anions Present in Post-Blast Residues from Shot V

5.16 Results of Analysis of PVC Fragments by HPLC-ESI-QToFMS

6.1 Effect of Temperature of the Electrochemical Detector Flow Cell on the Peak Area of Hydrogen Peroxide

6.2 Effect of PAD Settings on Detection of a Hydrogen Peroxide Standard 156

6.3 Effect of New Mobile Phase Concentration on Retention Time and Peak Area Of Hydrogen Peroxide.

6.4 Effect of Analytical Column on Retention Time and Peak Area of

$0.5 \mathrm{ppm}$ Hydrogen Peroxide 166

6.5 Effect of Mobile Phase Flow Rate on Peak Area and Retention Time of $0.5 \mathrm{ppm}$ Hydrogen Peroxde

6.6 Effect of Acetonitrile on Detection of $0.5 \mathrm{ppm}$ Hydrogen Peroxide

6.7 Effect of Length and Inner Diameter of Reaction Tubing on Detection Of 0.5 ppm Hydrogen Peroxide.

6.8 Effect of Temperature of the Reaction Tubing on Detection of $0.5 \mathrm{ppm}$ Hydrogen Peroxide

6.9 Effect of Concentration of Sodium Hydroxide on Detection of $0.5 \mathrm{ppm}$

Hydrogen Peroxide

6.10 Evaluation of Additional Excitation/Emission Wavelength Pairs to Optimize Detection of Hydrogen Peroxide.

6.11 Effect of the Presence of $100 \mu \mathrm{M}$ of an Inorganic Anion on the Peak Area of $100 \mu \mathrm{M} \mathrm{H}_{2} \mathrm{O}_{2}$ 
6.12 Reproducibility Data for the Retention Time and Peak Area of $0.5 \mathrm{ppm}$ And 5.0 ppm Hydrogen Peroxide

7.1 Mode of Application versus Recovery of Hydrogen Peroxide Residue

7.2 Effect of Mode of Extraction on Recovery of Hydrogen Peroxide Residue

7.3 Recovery of Hydrogen Peroxide from Four Different Substrates

7.4 Results of Analysis of Seven Types of Blank Cotton Swabs

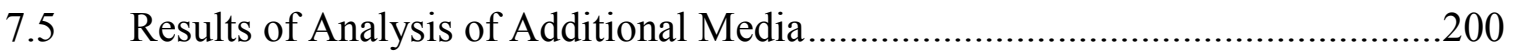

7.6 Results of a Survey of a Law Enforcement Training Academy ..........................204

7.7 Results of Analysis of Post-Blast Debris from a Large-Scale Detonation Of Hydrogen Peroxide/Nitromethane .206

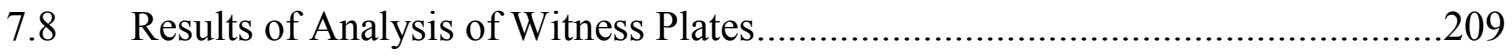

7.9 Results of Re-Analysis of Five Witness Plate Extracts .......................................212

7.10 Results of Initial Analysis of Materials Collected from a Small-Scale Detonation of Hydrogen Peroxide/Nitromethane Inside of an Ammo Can

7.11 Results of Secondary Analysis of Materials Collected from a Small-Scale Detonation of Hydrogen Peroxide/Nitromethane Inside of an Ammo Can

7.12 Results of Analysis of Materials Collected from a Small-Scale Detonation Of Hydrogen Peroxide/Nitromethane Inside of a Car .218

7.13 Results of Analysis of Materials Collected from a Second Small-Scale Detonation of Hydrogen Peroxide/Nitromethane Inside of an Ammo Can

7.14 Results of Testing Organic Peroxides without the Presence of the Analytical Column. .228 


\section{LIST OF FIGURES}

FIGURE

PAGE

2.1 Normal Phase Silica and Reversed Phase C-18 Silica......................................16

2.2 Energy Diagram of the Fluorescence Process ...............................................21

2.3 Three Electrode Configuration for a Standard Amperometric Flow Cell

Used in Electrochemical Detection............................................................2

2.4 One Cycle of Pulsed Amperometric Detection................................................29

2.5 Schematic of a Hybrid Quadrupole Time-of-Flight Mass Spectrometer...............35

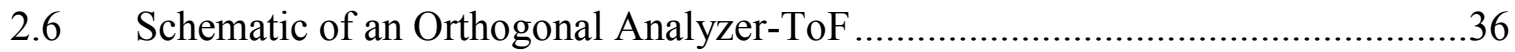

2.7 Plot of the Asynchronous Duty Cycle of an oa-ToF versus an Enhanced

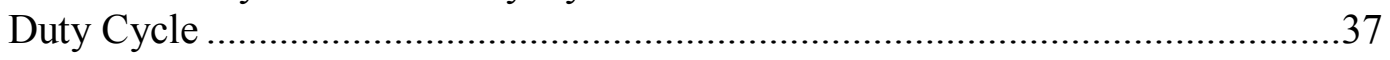

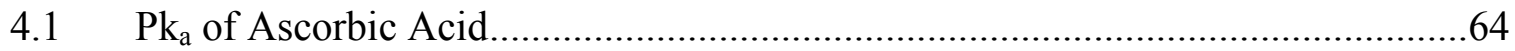

4.2 Degradation Process of Ascorbic Acid Resulting in the Formation of

2,3-Diketogulonic Acid, Oxalic Acid, and Threonic Acid

4.3 Pathway for the Formation of Diketogulonic acid from Ascorbic Acid...............66

4.4 Oxidation of Ascorbic Acid to Dehydroascorbic Acid and Oxidation

Of Cysteine to Cystine.

4.5 Separation of a Standard Anion Mixture by Ion Chromatography .......................68

4.6 Degradation of Ascorbic Acid Over Time With and Without the

Addition of an Excess of Cysteine, as Measured by IC-IPD

4.7 Calibration Curve Data for Ascorbate by IC-IPD …........................................ 71

4.8 Calibration Curve Data for Chloride, Nitrite, Nitrate, Chlorate, and Sulfate by IC-IPD.

4.9 Analysis of Intact American Pioneer Powder ..................................................75

4.10 Analysis of Intact Jim Shockey’s Gold Powder ..........................................75

4.11 Analysis of Burned Jim Shockey’s Gold Powder ...........................................78 
4.12 Analysis of Burned American Pioneer Powder ...................................................78

4.13 Analysis of Burned Triple7 Powder ..................................................................

4.14 Relative Abundance of Residues Present in Burned Propellant Samples..............82

4.15 Analysis of Post-Blast American Pioneer Pipe Shot ..............................................8

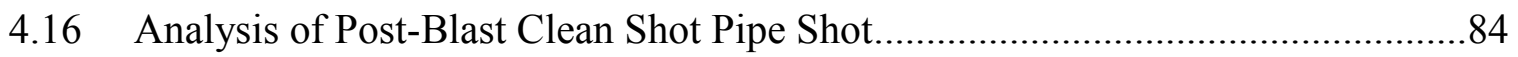

4.17 Analysis of Post-Blast Black Mag3 Pipe Shot.....................................................85

4.18 Relative Abundance of Residues Present in Post-Blast Propellant Samples..........88

5.1 Analysis of a Standard Solution of Ascorbic Acid, Nitrite, Chlorate, Nitrate, and Perchlorate by HPLC-ESI-QToFMS 100

5.2 Analysis of the Unknown Ion at $m / z 209.9409$ by HPLC-ESI-QToFMS-MS With Collision Energy $15 \mathrm{eV}$

5.3 Isotopic Distribution of $\left[\mathrm{NO}_{3} \mathrm{NO}_{3}{ }^{-} \mathrm{NO}_{2}{ }^{-} \mathrm{H}^{+} \mathrm{K}^{+}\right]$Cluster at $\mathrm{m} / z$ 209.9401 .............106

5.4 Degradation Process of Ascorbic Acid to Yield 2,3-Diketogulonic Acid, Threonic Acid, and Oxalic Acid

5.5 Mass Spectrum from the Analysis of Ascorbate by HPLC-ESI-QToFMS

5.6 Alternative Degradation Pathway for Ascorbate to Yield Fragment Ions at $\mathrm{m} / \mathrm{z} 115.0026$ and $\mathrm{m} / \mathrm{z} 87.0083$.

5.7 Analysis of Intact Grains of American Pioneer by HPLC-ESI-QToFMS ...........113

5.8 Analysis of Intact Grains of Goex Pinnacle Replica by HPLC-ESI-QToFMS

5.9 Analysis of Intact Grains of Black Canyon by HPLC-ESI-QToFMS ……..........118

5.10 Analysis of Post-Burn Residues from Black Mag3 by HPLC-ESI-QToFMS .....121

5.11 Analysis of Post-Burn Residues from Clean Shot by HPLC-ESI-QToFMS .......125

5.12 Analysis of PVC Fragments from Shot III by HPLC-ESI-QToFMS ..................129

5.13 Analysis of PVC Fragments from Shot V by HPLC-ESI-QToFMS …………....133 
6.1 Effects of Analytical Column, Mobile Phase, and Flow Rate on the Peak Shape and Retention Time of 300 ppm Hydrogen Peroxide.

6.2 Effect of Mobile Phase Concentration on Peak Shape and Retention Time of 0.5 ppm Hydrogen Peroxide Standard

6.3 Analysis of a 0.5 ppm Hydrogen Peroxide Standard by HPLC-ED In PAD Mode.

6.4 Theoretical Hydrodynamic Voltammogram for Hydrogen Peroxide .161

6.5 Hydrodynamic Voltammogram of Hydrogen Peroxide in $150 \mathrm{mM} \mathrm{NaOAc}$

6.6 Analysis of 0.5 ppm Hydrogen Peroxide by HPLC-ED in DC Mode 163

6.7 Analysis of 0.5 ppm Hydrogen Peroxide by HPLC-FD .......................................166

6.8 Effect of Changing Emission Wavelength on Detection of Hydrogen Peroxide

6.9 Effect of Changing Excitation Wavelength on Detection of Hydrogen Peroxide

6.10 Analysis of a 0.5 ppm Hydrogen Peroxide Standard by HPLC-FD .....................176

6.11 Calibration Curve for Hydrogen Peroxide on the HPLC-FD System At Standard Conditions: $0.5 \mathrm{ppm}-10.0 \mathrm{ppm}$

6.12 Calibration Curve for Hydrogen Peroxide on the HPLC-ED System In DC Mode at Standard Conditions: $0.25 \mathrm{ppm}-500 \mathrm{ppm}$

7.1 Analysis of a Wal-Mart Cotton Ball by HPLC-ED in DC Mode ..........................196

7.2 Analysis of a Wal-Mart Cotton Ball by HPLC-FD .............................................197

7.3 Analysis of a Cotton Swatch from a Roll of Cotton by HPLC-ED in DC Mode.

7.4 Analysis of a Cotton Swatch from a Roll of Cotton by HLC-FD 198

7.5 Analysis of a Metal Debris Fragment by HPLC-ED in PAD Mode....................206

7.6 Analysis of a Metal Debris Fragment by HPLC-FD ...........................................207 
7.7 Analysis of a Metal Witness Plate Exposed to Concentrated Hydrogen

Peroxide/Fuel \#1 by HPLC-FD

7.8 Analysis of a Metal Witness Plate Exposed to Concentrated Hydrogen

Peroxie/Fuel \#1 by HPLC-ED in PAD Mode.

7.9 Analysis of a Metal Witness Plate Exposed to Concentrated Hydrogen

Peroxide/Fuel \#1 by HPLC-ED in DC Mode

7.10 Analysis of a Plastic Remnant by HPLC-ED in DC Mode …………………....215

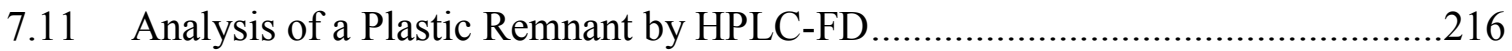

7.12 Analysis of a Water Rinse of Exterior of Ammunition Can Debris by HPLC-ED in DC mode

7.13 Analysis of a Water Rinse of Exterior of Ammunition Can Debris by HPLC-FD.

7.14 Analysis of a Witness Plate Exposed to a Small-Scale Detonation of Hydrogen Peroxide/Nitromethane by HPLC-ED in DC Mode .220

7.15 Analysis of a Witness Plate Exposed to a Small-Scale Detonation of Hydrogen Peroxide/Nitromethane by HPLC-FD

7.16 Analysis of a Plastic Remant by HPLC-ED in DC Mode .222

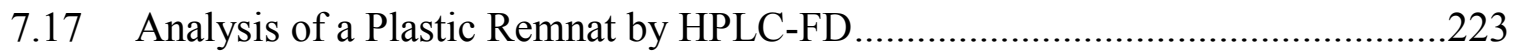

7.18 Analysis of $1^{\text {st }}$ Dry Swab of Interior of Ammunition Can by HPLC-ED in DC Mode.

7.19 Analysis of $1^{\text {st }}$ Dry Swab of Interior of Ammunition Can by HPLC-FD .............225

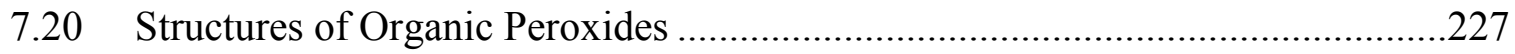

7.21 Fluorescence Response to Peroxides from Strongest to Weakest ........................229

7.22 Electrochemcal Response to Peroxides from Strongest to Weakest.....................230

7.23 Analysis of Peroxides by Electrochemical Detection in PAD Mode ...................231

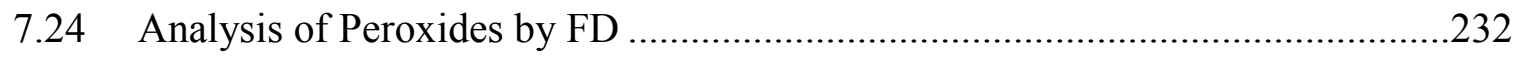

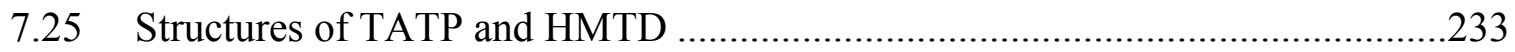




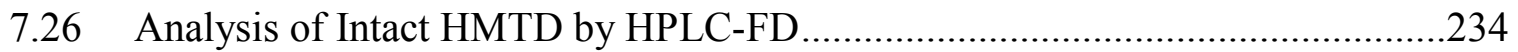

7.27 Analysis of Intact HMTD by HPLC-ED in DC Mode ...................................235

7.28 Analysis of Post-Blast Residues of TATP by HPLC-ED in DC Mode ...............236

7.29 Analysis of Post-Blast Residues of TATP by HPLC-FD .................................236

7.30 Analysis of Post-Blast Residues of HMTD by HPLC-ED in DC Mode ............237

7.31 Analysis of Post-Blast Residues of HMTD by HPLC-FD ...............................238 


\section{LIST OF ABBREVIATIONS AND ACRONYMS}

ANFO: Ammonium Nitrate Fuel Oil

APCI: Atmospheric Pressure Chemical Ionization

API: Atmospheric Pressure Ionization

ATF: Bureau of Alcohol, Tobacco, and Firearms

BSA: bis(trimethylsilyl)acetamide

CE: Capillary Electrophoresis

CE-IPD: Capillary Electrophoresis-Indirect Photometric Detection

CE-UV: Capillary Electrophoresis-Ultraviolet Detection

cm: centimeter

DAD: Diode Array Detector

DC: Direct Current

DETA: Diethylene Triamine

DI: De-ionized

EDTA: Ethylenediaminetetracetic Acid

EDX: Energy Dispersive X-Ray Spectroscopy

EI: Electron Impact

EOF: Electroosmotic Flow

ESI: Electrospray Ionization

ESI-MS: Electrospray Ionization-Mass Spectrometry

FBI: Federal Bureau of Investigation

FTIR: Fourier Transform Infrared Spectroscopy 
g: Gram

GC: Gas Chromatography

GC-ECD: Gas Chromatography-Electron Capture Detector

GC-MS: Gas Chromatography-Mass Spectrometry

HDB: Hexadimethrine Bromide

HMX: Cyclotetramethylene-tetranitramene

HMTD: Hexamethylene Triperoxide Diamine

$\mathrm{H}_{2} \mathrm{O}_{2}$ : Hydrogen Peroxide

HPLC: High Performance Liquid Chromatography

HPLC-ED: High Performance Liquid Chromatography-Electrochemical Detection

HPLC-ESI-MS: High Performance Liquid Chromatography-Electrospray IonizationMass Spectrometry

HPLC-FD: High Performance Liquid Chromatography-Fluorescence Detection

HPLC-UV: High Performance Liquid Chromatography-Ultraviolet Detection

HPLC-MS: High Performance Liquid Chromatography-Mass Spectrometry

$\mathrm{H}_{2} \mathrm{SO}_{4}$ : Sulfuric Acid

Hz: Hertz

I/E: Current/Potential

IC: Ion Chromatography

IC-CD: Ion Chromatography-Conductivity Detection

IC-IPD: Ion Chromatography-Indirect Photometric Detection

IC-MS: Ion Chromatography-Mass Spectrometry

ICUMSA: International Commission for Uniform Measures for Sugar Analysis 
IC-UV: Ion Chromatography-Ultraviolet Detection

ID: Inner Diameter

IED: Improvised Explosive Device

IMS: Ion Mobility Spectrometry

$\mathrm{KClO}_{3}$ : Potassium Chlorate

$\mathrm{KClO}_{4}$ : Potassium Perchlorate

$\mathrm{KNO}_{2}$ : Potassium Nitrite

$\mathrm{KNO}_{3}$ : Potassium Nitrate

KOH: Potassium Hydroxide

$\mathrm{kV}$ : Kilovolt

$\mathrm{L}_{\text {teff: }}$ : Effective Length of Flight Tube

LOD: Limit of Detection

M: Molar

MeCN: Acetonitrile

$\mu \mathrm{g}:$ Microgram

$\mu \mathrm{L}:$ Microliter

$\mu \mathrm{m}$ : Micrometer

$\mu \mathrm{M}$ : Micromolar

$\mu \mathrm{s}:$ Microsecond

MS/MS: Mass Spectrometry-Mass Spectrometry

$\mathrm{mV}$ : Millivolt

$m / z$ : Mass to Charge Ratio

NaOAc: Sodium Acetate 
$\mathrm{NaOH}$ : Sodium Hydroxide

$\mathrm{NH}_{4} \mathrm{Cl}$ : Ammonium Chloride

$\mathrm{NH}_{4} \mathrm{OH}$ : Ammonium Hydroxide

nm: Nanometer

PAD: Pulsed Amperometric Detection

PETN: Pentaerythritol Tetranitrate

POPHA: Para-Hydroxyphenylacetic Acid

ppb: Parts-Per-Billion

ppm: Parts-Per-Million

psi: Pounds per Square Inch

PVC: Poly-Vinyl Chloride

QToF: Quadrupole Time-of-Flight

RDX: Cyclotrimethylenetrinitramene

RF: Radio Frequency

RSD: Relative Standard Deviation

$\mathrm{R}_{\mathrm{t}}$ : Retention Time

s: Seconds

SPE: Solid Phase Extraction

TATP: Triacetone Triperoxide

ToF: Time-of-Flight

ToFMS: Time-of-Flight Mass Spectrometer

TNT: Trinitrotoluene

UPLC: Ultra Performance Liquid Chromatography 
UV-VIS: Ultraviolet-Visible

V: Volt

Wt: Weight

XRD: X-Ray Diffraction 


\section{Chapter I. Introduction}

\section{A. Forensic Analysis of Explosives}

Explosives have a variety of applications, including (but not limited to) mining and quarrying, demolition, construction, avalanche control, small arms fire, pyrotechnic displays and rocketry. A considerable volume of explosives are consumed every year. For example, in 2007, approximately 3.15 million metric tons of industrial explosives and blasting agents were sold in the United States for use in mining and construction (1). Explosives are also used by terrorists and other criminal organizations. The National Counterterrorism Center documented the use of explosives in more than 4,500 terrorist acts worldwide which left thousands of people dead or injured in all of 2007 (2). Many of these attacks utilized improvised explosive devices (IEDs). Homemade IEDs used by terrorists are often based on commercial gun powders, inorganic salts, and/or peroxides. These materials, or their starting components, are readily available and are of low cost (3).

When an explosive incident of a criminal nature occurs, forensic investigators seek to answer questions regarding the type of explosive used. This task may require identifying the main charge of an explosive device, confirming the identity of known bulk explosives, identifying unknown bulk explosives, or identifying residues of explosives. For legal purposes, "identification" of an unknown material must incorporate results obtained from presumptive and confirmatory testing. Generally, presumptive testing is relatively non-specific, involving such tests as color reactions. In contrast, confirmatory testing uses a different, more selective analytical method than a screening test. Confirmatory testing may involve laboratory based techniques such as high 
performance liquid chromatography (HPLC), gas chromatography (GC), mass spectrometry (MS), and/or spectroscopic techniques. Essentially, confirmatory testing must utilize techniques which provide significant structural and/or elemental information about the compound of interest (4). One approach to confirmatory testing involves the combination of chromatographic techniques with a selective detector, such as high performance liquid chromatography-mass spectrometry (HPLC-MS).

A typical protocol for the analysis of post-blast explosives debris involves the analysis of particulate matter followed by extraction of residues. Techniques for the analysis of particulate matter include Fourier Transform infrared spectroscopy (FTIR), microcrystalline tests, polarized light microscopy, ion mobility spectroscopy (IMS), energy dispersive spectroscopy (EDS), X-ray powder diffraction (XRD), Raman spectroscopy, melting point tests, and flame tests. If particulate matter is absent, or if analyses of the particulates fail to identify the material, extractions of the debris are performed. The residues are extracted from the debris using both an organic and an aqueous wash to recover both organic and inorganic analytes. The organic extract might be analyzed by FTIR, IMS, HPLC/UV, HPLC/MS, gas chromatography with electron capture detection (GC-ECD), GC/MS, or MS/MS for confirmatory analysis of organic explosives. The aqueous extract might be analyzed by ion chromatography with UV detection (IC/UV), IC/MS, HPLC/MS, capillary electrophoresis with UV detection (CE/UV), elemental analysis, or X-ray analysis for confirmatory analysis of inorganic residues (5). 


\section{B. Overview of Explosives}

An explosion occurs when there is a sudden release of a large amount of energy. The source of the energy can be chemical, physical, or atomic in nature. The release of the energy can be in the form of pressure waves, propulsion of debris, or by the emission of ionizing and thermal radiation (6). Chemical explosions are the result of a chemical reaction or change in state which occurs over the course of a very short amount of time. A chemical explosive can be defined as a material, either a pure substance or a mixture of substances, which is capable of producing an explosion by release of its own energy (7). The result of a chemical explosion is the breakdown of explosives into more stable compounds, generally through the rapid conversion of liquids or solids into gases with a concomitant increase in pressure (8). This type of explosion is produced by materials which are compressed together, but not necessarily confined. During a chemical explosion, an extremely rapid (approximately 0.001 second) exothermic transformation occurs which results in the production of hot gases, vapors, and a pressure wave. Chemical explosives are primarily classified by their performance and uses, and have traditionally been divided into four classes: primary explosives, secondary explosives, blasting agents, and propellants. A subgroup class of explosives will also be discussed: improvised explosives.

Primary explosives, also referred to as primary high explosives, undergo a very rapid transition from burning to detonation, and can transmit the detonation to less sensitive explosives. Primary explosives have a high degree of sensitivity to initiation through friction, shock, heat, and electrostatic discharge. Upon detonation, the molecules in the primary explosive will dissociate and produce a tremendous amount of heat and 
pressure. This pressure wave can be used to detonate a second, more stable explosive. As a class, primary explosives differ in their sensitivity to heat and shock, as well as in their detonation velocities, which are in the range of 3500-5500 m/s. Examples of primary explosives include mercury fulminate, lead azide, and lead styphnate (๑).

Secondary high explosives are less sensitive to initiation than primary high explosives, and cannot be readily detonated by heat or friction. Secondary explosives can only be initiated to detonation by a powerful shockwave, such as that produced by detonation of a primary explosive. The detonation velocities of secondary explosives range from $5500-9000 \mathrm{~m} / \mathrm{s}$. Secondary explosives include the nitrate esters, nitrarenes, and nitramines. Nitroglycerin, nitrocellulose, and pentyerthritol nitrate (PETN) are nitrate esters; picric acid, 2,4,6-Trinitrotoluene (TNT), and tetryl are nitrarenes; RDX and HMX are nitramines $(6)(9)$.

Blasting agents are chemical mixtures consisting largely of ammonium nitrate. These explosives are relatively insensitive, and often require the use of a booster to achieve detonation. Blasting agents may take the form of slurries, emulsions, or gels. A binary explosive can be produced by mixing ammonium nitrate with fuel oil (ANFO), or ammonium nitrate with nitromethane (4). Binary explosives are the result of a mechanical blending of separate oxidizer and fuel components, as opposed to primary and secondary high explosives which contain the fuel and oxidizer within the same molecule. Though the components of a binary explosive may be relatively insensitive singularly, in combination they become a high explosive. The use of binary explosives is very common in commercial mining applications as well as in military applications. 
Propellants, also known as low explosives, are used commercially for firearms, rockets, and pyrotechnic displays. Examples of low explosives include black powder, black powder substitutes, pyrotechnic mixtures, smokeless powder, and flash powder (7) (8) (10) (11). Inorganic low explosives generally consist of a mixture of an oxidizer $\left(\mathrm{KClO}_{4}, \mathrm{KClO}_{3}\right.$, or $\left.\mathrm{KNO}_{3}\right)$ and a fuel (charcoal, sulfur, sugar, or metal powder). Low explosives undergo deflagration, which is the rapid combustion of materials at a rate less than the speed of sound through that material. Initiation of the reaction can be from a spark or hot wire. Deflagration creates high temperatures, but to create high pressure, the low explosives must be confined. This creates a pushing effect, whereas high explosives generate a shattering effect. Damage from a low explosive event is usually the result of fragmentation of the device and surrounding debris.

Improvised explosives are formulated from readily available ingredients. Similar to propellants and commercial binary explosives, improvised explosive mixtures combine an oxidizer with a fuel source. However, improvised mixtures are not commercially produced. Potential oxidizers include chlorates, perchlorates, hypochlorates, nitrates, chromates, dichromates, iodates, permanganates, and peroxides. Potential fuels include hydrocarbons, energetic hydrocarbons, and sulfur and metal fuels. The hydrocarbon category includes any material which can be burned to produce heat or energy. Examples are diesel fuel, kerosene, sugars, glycerin, wax, and sawdust. The energetic hydrocarbon category includes materials which contain oxygen, carbon, and hydrogen within the same molecule. For these materials, oxygen is commonly present in the form of nitro-groups. This means that energetic hydrocarbons such as nitrobenzene can make very powerful improvised explosives when mixed with an appropriate oxidizer. Nitromethane can even 
act as an explosive without the presence of a second oxidizer. The last fuel category includes phosphorus, sulfur, magnesium, titanium, and aluminum metals in powder form (12). The peroxide explosives triacetone triperoxide (TATP) and hexamethylene triperoxide diamine (HMTD) can also be classed with the improvised explosives, although they are not considered binary mixtures. As with nitromethane, the oxidizer and fuel components are contained within the same molecule. Depending on specific characteristics, improvised mixtures can act as low or high explosives, and as primary or secondary explosives.

\section{Featured Explosives}

\section{Black Powder Substitutes}

Black powder has been commercially available for several hundred years as a propellant for small arms fire. Traditional black powder is composed of $74 \% \mathrm{wt}$ potassium nitrate, $15.6 \% \mathrm{wt}$ charcoal, and $10.4 \%$ wt sulfur $(6)$. Black powder substitutes are alternatives to black powder, which have been formulated to have a more controlled burn rate, generate less smoke when fired, and improve the safety of storage (13). These propellants typically contain a carbonaceous organic fuel and inorganic oxidizers such as potassium nitrate $\left(\mathrm{KNO}_{3}\right)$ and/or potassium perchlorate $\left(\mathrm{KClO}_{4}\right)$. Depending on the brand of propellant, the fuel source may include charcoal, sulfur, sodium benzoate, dicyandiamide, nitrobenzoic acid, or ascorbic acid (14) (15). The presence of sulfur in conventional black powder causes residues such as sulfate and sulfide to form upon firing. These residues can corrode the barrel of a muzzle loading firearm over time. Therefore, many black powder substitutes currently on the market include a fuel that 
burns cleanly without the presence of sulfur. Ascorbic acid is a popular choice in part because use of this fuel results in a propellant which generates less smoke and residue upon firing. The water soluble nature of ascorbic acid residue can also improve clean-up between shots. These propellants are safer to handle than black powder, yet maintain comparable ballistic performance (16).

As manufacturers of ascorbic acid based black powder substitutes have merged, gone out of business, or sold their patents, the name and formulation of these propellants have changed. Ascorbic acid based black powder substitutes currently in production and available on the market in the USA include Jim Shockey's Gold, American Pioneer and Goex Pinnacle Replica. Older formulations which may not be currently in production or commercially available include Clean Shot, Clear Shot, Black Canyon, Golden Powder, and Black Mag3. Though this second set of powders may be more difficult to find, it is nonetheless important to recognize their existence as they may be available to people with criminal intent.

\section{Peroxide-based explosives}

Because of rising interest in homeland security and defense, improved technology has been developed for the detection of nitrated explosives. These improvements in detection capability of nitrated explosives have, in turn, led to an increased use of improvised explosive materials which do not contain nitro-groups, to avoid detection (17) or to circumvent barriers to the acquisition of commercial explosives. Peroxide explosives have drawn much attention over the past few years. As a currently emerging threat, peroxide-based explosives have been involved in several cases of terrorism (18) (19) (20) 
(21). Peroxide explosives may be solid, such as TATP or HMTD; or they may be liquid based, such as concentrated hydrogen peroxide/fuel mixtures. One advantage of peroxide based explosives is the relative ease with which the reagents or materials used in their

formulation can be procured. A variety of open-source literature detailing manufacturing methods for TATP and HMTD is readily available over the internet.

\section{a. Peroxide/fuel mixtures}

Contact between concentrated hydrogen peroxide and combustible materials such as wood, paper, and oil can cause immediate spontaneous combustion or ignition. An even more dangerous combination is the mixture of concentrated hydrogen peroxide with organic materials such as alcohols, aldehydes and their anhydrides, acetone, and ketones, because a spontaneous explosion can result. Contact between concentrated hydrogen peroxide and metals such as iron, copper, chromium, lead, silver, manganese, sodium, potassium, magnesium, nickel, gold, and platinum or metal alloys such as brass and bronze, can result in violent spontaneous explosions. Therefore, concentrated hydrogen peroxide is normally handled and stored inside of plastic containers. Spontaneous explosions can also result from unstable mixtures of concentrated hydrogen peroxide with concentrated mineral acids (22).

An example of the terrorist use of a liquid explosive made from concentrated hydrogen peroxide/fuel can be found in the failed July 21, 2005 attacks on the London transportation system. In this case, the main charge was a mixture of concentrated hydrogen peroxide mixed with white flour; initiation of the mixture occurred with a home-made TATP detonator (19). The foiled bombing of transatlantic flights in 2006 
also involved liquid explosives. This plot purportedly involved the use of concentrated hydrogen peroxide mixed with Tang (a powdered drink mix with a high sugar content) as the main charge (20).

\section{b. TATP and HMTD}

The explosive TATP can be prepared from the reaction of acetone in a sulfuric or hydrochloric acid solution with $45 \%$ hydrogen peroxide. For safety reasons, the reaction must be kept cold (23). Wolffenstein first described TATP in 1895 (24). The structure he proposed was confirmed in 1969 (25). The dimer of acetone peroxide is frequently observed as a byproduct of the synthesis. In some cases, tetramers of acetone peroxide may also be present (26). Triactone triperoxide is extremely dangerous to handle because of its instability. It is very sensitive to impact, friction, static electricity, and temperature changes, particularly when stored as a dry product. TATP also rapidly sublimates (27). The detonation velocity of TATP has been measured at nearly $5200 \mathrm{~m} / \mathrm{s}$, although there are indications that this value varies with diameter and density of the charge. The explosive performance of TATP has been calculated as approximately $62 \%$ that of TNT (28). The explosive HMTD can be prepared from hexamethylene tetramine and hydrogen peroxide in the presence of citric acid. Because it is exothermic, the reaction must be kept cold (23). The material was first synthesized by Legler in 1881 (29), and the structure was first proposed by Baeyer in 1900 (30). Like TATP, HMTD is extremely sensitive to friction and is very dangerous to handle.

The use of TATP and HMTD in improvised detonators has risen in recent years while the use of these explosives as the main charge in bombing incidents has also increased 
(12). On December 22, 2001, the "shoe-bomber" Richard Reid attempted to blow up American Airlines flight 63 with a device that included a TATP detonator and PETNbased main charge (18). On July 7, 2005, a series of four bombs containing organic peroxide charges were successfully detonated in the London transportation system by four suicide bombers. These attacks killed fifty-two people, and injured seven hundred more (21). These incidents demonstrate the pressing need to improve existing technologies and develop novel methods for both bulk and trace detection of peroxidebased explosives because of the increased incidence of their use and their lethality.

\section{Research Goals}

The overall goal of this research project was to expand the level of knowledge within the field of explosives analysis by developing better methods for the analysis of select explosives. The use of these explosives by terrorist groups or other criminal organizations requires the development of novel analytical techniques to determine what kind and how much explosive material was present. The aim of the first and second sections of this research project was the development of sensitive, rapid, and reliable methods for the analysis of both pre- and post-blast ascorbic-acid based propellants. The technique of ion chromatography with indirect photometric detection was chosen to utilize equipment which is currently available in most laboratories that perform explosives analysis. In contrast, quadrupole time-of-flight mass spectrometry was used in the second section of this research project, a technique currently beyond the capabilities of many forensic laboratories. However, the outstanding selectivity and degree-ofcertainty provided by the QToF instrument demonstrated in these experiments should 
make it an attractive choice for forensic laboratories to adopt in the near future. The aim of the third portion of this research project was to address the growing need for the development of a method for the analysis of concentrated hydrogen peroxide/fuel mixtures in both pre- and post-blast scenarios. The increasing use of these mixtures in explosives incidents indicate an urgent need for the development of improved analytical methods. 


\section{Chapter II. Instrumental Analysis of Explosives: Theory and Applications}

\section{A. Introduction}

Much work has been performed on the analysis of explosives by gas chromatography (GC). However, not all explosive materials are suitable for analysis by GC. For samples which lack thermal stability and/or volatility, high-performance liquid chromatography (HPLC) is an excellent alternative to gas chromatography. HPLC offers improved quantitative results and a higher sample capacity than GC. In addition, samples of lower concentration can be injected in higher volumes during HPLC analysis for improved sensitivity (31). With HPLC, ionic species can be analyzed easily, and derivatization of thermally labile materials is not required. Reversed phase HPLC and ion chromatography (IC) are efficient methods for analyzing explosives residues because minimal sample cleanup is needed, and detection systems are available which are both sensitive and selective (32) (33). Ion chromatography is frequently used in conjunction with capillary electrophoresis to minimize the problem of interfering ions, thereby allowing for clearer peak identification (34). The use of mass spectrometry provides both structural and molecular weight information. When employed in conjunction with a chromatographic technique, mass spectrometry can provide unequivocal identification of a compound of interest.

\section{B. Sample Collection and Preparation}

A general procedure for the analysis of explosives includes sampling, extraction, and instrumental analysis (35). A sample may be collected in its entirety, or the surface of a sample suspected to contain explosives residues may be swabbed for later laboratory 
analysis. The type of swabbing material may vary from one user to the next, and may be dependent upon the type of analysis which will be performed. For the collection of explosives traces from hands, Perret et al. (36) utilized cotton swabs while Zeichner et al. (37) utilized double sized adhesive stubs. For the collection of explosives traces from hard surfaces, Lahoda et al. (38), Popov et al. (39), and Thompson et al. (40) employed cotton swabs while Crowson et al. (41) utilized cotton/wool swabs. Following collection of explosives residues, the sampling media may be divided so that multiple extraction protocols can be performed to permit the recovery of both organic and inorganic explosives separately. For examples, in the work of Lahoda et al. (38), half of the cotton sampling medium was subjected to an aqueous extraction protocol to recover watersoluble explosives, while the other half of the cotton sampling medium was subjected to an organic extraction protocol to recover organic explosives.

Post-blast samples often include a variety of matrix components, thus, sample treatment is frequently required prior to instrumental analysis of the sample extract. Solid phase extraction (SPE) is a popular choice both for removing matrix materials from a sample and as a pre-concentration step. The choice of sorbent material in SPE is determined by the nature of the explosive material, and the nature of the matrix material (42). Ochsenbein et al. (43) successfully employed SPE cartridges with a polyvinylbenzene resin for the removal of matrix components and preconcentration of trace levels of RDX, HMX, PETN, and TNT from lake-water samples, while Tachon et al. (42) employed polymer sorbent materials for the preconcentration of organic explosives from mixtures with motor oil. 


\section{High Performance Liquid Chromatography}

High-performance liquid chromatography (HPLC) is a method of separating a sample into its various components. Separations are achieved by passing a sample dissolved in a solvent (the mobile phase) through a column packed with an adsorbent material (the stationary phase) under pressure. Components are separated based on their interactions with the stationary phase as they are swept through the column. There are a variety of different chromatographic modes available with HPLC, including partition chromatography, ion exchange chromatography, adsorption chromatography, and size exclusion chromatography. The analysis of explosives with HPLC is typically conducted by either partition chromatography or ion chromatography.

\section{Partition Chromatography}

Approximately $80-90 \%$ of HPLC separations are performed in partition chromatography mode (44). In normal phase HPLC, a polar stationary phase is combined with a non-polar mobile phase. Common stationary phase materials include bare silica- [Si-OH], cyanopropylsilyl- [-Si- $\left(\mathrm{CH}_{2}\right)_{3} \mathrm{CN}$ ], aminopropylsilyl [-Si- $\left.\left(\mathrm{CH}_{2}\right)_{3} \mathrm{NH}_{2}\right]$, and diol [Si- $\left.\left(\mathrm{CH}_{2}\right)_{3} \mathrm{OCH}_{2} \mathrm{CH}(\mathrm{OH}) \mathrm{CH}_{2} \mathrm{OH}\right]$ moieties. An example of normal silica can be seen in Figure 2.1. Hexane is a popular choice of mobile phase; additives such as medium chain length alcohols can be included in the mobile phase to make it more polar. In this mode of HPLC, the least polar compound elutes first. Normal phase chromatography is rarely, if ever, applied to the analysis of explosives.

Reversed phase HPLC combines a non-polar stationary phase with a polar mobile phase. Common stationary phase materials include n-octylsilyl- [-Si- $\left.\left(\mathrm{CH}_{2}\right)_{7} \mathrm{CH}_{3}\right]$ and n- 
octadecylsilyl- [-Si- $\left.\left(\mathrm{CH}_{2}\right)_{17} \mathrm{CH}_{3}\right]$ moieties. An example of $\mathrm{C}-18$ silica can be seen in Figure 2.1. Stationary phases that contain longer side chains are more retentive of nonpolar compounds. Water mixed with methanol, acetonitrile, and tetrahydrofuran are common choices for the mobile phase; increasing the percent of organic modifier will decrease the retention time of the analytes. In this mode of HPLC, the most polar compound elutes first. If there is no change in the composition of the mobile phase over the course of the analytical run, the separation is considered to be isocratic. In contrast, gradient separations involve a mobile phase composition that changes over time. For example, in a reversed phase gradient HPLC system, the separation might begin with $100 \%$ aqueous phase; climb to a 50\% aqueous/organic mobile phase; and finish with $100 \%$ organic phase. When using gradients, a re-equilibration period in the original mobile phase composition is generally required prior to beginning the next analytical run $(31)$.

\section{Ion-Exchange Chromatography}

Ion-exchange chromatography (IC) is a form of HPLC which is used to separate ionic analytes based upon the use of ion-exchange resins. This technique was first developed during the 1970s by Small et al. (45) where it was demonstrated that anion or cation mixtures could be readily resolved using HPLC columns packed with anionexchange or cation exchange resins. For this type of separation, the mobile phase is usually aqueous, although an organic portion of up to $10 \%$ organic phase may be utilized. The stationary phase of IC columns contains charge bearing functional groups attached to a polymer matrix with associated counter-ions to maintain electro-neutrality. For anion- 
exchange columns, chloride is commonly employed as a counter-ion, while for cationexchange columns, sodium is commonly employed as a counter-ion. In IC, the basis for separation lies in differences in the exchange equilibrium between sample ions and the eluent ion. Adjusting the $\mathrm{pH}$ of the mobile phase is one method of selectivity control (31) (46), as is adjusting the ionic strength.

Figure 2.1. Normal phase silica (left) and reversed phase C-18 silica (right): HPLC stationary phase material. Reversed phase HPLC is commonly employed in explosives analysis.
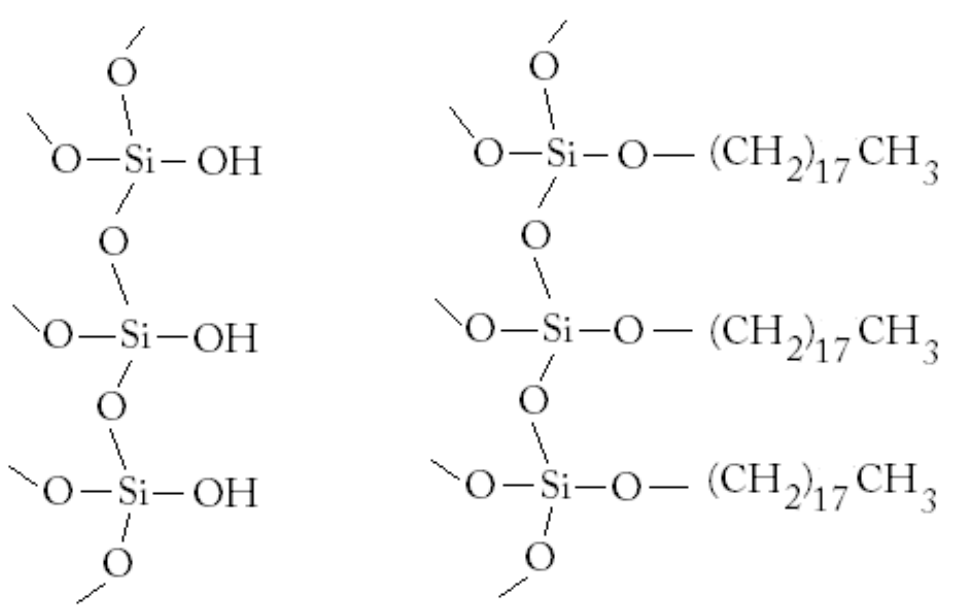

Ion-exchange equilibria between ions in solution and ions of like sign on the surface charge sites of the stationary phase is the process by which ion-exchange chromatography occurs. For anion exchange resins, the most common active sites include strongly basic tertiary amine groups -- $\mathrm{N}\left(\mathrm{CH}_{3}\right)_{3}{ }^{+} \mathrm{OH}^{-}$and weakly basic primary amine groups -- $\mathrm{NH}_{3}{ }^{+} \mathrm{OH}^{-}$. The most common active sites for cation exchange resins include the strongly acidic sulfonic acid group -- $\mathrm{SO}_{3}{ }^{-} \mathrm{H}^{+}$and the weakly acidic carboxylic acid group 
$-\mathrm{COO}^{-} \mathrm{H}^{+}$. When a strong base exchanger is brought into contact with an aqueous solution containing an anionic analyte, an exchange equilibrium is established.

Ion chromatography is an excellent technique for the detection of inorganic and organic ions. Depending on the detection method, this technique can be applied to the analysis of sub-parts per million levels of anions and cations. Because the inorganic ions present in explosive residues have a wide variety of sizes and charges, the two best chromatographic approaches are gradient elution with eluent conductivity suppression, or isocratic elution with a low capacity ion-exchange resin and indirect UV detection (11). Either technique can be used to analyze a full range of inorganic ions.

\section{Detection Systems for HPLC}

Detection systems for HPLC can be classified as either general or selective. A general detector will respond to most of the analytes which pass through the detection cell. Conductometric and mass spectrometric detectors are considered to be general detectors. In contrast, a selective detector will only respond to certain classes of analytes. Electrochemical, UV-VIS spectrophotometric, and fluorescence detectors are considered to be selective detectors. The primary benefit of using a general detector is the variety of analytes to which it can respond. This, however, can also be the primary disadvantage to using this type of detector. Selective detectors can offer the benefit of a lower limit of detection in comparison with general detectors because the lower background signals which result from selective detection translate into a reduced signal/noise ratio (46). When a high eluent background signal is present, or when a large amount of matrix 
materials are present, selective detectors can be very effective at picking out a small group of ions (or even a single ion).

\section{UV-VIS Absorbance Detection}

The most commonly employed detector for HPLC is UV-VIS absorbance detection (47). A typical UV detector contains a xenon lamp as a continuum UV source, and a photodiode array to detect transmitted light. The absorbance of analyte bands are measured as they pass through the detection cell. Use of a diode-array detector (DAD) allows for the collection of data at many different wavelengths during a single run. Spectra collected from a DAD can be used to confirm peak identity and test for peak purity (48).

The technique of HPLC-UV has been a popular choice for the analysis of explosives. Bender (49) analyzed the organic component of Pyrodex samples via HPLCUV with a C-18 column and a methanol/0.1N sulfuric acid (10:90) mobile phase with detection at $230 \mathrm{~nm}$. The EPA developed Method 8330 (50) for the analysis of fourteen organic explosives by HPLC-UV with C-18 and CN columns and a methanol/water (50:50) mobile phase with UV detection at $254 \mathrm{~nm}$. Guarav et al. (51) analyzed HMX and RDX from ground water and soil samples by HPLC-UV with a C-18 column and an acetonitrile/methanol /water (30:35:35) mobile phase with UV detection at 230nm. Wissinger and McCord (52) employed a gradient HPLC-UV procedure for the analysis of smokeless powders.

For analytes which do not absorb light in the UV range, indirect photometric detection (IPD) may be employed. This mode of detection permits the separation and 
detection of non UV-absorbing anions and cations without using a suppressor membrane (as in conductivity detection) (31). For indirect UV detection to be successful, the eluent anion must absorb in the visible or ultra-violet spectral region to establish a baseline level of absorbance. Elution of sample ions is monitored by measuring the decrease in absorbance at a specific detection wavelength as a fraction of the absorbing eluent ions are replaced by transparent sample ions (11) (46). For example when the solute anion (S-) is fully dissociated, the change in absorbance $(\Delta \mathrm{A})$ is measured by:

$$
\Delta \mathrm{A}=\left(\varepsilon_{\mathrm{S}-}-\varepsilon_{\mathrm{E}-}\right) C_{\mathrm{S}-}(b)
$$

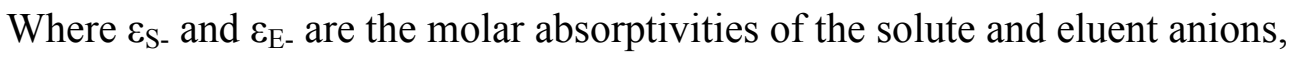

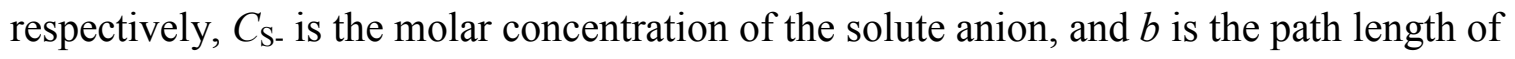
the cell. Generally, the detection wavelength and eluent anion are selected so the absorbance will decrease and solute peaks will be in the negative direction. However, the signal is usually electronically inverted (46).

For ion chromatography, it is important that the concentration of the elution ion $\left[\mathrm{E}^{-}\right]$be high enough to elute the sample ions within a reasonable period of time, and that it exceed the concentration of any ion in the sample $\left[\mathrm{S}^{-}\right]$. However, if $\left[\mathrm{E}^{-}\right]>>\left[\mathrm{S}^{-}\right]$then the background absorbance and noise of peak detection will be relatively high. A balance must be found between using a high eluent concentration to promote rapid ion exchange within the column, and using a low eluent concentration to reduce noise. It is best if the eluting ion selected for indirect photometric detection has a strong affinity for the ion exchanger so that a relatively low concentration can be used. In order to maximize sensitivity, the difference in molar absorptivities between the elution ion and sample ion should be as large as possible (31) (46). 
Verweij et al. (53) developed an IC-IPD method for the analysis of inorganic anions commonly found in low explosives: chloride, nitrate, nitrite, chlorate, sulfate, sulfide, carbonate, and perchlorate. This approach combined a weak anion exchange resin with an isopthalic acid mobile phase and indirect UV detection at $280 \mathrm{~nm}$. Bender (54) further developed this method for the analysis of thiocyanate in addition to the eight anions detected in the Verweij study. McCord et al. (34) employed the Bender version of this IC-IPD method in combination with a CE method for the analysis of inorganic residues on post-blast debris.

\section{Fluorescence Detection}

Fluorescence detection is based on the ability of certain molecules to emit light after excitation by ultraviolet radiation. During the excitation step, the absorption of a photon causes a solute molecule to move to an excited electronic state. In most cases, the absorbed energy will become dissipated through a series of vibrational transitions which result in the production of minute quantities of heat. This process will occur when the energy levels for the excited and ground states overlap. However, when there is no overlap between energy levels, the most convenient way for the excited molecule to lose energy is by emission of a photon. In this case, a fraction of the energy is dissipated without radiation until the lowest singlet energy level of the excited electronic state is reached. Between 1-100 ns later, the molecule falls back to one of the vibrational levels

of the ground state and emits a photon (see Figure 2.1) (44) (47). From this principle, it follows that the emitted light is always of a longer wavelength than the absorbed light. Because fluorescence is a rather rare phenomenon, detection based upon analyte 
fluorescence can be extremely selective, thereby making it ideal for trace analysis of complex samples. Fluorescence detection can be an order of magnitude more sensitive than UV detection, with a comparable linear range (55) as a result of reduced background signal.

Figure 2.2. Energy diagram of the fluorescence process. Fluorescence is a rare phenomenon, making it an extremely selective detection technique.

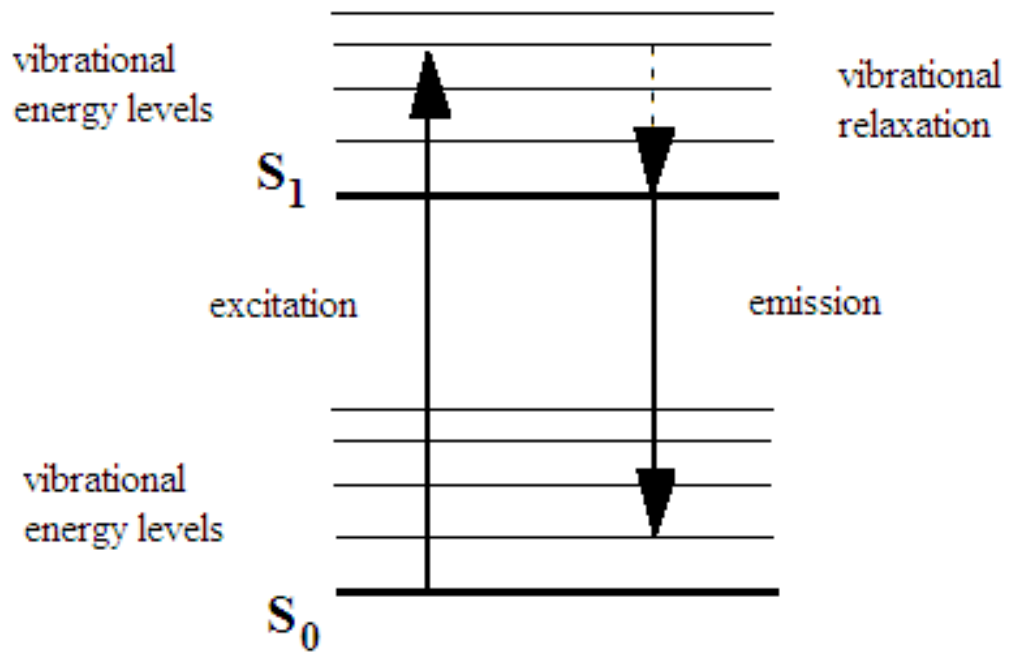

Compounds with fluorescent properties generally must be aromatic or contain pibonds. Because few analytes naturally possess fluorescent capabilities, two indirect methods of fluorescence detection may be employed. The first approach is similar to that of indirect photometric detection, in which analytes are detected based on a decrease in the fluorescent signal from a background electrolyte. This approach was employed by Goodpaster and McGuffin (56) in the analysis of seventeen nitrated explosives and their degradation products by capillary liquid chromatography with laser-induced fluorescence 
detection. Analytes were detected based upon their ability to quench a fluorescence signal emanating from a pyrene solution. The second approach is to derivatize the analyte via a reaction with a fluorophore-containing reagent. Derivatization can be performed either pre- or post-column, although post-column methods tend to be more reproducible and reliable. It is important the derivatizing reagent is not itself fluorescent, in order to minimize the background signal. It is also important that the derivatization reaction conditions are compatible with the HPLC separation conditions (47). Meng and Caddy (57) developed a reversed phase HPLC method which employed 9-

fluoroenylmethylchloroformate as a fluorogenic labeling reagent for the detection of ethyl centralite and 2,4-DNT in gunshot residues. Schulte-Ladbeck et al. (58) developed a reversed phase HPLC method with post-column UV irradiation and fluorescence detection for the analysis of TATP and HMTD. Following the separation step, HMTD and TATP were decomposed to hydrogen peroxide, which was subsequently detected via the peroxidase-catalyzed oxidation of $p$-hydroxyphenylacetic acid to yield the fluorescent dimer.

\section{Electrochemical Detection}

The term "electrochemical detection" is a broad term which encompasses several different techniques, including potentiometry, voltammetry, amperometry, and conductometry. Potentiometric detectors measure voltage, voltammetric and amperometric detectors measure current, and conductometric detectors measure resistance. In conductometry, the electrochemical (electrode) processes are secondary to the measurement of a bulk property of the solution. In conjunction with suppressor 
technology, conductometric detectors are frequently employed for the detection of ionic species. Hall and McCord (59), Doyle et al. (32), and Abramovich-Bar et al. (60) employed IC-CD for the analysis of inorganic ions, with an emphasis on the analysis of post-blast residues. Most recently, Johns et al. (3) employed ion chromatography with suppressed conductivity detection (IC-CD) for the analysis of eighteen anions and twelve cations commonly found in the post-blast residue of inorganic explosives. These methods were applied to the analysis of intact and post-blast commercial and improvised low explosive mixtures. Potentiometric detectors operate on the same principles as ionselective electrodes. In potentiometry, an indicating electrode measures a change in the applied potential in the presence of certain sample ions (46). However, potentiometry is not commonly used in HPLC, and has not been widely applied to the analysis of explosives. In voltammetry, a changing potential is applied to a working electrode, and the current resulting from the reduction or oxidation of an analyte at the electrode is measured. Square wave voltammetry has been applied to the analysis of 2,4-DNT and TNT in solution (61), and to the analysis of TNT, RDX, and HMX in soil samples (62). In amperometry, a fixed potential is applied to a working electrode, and the current resulting from oxidation or reduction reactions which occur at the electrode is measured. Amperometric detection has been applied to the analysis of explosives. Matysik et al. (63) analyzed fourteen nitroaromatic explosives by HPLC with simultaneous UV and electrochemical detection in oxidative mode. Marple and LaCourse (64) developed an HPLC-UV-ED method for the analysis of fourteen organic explosives in groundwater and soil samples. Separation of the explosives was performed on a C-18 column with a methanol/20 mM acetate mobile phase (50:50). Schulte-Ladbeck and Karst (65) 
developed an HPLC-ED method with post-column UV irradiation for the analysis of TATP and HMTD.

With amperometric detection, the potential required to induce electrolysis differs for each analyte. The useable potential range is typically limited to $-1.5 \mathrm{~V}-+1.5 \mathrm{~V}$. On the positive end, the working potential range is limited by the hydrolysis of water with the resulting liberation of hydrogen gas. On the negative end, the working potential range is limited by the reduction of dissolved oxygen in the eluent and in the sample. The reduction of water in the mobile phase to hydrogen gas is also a limiting factor at strongly negative potentials. Reduction/oxidation of mobile phase components increases the background signal by a considerable amount. Detector selectivity in amperometry is controlled by controlling the applied potential, the material of the working electrode, and the solution $\mathrm{pH}$. Because amperometry requires a conductive mobile phase in the establishment of a potential at the working electrode, it is used almost exclusively with reversed-phase HPLC. Amperometric detectors offer the benefits of a wide range of detector response (4-5 orders of magnitude), small cell dead volumes, ease of use, and low cost. Their primary disadvantage is that they can be subject to poisoning from contaminants. Also, detection is limited to electro-active materials. Amperometry is the most widely used method of electrochemical detection in HPLC (44) (46).

In amperometry, the working potential drives the electrochemical reaction at the surface of the working electrode. The potential applied to the working electrode is measured within the context of a known potential, which is in turn obtained from the reference electrode. The role of the reference electrode is to establish a stable potential. This electrode acts as a reference point along the potential axis by which the oxidizing 
power of the working electrode is measured. The potential axis is arbitrary and the reference electrode sets the zero point (66). The auxiliary electrode is used to prevent the electric current from passing through the reference electrode (because this could change the potential); instead, current passes between the working electrode and the auxiliary electrode. A potentiostat adjusts the auxiliary electrode potential to permit the measurement of current through the working electrode, while keeping the working electrode potential at the desired value relative to the reference (47).

When mobile phase flows through the electrochemical cell, a background current is generated due to oxidation or reduction of the eluent. When an electrochemically active analyte passes over the working electrode, it will be oxidized or reduced, and a change in the electrochemical current will be measured (67). The behavior of an amperometric cell can be described by Faraday's Law:

$$
Q=n F N
$$

The number of coulombs, $Q$, which are measured is related to the number of moles $N$ of electro-active species which are converted to a product by $n$ number of electrons per reaction ( $F$ is the Faraday constant). The rate of the electrochemical reaction is monitored by measuring the current through the working electrode. In the entire electrochemical process, three consecutive processes occur, the rates of which are proportional to the concentration of analyte in the solution. These include:

1. Transportation of the analyte molecules to the electrode surface by convective diffusion

2. Transfer of electrons between the analyte in solution and the electrode surface

3. Transport of reaction products away from the electrode surface 
Either the mass transport steps (\#1 and \#3) or the electron transfer step (\#2) can be ratelimiting. The rate of mass transfer is primarily determined by the geometry of the flow cell, while the rate of electron transfer is determined by the applied electrode potential (47).

Detection selectivity can be tuned by choosing a potential for the working electrode which is optimized for detection of the analyte of interest. Detector response for a given analyte is determined by the molecular structure of the analyte and its concentration, the applied potential in the detector cell, and the physical and chemical properties of the working electrode and chromatographic eluent (55).

A standard flow cell utilizes a three-electrode configuration (see Figure 2.3). The most common flow cell design for HPLC work is the thin-layer cell, in which the auxiliary electrode is positioned across from the working electrode to minimize the $i R$ drop between the two electrodes. In this type of cell, called an amperometric cell, only a few percent of the analyte in the eluent undergoes an electrochemical reaction. In contrast, coulometric detectors use a porous graphite working electrode that allows all of the mobile phase and analyte to be in contact with the electrode. Almost $100 \%$ of the analyte undergoes an electrochemical reaction in a coulometric detector (48) (68). Because of the large internal surface area of porous electrodes, the signal/noise ratios are inferior to those obtained with thin-layer cells. Nonetheless, coulometric detectors have been utilized in the detection of TATP and HMTD (65). 
Figure 2.3. Three electrode configuration for a standard amperometric flow cell used in electrochemical detection (67).

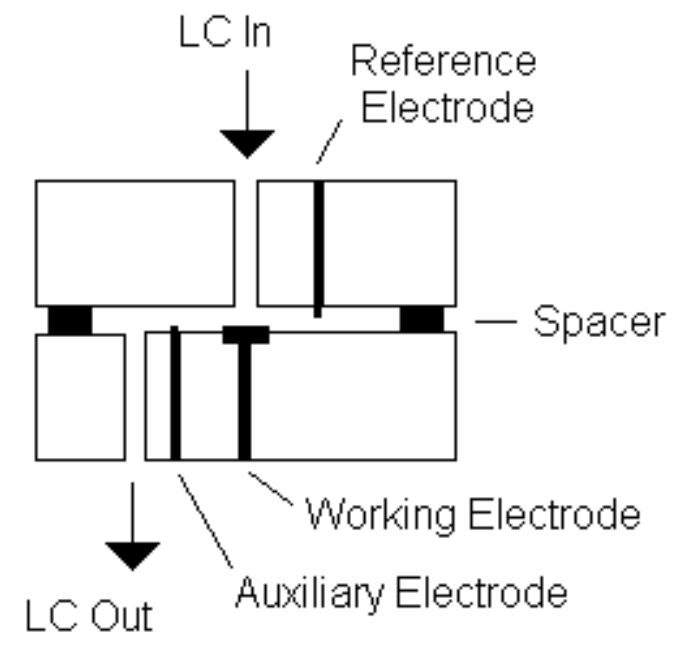

There are a variety of reference and working electrode combinations that may be employed in amperometric detection. One common choice for the reference electrode is the $\alpha$-hydrogen/palladium electrode. This electrode is maintenance free, stable, and not easily poisoned. It is important to note that there is a $300 \mathrm{mV}$ potential difference between the $\alpha$-hydrogen/palladium electrode and the silver/silver chloride electrode, which is another commonly employed reference electrode. If the applied potential is set to $+500 \mathrm{mV}$ relative to a silver/silver chloride reference electrode, then this is equivalent to an applied potential of $+200 \mathrm{mV}$ relative to a palladium/hydrogen reference electrode (66). Working electrodes can be made of noble metals such as gold, silver, or platinum, or they can be made of carbon, such as carbon paste, glassy carbon, and graphite. The potential range of each type of working electrode is different, so it is important to match the correct working electrode to the application. 
In direct current (DC) mode, a single potential is applied to the working electrode. Detection of analytes occurs at this set potential. In contrast, pulsed amperometric detection (PAD) cycles through a series of potential changes at a frequency of 0.5 to 3 Hz. Because only a single potential is applied in DC mode, oxidation products can gradually poison the surface of the electrode. Such poisoning of the electrode surface will cause a loss of analyte signal. The surface of the working electrode can be regenerated, but the flow cell must be dismantled so that mechanical polishing of the electrode can occur. In PAD mode, the electrode surface can be electrolytically cleaned by a series of potentials that are applied for fixed time periods after application of the detection potential in order to prevent signal loss. This series of potentials applied for a defined period of time is known as a waveform (67) (69). This mode of detection is particularly useful for applications where the working electrode is rapidly fouled due to adsorption of insoluble reaction products.

One cycle of PAD involves three steps: measurement, cleaning, and conditioning (see Figure 2.4). During the measurement step, the detection potential $E_{l}$ is applied for time interval $t_{1}$. Detection and data collection occur during time interval $t_{s}$ (sampling time). The time difference $t_{1}-t_{s}$ is the stabilization time. During the stabilization time, the charging current produced when changing potentials is allowed to decay, so that only current from analyte oxidation is measured during the detection period. During the cleaning step, potential $E_{2}$ is applied for time interval $t_{2}$. A monolayer of metal oxide forms at the surface of the working electrode due to the high positive potential. This potential must be high enough and long enough to completely oxidize the electrode surface. However, if the potential is too high, excessive metal oxidation will occur and 
the electrode will wear too rapidly (70). During the conditioning step, potential $E_{3}$ is applied for time interval $t_{3}$. The metal oxide which was formed during $t_{2}$ is electrochemically reduced by applying a negative potential, thereby renewing the electrode surface. Gold and platinum are the preferred materials for the working electrode in pulse mode because the cell current is stabilized more rapidly than in glassy carbon electrodes (67) (69). This mode of detection is sensitive to both flow rate and temperature. A wide range of applications have utilized PAD mode, including the analysis of sugars (69) (70) (71) (72), amino acids (73), tetracycline antibiotics (74), and histamine (75).

Figure 2.4. One cycle of pulsed amperometric detection (PAD). Sample components are detected at the working electrode at applied voltage $E_{l}$ during time period $t_{s}$. The working electrode is cleaned at applied voltage $E_{2}$ during time period $t_{2}$, and conditioned at applied voltage $E_{3}$ conditioned during time period $t_{3}$. This cycle of cleaning and conditioning steps regenerates the surface of the working electrode.

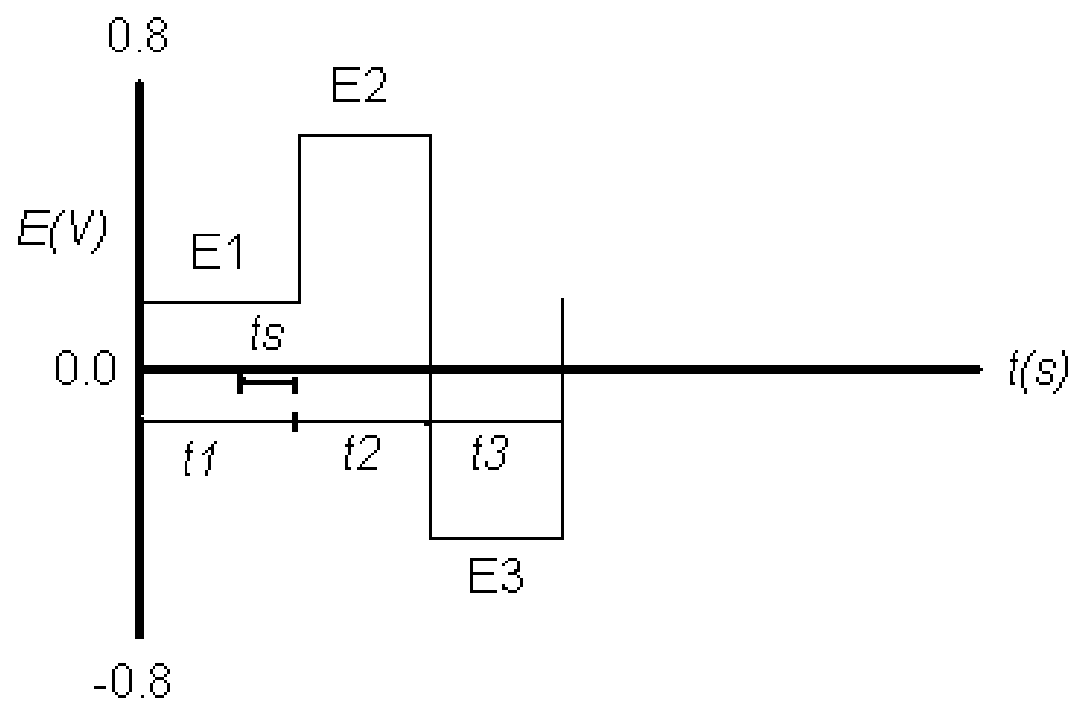




\section{Mass Spectrometry}

Detection by mass spectrometry allows for a more conclusive identification of unknowns than UV, fluorescence, conductivity, or electrochemical detection will permit. Mass spectrometers are designed to separate gas phase ions according to their mass to charge ratio $(\mathrm{m} / \mathrm{z})$. Commonly encountered mass spectrometers include quadrupoles, triple quadruples, ion traps, and time-of-flight instruments. The combination of two or more MS experiments is designated "MS/MS". The goal of an MS/MS experiment is to gain structural information by fragmenting ions isolated during the first MS experiment, and/or to achieve better selectivity and sensitivity for quantitative analysis. An MS/MS experiment can be performed by coupling multiple analyzers together (76).

The technique of HPLC-MS combines the separation power of HPLC with the detection power of mass spectrometry. Use of HPLC-MS allows for the separation of analytes and removal of interferences prior to introduction of the sample to the mass spectrometer. An interface between the HPLC system and the mass spectrometer is required to remove the HPLC solvent, and to generate the gas phase ions which will be transferred to the mass analyzer. Many modern mass spectrometric instruments utilize atmospheric pressure ionization (API) techniques, where solvent elimination and ionization steps are combined in the source and occur at atmospheric pressure. Common API techniques include atmospheric pressure chemical ionization (APCI), and electrospray ionization (ESI). In comparison to electron impact ionization (EI), ESI and APCI are considered to be soft ionization techniques which result in less fragmentation of the molecular ion in comparison to EI. The mass spectrum of an analyte ionized by ESI 
or APCI provides mainly molecular mass information, unless additional fragmentation techniques are applied.

Both ESI and APCI techniques have been applied to the analysis of explosives. Mathis and McCord (77) analyzed adducts of organic explosives by HPLC-ESI-MS. Xu et al. (78) employed HPLC-API-MS for the analysis of twenty-one nitroaromatic, nitramine, and nitrate ester explosives. Both APCI and ESI techniques were employed during this study. Xu et al. (79) analyzed TATP and HMTD via HPLC-APCI-MS/MS, where pseudomolecular ions were observed for these compounds. Dreifuss and Goodpaster (80) utilized HPLC-ESI-MS for the analysis of the organic and inorganic components of black powder substitutes.

Another popular method for the analysis of inorganic explosives is IC-MS. Norman and Ouderkirk (81) utilized ion chromatography with conductivity detection and mass spectrometric detection (IC-CD-MS) in series for the analysis of inorganic anions and cations in low explosives. Lang and Boyle (82) utilized IC-CD-MS for the simultaneous analysis of the organic and inorganic components of ascorbic acid-based low explosives.

The average mass of an analyte is calculated from the average atomic masses of its constituent elements. The nominal mass of an analyte is calculated from the nominal masses of the most abundant isotopes of its constituent elements. The exact (monoisotopic) mass of an analyte is calculated from the exact masses of the most abundant isotopes of its constituent elements. Mass resolution refers to the ability of an instrument to separate two adjacent masses; it measures the "sharpness" of the mass spectrometric peak. Mass accuracy refers to the accuracy of the mass information provided by the mass 
spectrometer, and can be expressed as the difference which is observed between the theoretical mass and the measured mass. This value is often expressed in parts per million (ppm) (see below).

$$
\begin{aligned}
& \Delta \text { mass accuracy }=\text { mass }_{\text {real }}-\text { mass }_{\text {measured }} \\
& \mathrm{ppm}=10^{6} * \Delta \text { mass accuracy } / \text { mass }_{\text {real }}
\end{aligned}
$$

Mass accuracy is linked to resolution; high mass accuracy cannot be provided by a low resolution instrument (because low resolution instruments are incapable of distinguishing between ions of less than 1 Da difference in mass). High resolution mass spectrometers include Time-of-Flight (ToF) and Quadrupole-Time-of-Flight (QToF) instruments; low resolution mass spectrometers include quadrupole, triple quadrupole, and ion trap instruments. Use of a high resolution instrument with an additional reference compound provides mass information with accuracy equal to or better than $\pm 5 \mathrm{ppm}$. For small molecules, this is sufficient to unambiguously determine the elemental composition of an analyte (76). The higher the instrument resolution is, the lower the mass error will be. To obtain potential formulae, the mass of the analyte must be measured with a high degree of accuracy, typically to four decimal places. To achieve this level of accuracy, a mass spectrometer needs to have high mass resolution to eliminate interference from ions of similar masses (83).

In time-of-flight mass spectrometry, ions formed in the source are extracted and accelerated to a high velocity by an electric field. This results in the application of the same kinetic energy to all of the ions. The ions then enter the mass analyzer, which consists of a long, straight drift tube. The ions pass through the drift tube until they reach a detector. After the initial acceleration, the velocity reached by an ion is inversely 
proportional to the square root of its $m / z$ value. Because the distance from the ion origin to the detector is fixed, the time taken for an ion to traverse the analyzer in a straight line is inversely proportional to its velocity, and directly proportional to the square root of its $\mathrm{m} / \mathrm{z}$ value. Therefore, each $\mathrm{m} / \mathrm{z}$ value has a characteristic time-of-flight from the source to the detector. Time-of-flight mass spectrometry measures each mono-isotopic mass based on the exact mass of the most abundant natural isotopes for that ion.

There are several equations which are relevant to time-of-flight mass spectrometry. The following abbreviations and constants hold: $\mathrm{E}=$ energy, $\mathrm{m}=$ mass, $\mathrm{z}=$ number of charges on an ion, $\mathrm{e}=$ charge on an electron, $\mathrm{v}=$ final velocity reached after acceleration. Following acceleration of an ion through an electric field, the kinetic energy of the ion is given by equation (1) which rearranges to form equation (2).

(1) $\mathrm{mv}^{2} / 2=\mathrm{z}^{*} \mathrm{e} * \mathrm{E}$
(2) $\mathrm{v}=(2 \mathrm{z} * \mathrm{e} * \mathrm{E} / \mathrm{m})^{1 / 2}$

If the distance from the ion source to the detector is given by $d$, then the time $(t)$ required for an ion to traverse the length of the drift tube is given by equation (3).

$$
\mathrm{t}=\mathrm{d} / \mathrm{v}=\mathrm{d} /\left(2 \mathrm{z}^{*} \mathrm{e}^{*} \mathrm{E} / \mathrm{m}\right)^{1 / 2}=\mathrm{d}^{*}\left[(m / z) /\left(2 \mathrm{e}^{*} \mathrm{E}\right)\right]^{1 / 2}
$$

In equation (3), $d$ is a fixed value, $E$ is held constant by the instrument, and $\mathrm{e}$ is a universal constant. Therefore, the flight time (t) of an ion is directly proportional to the square root of $m / z$ (equation 4)

$$
\mathrm{t}=(\mathrm{m} / z)^{1 / 2} * \text { a constant }
$$

In ToFMS, resolution is increased by bending the ion trajectory by an electronic mirror called a reflectron. Use of the reflectron increases the distance $\mathrm{d}$ from the source to the 
detector. When passing through the reflectron, dispersion of ions at the same $\mathrm{m} / \mathrm{z}$ is minimized, leading to a serious improvement in resolution.

In theory, there is no upper mass limit in ToFMS - all ions can be made to proceed from the source to the detector. In practice, however, there is a mass limitation because it becomes increasingly difficult to discriminate between arrival times at the detector as the $\mathrm{m} / \mathrm{z}$ values become quite large. Ionization techniques that produce multiply charged ions can extend the working range of the ToF analyzer, and the instrument offers the benefits of rapid cycling (83).

Figure 2.4 demonstrates a schematic of QToF instrument, a hybrid MS/MS system which couples a quadrupole mass filter [MS 1], a hexapole collision cell, and a ToF mass analyzer [MS 2]. The quadrupole mass analyzer employs four symmetrically arranged parallel rods. Rods which are diagonal to one another are connected together to a radio frequency $(\mathrm{RF})$ and direct current (DC) voltage generator. Ions which reach the quadrupole drift towards the detector. As they drift, they are influenced by the combined DC and oscillating RF fields. By ramping the alternating current (AC) and DC fields, ions of successive $m / z$ can pass through the quadrupole and impinge on the detector. The combination of these two mass analyzers allows for the performance of collision experiments, increased selectivity versus a single mass spectrometer instrument, high sensitivity in scan mode, a rapid scan rate, accurate mass measurements, and high resolution (76). 
Figure 2.5. Schematic of a hybrid quadrupole Time-of-Flight mass spectrometer (84).

This type of instrument can provide accurate mass measurement $\pm 5 \mathrm{ppm}$.

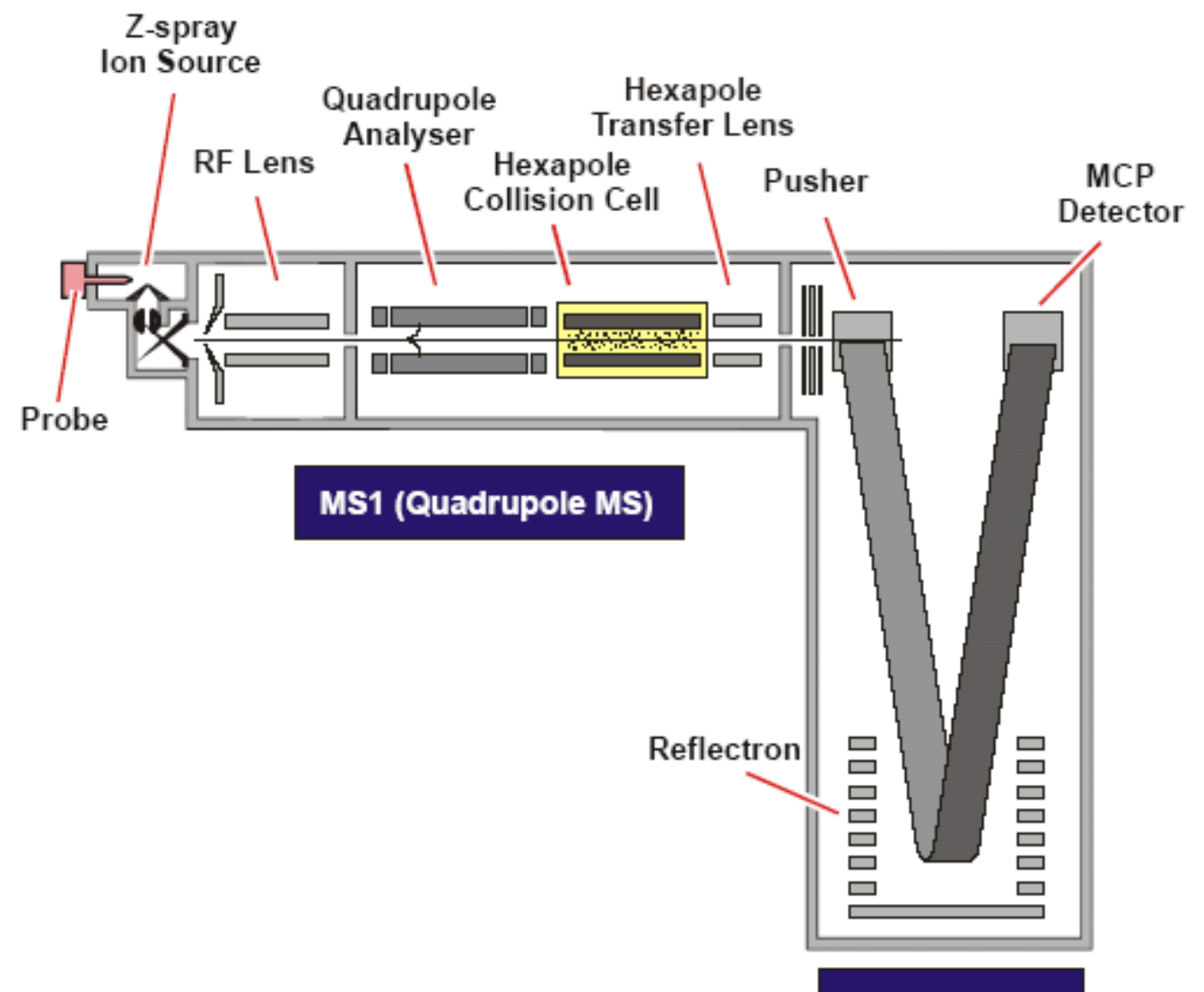

\section{MS2 (TOF MS)}

Conventional orthogonal acceleration time of flight (oa-ToF) mass analyzers similar to the Waters QToFMS Micro sample continuous beams of ions by asynchronously accelerating ions out of the acceleration region. A continuous beam of ions passes through the pusher region, out of which a fraction of the continuous beam is orthogonally accelerated. These ions are reflected by the reflectron towards the detector, as displayed in Figure 2.6a. In this asynchronous mode, the oa-ToF cannot analyze 100\% of the ions because a period of time between orthogonal pulses is required to prevent 
heavier ions from a preceding pulse from being overtaken by lighter ions from a subsequent pulse. The maximum duty cycle at a give $m / z$ is typically between $20-25 \%$, although this is dependent upon system geometry (85).

Figure 2.6. Schematic of an orthogonal analyzer-ToF (a) with asynchronous sampling of a continuous ion beam (85), and (b) with a T-Wave ${ }^{\mathrm{TM}}$ cell delivering packets of ions to the pusher (85).
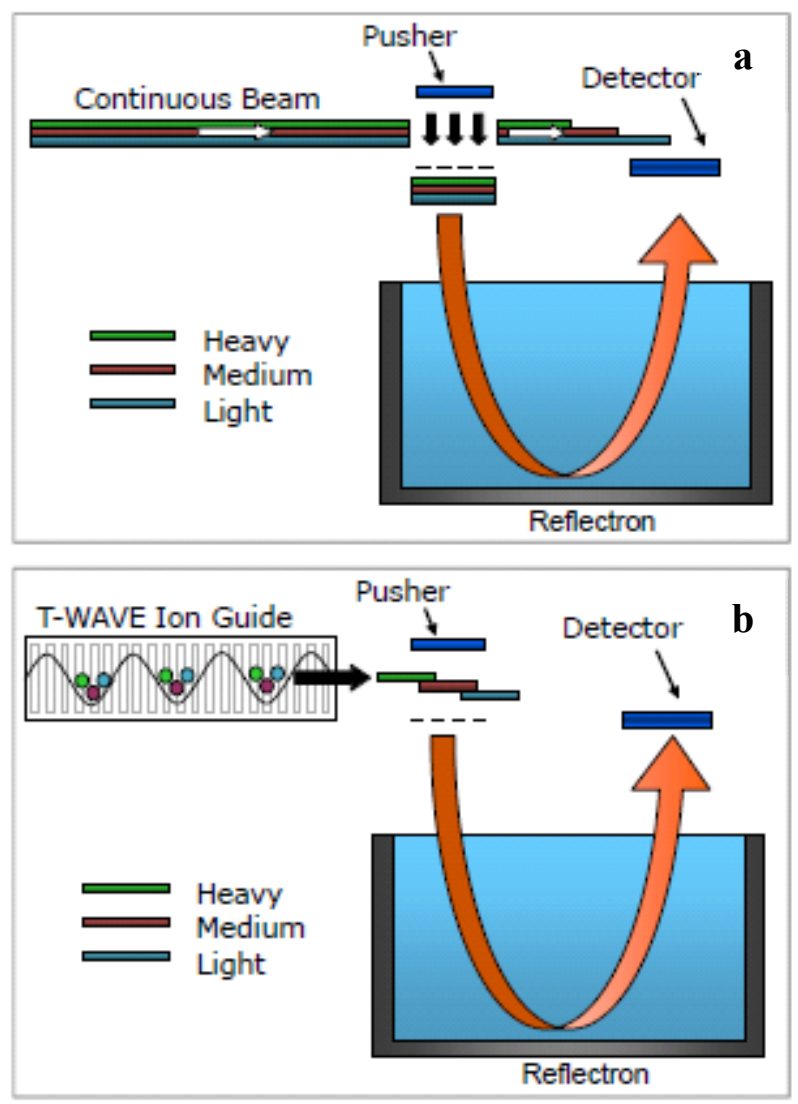
The duty cycle of a QToF can be enhanced over a restricted mass range by the addition of a traveling wave $\left(\mathrm{T}-\mathrm{Wave}^{\mathrm{TM}}\right)$ ion guide, as in the case of the Waters QToFMS Premier. The T-Wave ${ }^{\mathrm{TM}}$ ion guide is used to partition a continuous stream of ions into packets of ions of differing $\mathrm{m} / \mathrm{z}$ values. The ion packets travel through the collision cell where they are released at defined intervals. The activation of the oa-ToF pusher is synchronized for the arrival of the ion of interest into the extraction region, thereby maximizing the duty cycle for that particular ion. This is displayed in Figure 2.6b. This process is repeated, beginning with the timed release of the next packet of ions from the T-Wave ${ }^{\mathrm{TM}}$ cell.

Figure 2.7. Plot of the asynchronous duty cycle of an oa-ToF (black line) versus an enhanced duty cycle (red line). The enhanced duty cycle is obtained by synchronizing the pusher for $m / z 200 \mathrm{Da}(85)$.

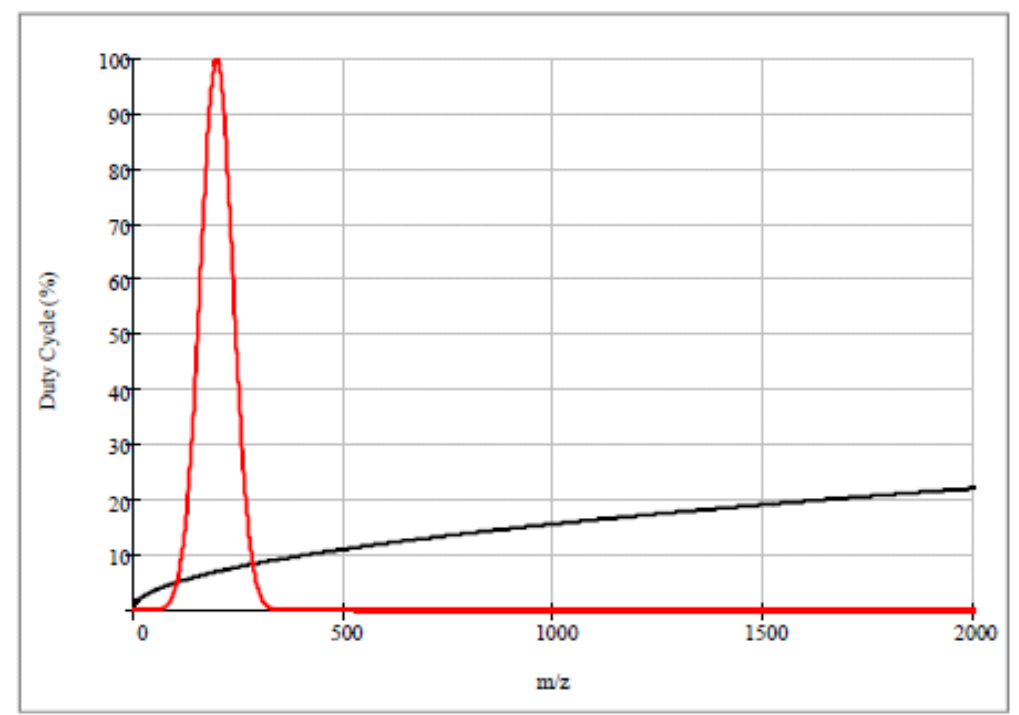


The increases in sensitivity offered by the T-Wave enabled enhanced duty cycle are dependent upon the $\mathrm{m} / \mathrm{z}$ of the target ion. For ions below $\mathrm{m} / \mathrm{z} 250$, an order of magnitude increase in sensitivity is possible, whereas at $\mathrm{m} / \mathrm{z} 800$ and above, increases of 3- to 4-fold are typical. Ions centered around the ion of interest which fall outside of a defined window are not pushed into the oa-ToF and are not acquired. Figure 2.7 is a plot of the asynchronous duty cycle of an oa-ToF compared to the enhanced duty cycle obtained by synchronizing the pusher with $\mathrm{m} / z 200 \mathrm{Da}(85)$ (86).

\section{E. Summary}

High performance liquid chromatography is an excellent technique for the separation of explosives and their residues. Depending upon the degree of certainty required for a given analysis, a variety of detector technologies are available. Indirect photometric detection, fluorescence detection, electrochemical detection, and quadrupole time-of-flight mass spectrometry are four very different types of detectors, yet each one is applicable to the analysis of explosives in conjunction with HPLC separations. 


\section{Chapter III. Experimental Procedures}

\section{A. Introduction}

The goal of this research was to develop chromatographic separation techniques for the analysis of novel explosives. Specifically, black powder substitutes and peroxidebased explosives were examined. The analysis of ascorbic acid-based black powder substitutes involved an analytical approach which utilized technology that was both low cost and widely available: ion chromatography with indirect photometric detection. This technique provided a rapid means to separate and detect the oxidizer and fuel components in ascorbic-acid based propellants. A second approach to the analysis of these low explosives involved the use of high performance liquid chromatography with electrospray ionization quadrupole time-of-flight mass spectrometry in negative ion mode. This method, while more time-consuming and complicated than the first approach, permitted the unequivocal identification of the oxidizer and fuel components in ascorbic acid-based propellants by combining both separation and spectrometric detection techniques. Liquid chromatographic techniques were also employed for the analysis of trace levels of hydrogen peroxide. Two complementary techniques were developed for this analysis: reversed-phase high performance liquid chromatography with post-column derivatization and fluorescence detection, and high performance liquid chromatography with electrochemical detection. The combination of these two methods for peroxide analysis provided excellent sensitivity, selectivity, and reproducibility. 


\section{B. Analysis of Ascorbic Acid-Based Propellants by IC-IPD}

\section{Chemicals}

Deionized (DI) water (18.3-megaohm) was obtained using a Millipore water purification system (Millipore, Bedford, MA). Reagent grade isophthalic acid (Aldrich Chemical Company, Milwaukee, MN, USA), potassium hydroxide (Spectrum Quality Products, Inc., New Brunswick, NJ, USA), and sodium hydroxide (Thermo Fisher Scientific, Pittsburgh, PA, USA) were acquired from chemical supply companies. Anion standards were prepared from reagent grade L-(+)-ascorbic acid (J.T. Baker, Phillipsburg, NJ), L-cysteine hydrochloride (Thermo Fisher Scientific), and potassium salts of chloride, chlorate, perchlorate, nitrite (Thermo Fisher Scientific), nitrate (Sigma-Aldrich, St. Louis, MO, USA), sulfate, cyanate, thiocyanate, and bicarbonate (Sigma-Aldrich). All electrolytes, standards, and extracts were prepared using 18.3 M $\Omega$ DI water. Anion standards were initially prepared at a concentration of $500 \mathrm{ppm}$ and diluted to a working concentration of $10 \mathrm{ppm}$.

The propellants used in this study included: Goex Black Rifle Powder grades FF and FFF (GOEX Inc., Doyline, LA, USA), Pyrodex grades RS and P (Hodgdon Powder Co, Inc., Mission, KS, USA), Triple 7 grade FFF (Hodgdon Powder Co, Inc., Mission, KS, USA), American Pioneer grades FF and FFF (American Pioneer Powder, Inc., Boca Raton, FL, USA), Jim Shockey's Gold grades FF and FFF (American Pioneer Powder, Inc., Boca Raton, FL, USA), Black Mag3 (Magkor Industries, Longwood, FL, USA), and Clean Shot (Clean Shot Technologies, Whitewater, CO, USA). 


\section{Instrumentation}

The ion chromatography system included an SSI 222D HPLC pump (Thermo Fisher Scientific) with an SP8880 autosampler (Thermo Fisher Scientific) and an ABI 783 programmable absorbance detector (Applied Biosystems, San Francisco, CA, USA). Separation was carried out using an IC-Pak Anion HR 4.6x75mm column (Waters Corporation, Milford, MA, USA) with a mobile phase of $4.5 \mathrm{mM}$ isophthalic acid at $\mathrm{pH}$ 4.2 with a flow rate of $1 \mathrm{~mL} / \mathrm{min}$ for $35 \mathrm{~min}$. A $20 \mu \mathrm{L}$ injection was used. Detection occurred at $280 \mathrm{~nm}$.

\section{Preparation of the Mobile Phase}

The anion eluent for IC contained $0.75 \mathrm{~g}$ isophthalic acid and $2 \mathrm{~mL}$ of $2 \mathrm{M}$ potassium hydroxide added to $3 \mathrm{~L}$ of boiling $18.3 \mathrm{M} \Omega$ DI water. The $\mathrm{pH}$ was adjusted to 4.2 with additional $2 \mathrm{M} \mathrm{KOH}$. The final electrolyte was stored in glass bottles at room temperature.

\section{Preparation of Samples}

\section{a. Powder Samples}

Twenty milligrams of unburned powder was used for each sample. To extract the ions, each powder was placed in a glass vial with $1.00 \mathrm{~mL}$ of $18.3 \mathrm{M} \Omega \mathrm{DI}$ water at room temperature and sonicated for twenty minutes. The extracts were filtered with $25 \mathrm{~mm}$ Acrodisc ${ }^{\circledR}$ syringe filters with a $0.45 \mu \mathrm{m}$ Supor ${ }^{\circledR}$ polyethersulfone (PES) membrane (Pall Life Sciences, Ann Arbor, MI). The filtered extracts were diluted prior to analysis by bringing $1.0-5.0 \mu \mathrm{L}$ of the original extract to a volume of $1.00 \mathrm{~mL}$ in $18.3 \mathrm{M} \Omega \mathrm{DI}$ 
water. Burned samples were prepared by placing 100 milligrams of each propellant on a clean, dry watch glass. A propane torch was used to deflagrate the sample on the watch glass. The burned residues were dissolved in $1.00 \mathrm{~mL}$ of $18.3 \mathrm{M} \Omega \mathrm{DI}$ water and allowed to sit for five minutes. The extracts were filtered with $25 \mathrm{~mm}$ Acrodisc $\AA$ syringe filters with a $0.45 \mu \mathrm{m}$ Supor ${ }^{\circledR}$ polyethersulfone (PES) membrane (Pall Life Sciences). Prior to analysis, the filtered extracts were diluted by bringing $1.0-5.0 \mu \mathrm{L}$ of the original extract to a volume of $1.00 \mathrm{~mL}$ in $18.3 \mathrm{M} \Omega \mathrm{DI}$ water.

\section{b. Preparation and Deflagration of Pipe Bomb Samples}

A pipe bomb study was conducted to examine differences in the quantity and type of anionic residues produced by various black powder substitutes. Additionally, a comparison was made between post-blast residues from the IEDs versus those obtained from open burning in the laboratory. The IED study utilized thirty-three polyvinyl chloride (PVC) pipes filled with explosive material that was deflagrated by the Miami Dade Police Department Bomb Squad. Each pipe bomb measured $2.54 \mathrm{~cm}$ in diameter x $12.7 \mathrm{~cm}$ in length, and included two end-caps. Individual pipes contained 14.2 grams of propellant. Seven different brands of black powder and black powder substitutes were utilized in the study. In addition, four of the propellants were obtained in two different grain sizes, resulting in a total of eleven different types of powder. Each powder was analyzed in triplicate for a total of thirty-three shots. Initiation of each explosive charge was performed via an electric match. Each individual pipe was suspended within its own plastic bucket to minimize contamination and maximize pipe fragment recovery. Cotton pads which had been previously cleaned with replicate washes of isopropanol and water 
were employed for residue collection. The interior of each bucket was dry swabbed as a control prior to deflagration. Post-blast residues were collected from inside the bucket with two additional dry swabs; pipe fragments which remained within the bucket were also collected. Each item was packaged separately to prevent cross-contamination. Postblast ions were extracted by placing $4 \mathrm{~cm}^{2}$ swatches of the post-blast swabs in glass vials with $1.00 \mathrm{~mL}$ of $18.3 \mathrm{M} \Omega \mathrm{DI}$ water for five minutes. The extracts were filtered with 25 mm Acrodisc ${ }^{\circledR}$ syringe filters with a $0.45 \mu \mathrm{m}$ Supor ${ }^{\circledR}$ polyethersulfone (PES) membrane (Pall Life Sciences). For each sample, $200 \mu \mathrm{L}$ of the filtered extract was diluted to 1.00 $\mathrm{mL}$ with 18.3 M $\Omega$ DI water prior to analysis.

\section{Analysis of Ascorbic Acid-Based Propellants by HPLC-ESI-QToFMS}

\section{Chemicals}

HPLC grade acetonitrile was used as received from Sigma Aldrich (SigmaAldrich, St. Louis, MO, USA). Deionized (DI) water (18.3-megaohm) was obtained using a Millipore Milli-Q water purification system (Millipore, Bedford, MA, USA). Reagent grade ammonium bicarbonate (Sigma-Aldrich), and ammonium hydroxide (Thermo Fisher Scientific, Pittsburgh, PA, USA) were acquired from chemical supply companies. Anion standards were prepared from reagent grade L-(+)-ascorbic acid (J.T. Baker, Phillipsburg, NJ, USA), and potassium salts of chloride, chlorate, perchlorate, nitrite (Thermo Fisher Scientific), nitrate, and sulfate (Sigma-Aldrich). All electrolytes, standards, and extracts were prepared using 18.3 M $\Omega$ DI water. Anion standards were initially prepared at a concentration of $1000 \mathrm{ppm}$ and diluted to a working concentration of 10 ppm. American Pioneer (American Pioneer Powder, Inc., Boca Raton, FL, USA), 
Jim Shockey's Gold (American Pioneer Powder, Inc), Black Mag3 (Magkor Industries, Longwood, FL, USA), Goex Pinnacle Replica (GOEX Inc, Doyline, LA, USA), were purchased during 2005-2007 from local sporting goods shops. Laboratory exemplars of 1994 Black Canyon (Legend Products, Las Vegas, NV, USA), and Clean Shot (Clean Shot Technologies, Whitewater, CO, USA) collected prior to 2005 were also analyzed.

\section{Instrumentation}

Two different instruments were used in this project, a Waters Quadrupole Timeof-Flight Premier mass spectrometer and a Quadrupole Time-of-Flight Micro mass spectrometer (Waters Corporation, Milford, MA, USA). Both instruments were coupled to a Waters UPLC system consisting of a high pressure pump, autosampler, solvent mixing system, and column oven. Because ultra-high pressure functionality was not required for this project, the Waters UPLC instrument was operated as an HPLC device. IC-Pak Anion HR 4.6 x $75 \mathrm{~mm}$ columns (Waters Corporation) were employed during the course of method development and sample analysis.

The aqueous component of the mobile phase (Solvent A) consisted of $10 \mathrm{mM}$ ammonium bicarbonate; ammonium hydroxide was used to adjust the $\mathrm{pH}$ to 10 . The organic component of the mobile phase (Solvent B) consisted of pure acetonitrile. The following mobile phase gradient profile was used: 0.0 minutes -4.0 minutes, $90 \%$ solvent $\mathrm{A} ; 4.0$ minutes -5.0 minutes, linear gradient to $50 \%$ solvent $\mathrm{A}$; hold $50 \%$ solvent A from 5.0 - 27.0 minutes; equilibrate back to $90 \%$ solvent A from 27.1-34.0 minutes. All separations were conducted at room temperature. A $5 \mu \mathrm{L}$ injection was used for all analyses, and the mobile phase flow rate was $0.5 \mathrm{~mL} / \mathrm{min}$. 
Electrospray ionization in negative mode was employed with both QToF instruments. The optimized parameters under which the mass spectrometers were operated are presented in Table 3.1. Because of differences in instrument design, several operating parameters were different for the two instruments. An important difference between the Premier and Micro instruments is that the Premier instrument permitted

synchronization of the operation of the T-wave ${ }^{\mathrm{TM}}$ collision cell and the TOF analyzer. This enhanced sensitivity over targeted $m / z$ ranges for detection of user-specified ions. This feature was employed during method development to target ascorbate, nitrate, and perchlorate to improve detection of these analytes at low concentrations. This feature was not available on the QToF Micro version of the mass spectrometer.

\section{Preparation of the Mobile Phase}

The aqueous portion of the mobile phase consisted of $10 \mathrm{mM}$ ammonium bicarbonate dissolved in $1 \mathrm{~L}$ of $18.3 \mathrm{M} \Omega \mathrm{DI}$ water. The $\mathrm{pH}$ of the mobile phase was adjusted to 10 though drop-wise addition of $29 \%$ ammonium hydroxide. The eluent was filtered through a $0.45 \mu \mathrm{m}$ nylon filter (Millipore) prior to use. The organic portion of the mobile phase consisted of HPLC grade acetonitrile. Both mobile phase components were stored in glass bottles at room temperature. 
Table 3.1. QToFMS parameters for the analysis of anion standards and propellant samples. Differences in design between the two QToFMS instruments required the use of different MS parameters.

\begin{tabular}{|c|c|c|}
\hline & QToFMS Premier & QToFMS Micro \\
\hline ESI Capillary Voltage & $8200 \mathrm{~V}$ & $3500 \mathrm{~V}$ \\
\hline Extraction Cone Voltage & $2.0 \mathrm{~V}$ & $0.0 \mathrm{~V}$ \\
\hline Sampling Cone Voltage & $12.0 \mathrm{~V}$ & $35.0 \mathrm{~V}$ \\
\hline Source Temperature & $120^{\circ} \mathrm{C}$ & $150^{\circ} \mathrm{C}$ \\
\hline Desolvation Temperature & $350^{\circ} \mathrm{C}$ & $350^{\circ} \mathrm{C}$ \\
\hline Desolvation Gas ( ${ }_{2}$ ) Flow Rate & $1150 \mathrm{~L} / \mathrm{hour}$ & $900 \mathrm{~L} / \mathrm{hour}$ \\
\hline Microchannel Plate Voltage & $2050 \mathrm{~V}$ & $2400 \mathrm{~V}$ \\
\hline Scan Range & $m / z 35-250$ & $m / z 45-500$ \\
\hline Scan Rate & $0.25 \mathrm{~s}$ & $1.0 \mathrm{~s}$ \\
\hline Resolution & 7908 & 5912 \\
\hline
\end{tabular}

\section{Instrument Calibration}

Because of significant variation in the air temperature of the room in which the QToFMS Micro was housed, the effective length of the flight tube $\left(L_{\text {teff }}\right)$ had to be checked on a daily basis. After corrections to $L_{\text {teff }}$ were made, the instrument was calibrated in negative ion mode at least once per day with a solution of sodium formate. To prepare the sodium formate calibration solution, $500 \mu \mathrm{L}$ of $0.10 \mathrm{M} \mathrm{NaOH}$ and $500 \mu \mathrm{L}$ of $10 \%$ formic acid in $18.3 \mathrm{M} \Omega$ DI water were diluted to a volume of $10.0 \mathrm{~mL}$ in $90 / 10$ isopropanol/water. This calibration solution was $0.050 \mathrm{M} \mathrm{NaOH}+0.50 \%$ formic acid in 
90/10 isopropanol-water. The calibration solution was stored in the refrigerator. The ESI interface has two electrospray tips that operate to alternately provide analyte ions (sample tip) or reference ions (LockSpray ${ }^{\mathrm{TM}}$ tip). During sample analysis, the same sodium formate reference solution which was used to calibrate the instrument was also used as the LockSpray ${ }^{\mathrm{TM}}$ solution. This solution was chosen as the reference solution because one of its cluster anions of sodium and formate at $\mathrm{m} / \mathrm{z} 180.9725$ was close to the exact mass of ascorbate (175.0243), and was towards the upper end of the range of masses which were being analyzed. When the sodium formate solution was used as the reference solution, a mass accuracy range of \pm 5 ppm or better for all target analytes was achieved. During sample analysis, sodium formate was pumped into the ESI source region at a rate of $5 \mu \mathrm{L} / \mathrm{s}$.

\section{Sample Preparation}

Standard solutions at $1000 \mathrm{ppm}$ of the individual anions of ascorbic acid, nitrate, nitrite, perchlorate, and chlorate were generated with 18.3 M $\Omega$ DI water. Extracts of intact explosives were prepared by dissolving ten grains of each propellant in $2.0 \mathrm{~mL}$ of 18.3 $\mathrm{M} \Omega \mathrm{DI}$ water, and sonicating for twenty minutes. Prior to analysis, the extracts were filtered using $0.2 \mu \mathrm{m}$ nylon syringe filters with $3 \mathrm{~mL}$ luer-lock plastic syringes (SigmaAldrich). Burned samples were prepared by placing 100 milligrams of each propellant on a clean, dry watch glass. A propane torch was used to deflagrate the sample on the watch glass. The burned residues were dissolved in $1.00 \mathrm{~mL}$ of $18.3 \mathrm{M} \Omega \mathrm{DI}$ water and allowed to sit on the watch glass for five minutes. The extracts were filtered using $0.2 \mu$ m nylon syringe filters with $3 \mathrm{~mL}$ luer-lock plastic syringes (Sigma-Aldrich) prior to analysis. 
A post-blast residue study was conducted to determine whether ascorbic acid and/or its degradation products in conjunction with the oxidizer anions could be detected using the gradient HPLC-ESI-QToFMS procedure. To obtain post-blast residues, six polyvinyl chloride (PVC) pipe bombs were constructed by employees of the ATF. Three of the devices were filled (2/3-full) with Jim Shockey's Gold, and three of the devices were filled (2/3-full) with Goex Pinnacle Replica. Each shot was initiated by a commercial electric match. PVC pipe fragments were collected and stored inside of metal containers. Post blast residues were recovered by extracting approximately 5 grams of PVC pipe fragments in $2.00 \mathrm{~mL}$ of $18.3 \mathrm{M} \Omega$ DI water and sonicating for twenty minutes. The extracts were filtered using $0.2 \mu \mathrm{m}$ nylon syringe filters with $3 \mathrm{~mL}$ luer-lock plastic syringes (Sigma-Aldrich) prior to analysis.

\section{Analysis of Hydrogen Peroxide by HPLC/ED and HPLC/FD}

\section{Chemicals}

HPLC grade water was used as received from Burdick \& Jackson (Honeywell Burdick \& Jackson, Morristown, NJ, USA). Deionized water (18.3-megaohm) was obtained using a Nanopure Infinity water purification system (Barnstead/Thermolyne, Pittsburgh, PA, USA). Reagent grade hemin powder (Sigma-Aldrich, St. Louis, MO, USA), 4-hydroxyphenylacetic acid (Acros Organics, Geel, Belgium), sodium acetate (Sigma-Aldrich), $10 \mathrm{~N}$ sodium hydroxide, sulfuric acid, EDTA (Thermo Fisher Scientific, Pittsburgh, PA, USA), ammonium hydroxide, ammonium chloride (SigmaAldrich), methanol (Honeywell Burdick \& Jackson), acetonitrile (Acros Organics), 50\% hydrogen peroxide in water (Sigma-Aldrich), and 30\% hydrogen peroxide (Riedel-de- 
Haen, Seelze, Germany) were purchased from chemical supply houses. Reagent grade benzoyl peroxide (Fluka, Pittsburgh, PA, USA), 70\% tert-butyl hydroperoxide in water, 98\% dicumyl peroxide, 97\% urea hydrogen peroxide (Sigma-Aldrich), 2-butanone peroxide (Fluka), and $80 \%$ cumene hydroperoxide (Sigma-Aldrich) were purchased from chemical supply houses.

\section{Instrumentation}

\section{a. High Performance Liquid Chromatography with Electrochemical Detection}

The HPLC/ED system consisted of a Waters Alliance 2695 Separations Module connected to a Waters 2465 Electrochemical Detector. The Alliance system included the following components: pump, autosampler, injector, solvent mixing system, and membrane degasser. Separation was carried out using CarboPac PA10 guard and analytical columns (Dionex Corporation, Sunnyvale, CA, USA) 4.6 x $75 \mathrm{~mm}$ and a mobile phase consisting of $150 \mathrm{mM} \mathrm{NaOAc}$ at $\mathrm{pH} 10.5$ at a flow rate of $1.00 \mathrm{~mL} / \mathrm{min}$. The injection size varied between $20 \mu \mathrm{L}$ and $100 \mu \mathrm{L}$. The guard and analytical columns were housed in the oven of the electrochemical detector. The columns and flow cell were maintained at $45^{\circ} \mathrm{C}$. A 3-mm gold working electrode was used in conjunction with a palladium-hydrogen reference electrode and a palladium working electrode. A 50 um spacer was used in the flow cell. Detection occurred in DC mode at an applied potential of $+400 \mathrm{mV}$. 


\section{b. High Performance Liquid Chromatography with Fluorescence Detection}

The HPLC/FD system consisted of a Waters Alliance 2695 Separations Module, two Waters 515 HPLC Pumps, and a Waters 474 Fluorescence Detector. The Alliance system included the following components: pump, autosampler, injector, solvent mixing system, and membrane degasser. Separation was carried out using an Acclaim 120 C-18 column (Dionex Corporation) which was 4.6 x $250 \mathrm{~mm}$. The mobile phase consisted of $1.00 \mathrm{mM} \mathrm{H}_{2} \mathrm{SO}_{4}$ and $0.10 \mathrm{mM}$ EDTA, which was delivered by the Waters 2695 Separations Module at a flow rate of $0.60 \mathrm{~mL} / \mathrm{min}$. Injection size varied between $10 \mu \mathrm{L}$ and $100 \mu \mathrm{L}$. The reagent solution contained $80 \mu \mathrm{M} p$-hydroxyphenylacetic acid and $8 \mu \mathrm{M}$ hemin dissolved in an ammonia buffer; this solution was delivered by the Waters 515 pump at a flow rate of $0.20 \mathrm{~mL} / \mathrm{min}$. The post-column reactor consisted of a length of Teflon tubing $0.5 \mathrm{~mm}$ ID x 2.0 meters long (Microsolv Technology, Eatontown, NJ, USA) A solution of $100 \mathrm{mM} \mathrm{NaOH}$ was delivered by the second Waters 515 HPLC pump at $0.20 \mathrm{~mL} / \mathrm{min}$. The Waters 474 Fluorescence Detector with grating technology included a standard flow cell of $100 \mu \mathrm{L}$; $\lambda_{\text {ex }}$ was $320 \mathrm{~nm}$ and $\lambda_{\text {em }}$ was $405 \mathrm{~nm}$.

\section{Preparation of Mobile Phases and Reagents}

\section{a. HPLC/ED}

The composition of the mobile phase was adapted from a Dionex technical note (70). A mobile phase that contained $150 \mathrm{mM} \mathrm{NaOAc}$ was prepared by dissolving 12.305 g NaOAc in 1.0 L of HPLC-grade water. The $\mathrm{pH}$ of this solution was initially 7.8 , then the $\mathrm{pH}$ was adjusted to 10.5 through drop-wise addition of $0.5 \mathrm{M} \mathrm{NaOH}$. The mobile 
phase was filtered through a $0.45 \mu \mathrm{M}$ nylon filter prior to use. The mobile phase was stored in glass bottles at room temperature.

\section{b. HPLC/FD}

The mobile phase was prepared by diluting $55 \mu \mathrm{L}$ of $96.1 \% \mathrm{H}_{2} \mathrm{SO}_{4}$ and $0.047 \mathrm{~g}$ EDTA in $1.0 \mathrm{~L}$ of HPLC grade water. The resulting solution contained $1.00 \mathrm{mM} \mathrm{H}_{2} \mathrm{SO}_{4}$ and $0.10 \mathrm{mM}$ EDTA. The mobile phase was filtered through a $0.45 \mu \mathrm{M}$ nylon filter prior to use. The base solution was generated by diluting $5.00 \mathrm{~mL}$ of $10.0 \mathrm{~N} \mathrm{NaOH}$ in $495 \mathrm{~mL}$

of 18.3 M $\Omega$ DI water to yield $0.50 \mathrm{~L}$ of $0.10 \mathrm{M} \mathrm{NaOH}$. Storage of both the mobile phase and base solution occurred in glass bottles at room temperature.

For the reagent solution, stock solutions of hemin were prepared by dissolving $13.0 \mathrm{mg}$ hemin in $10.0 \mathrm{~mL}$ of $0.10 \mathrm{M} \mathrm{NaOH}$. This solution was stored in a glass screwtop vial at $4^{\circ} \mathrm{C}$. Fresh stock solutions hemin were made fresh approximately once per month. Stock solutions of $p$-hydroxyphenylacetic acid were prepared by dissolving 30.4mg $p$-hydroxyphenylacetic acid in $10.0 \mathrm{~mL} 18.3 \mathrm{M} \Omega$ DI water. This second solution was stored in a glass screw top vial at room temperature. Fresh stock solutions of $p$ hydroxyphenylacetic acid were made fresh approximately once per month. The ammonia buffer was prepared by dissolving $2.68 \mathrm{~g} \mathrm{NH}_{4} \mathrm{Cl}$ and $25.0 \mathrm{~mL} 29 \% \mathrm{NH}_{4} \mathrm{OH}$ in $475 \mathrm{~mL}$ of 18.3 $\mathrm{M} \Omega \mathrm{DI}$ water to yield $0.50 \mathrm{~L}$ of solution. This third solution was stored at room temperature in a glass bottle. Fresh base solution was made as needed, typically about once per month. The reagent solution was prepared by diluting $0.20 \mathrm{~mL}$ stock solution of hemin and $0.15 \mathrm{~mL}$ stock solution of $p$-hydroxyphenylacetic acid to $50.0 \mathrm{~mL}$ with the 
ammonia buffer. The $\mathrm{pH}$ of this solution was 9.5. The reagent solution was filtered through a $0.2 \mu \mathrm{M}$ cellulose acetate filter prior to use. This solution was made fresh daily.

\section{Preparation of Hydrogen Peroxide Standards}

Standard solutions of hydrogen peroxide were generated weekly by diluting $50 \%$ reagent grade hydrogen peroxide in $18.3 \mathrm{M} \Omega$ DI water to yield a $5000 \mathrm{ppm}$ hydrogen peroxide standard. The $5000 \mathrm{ppm}$ hydrogen peroxide standard was diluted with $18.3 \mathrm{M} \Omega$ DI water to yield a $50 \mathrm{ppm}$ hydrogen peroxide standard. These standards were stored in the refrigerator at $4^{\circ} \mathrm{C}$. The $50 \mathrm{ppm}$ hydrogen peroxide standard was diluted with 18.3 $\mathrm{M} \Omega$ DI water to yield the daily working standard of $0.5 \mathrm{ppm}$ hydrogen peroxide.

\section{Preparation of Cotton Sampling Media}

Each cotton swab was subjected to the same cleaning procedure prior to use. Five swabs at a time were placed inside of a disposable $25.0 \mathrm{~mL}$ plastic luer-lock syringe (Sigma-Aldrich). The syringes were used to force $50.0 \mathrm{~mL} 18.3 \mathrm{M} \Omega \mathrm{DI}$ water through the swabs, followed by $50.0 \mathrm{~mL}$ isopropanol, followed by a second aliquot of $50.0 \mathrm{~mL} 18.3$ $\mathrm{M} \Omega$ DI water. The swabs were allowed to air dry at bench top conditions before they were packaged inside of a double layer of heat-sealed nylon bags. Analysis of water extracts from the cleaned swabs by HPLC/FD revealed no background signal, while analysis of water extracts from the cleaned swabs by HPLC/ED in PAD mode yielded a

small background peak which did not interfere with the detection of hydrogen peroxide. 


\section{Application and Recovery of Hydrogen Peroxide}

For the study regarding the effects of sampling on peroxide recovery, aliquots of $4.1 \mathrm{mg} \mathrm{H} \mathrm{O}_{2}$ were applied to the surface of paint chips and dried with a heat gun. Hydrogen peroxide residues were recovered either by performing a direct water rinse with $1.00 \mathrm{~mL}$ 18.3 M $\Omega$ DI water, swabbing with a cotton ball moistened with $0.50 \mathrm{~mL}$ 18.3 $\mathrm{M} \Omega \mathrm{DI}$ water followed by a water extraction in $2.50 \mathrm{~mL} 18.3 \mathrm{M} \Omega$ DI water, or dry swabbing followed by a water extraction of the cotton in $3.00 \mathrm{~mL}$ of $18.3 \mathrm{M} \Omega \mathrm{DI}$ water. Ten replicates were performed of each. The extracts were analyzed by HPLC/FD and HPLC/ED in DC mode. For this experiment, only cotton which had been cleaned according to the procedure outlined previously was used.

\section{Effect of Storage Conditions on the Recovery of Hydrogen Peroxide Residues}

A series of experiments were performed to explore the stability of hydrogen peroxide on a variety of porous and non-porous substrates under varying conditions for type of packaging material, storage temperature, and duration of storage. The substrates included denim fabric, concrete blocks, paint chips, and steel plates. The non-porous substrates were cleaned by washing with tap water, followed by a methanol rinse, followed by a rinse with $18.3 \mathrm{M} \Omega$ DI water. The denim was not cleaned or treated prior to use. An aliquot of $75.0 \mu \mathrm{L}$ of $5 \%$ hydrogen peroxide ( $4.1 \mathrm{mg}$ total) was applied to each substrate; the substrates were dried using the heat gun. The peroxide-treated substrates were stored either in paint cans or in a single layer of heat-sealed nylon bags. The packaged test materials were stored under one of two temperature/humidity conditions: room temperature $\left(T=23^{\circ} \mathrm{C}\right)$, and freezer $\left(T=-15^{\circ} \mathrm{C}\right)$. The materials were analyzed after 
one day, three days, and seven days of storage. Dry cotton swabs were utilized in the collection of hydrogen peroxide residues from all four substrates. Hydrogen peroxide residues were extracted from the swabs with 5.00 $\mathrm{mL} 18.3 \mathrm{M} \Omega$ DI water, and analyzed via HPLC/ED in DC mode.

\section{Survey of Law Enforcement Training Academy}

Thirty-four areas of a law enforcement training academy were sampled to determine the prevalence of hydrogen peroxide residues in an indoor setting. Each area was sampled with a cotton swab which was held with forceps. Each swab was stored in an individual screw-top glass vial. The swabs were extracted in 5.00 mL 18.3 M $\Omega$ DI water. The extracts were filtered through syringe filters prior to analysis by HPLC/FD and HPLC/ED.

\section{Collection and Analysis of Post-Blast Samples}

Experiments were conducted to test the optimized HPLC/ED and HPLC/FD methods, and to determine whether sufficient hydrogen peroxide survived detonation to be detected on post-blast debris. In the event that peroxide was recovered, the effectiveness of different types of substrates at retaining hydrogen peroxide residues was evaluated. Mixtures of concentrated hydrogen peroxide with fuel are extremely dangerous and prone to spontaneous detonation, and should only be handled by trained professionals wearing the proper safety gear. Personnel from the FBI Laboratory Explosives Unit prepared and detonated a small amount of three mixtures of $70 \%$ concentrated hydrogen peroxide and nitromethane under controlled conditions. The first 
two shots were detonated in September 2008, while the third shot was detonated in March 2009.

The first September 2008 shot was contained inside of a metal ammunition can which had been spray-painted neon pink to aid in the recovery of the fragments postblast. Three steel witness plates were set in a triangular pattern around the ammunition can at distances of 2.5 feet, 5 feet, and 10 feet. Each plate rested sixteen inches from the ground to the top of the plate. The ammunition can was covered by a white plastic cover to prevent overheating of the mixture prior to detonation. The second shot and larger shot from the September group was placed on the floor of the left rear passenger seat of an early model four door passenger car. A total of six steel witness plates were placed at varying heights and distances around the car. The shot from the March test was also contained inside of a metal ammunition can. While this can was not painted like the first can, it was covered with a plastic sheet prior to detonation. Three metal witness plates were set in a triangular pattern around the ammunition can at distances of 2.5 feet, 4 feet, and 5 feet; each plate rested approximately eighteen inches from the ground to the top of the plate. All three shots were initiated by an electric blasting cap. However, the March shot initially failed to detonate. A second blasting cap and a commercial Kinepak stick (a small amount of ammonium nitrate mixed with nitromethane) were used as a countercharge to detonate the unexploded mixture.

Following detonation of the first September shot, the witness plates were collected and packaged on-site inside of a double layer of heat-sealed nylon bags. Fragments from the ammunition can and the fiber cover were collected and packaged inside of a double layer of heat-sealed nylon bags. Soil samples from the blast crater were 
collected and stored inside of glass screw-top vials. All of these samples were stored in darkness at $-4^{\circ} \mathrm{C}$ until they were analyzed. The witness plates were analyzed the day after collection, one of the ammunition can fragments was analyzed one month after collection, and the rest of the post-blast debris was not analyzed until approximately five months after collection.

Following detonation of the second September shot, the witness plates were collected and packaged on-site inside of a double layer of heat-sealed nylon bags. Metal fragments from the device itself and from the car were collected and stored inside of metal paint cans. Fabric from the seat cover and roof lining of the car were collected. Some pieces were stored inside of metal paint cans while others were packaged in-site inside of a double layer of heat-sealed nylon bags. Clean, dry cotton swabs were used to sample different areas of the car, including the seat belts, floor boards, trunk, door handles, and side panels. The swabs were stored in glass screw top vials. All collected materials were stored at $-4^{\circ} \mathrm{C}$ for approximately five months until they were analyzed.

Following detonation of the March shot, the witness plates were collected and packaged on-site inside of a double layer of heat-sealed nylon bags. Fragments from the ammunition can and the fiber cover were collected and packaged inside of a double layer of heat-sealed nylon bags. These samples were stored at room temperature for two days until they were analyzed.

Hydrogen peroxide residues from all of the witness plates were extracted either by washing the front and back faces separately with $2.00 \mathrm{~mL} 18.3 \mathrm{M} \Omega$ DI water each, or by washing both faces with the same aliquot of $3.00 \mathrm{~mL} 18.3 \mathrm{M} \Omega \mathrm{DI}$ water. Residues from the cotton swabs were extracted in 4.00-6.00 mL 18.3 M $\Omega$ DI water, depending on the 
size of the swab. Residues from the ammunition can fragments were extracted either by directly rinsing the fragment with $2.00 \mathrm{~mL} 18.3 \mathrm{M} \Omega$ DI water, or by first using a dry cotton swab to collect the residues, and then extracting the cotton in $5.00 \mathrm{~mL} 18.3 \mathrm{M} \Omega \mathrm{DI}$ water. Residues were extracted from swatches cut from the plastic covers by rinsing the swatches in $2.00 \mathrm{~mL} 18.3 \mathrm{M} \Omega$ DI water. Soil samples were analyzed by sonicating $2.0 \mathrm{~g}$ of pulverized soil in $2.00 \mathrm{~mL}$ of $18.3 \mathrm{M} \Omega \mathrm{DI}$ water for twenty minutes. Residues from all other types of post-blast debris were collected by directly rinsing the debris with $2.00 \mathrm{~mL}$ 18.3 M $\Omega$ DI water. All samples were filtered with $0.45 \mu \mathrm{m}$ nylon filters using $3.00 \mathrm{~mL}$ luer lock plastic syringes. Samples were diluted as necessary prior to analysis. 


\section{Chapter IV. Analysis of ascorbic acid-based propellants by IC-IPD}

\section{A. Introduction}

Inorganic anions are both the products and reactants of the rapid combustion of oxidizers and fuels that occur during an explosion. Therefore, inorganic ion analysis is a routine part of an explosives residue detection protocol. It is of primary importance to be able to separate and detect these anions, as the nature of post-blast residue may be used to infer the original composition of the explosive (32). Ion chromatography is a powerful technique for the analysis of such materials, although it is not a confirmatory technique. Often, a second ion analysis technique with complementary separation selectivity to ion chromatography is employed for confirmation. One such example is capillary electrophoresis. While a given method of analysis may detect a variety of anions, some of the more commonly encountered anions in post-blast low explosives residue include chloride, nitrate, nitrite, perchlorate, and chlorate. Depending on the nature of the low explosive, additional anions of interest may include cyanate, thiocyanate, thiosulfate, phosphate, fluoride, acetate, benzoate, carbonate (87), and ascorbate.

\section{Previous research}

Bulk analytical techniques, such as X-ray powder diffraction (XRD), have been successfully applied to the identification of ascorbic acid in intact and well preserved powders (88). However, one can only distinguish between bulk propellants using such methods when the combined composition and morphology of the propellant granules are unique (80). Furthermore, in a post-blast scenario, unless a significant amount of un- 
reacted propellant is present, traditional bulk methods of analysis will fail to identify the original fuel material. In such cases, the use of other analytical techniques is required.

Over the past two decades, a great deal of research has been conducted on the separation and detection of the fuel and oxidizer components of black powder and black powder substitutes in both pre- and post-blast scenarios. Techniques which have been applied to the analysis of the fuel components of black powder substitutes include HPLCUV (49), GC-MS (89), ESI-MS (90), HPLC-MS and HPLC-MS/MS (15). Techniques which have been applied to the analysis of the oxidizer components of black powder substitutes include IC-IPD (34) (53) (54), IC-CD (3) (32) (60), CE-IPD (91) (92) (93) (94), and IC-MS (81). Approaches which combine the analyses of the oxidizer and fuel components of black powder substitutes include HPLC-MS (80), and IC-MS (82). Of these studies, only a few have focused on ascorbic-acid based black powder substitutes (80) (89) (82).

Verweij et al. (53) developed an IC-IPD method for the analysis of many inorganic anions of interest in the analysis of post-blast explosives residue, including chloride, nitrite, chlorate, nitrate, sulfate, sulfide, carbonate, and perchlorate. Bender (54) further refined this method for the analysis of these anions in pristine and burned samples of Goex Black Powder, Pyrodex, Golden Powder, and Solidox. Hargadon and McCord (93) developed a protocol which involved the concurrent use of an ion chromatography system with a separate capillary electrophoresis system for the determination of oxidizer anions present in the post-blast residue of black powder. The IC method was similar to that developed by Bender in 1989 (54): it utilized a weak anion exchange resin with a mobile phase which contained $4.5 \mathrm{mM}$ isophthalic acid at $\mathrm{pH} 4.6$; detection occurred at 
$280 \mathrm{~nm}$. Concurrent use of these two methods allowed for the separation and detection of chloride, nitrite, chlorate, nitrate, sulfate, thiocyanate, perchlorate, and chlorate. McCord et al. (34) later used these IC and CE methods concurrently for the analysis of the oxidizer anions present in a Pyrodex pipe bomb. Hopper et al. (91) also used this combination of CE and IC methods in the analysis of inorganic ions present in smokeless and muzzle-loading powders.

Other methods which have been developed for the analysis of inorganic anions and cations found in explosives residues include color tests (96), scanning electron microscopy-energy dispersion x-ray (96) (97), infrared spectroscopy (98), Raman spectroscopy (99), atomic absorption spectroscopy (100), portable capillary electrophoresis with indirect photometric detection (87), time-of-flight secondary ion mass spectrometry (101), and microchip electrophoresis with contact-less conductivity (102).

Outside of explosives analysis, the analysis of ascorbic acid and select inorganic anions is an issue which has also been heavily studied in the food science industry. One approach which permits the simultaneous analysis of organic acids and inorganic ions has been ion chromatography with conductivity detection (103) (104). Other studies have demonstrated the viability of analyzing organic acids and inorganic ions by capillary electrophoresis with both indirect and direct UV detection (105) (106) (107) (108). The method developed by Schiewe et al. was the first reported study to utilize cysteine as a stabilizer of ascorbic acid; a fifty-fold excess was used to prevent degradation of ascorbic $\operatorname{acid}(106)$. 


\section{Ascorbic acid-based propellants}

Black powder is composed of $74 \%$ wt potassium nitrate, $15.6 \% \mathrm{wt}$ charcoal, and $10.4 \%$ wt sulfur (6). It has been commercially available for several hundred years as a propellant for small arms fire. Black powder substitutes are alternatives to black powder which have been formulated to have a more controlled burn rate, generate less smoke when fired, and improve the safety of storage (13). Black powder substitutes typically contain a carbonaceous fuel and inorganic oxidizers such as potassium nitrate $\left(\mathrm{KNO}_{3}\right)$ and/or potassium perchlorate $\left(\mathrm{KClO}_{4}\right)$. A variety of fuel sources are available, including charcoal, sulfur, sodium benzoate, dicyandiamide, nitrobenzoic acid, or ascorbic acid (14) (15). The exact composition of black powder substitutes varies by manufacturer and brand.

The function of sulfur in traditional black powder is to improve ignition due to its lower melting temperature versus that of carbon. However, the presence of sulfur in traditional black powder can result in the formation of residues such as sulfate and sulfide upon firing. These residues can corrode the barrel of a muzzle loading firearm over time. Therefore, many black powder substitutes currently on the market include a fuel that burns cleanly without sulfur. Ascorbic acid is a popular choice in part because use of this fuel results in a propellant which generates less smoke and residue upon firing. The water soluble nature of ascorbic acid residue can also improve clean-up between shots. These propellants are safer to handle than black powder, yet maintain comparable ballistic performance (16). 
Pyrodex was the first black powder substitute to be introduced to the market in 1978. Pyrodex contained a fuel source which consisted of sodium benzoate and dicyandiamide in addition to sulfur and charcoal. Golden Powder was the first ascorbic acid based propellant to be introduced on the market in 1985. Golden Powder was followed by Black Mag and Black Canyon in 1996, Clean Shot in 1999, and Clear Shot in 2001. American Pioneer was introduced in 2003, followed by Goex Pinnacle Replica in 2004 and Jim Shockey's Gold in 2005. Black Mag was re-introduced as Black Mag3 in 2006 (82) (109). As the manufacturers of these ascorbic acid-based black powder substitutes have merged, gone out of business, or sold their patents, the name and formulation of these propellants have changed. The only ascorbic acid-based propellants currently in production and available on the market in the USA include Jim Shockey's Gold, American Pioneer, and Goex Pinnacle Replica.

The composition of seven of these propellants is outlined in Table 4.1. This table also includes a list of the anionic residues that result from deflagration of the propellant material. Anions which common to all seven brands of propellant include nitrate, nitrite, cyanate, and carbonate. Upon deflagration, Goex Black Powder and Pyrodex also produce sulfate, thiocyanate, and hydrogen sulfide; while Triple 7, American Pioneer, Black Mag3, Clean Shot, and Jim Shockey's Gold also produce perchlorate, chlorate, and chloride. Because the latter group of propellants yield the same types of residues upon deflagration, it can be difficult (if not impossible) to distinguish one brand from another based on the presence or absence of anionic residues. 
Table 4.1. Composition of propellants utilized in this study and their expected post-blast inorganic anionic residues (34) (89). Anions common to all seven brands of propellant include nitrate, nitrite, cyanate, and carbonate. Goex Black Powder and Pyrodex also produce sulfate, thiocyanate, and hydrogen sulfide; while Triple 7, American Pioneer, Black Mag3, Clean Shot, and Jim Shockey's Gold also produce perchlorate, chlorate, and chloride.

\begin{tabular}{|c|c|c|}
\hline Propellant & Components & Anions \\
\hline Goex Black Powder & $\mathrm{KNO}_{3}, \mathrm{~S}, \mathrm{C}$ & $\begin{array}{c}\mathrm{NO}_{3}^{-}, \mathrm{NO}_{2}^{-}, \mathrm{HS}^{-}, \mathrm{SO}_{4}^{2-} \\
\mathrm{OCN}^{-}, \mathrm{SCN}^{-}, \mathrm{HCO}_{3}^{-}\end{array}$ \\
\hline Pyrodex & $\begin{array}{c}\mathrm{KNO}_{3}, \mathrm{~S}, \mathrm{C}, \mathrm{KClO}_{4}, \\
\mathrm{NaOBz} \text {, Dicyandiamide }\end{array}$ & $\begin{array}{c}\mathrm{NO}_{3}^{-}, \mathrm{NO}_{2}^{-}, \mathrm{HS}^{-}, \mathrm{SO}_{4}^{2-} \\
\mathrm{OCN}^{-}, \mathrm{SCN}^{-}, \mathrm{HCO}_{3}^{-}, \mathrm{Cl}^{-} \\
\mathrm{ClO}_{4}^{-}, \mathrm{ClO}_{3}^{-}\end{array}$ \\
\hline Triple 7 & $\begin{array}{c}\mathrm{KClO}_{4}, \mathrm{KNO}_{3}, \mathrm{C} \\
\mathrm{NaOBz} \text {, Dicyandiamide }\end{array}$ & $\begin{array}{c}\mathrm{NO}_{3}^{-}, \mathrm{NO}_{2}^{-}, \mathrm{OCN}^{-}, \mathrm{HCO}_{3}^{-}, \\
\mathrm{Cl}^{-}, \mathrm{ClO}_{4}^{-}, \mathrm{ClO}_{3}^{-}\end{array}$ \\
\hline American Pioneer & $\mathrm{KClO}_{4}, \mathrm{KNO}_{3}, \mathrm{AA}$ & $\begin{array}{c}\mathrm{NO}_{3}^{-}, \mathrm{NO}_{2}^{-}, \mathrm{OCN}^{-}, \mathrm{HCO}_{3}^{-}, \\
\mathrm{Cl}^{-}, \mathrm{ClO}_{4}^{-}, \mathrm{ClO}_{3}^{-}\end{array}$ \\
\hline Jim Shockey's Gold & $\mathrm{KClO}_{4}, \mathrm{KNO}_{3}, \mathrm{AA}$ & $\begin{array}{c}\mathrm{NO}_{3}^{-}, \mathrm{NO}_{2}^{-}, \mathrm{OCN}^{-}, \mathrm{HCO}_{3}^{-}, \\
\mathrm{Cl}^{-}, \mathrm{ClO}_{4}^{-}, \mathrm{ClO}_{3}^{-}\end{array}$ \\
\hline Black Mag & $\mathrm{KNO}_{3}, \mathrm{KClO}_{4}, \mathrm{AA}$ & $\begin{array}{c}\mathrm{NO}_{3}^{-}, \mathrm{NO}_{2}^{-}, \mathrm{OCN}^{-}, \mathrm{HCO}_{3}^{-}, \\
\mathrm{Cl}^{-}, \mathrm{ClO}_{4}^{-}, \mathrm{ClO}_{3}^{-}\end{array}$ \\
\hline Clean Shot & $\mathrm{KNO}_{3}, \mathrm{KClO}_{4}, \mathrm{AA}$ & $\begin{array}{c}\mathrm{NO}_{3}^{-}, \mathrm{NO}_{2}^{-}, \mathrm{OCN}^{-}, \mathrm{HCO}_{3}^{-}, \\
\mathrm{Cl}^{-}, \mathrm{ClO}_{4}^{-}, \mathrm{ClO}_{3}^{-}\end{array}$ \\
\hline
\end{tabular}




\section{Properties of ascorbic acid}

Ascorbic acid is more commonly known as vitamin C. As seen in Figure 4.1, ascorbic acid has a pKa $\mathrm{pa}_{1}$ of 4 and a $\mathrm{pKa}_{2}$ of 11.8 ; the deprotonated form of ascorbic acid is referred to as ascorbate. This form of ascorbic acid is readily detected at $265 \mathrm{~nm}$. One factor which can complicate the detection of ascorbic acid is that upon exposure to an oxidizing environment, ascorbic acid begins to degrade rapidly, particularly when in the presence of heat, light, moisture, or metal ions (110). Depending upon the conditions, ascorbic acid can form a variety of degradation products (111) (112). The initial degradation pathway has been previously established (89) (113) (114): ascorbic acid first oxidizes reversibly to dehydroascorbic acid, which then degrades irreversibly to 2,3diketogulonic acid. Goodpaster and Keto (89) illustrated the reaction pathway listed in Figure 4.2, whereby the 2,3-diketogulonic acid further degrades to form threonic acid and oxalic acid.

Figure 4.1. $\mathrm{Pk}_{\mathrm{a}}$ of ascorbic acid. Ascorbate is the singly charged form of ascorbic acid.<smiles>O=C1OC(C(O)CO)C(O)=C1O</smiles><smiles>O=C1OC(C(O)CO)C([O-])=C1O</smiles><smiles>O=C1OC(C(O)CO)C([O-])=C1[O-]</smiles>

A more detailed degradation pathway for ascorbic acid was offered by Cioffi et al. (115). In this pathway, which is presented in Figure 4.3, dehydroascorbic acid rapidly undergoes re-hydration, followed by spontaneous hydrolysis at the lactonic ring. This 
results in the formation of the diketogulonate anion in its monohydrated and/or dehydrated forms.

Figure 4.2. Degradation process of ascorbic acid resulting in the formation of 2,3diketogulonic acid, oxalic acid, and threonic acid (89). Ascorbic acid will irreversibly degrade in the presence of heat, light, water, and metal ions.

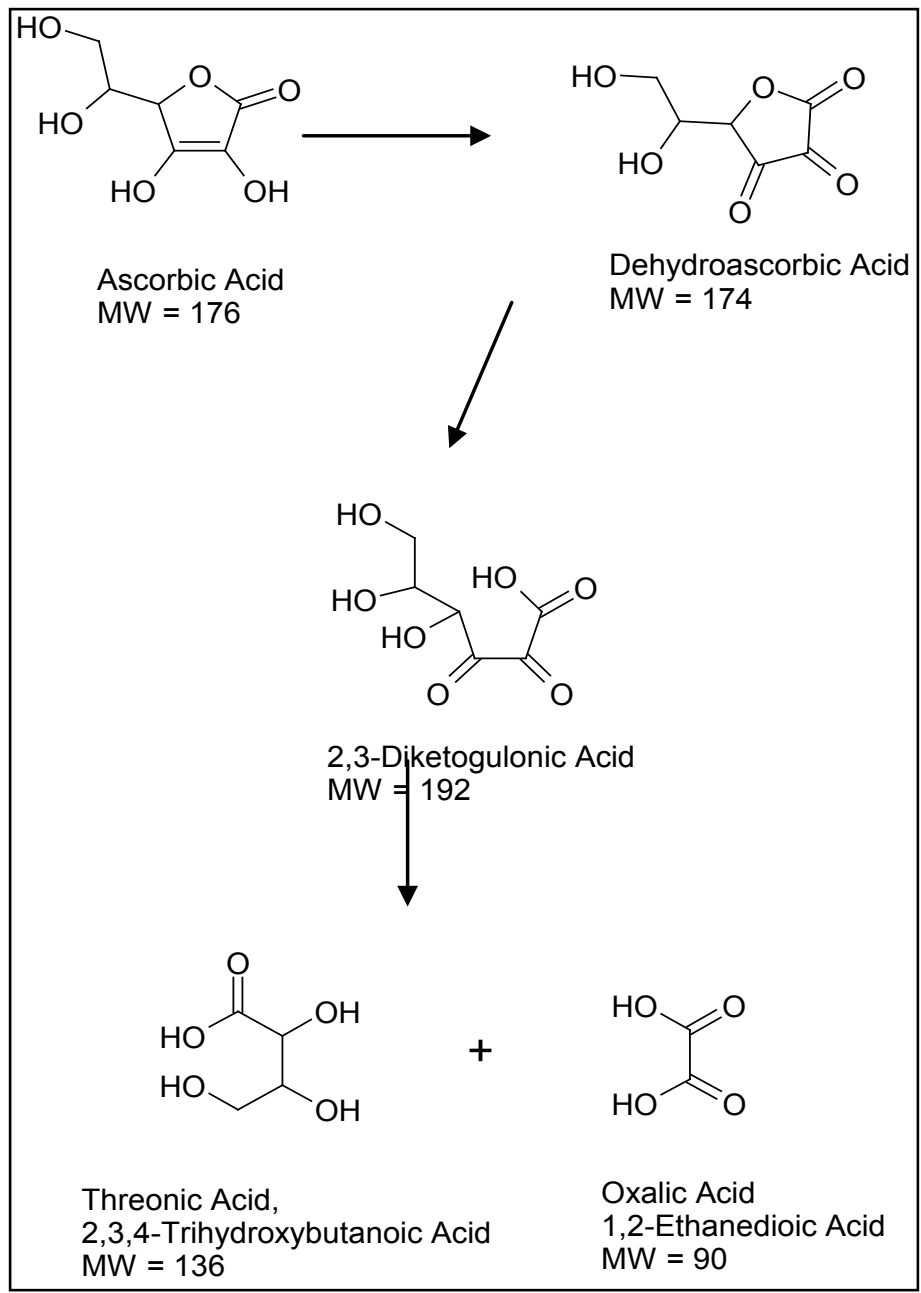


Figure 4.3. Pathway for the formation of diketogulonic acid from ascorbic acid (115).

Further degradation of diketogulonic acid will yield oxalic acid and threonic acid.<smiles>O=C1OC(C(O)CO)C(=O)C1=O</smiles>

Dehydroascorbic Acid (DAA) $\mathrm{MW}=174$
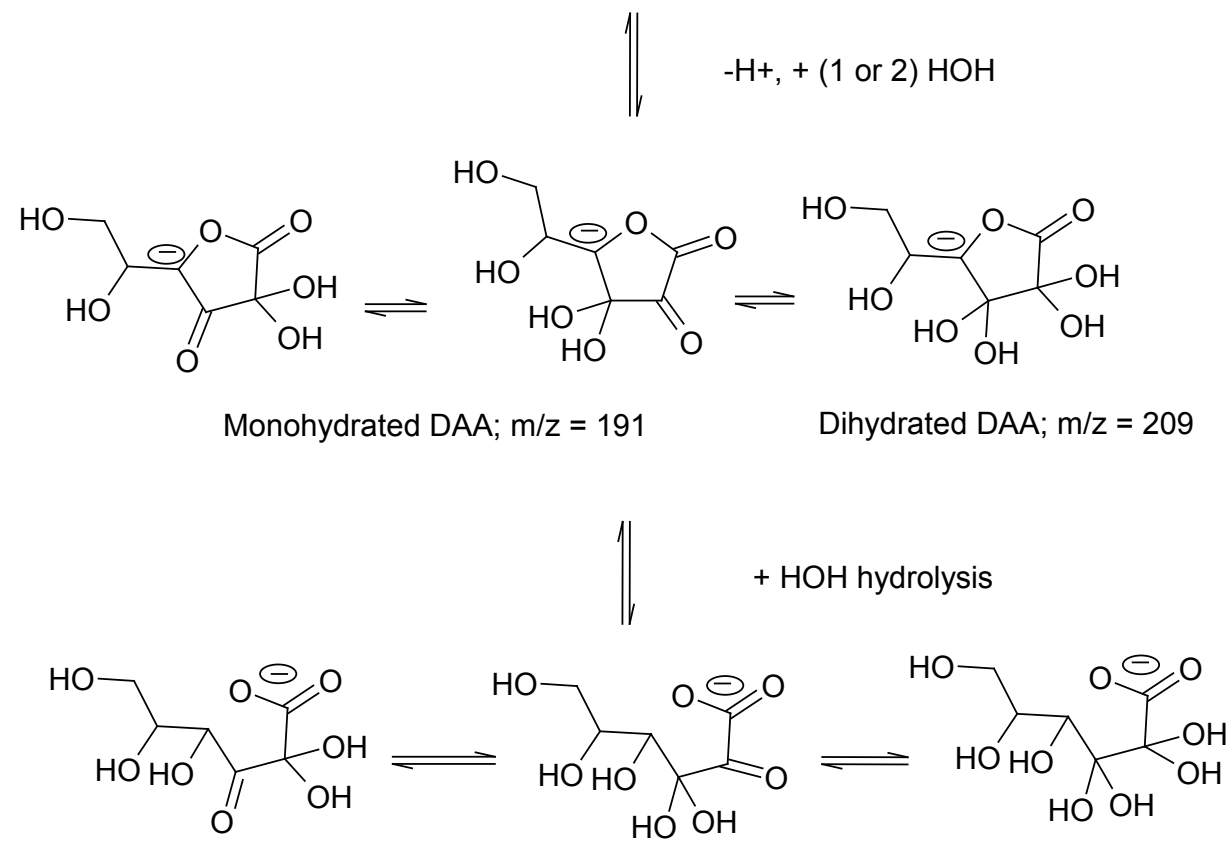

Monohydrated diketogulonate, $\mathrm{m} / \mathrm{z}=209$

Dihydrated diketogulonate, $\mathrm{m} / \mathrm{z}=227$

Research within the food science industry has shown that the addition of a competing reducing agent with a more negative redox potential (such as L-cysteine) will slow the degradation of ascorbic acid, thereby increasing the window in which an aged sample can be analyzed (106). An excess of cysteine can be added as needed to enhance the stability of ascorbic acid. 
Figure 4.4. Oxidation of ascorbic acid to dehydroascorbic acid (top) and oxidation of cysteine to cystine (bottom). The addition of cysteine to ascorbic acid samples can reduce the rate of ascorbic acid degradation.<smiles>NC(CS)C(=O)O</smiles>

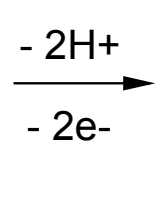<smiles>O=C1OC(C(O)CO)C(=O)C1=O</smiles>

\section{B. Development of IC-IPD Method}

Previous studies (34) (53) (54) determined that an isophthalic acid mobile phase coupled with an anion exchange resin was a useful procedure for the detection of inorganic anions present in low explosives. As such, this method was taken as the starting point for developing a method for separating and detecting ascorbic acid along with the inorganic oxidizers present in these low explosives. Use of a weak anion exchange resin is beneficial because the separation of small and large ions is permitted in a single isocratic run. Given that the $\mathrm{pK}_{\mathrm{a} 1}$ of ascorbic acid is $4.1(110)$, the $\mathrm{pH}$ of the mobile phase had to be sufficiently high to maintain the charge state of the ascorbate ion. At mobile phase $\mathrm{pH}$ greater than 4.2 , baseline separation of the ascorbic acid peak from the 
unretained water peak was not achieved. At $\mathrm{pH}$ values less than 4.2, detection was compromised. An isophthalic acid concentration of $4.5 \mathrm{mM}(34)$ was sufficient for indirect UV detection of the non-absorbing analytes at $280 \mathrm{~nm}$. This system was appropriate for the detection of all target analytes with the exception of perchlorate. This particular ion elutes late in the analysis and can co-elute with carbonate depending on the characteristics and age of the analytical column. Alternative procedures for the detection of perchlorate by ion chromatography include the use of a second ion exchange column (82) or the use of a gradient procedure (11) (14).The separation of a standard anion mixture IC-IPD is illustrated in Figure 4.5. The inorganic anions are each present at 15 ppm, while the organic anion is present at $5 \mathrm{ppm}$.

Figure 4.5. Separation of a standard anion mixture by ion chromatography. Conditions: Waters IC-Pak HR anion exchange column, isocratic elution with $4.5 \mathrm{mM}$ isophthalic acid at $\mathrm{pH} 4.2$, flow rate $1 \mathrm{~mL} / \mathrm{min}, 20 \mu \mathrm{L}$ injection, indirect $\mathrm{UV}$ detection at $280 \mathrm{~nm}$. Peak order: (1) water, Rt 0.99 min (2) ascorbate, Rt $2.12 \mathrm{~min}$ (3) chloride, Rt 3.55 min (4) nitrite, Rt 4.85 min (5) chlorate, Rt 6.53 min (6) nitrate, Rt 7.89 min (7) sulfate, Rt 9.10 $\min (8)$ carbonate/perchlorate, Rt $28.95 \mathrm{~min}$. Baseline resolution is achieved for all standards with the exception of chlorate and nitrate.

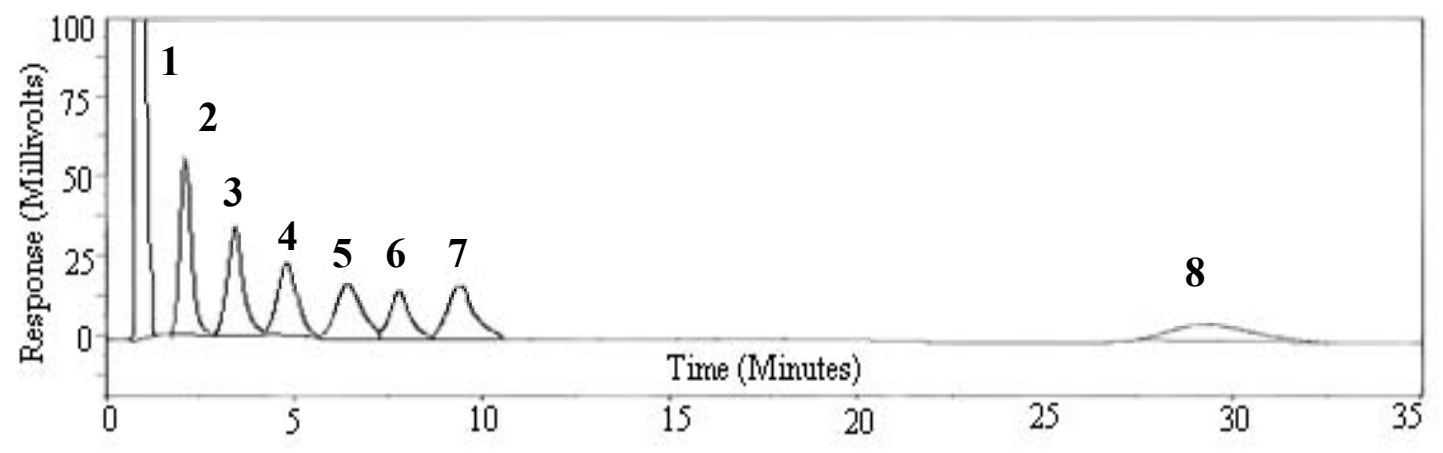




\section{Treatment of ascorbic acid with cysteine}

A solution of ascorbic acid left at room temperature will degrade over a matter of hours to the extent that the original compound can no longer be detected (115). By adding an excess of L-cysteine to the sample, ascorbate can be detected for up to twenty-four hours after entering solution. Figure 4.6 demonstrates the effect of the addition of a complementary amount of cysteine to a sample of ascorbic acid on the ability of the ICIPD system to detect ascorbate.

Figure 4.6. Degradation of ascorbic acid over time with (pink squares) and without (blue diamonds) the addition of an excess of cysteine, as measured by IC-IPD.

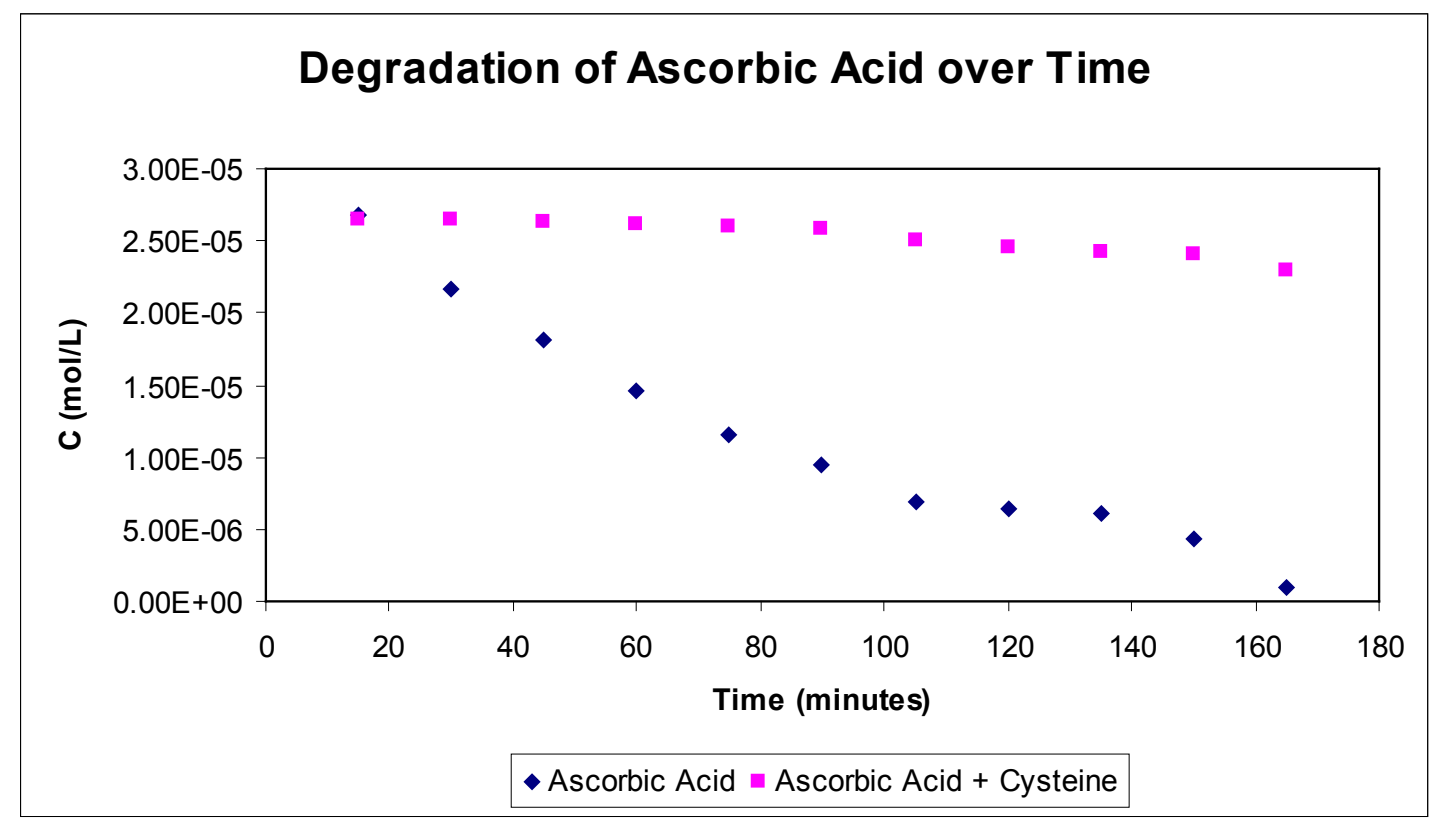

During the course of method development, an excess of L-cysteine was added to all standard solutions of ascorbic acid to prevent its degradation. It was theorized that Lcysteine could be added to water extracts of post-blast propellant samples to preserve 
ascorbate residues for analysis. However, this theory was not tested when it became apparent that storage of the post-blast debris at $\mathrm{T}=-15^{\circ} \mathrm{C}$ from the time of collection to the time of analysis was sufficient to preserve the ascorbic acid residues for separation and detection by IC-IPD.

\section{Validation of IC-IPD Method}

The precision of the retention time of each target anion was determined by performing replicate injections of a standard mixture of $\mathrm{NO}_{2}^{-}, \mathrm{NO}_{3}^{-}, \mathrm{Cl}^{-}, \mathrm{ClO}_{3}^{-}, \mathrm{ClO}_{4}^{-}$, $\mathrm{SO}_{4}{ }^{2-}, \mathrm{HS}^{-}$and ascorbate over a period of three days. Data regarding the average retention time and the relative standard deviation of the retention time for each anion can be viewed in Table 4.2. All of the target anions eluted from the anion exchange column within thirty minutes at a mobile phase flow rate of $1 \mathrm{~mL} / \mathrm{min}$, although perchlorate coeluted with carbonate. The relative standard deviation of the retention times for these analytes ranged from $0.42 \%$ for nitrite to $2.5 \%$ for sulfate.

A concentration curve for each anion was generated on the IC system, using concentrations across the range from $2.5-25 \mu \mathrm{g} / \mathrm{mL}$ for nitrite, nitrate, chloride, and sulfate, from $1.5-25 \mu \mathrm{g} / \mathrm{mL}$ for chlorate, and from 1-5 $\mu \mathrm{g} / \mathrm{mL}$ for ascorbate. Linear regression data were collected from the peak areas of each anion over this limited concentration range. For example, the linear regression equation for nitrite was $\mathrm{y}=26,612 \mathrm{x}+6766$ with an $r^{2}$ value of 0.9995 . The $r^{2}$ values for the other anions ranged from 0.9959 to 0.9995 . Concentrations of ascorbate greater than $5 \mu \mathrm{g} / \mathrm{mL}$ resulted in detector saturation. Inorganic anion concentrations greater than $25 \mu \mathrm{g} / \mathrm{mL}$ resulted in non-uniform peak shapes and ultimately, detector saturation. 
Table 4.2. Figures of merit for IC-IPD. The RSD values for the retention time of the target anions by ion chromatography are low, indicating that the results are reproducible.

\begin{tabular}{|c|c|c|c|}
\hline Ions & Rt (min) & Stnd Dev Rt & $\%$ RSD Rt \\
\hline Ascorbate & 2.16 & 0.03 & 1.2 \\
\hline Chloride & 3.58 & 0.04 & 1.1 \\
\hline Nitrite & 4.81 & 0.02 & 0.41 \\
\hline Chlorate & 6.55 & 0.04 & 0.61 \\
\hline Nitrate & 7.86 & 0.06 & 0.76 \\
\hline Sulfate & 9.07 & 0.23 & 0.29 \\
\hline Hydrogen sulfide & 13.87 & 0.04 & NA \\
\hline Perchlorate & 28.9 & NA & \\
\hline
\end{tabular}

Figure 4.7. Calibration curve data for ascorbate by IC-IPD. The curve is linear over the range $1-5 \mu \mathrm{g} / \mathrm{mL}$.

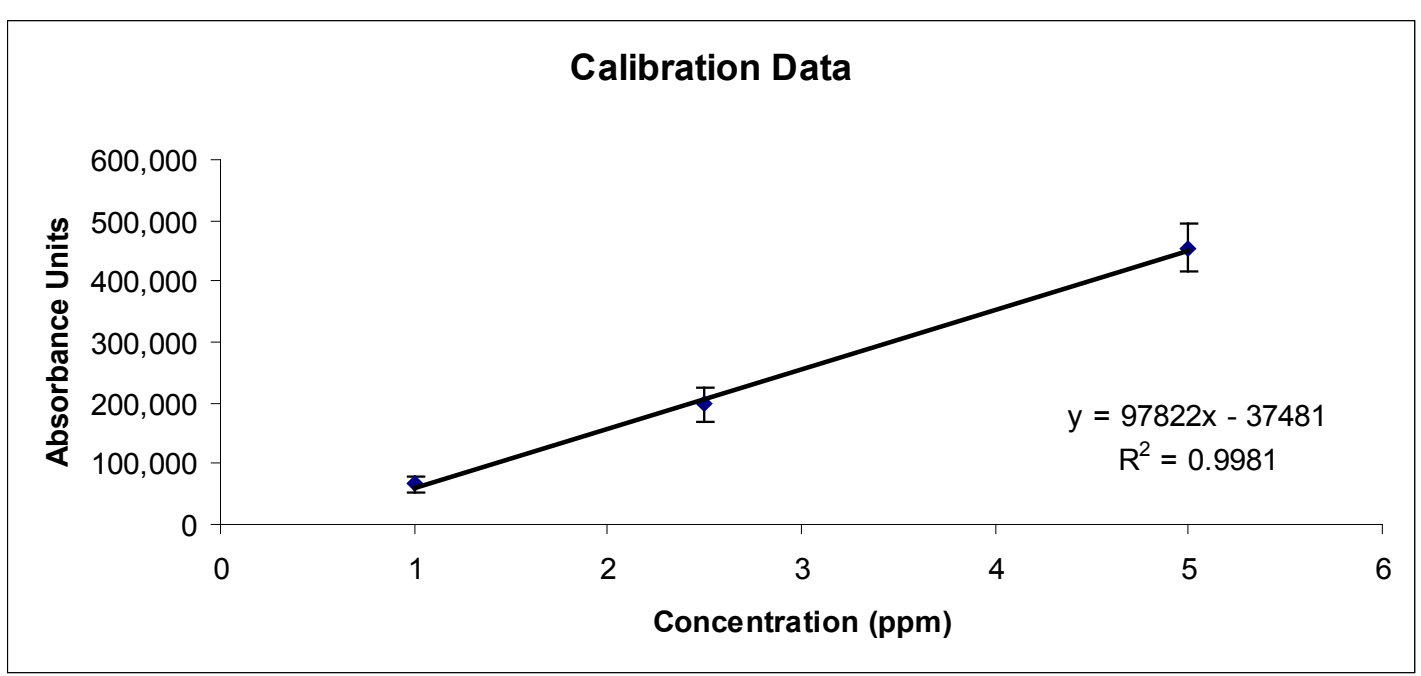


Figure 4.8. Calibration curve data for chloride, nitrite, nitrate, chlorate, and sulfate by IC-IPD. The curves for chloride, nitrite, nitrate, and sulfate are linear over the range of $2.5-25 \mu \mathrm{g} / \mathrm{mL}$, while the curve for chlorate is linear over the range of $1-25 \mu \mathrm{g} / \mathrm{mL}$.

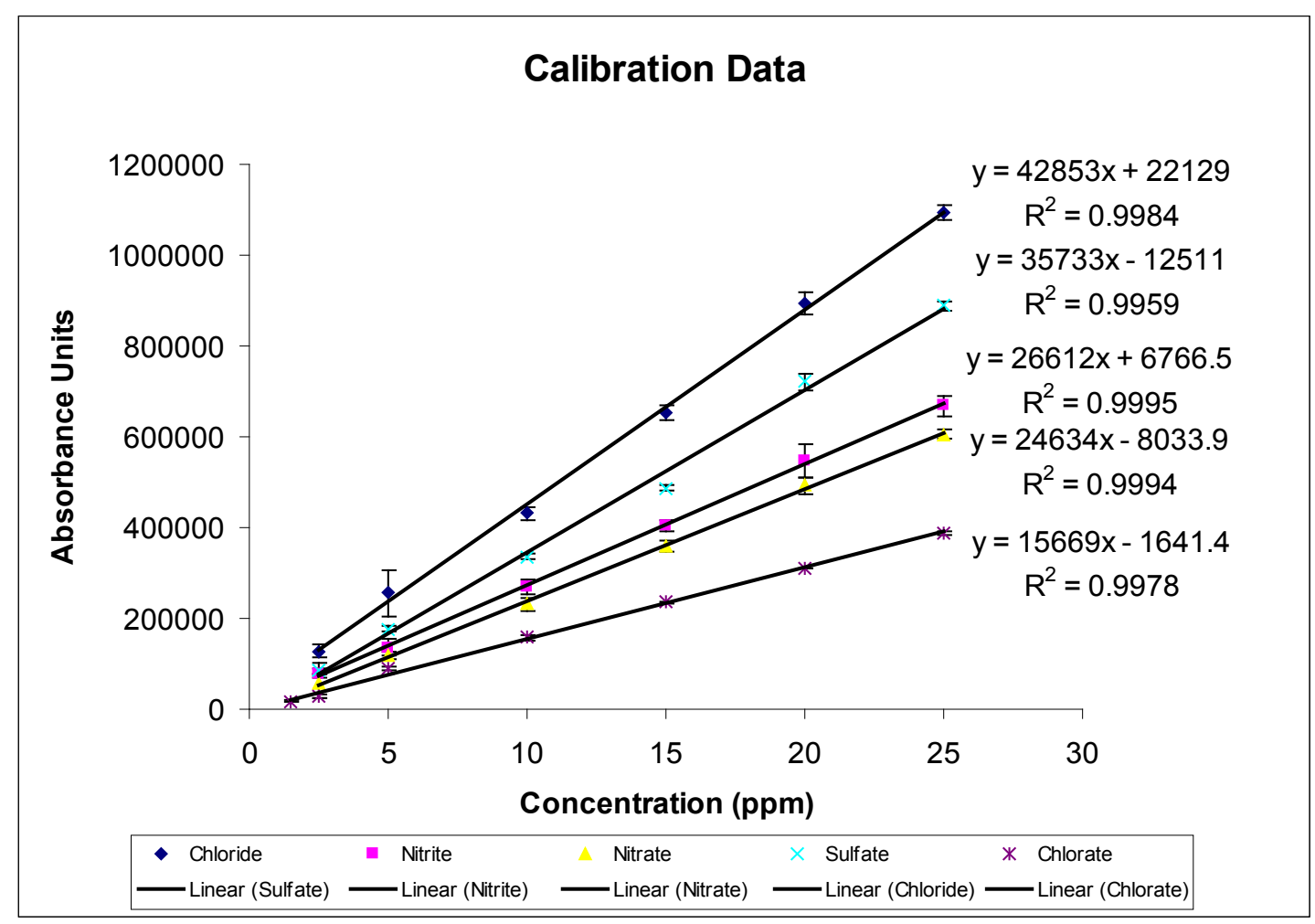

The limit of detection (LOD) is defined as the concentration which gives an instrument signal which is significantly different from the blank or background signal. In practical terms, this can be defined as the analyte concentration giving a signal equal to the blank signal $\left(\mathrm{y}_{\mathrm{B}}\right)$ plus three standard deviations of the blank, $\mathrm{s}_{\mathrm{B}}$ (see below) (116).

$$
\mathrm{y}-\mathrm{y}_{\mathrm{B}}=3 \mathrm{~s}_{\mathrm{B}}
$$

To calculate the limit of detection using this format, ten replicate injections were performed for each anion standard at a concentration which was very near to the theorized instrument detection limit. The average and standard deviation of these 
measurements were determined, and this information was substituted into the above equation. The resulting y-value was then converted to a concentration value; this value corresponded to the limit of detection in terms of sample concentration. The LOD ranged from $0.13 \mathrm{ppm}$ for ascorbate, to $0.81 \mathrm{ppm}$ for chloride (Table 4.3). These data were not available for perchlorate or hydrogen sulfide due to problems with decomposition (hydrogen sulfide) and co-elution (perchlorate).

Table 4.3. Detection limit and linear dynamic range for organic and inorganic anions by IC-IPD. The LODs ranged from $0.13 \mathrm{ppm}$ for ascorbate to $0.81 \mathrm{ppm}$ for chloride. LOD values could not be determined for hydrogen sulfide or perchlorate.

\begin{tabular}{|c|c|c|c|}
\hline \multirow{2}{*}{ Ions } & $\begin{array}{c}\text { Detection Limit } \\
(\mu \mathrm{g} / \mathrm{mL})\end{array}$ & $\begin{array}{c}\text { Linear Dynamic } \\
\text { Range }(\mu \mathrm{g} / \mathrm{mL})\end{array}$ & $\begin{array}{c}\text { Correlation } \\
\text { Coefficient }(R)\end{array}$ \\
\hline Ascorbate & 0.13 & $1.0-5.0$ & 0.9971 \\
\hline Chloride & 0.16 & $2.5-25$ & 0.9984 \\
\hline Nitrite & 0.32 & $2.5-25$ & 0.9995 \\
\hline Chlorate & 0.81 & $1.5-25$ & 0.9978 \\
\hline Nitrate & 0.29 & $2.5-25$ & 0.9994 \\
\hline Sulfate & 0.24 & $2.5-25$ & 0.9959 \\
\hline
\end{tabular}

The effect of interferences upon the analysis and detection of the target analytes was also examined. A group of potential interferences, including iodide, acetate, citrate, formate, cyanide, phosphate, tartrate, oxalate, and bromide were analyzed to determine the degree to which their presence might hinder detection of the organic and inorganic 
ions of interest. The results of this study indicated that several of these compounds interfered with the detection of the anions of interest on the ion chromatography system. Phosphate and acetate co-eluted with ascorbate; tartrate and bromide co-eluted with chlorate; and cyanide co-eluted with carbonate and perchlorate. In the event that such ions are present in the matrix of a post-blast sample, a second technique would be required to resolve the co-eluting materials.

\section{Results of Sample Analysis}

The propellants which were analyzed during this study included Goex Black Powder (grades FF and FFF), Pyrodex (grades RS and P), American Pioneer (grades FF and FFF), Jim Shockey's Gold (grades FF and FFF), Triple 7 (grade FFF), Black Mag 3, and Clean Shot. Extracts of intact explosives were prepared by dissolving twenty milligrams of each propellant in $1.0 \mathrm{~mL}$ of $18.3 \mathrm{M} \Omega$ DI water, and sonicating for twenty minutes. Prior to analysis, the extracts were filtered and diluted by bringing $1.0-5.0 \mu \mathrm{L}$ of the original extract to a volume of $1.00 \mathrm{~mL}$ in $18.3 \mathrm{M} \Omega$ DI water. Each sample was analyzed on a Waters IC-Pak HR anion exchange column with isocratic elution at $4.5 \mathrm{mM}$ isophthalic acid at $\mathrm{pH}$ 4.2. The flow rate was $1 \mathrm{~mL} / \mathrm{min}$ with a $20 \mu \mathrm{L}$ injection volume. Indirect UV detection at $280 \mathrm{~nm}$ was utilized. Results from the analysis of a sample of intact American Pioneer powder are presented in Figure 4.9. Results from the analysis of a sample of intact Jim Shockey's Gold are presented in Figure 4.10. A summary of the results of the analyses of the intact propellants can be found in Table 4.4. 
Figure 4.9. Analysis of intact American Pioneer powder. Conditions: Waters IC-Pak HR anion exchange column, isocratic elution with $4.5 \mathrm{mM}$ isophthalic acid at $\mathrm{pH} 4.2$, flow rate $1 \mathrm{~mL} / \mathrm{min}, 20 \mu \mathrm{L}$ injection, indirect UV detection at $280 \mathrm{~nm}$. Peak order: (1) water, Rt $0.97 \mathrm{~min}(2)$ ascorbate, Rt $2.19 \mathrm{~min}$ (3) nitrate, Rt $7.81 \mathrm{~min}$ (4) perchlorate/carbonate $29.0 \mathrm{~min}$.

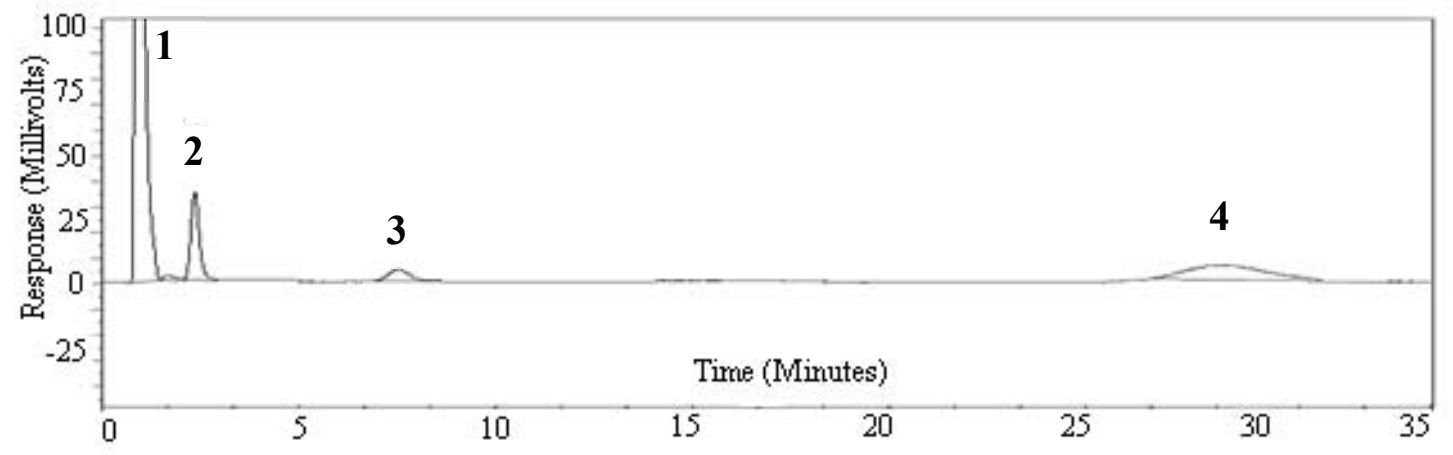

Figure 4.10. Analysis of intact Jim Shockey's Gold powder. Conditions: Waters IC-Pak HR anion exchange column, isocratic elution with $4.5 \mathrm{mM}$ isophthalic acid at $\mathrm{pH} 4.2$, flow rate $1 \mathrm{~mL} / \mathrm{min}, 20 \mu \mathrm{L}$ injection, indirect $\mathrm{UV}$ detection at $280 \mathrm{~nm}$. Peak order: (1) water, Rt $1.00 \mathrm{~min},(2)$ ascorbate, Rt $2.19 \min (3)$ nitrate, Rt $7.82 \min (4)$ perchlorate/carbonate $28.95 \mathrm{~min}$.

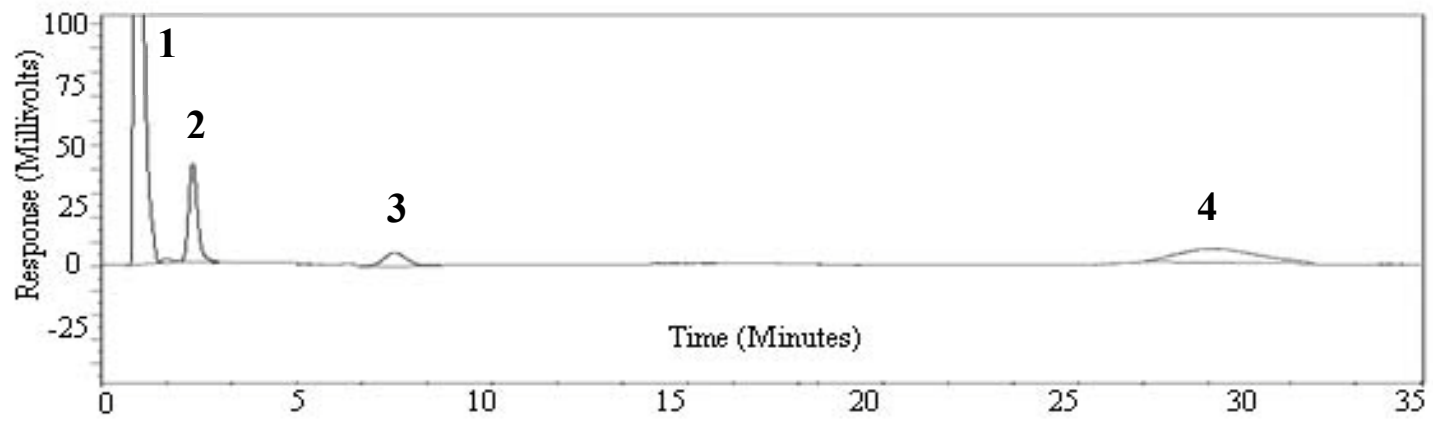


Table 4.4. Results of analysis of intact propellant samples. Ascorbate was detected in all four of the ascorbic acid-based propellants.

\begin{tabular}{|c|c|c|c|c|c|c|c|c|}
\hline Propellant & $\mathrm{AA}$ & $\mathrm{Cl}$ & $\mathrm{NO}_{2}^{-}$ & $\mathrm{ClO}_{3}^{-}$ & $\mathrm{NO}_{3}^{-}$ & $\mathrm{HSO}_{4}^{-}$ & $\mathrm{HS}$ & $\mathrm{ClO}_{4}^{-} / \mathrm{CO}_{3}{ }^{2-}$ \\
\hline AMP 2F & Yes & No & No & No & Yes & No & No & Co-elution \\
\hline AMP 3F & Yes & No & No & No & Yes & No & No & Co-elution \\
\hline BM3 & Yes & No & No & No & Yes & No & No & Co-elution \\
\hline CS & Yes & No & No & No & Yes & No & No & Co-elution \\
\hline GBP 3F & No & No & No & No & Yes & No & No & Co-elution \\
\hline JSG 2F & Yes & No & No & No & Yes & No & No & Co-elution \\
\hline JSG 3F & Yes & No & No & No & Yes & No & No & Co-elution \\
\hline PYR P & No & No & No & No & Yes & No & No & Co-elution \\
\hline PYR RS & No & No & No & No & Yes & No & No & Co-elution \\
\hline 777 & No & No & No & No & Yes & No & No & Co-elution \\
\hline
\end{tabular}

In viewing the results displayed in Table 4.4, it is clear that the ascorbic acid fuel was detected as the ascorbate anion in intact samples of American Pioneer, Clean Shot, Black Mag3, and Jim Shockey's Gold. The nitrate oxidizer was also detected in these propellants. The presence of perchlorate could not be confirmed because of co-elution issues with carbonate. Chlorate, nitrite, sulfate, and hydrogen sulfide were absent from these samples. This was to be expected, as chlorate and nitrite are decomposition products of perchlorate and nitrate, which typically only appear when the propellant has been burned or otherwise degraded. Sulfate and hydrogen sulfide were also not expected 
to be present, as these brands of propellant do not contain sulfur. The only ion detected in extracts from the Goex Black Powder, Pyrodex, and Triple7 propellant samples was nitrate. These results are expected, as these propellants include a charcoal-based fuel source rather than ascorbic acid. Although sulfur is an ingredient in both Goex Black Powder and Pyrodex, neither sulfate nor hydrogen sulfide ions were noted in the extracts of the intact propellants. This is also to be expected, as sulfate and sulfide ions only appear upon burning of the propellants. The presence of perchlorate in Pyrodex and Triple7 could not be confirmed because of the problem of co-elution with carbonate.

Burned samples were prepared by placing 100 milligrams of each propellant on a clean, dry watch glass and deflagrating them with a propane torch. The burned residues were dissolved in $1.00 \mathrm{~mL}$ of $18.3 \mathrm{M} \Omega \mathrm{DI}$ water and allowed to sit on the watch glass for five minutes. The extracts were filtered prior to analysis. Each sample was analyzed on a Waters IC-Pak HR anion exchange column with isocratic elution at $4.5 \mathrm{mM}$ isophthalic acid at $\mathrm{pH}$ 4.2. The flow rate was $1 \mathrm{~mL} / \mathrm{min}$ with a $20 \mu \mathrm{L}$ injection volume. Indirect $\mathrm{UV}$ detection at $280 \mathrm{~nm}$ was utilized. Results from the analysis of a sample of burned Jim Shockey's Gold powder are presented in Figure 4.11. Results from the analysis of a sample of burned American Pioneer powder are presented in Figure 4.12. Results from the analysis of a sample of burned Triple7 powder are presented in Figure 4.13. A summary of the results of the analyses of the intact propellants can be found in Table 4.5. 
Figure 4.11. Analysis of burned Jim Shockey's Gold powder. For conditions, see text. Peak Order: (1) water, Rt $1.05 \mathrm{~min}$ (2) ascorbate, Rt $2.14 \mathrm{~min}$ (3) chloride, Rt $3.63 \mathrm{~min}$ (4) nitrite, Rt $4.81 \mathrm{~min}$ (5) unknown, Rt $5.21 \mathrm{~min}$ (6) chlorate, Rt $6.55 \mathrm{~min}$ (7) nitrate, Rt $7.89 \mathrm{~min}(8)$ perchlorate/carbonate $29.22 \mathrm{~min}$.

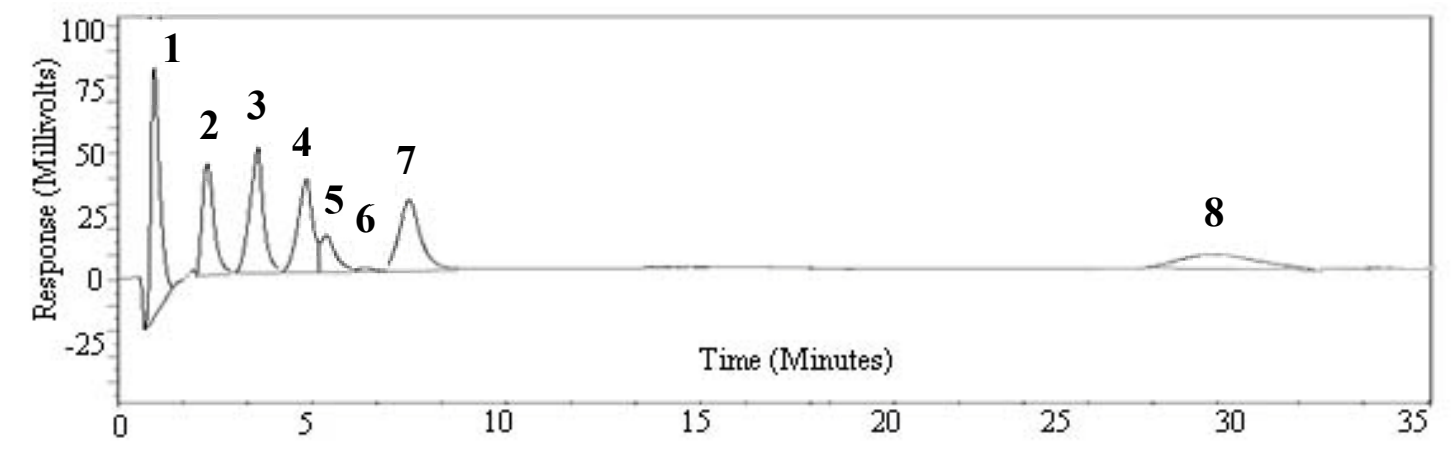

Figure 4.12. Analysis of burned American Pioneer powder. For conditions, see text. Peak order: (1) water, Rt $1.00 \mathrm{~min}$ (2) ascorbate, Rt $2.16 \mathrm{~min}$ (3) chloride, Rt $3.59 \mathrm{~min}$ (4) nitrite, Rt 4.85 min (5) unknown, Rt 5.22 min (6) chlorate, Rt 6.62 min (7) nitrate, Rt $7.86 \mathrm{~min}(8)$ perchlorate/carbonate $29.17 \mathrm{~min}$.

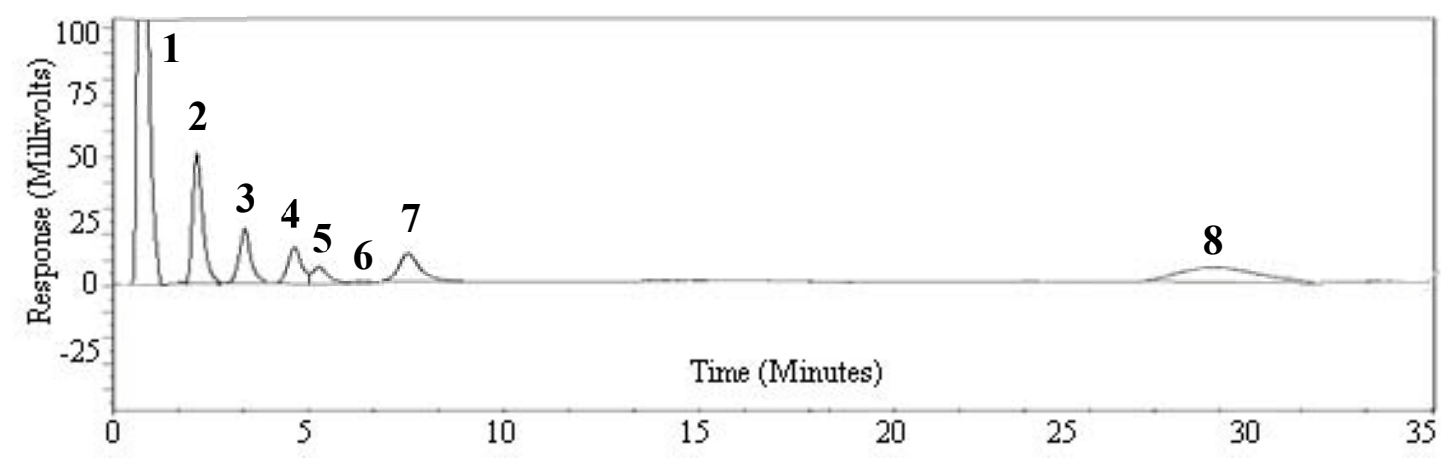


Figure 4.13. Analysis of burned Triple7 powder. For conditions, see text. Peak order: (1) water, Rt $0.99 \min (2)$ chloride, Rt 3.56 min (3) nitrite, Rt 4.77 min (4) unknown, Rt 5.18 $\min (5)$ nitrate, Rt 7.83 min (6) perchlorate/carbonate 28.95.

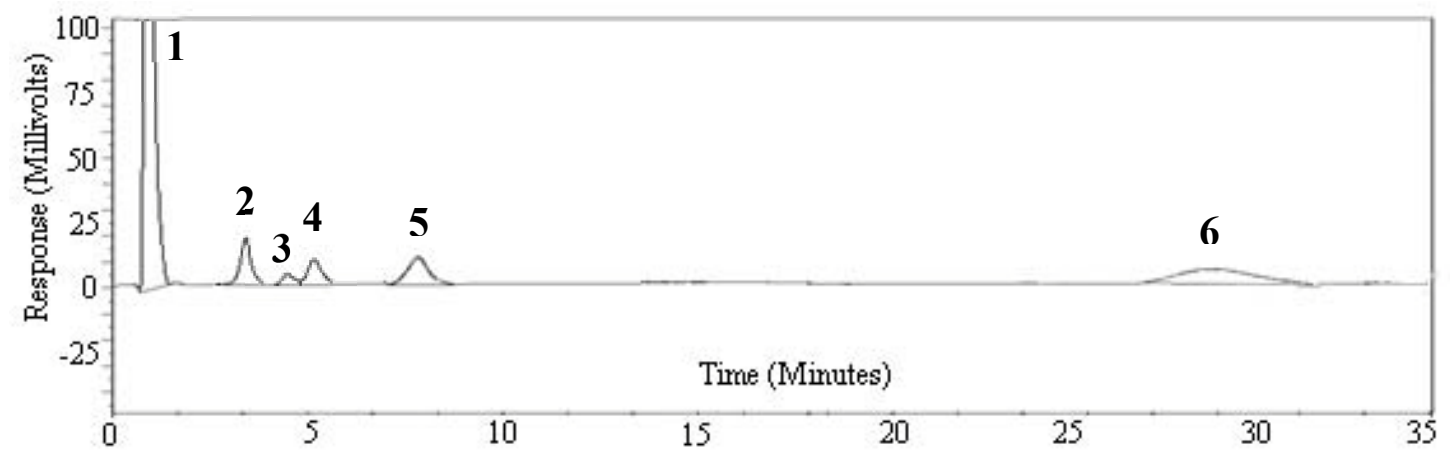

In viewing Table 4.5, it is apparent that ascorbate was detected in the extracts from the post-burn residue of three of the four brands of ascorbic acid-based propellants: American Pioneer, Black Mag3, and Jim Shockey’s Gold. Ascorbate was not detected in the extract from the post-burn residue of Clean Shot. It should be noted that Clean Shot is an older ascorbic acid-based propellant which has not been in production for at least a decade. The effects of time and humidity were clearly evident in the slower burn rate of the powder, and general state of clumping of the propellant in the original container from the manufacturer. Exposure to humidity may have accelerated the rate at which the ascorbic acid degraded in the bottle. The failure to detect ascorbate in the post-burn Clean Shot samples could be related to this issue. Also present in the post-burn reside of the ascorbic acid-containing propellants were chloride, chlorate, nitrate, and nitrite. This was to be expected as chloride and chlorate are decomposition products of the perchlorate oxidizer, and nitrite is a decomposition product of the nitrate oxidizer. The presence of 
perchlorate in the post-burn residue could not be confirmed due to the problem of coelution with carbonate.

Table 4.5. Results of analysis of burned propellant samples. Ascorbate was detected in the post-burn residues of three out of four brands of ascorbic acid-based propellants.

\begin{tabular}{|c|c|c|c|c|c|c|c|c|}
\hline Propellant & $\mathrm{AA}$ & $\mathrm{Cl}^{-}$ & $\mathrm{NO}_{2}^{-}$ & $\mathrm{ClO}_{3}^{-}$ & $\mathrm{NO}_{3}^{-}$ & $\mathrm{HSO}_{4}^{-}$ & $\mathrm{HS}^{-}$ & $\mathrm{ClO}_{4}^{-} / \mathrm{CO}_{3}^{2-}$ \\
\hline AMP 2F & Yes & Yes & Yes & Yes & Yes & No & No & Co-elution \\
\hline AMP 3F & Yes & Yes & Yes & Yes & Yes & No & No & Co-elution \\
\hline BM3 & Yes & Yes & Yes & Yes & Yes & No & No & Co-elution \\
\hline GBP 2F & No & Yes & Yes & Yes & Yes & No & No & Co-elution \\
\hline GBP 3F & No & No & Yes & No & Yes & Yes & Yes & Co-elution \\
\hline JSG 2F & Yes & Yes & Yes & Yes & Yes & No & No & Co-elution \\
\hline JSG 3F & Yes & Yes & Yes & Yes & Yes & No & No & Co-elution \\
\hline PYR P & No & Yes & Yes & Yes & Yes & Yes & Yes & Co-elution \\
\hline PYR RS & No & Yes & Yes & Yes & Yes & Yes & Yes & Co-elution \\
\hline 777 & No & Yes & Yes & Yes & Yes & No & No & Co-elution \\
\hline
\end{tabular}

As expected, ascorbate was not detected in the extracts from the post-burn residue of the charcoal based powders. Chlorate, chloride, nitrate, and nitrite were present in the extract from the post-burn residue of Triple7. The extract from the post-burn residue of Pyrodex included chlorate, chloride, nitrate, nitrite, sulfate, and sulfide. The presence of perchlorate in the post-burn residue of these two propellants could not be confirmed due 
to the problem of co-elution with carbonate. The extract from the post-burn residue of Goex Black Powder included nitrate, nitrite, sulfate, and sulfide. These results are in keeping with information regarding the composition of Pyrodex, Triple7, and Goex Black Powder.

In viewing Figures $4.11,4.12$, and 4.13 , there is a peak of unknown composition which appears at approximately 5.15 minutes. This peak was not present in the extracts collected from the intact propellants samples. It appears that the compound which gives rise to this peak exists as a result of the burning process. It is unlikely that this peak corresponds to degradation products of ascorbic acid because this peak was present in the post-burn samples of the charcoal-based propellants as well as the ascorbic acid-based propellants. This peak was not present in the material blanks, so it is unlikely that it is a contaminant. The identity of this peak remains unknown at this time.

Figure 4.14 presents the relative abundance of select anionic residues present in the post-burn and post-blast black powder substitute samples. Only those anions with concentration curves which were linear over the range of 2.5-25 ppm (inorganic) or 1-5 ppm (organic) were included in the relative abundance graphs; namely chloride, nitrite, chlorate, nitrate, sulfate, and ascorbate. Although hydrogen sulfide was detected in the Pyrodex and Goex Black Powder samples, it could not be reliably quantified for inclusion in the data set. The relative abundance of each anion was calculated by dividing the amount of the anion present in the sample by the total amount of anionic material detected.

From viewing Figure 4.14, it is apparent that sulfate had the highest relative abundance for the non-ascorbic acid-based propellants, followed in most cases by nitrate. 
This creates a pattern which is indicative of charcoal/sulfur based propellants. For the ascorbic acid-based propellants, nitrate had the highest relative abundance, followed by nitrite and ascorbate. The lack of sulfate indicates that these propellants are not charcoal/sulfur based. The presence of ascorbate clearly identifies the ascorbic acid-base propellants as containing that particular fuel. However, in the case of Clean Shot, the lack of ascorbate and presence of abundant levels of nitrate, nitrite, and chlorate make it difficult to distinguish between it and Triple7.

Figure 4.14. Relative abundance of residues present in burned propellant samples. The relative abundance is the percentage of each anion in the sample. Nitrate is the most abundant ion present in the post-burn residues of the ascorbic acid-based propellants, while sulfate is the most abundant ion present in the post-burn residues of Pyrodex and Goex Black Powder.

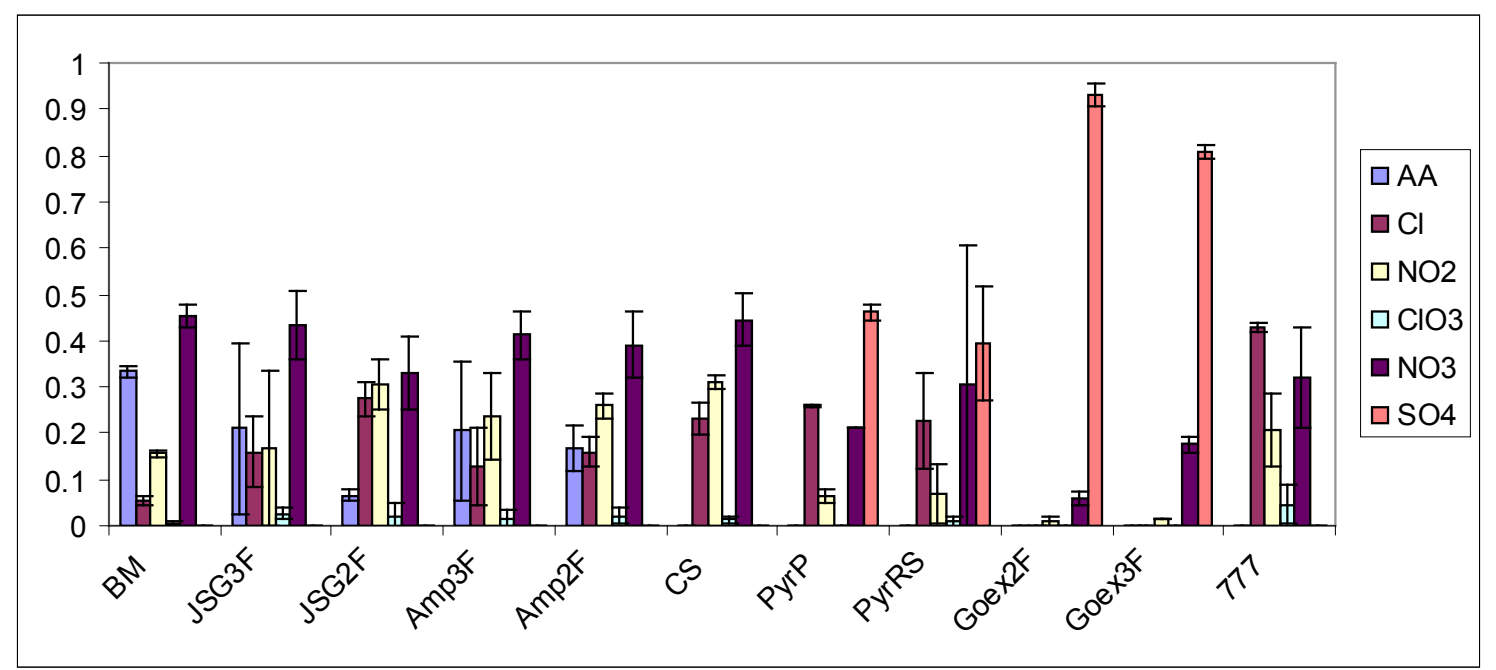


A pipe bomb study was conducted to examine differences in the quantity and type of anionic residues produced by various black powder substitutes. The IED study utilized thirty-three polyvinyl chloride (PVC) pipes filled with explosive material that was deflagrated by the Miami Dade Police Department Bomb Squad. Seven different brands of black powder and black powder substitutes were utilized in the study. In addition, four of the propellants were obtained in two different grain sizes, resulting in a total of eleven different types of powder. Each powder was analyzed in triplicate for a total of thirtythree shots. Initiation of each explosive charge was performed via an electric match. Each individual pipe was suspended within its own plastic bucket to minimize contamination and maximize pipe fragment recovery. Cotton pads which had been previously cleaned with replicate washes of isopropanol and water were employed for residue collection. The interior of each bucket was dry swabbed as a control prior to deflagration. Post-blast residues were collected from inside the bucket with two additional dry swabs; pipe fragments which remained within the bucket were also collected. Each item was packaged separately to prevent cross-contamination. Each sample was analyzed on a Waters IC-Pak HR anion exchange column with isocratic elution at $4.5 \mathrm{mM}$ isophthalic acid at $\mathrm{pH}$ 4.2. The flow rate was $1 \mathrm{~mL} / \mathrm{min}$ with a $20 \mu \mathrm{L}$ injection volume. Indirect $\mathrm{UV}$ detection at $280 \mathrm{~nm}$ was utilized. Results of the analysis of swabs from one of the American Pioneer pipe shots are presented in Figure 4.15. Results from the analysis of swabs from one of Clean Shot pipe shots are presented in Figure 4.16. Results from the analysis of swabs from one of the Black Mag pipe shots are presented in Figure 4.17. The results of this experiment are summarized in Table 4.6. 
Figure 4.15. Analysis of post-blast American Pioneer pipe shot. Conditions: Waters ICPak HR anion exchange column, isocratic elution with $4.5 \mathrm{mM}$ isophthalic acid at $\mathrm{pH} 4.2$, flow rate $1 \mathrm{~mL} / \mathrm{min}, 20 \mu \mathrm{L}$ injection, indirect UV detection at $280 \mathrm{~nm}$. Peak order:

(1) water, Rt $1.00 \mathrm{~min}$ (2) ascorbate, Rt $2.12 \mathrm{~min}$ (3) chloride, Rt $3.54 \mathrm{~min}$ (4) nitrite, Rt $4.79 \mathrm{~min}(5)$ nitrate, Rt $7.88 \mathrm{~min}$ (6) perchlorate/carbonate $29.15 \mathrm{~min}$.

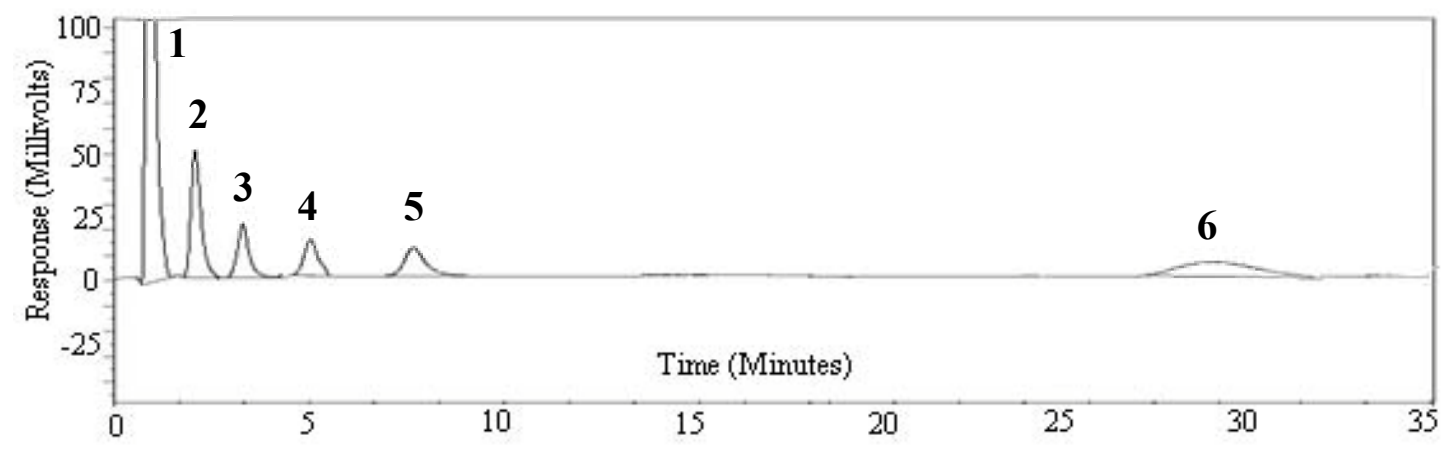

Figure 4.16. Analysis of post-blast Clean Shot pipe shot. Conditions: Waters IC-Pak HR anion exchange column, isocratic elution with $4.5 \mathrm{mM}$ isophthalic acid at $\mathrm{pH} 4.2$, flow rate $1 \mathrm{~mL} / \mathrm{min}, 20 \mu \mathrm{L}$ injection, indirect $\mathrm{UV}$ detection at $280 \mathrm{~nm}$. Peak order (1) water, Rt $0.99 \mathrm{~min}$ (2) ascorbate, Rt $2.14 \mathrm{~min}$ (3) chloride, Rt $3.62 \mathrm{~min}$ (4) nitrite, Rt $4.81 \mathrm{~min}$ (5) nitrate, Rt 7.90 min (6) perchlorate/carbonate $29.24 \mathrm{~min}$

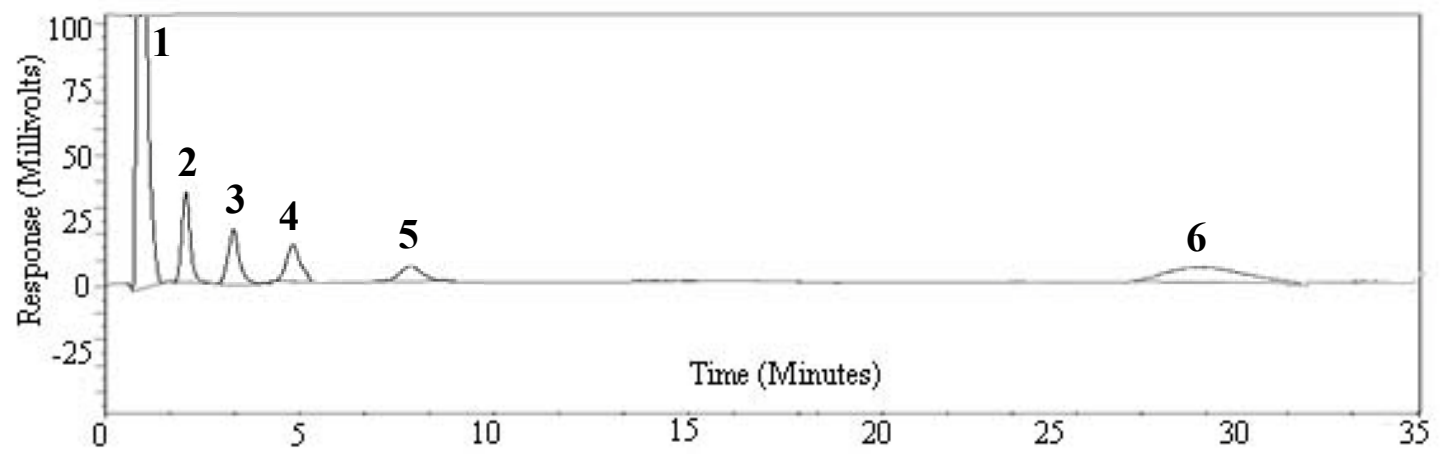


Figure 4.17. Analysis of post-blast Black Mag3 pipe shot. Conditions: Waters IC-Pak $\mathrm{HR}$ anion exchange column, isocratic elution with $4.5 \mathrm{mM}$ isophthalic acid at $\mathrm{pH} 4.2$, flow rate $1 \mathrm{~mL} / \mathrm{min}, 20 \mu \mathrm{L}$ injection, indirect UV detection at $280 \mathrm{~nm}$. Peak order: (1) water, Rt $0.99 \mathrm{~min}$ (2) ascorbate, Rt $2.16 \mathrm{~min}$ (3) chloride, Rt $3.55 \mathrm{~min}$ (4) nitrite, Rt $4.75 \mathrm{~min}(5)$ nitrate, Rt $7.90 \mathrm{~min}$ (6) perchlorate/carbonate $28.99 \mathrm{~min}$.

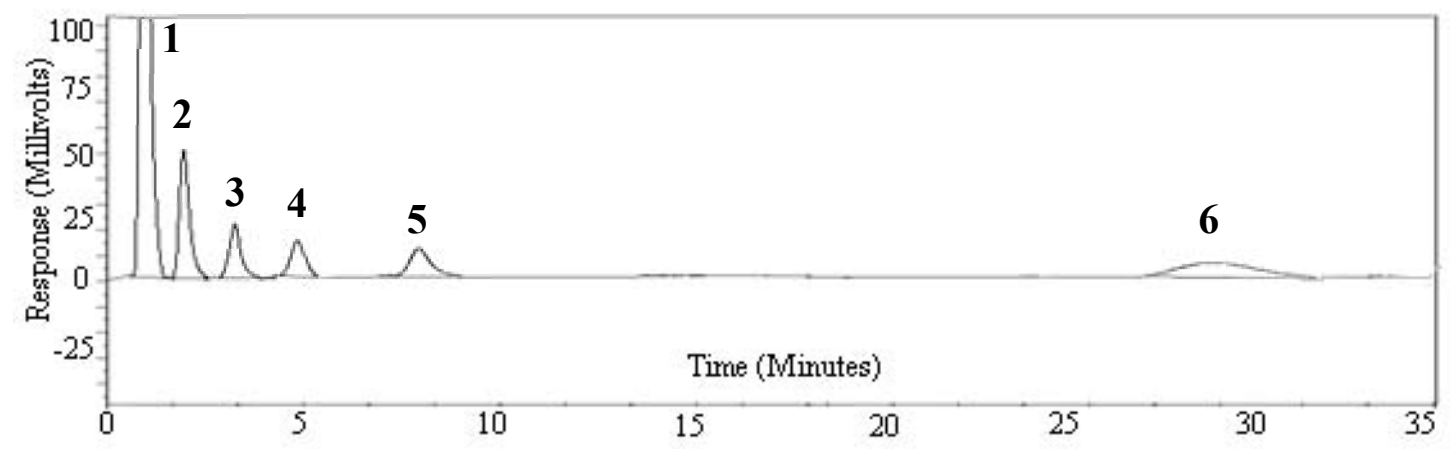

In viewing Table 4.6, it is apparent that ascorbate was detected in the extracts of the post-blast residue from all four brands of ascorbic acid-based propellants. Even though ascorbate was not detected in the extract from the post-burn residue of Clean Shot, the fuel compound was detected in the extract from the post-blast sample. At the time of these experiments, it was unknown whether this fuel source could survive the heat and pressure of an explosion and the extraction process. Prompt refrigeration of the collected samples may have helped in preventing the decomposition of ascorbic acid post-blast, thereby increasing the period in which the samples could be analyzed. Certain post-blast ascorbic acid based propellant samples were not analyzed until eight months after the pipe bombs were deflagrated. Even after this lengthy passage of time, these samples still contained detectable levels of ascorbate. 
Table 4.6. Results of analysis of post-blast propellant samples. Ascorbate was detected in the post-blast residue of all four brands of ascorbic-acid based propellants.

\begin{tabular}{|c|c|c|c|c|c|c|c|c|}
\hline Propellant & $\mathrm{AA}$ & $\mathrm{Cl}^{-}$ & $\mathrm{NO}_{2}^{-}$ & $\mathrm{ClO}_{3}^{-}$ & $\mathrm{NO}_{3}^{-}$ & $\mathrm{HSO}_{4}^{-}$ & $\mathrm{HS}$ & $\mathrm{ClO}_{4}^{-} / \mathrm{CO}_{3}{ }^{-2}$ \\
\hline AMP 2F & Yes & Yes & Yes & No & Yes & No & No & Co-elution \\
\hline AMP 3F & Yes & Yes & Yes & Yes & Yes & No & No & Co-elution \\
\hline CS & Yes & Yes & Yes & Yes & Yes & No & No & Co-elution \\
\hline GBP 2F & No & Yes & Yes & No & Yes & Yes & Yes & Co-elution \\
\hline GBP 3F & No & Yes & Yes & No & Yes & Yes & Yes & Co-elution \\
\hline JSG 2F & Yes & Yes & Yes & No & Yes & No & No & Co-elution \\
\hline JSG 3F & Yes & Yes & Yes & No & Yes & No & No & Co-elution \\
\hline PYR P & No & Yes & Yes & No & Yes & Yes & Yes & Co-elution \\
\hline PYR RS & No & Yes & Yes & No & Yes & Yes & Yes & Co-elution \\
\hline 777 & No & Yes & Yes & Yes & Yes & No & No & Co-elution \\
\hline
\end{tabular}

Also present in the post-blast residue of the ascorbic acid-based propellants were chloride, nitrate, and nitrite. This was to be expected as nitrite is a decomposition product of the nitrate oxidizer, while chloride is decomposition product of both the perchlorate oxidizer and the polyvinyl chloride pipe from which the devices were constructed. In fact, chloride was detected in the extracts from the post-blast residue of all of the propellants, even those which did not contain a perchlorate oxidizer. It is interesting to note that chlorate was only detected in the three of the nine propellants which contained a perchlorate oxidizer: Black Mag3, American Pioneer FFF, and Triple7. It is possible that 
during deflagration, the majority of the perchlorate was rapidly consumed, resulting in the production of minimal amounts of chlorate. Because the PVC pipe was also a source of chloride, no conclusions can be drawn regarding the production of chloride residue from the consumption of perchlorate. The presence of perchlorate in the post-blast residues of these propellants could not be confirmed due to the problem of co-elution with carbonate.

Chloride, nitrate, and nitrite were present in the post-blast residue of the charcoalbased propellants Pyrodex, Triple7, and Goex Black Powder. As expected, ascorbate was not detected in these samples. Abundant levels of sulfate were present in the post-blast samples of the sulfur containing propellants Pyrodex and Goex Black Powder. Also present was hydrogen sulfide, but the levels of this anion could not be reliably quantified for inclusion in the graph. These results are in keeping with the results obtained from analysis of the extracts from the burned propellants.

Figure 4.18 presents the relative abundance of select anionic residues present in the post-burn and post-blast black powder substitute samples. Only those anions with concentration curves which were linear over the range of 2.5-25 ppm (inorganic) or 1-5 ppm (organic) were included in the relative abundance graphs: chloride, nitrite, chlorate, nitrate, sulfate, and ascorbate. The relative abundance of each anion was calculated by dividing the amount of the anion present in the sample by the total amount of anionic material detected.

The most abundant anion present in the post-blast ascorbic acid-based propellants was nitrate, followed by nitrite. While nitrate was also present in the post-burn samples, a comparison of the post-burn and post-blast graphs reveals that this ion is present in higher 
levels in post-blast samples. One possibility for this observation is that deflagration occurred more rapidly in the post-blast samples, leading to the chance that relatively more unburned material may have been left behind. The presence of ascorbate clearly identifies the ascorbic acid-base propellants as containing that particular fuel. From viewing Figure 4.18, it is apparent that sulfate had the highest relative abundance for the non-ascorbic acid-based propellants, followed in all cases by nitrate. This again creates a pattern which is indicative of charcoal/sulfur based propellants.

Figure 4.18. Relative abundance of residues present in post-blast propellant samples. The relative abundance is the percentage of each anion in the sample. Nitrate is the most abundant anion in the post-blast residue of the ascorbic-acid based propellants.

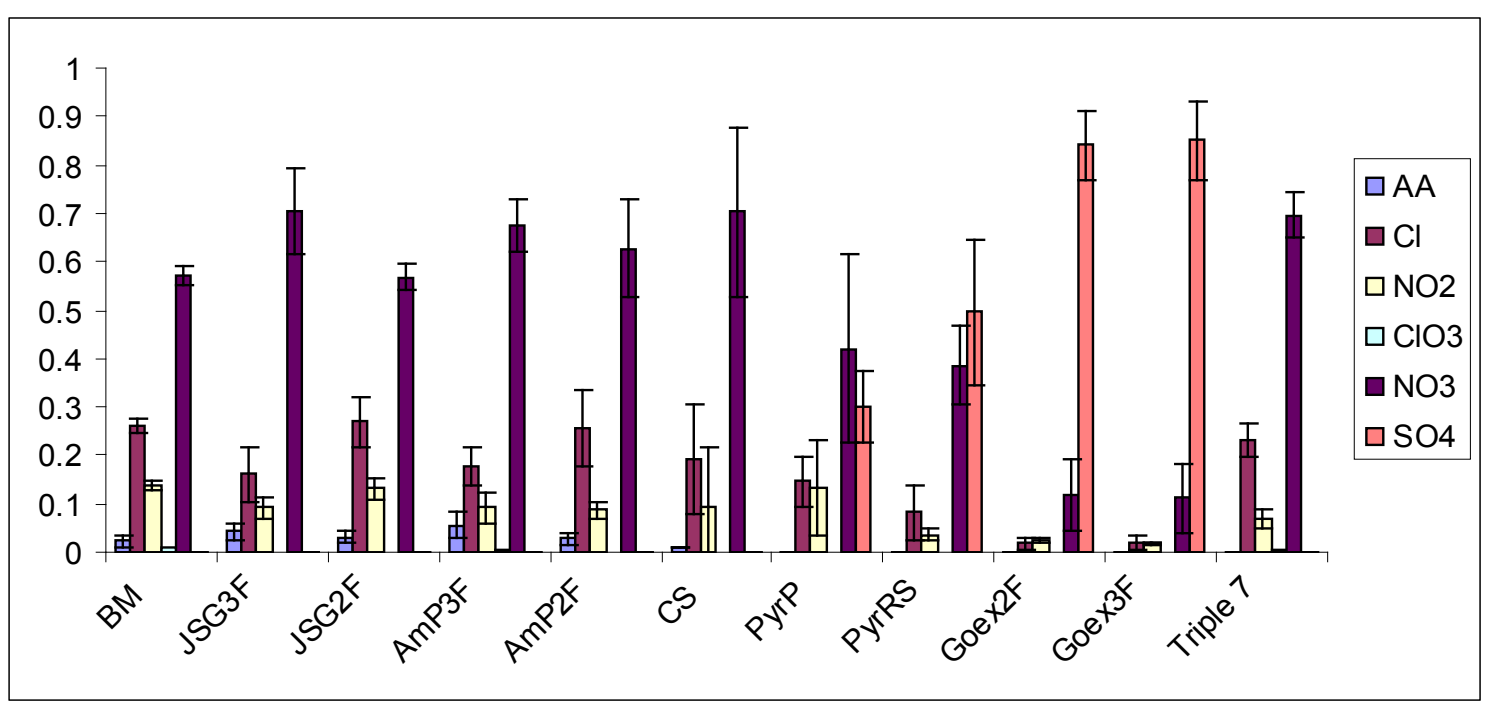

The presence and amount of specific residues in a post-blast sample can vary considerably under a given set of conditions; the variation is even greater when conditions are changed. It is entirely possible that changing select factors of the IED 
experiment such as the size or type of containment vessel, the amount of propellant used, or the type if initiator, could result in the formation of post-blast samples with different relative abundances of key residues. It would be interesting to repeat this study using metal pipes in place of PVC; perhaps the chloride residues could then be included in the data set to help discriminate between propellant samples. It would also permit the comparison of perchlorate, chlorate, and chloride residues. It is possible that these data could be used to determine the relative amount of intact propellant which remained, as a measure of blast efficiency.

\section{E. Conclusions}

The IC-IPD method optimized for the analysis of ascorbic acid provides a rapid and efficient method for determining whether a particular propellant is an ascorbic acid-based low explosive. The ascorbic acid fuel, the nitrate oxidizer, and a variety of degradation products from the nitrate and perchlorate oxidizers can be detected. With this approach, not only can the ascorbic acid based powders be distinguished from charcoal based powders in pristine, unburned samples, but this determination can also be made in postburn and post-blast samples. At this time, different brands of ascorbic acid containing propellants cannot be distinguished from one another using these analytical techniques; nor can one grain size of powder be distinguished from another. However, the method presented in this chapter provides a rapid and efficient method for the analysis of ascorbic acid containing black powder substitutes. 


\section{Chapter V. Analysis of Ascorbic Acid-Based Propellants by HPLC-ESI- QTOFMS}

\section{A. Introduction}

High performance liquid chromatography-electrospray ionization-quadrupoletime-of-flight mass spectrometry (HPLC-ESI-QToFMS) is a powerful tool for the analysis of small ions. The use of a QToF mass spectrometer provides accurate mass measurements of target anions with a precision of $\pm 5 \mathrm{ppm}$ or better, providing important information on potential elemental composition. HPLC-ESI-QToFMS also permits the collection of retention time data and mass spectral fragmentation patterns. The totality of these data can be utilized to provide an unequivocal identification of ions of interest. Additionally, both organic and inorganic species can be analyzed with this approach, making it an excellent method for the analysis of black powder substitutes. This technology can therefore be utilized to determine the class of propellant from both bulk powders and post-blast residues.

Previous studies have demonstrated the feasibility of examining select components of ascorbic acid-based black powder substitutes by gas chromatographymass spectrometry (GC-MS) (89), high performance liquid chromatography-mass spectrometry (HPLC-MS) (80) (90), ion chromatography with indirect photometric detection (IC-IPD) (117), and ion chromatography-mass spectrometry (IC-MS) (82). In the first of these studies, Goodpaster and Keto (89) developed a gas chromatographymass spectrometry (GC-MS) method for the analysis of the organic portion of ascorbic acid based propellants. This approach required derivatization of the fuel compounds with N,O-bis(trimethylsilyl)acetamide. This method successfully detected ascorbic acid and its 
degradation products in the tested propellants, but no information about the inorganic portion of the explosive could be ascertained. Studies by Dreifuss et al. (80) (90) resulted in the development of a reversed phase high performance liquid chromatography-mass spectrometry (HPLC-MS) method for the analysis of organic and selected inorganic components of these propellants. This approach permitted the detection of the fuel compounds ascorbic acid, benzoic acid, and dicyandiamide, and the oxidizer compounds perchlorate and nitrate. This method was applied to the analysis of intact powders only. No post-blast studies of ascorbic acid-based propellants were conducted. In addition, this procedure lacked the ability to detect decomposition products of ascorbic acid.

Bottegal and McCord (117) developed a method for the simultaneous analysis of the anionic form of ascorbic acid (ascorbate) and select inorganic anions by ion chromatography with indirect photometric detection (IC-IPD). Ascorbate was detected by IC-IPD in intact, post-burn, and post-blast propellant samples. However, perchlorate could not be reliably detected by IC-IPD. Another drawback to this method is that the technique was insufficient by itself to determine the identity of the components with the degree of certainty required for a court of law.

Lang and Boyle (82) developed an approach which utilized ion chromatography with suppressed conductivity detection and negative-ion electrospray ionization mass spectrometry (IC-MS). This method facilitated sensitive and reliable detection of the inorganic analytes of interest, as well as the decomposition products of ascorbic acid. However, this approach did not permit the detection of ascorbic acid (as the anionic ascorbate form) because the compound rapidly degraded in the presence of the strongly basic mobile phase. Only the anionic forms of the degradation products threonic acid, 
oxalic acid, and diketogulonic acid could be detected. Also, a second analytical column was required for the analysis of perchlorate. Both intact and post-blast propellant samples were examined.

Because of the limitations of each of the systems described above, a method which would permit the simultaneous analysis of the inorganic oxidizers and organic fuel in ascorbic acid-based propellants was needed. Ideally, this method would be applied to the analysis of both intact and post-blast samples. Given the notoriously complex nature of post-blast samples, it was important that the chosen technique be sensitive, selective, and robust.

\section{B. Development of HPLC-ESI-QToFMS Method}

\section{Selection of Quadrupole Time-of-Flight Mass Spectrometer}

One of the primary benefits of using a quadrupole time-of-flight mass spectrometer (QToFMS) for the analysis of unknown samples is that exact mass measurements are possible. In QToFMS, the mass to charge ratio $(\mathrm{m} / \mathrm{z})$ of each ion is measured to four decimal places, with an error of less than $\pm 5 \mathrm{ppm}$. Such a specific mass measurement means that the identity of the ion can be determined with a high degree of confidence. In contrast, quadrupole and ion trap instruments can only make nominal mass measurements. This means that ions which are separated by less than $1 \mathrm{Da}$ cannot be distinguished. The QToFMS can distinguish low mass ions such as nitrite, nitrate, chlorate, and perchlorate. The nominal and exact masses of these ions, plus ascorbate, are presented in Table 5.1. 
Table 5.1. Nominal and exact masses of analytes of interest. With a QToFMS instrument, accurate mass measurements are collected with an error of less than $\pm 5 \mathrm{ppm}$.

\begin{tabular}{|c|c|c|}
\hline Ion & Nominal Mass $(\mathrm{Da})$ & Exact Mass (Da) \\
\hline Ascorbate & 175 & 175.0242 \\
\hline Nitrite & 46 & 45.9929 \\
\hline Nitrate & 62 & 61.9878 \\
\hline Chlorate & 83 & 82.9536 \\
\hline Perchlorate & 99 & 98.9485 \\
\hline
\end{tabular}

An HPLC unit coupled to the front end of a QToFMS instrument can separate a sample into its components prior to entering the mass spectrometer. By combining these two instruments, information collected from retention time data, accurate mass measurements, and mass spectral fragmentation patterns can be exploited to facilitate identification of the ions of interest. The formation of ion clusters and adducts has been previously noted in the analysis of explosives by HPLC-MS (77) (118). The accurate mass measurements made by the QToFMS can be employed to elucidate the constituents of ion clusters. Both electrospray ionization (ESI) and atmospheric pressure chemical ionization interfaces (APCI) are available for use with HPLC-QToFMS systems. For these reasons, HPLC-QToFMS was chosen as the instrumentation for this project. All method development experiments were performed on a Waters QToFMS Premier instrument at the Bureau of Alcohol, Tobacco, and Firearms Laboratory in Beltsville, MD. Later results involving accurate mass measurements were collected on a Waters QToFMS Micro instrument at the Federal Bureau of Investigation Laboratory in 
Quantico, VA. The experiments were divided in this manner because of issues with equipment availability.

\section{Development of the gradient HPLC method}

Developing a mobile phase to elute perchlorate simultaneously with smaller ions can be difficult (32). Perchlorate is preferentially retained in ion exchange systems, in part because of its large hydrated ionic radius, which impedes the exchange process. Previous work with the Waters IC-Pak Anion HR column and an isocratic isophthalic acid mobile phase resulted in the co-elution of perchlorate with carbonate at thirty minutes (117). Because perchlorate co-eluted with carbonate, perchlorate could not be quantified. Doyle et al. (32) found success in reducing the elution time of perchlorate from the same analytical column by using a complex mobile phase which included an ion pairing agent, and ion exclusion agent, and an organic component. This mobile phase was well suited for conductivity detection. Because one of the goals of this work was to develop a method which permitted the simultaneous analysis of all organic and inorganic anions of interest with detection by mass spectrometry, a new mobile phase was developed for use with the Waters IC-Pak Anion HR column.

A set of protocols outlined in a Waters application note for the analysis of perchlorate was taken as a starting point for the development of the HPLC separation (119). This method involved a Waters IC-Pak Anion HR column with an isocratic mobile phase consisting of 50\% $25 \mathrm{mM}$ ammonium bicarbonate at $\mathrm{pH} 10$ (solvent $\mathrm{A}$ ) and 50\% acetonitrile (solvent B) with ESI-MS detection for the analysis of nitrate, perchlorate, and other inorganic anions. The presence of $50 \%$ acetonitrile in the mobile phase resulted in 
the elution of perchlorate from the column within twelve minutes (119). It should be noted that the use of more than $12 \%$ acetonitrile in the organic portion of the mobile phase was discouraged in the care and use manual for the analytical column (120). Presumably, this statement was included to avoid potential swelling of the stationary phase and subsequent column collapse. However, no adverse effects upon column function or lifespan were noted during the development of the HPLC-ESI-QToFMS method over the course of approximately 400 injections on the column at the ATF laboratory, or another 200 injections on a second column at the FBI laboratory. It should also be noted that the manufacturer recommends this procedure for perchlorate separations.

The use of the mobile phase which was $25 \mathrm{mM}$ ammonium bicarbonate at $\mathrm{pH} 10$ and acetonitrile (50:50) with the Waters IC-Pak Anion HR column resulted in co-elution of chlorate, sulfate, and nitrate at 6.0 minutes, while ascorbate eluted at 2.1 minutes and perchlorate eluted at 12.0 minutes. A series of gradient protocols were examined in an effort to achieve baseline resolution of chlorate, nitrite, and nitrate, while maintaining the separation of ascorbate and perchlorate within thirty minutes of injection. It was determined that use of a $10 \%$ - 50\% acetonitrile gradient resulted in baseline separation of ascorbate, nitrate, nitrite, chlorate, and perchlorate. This gradient program began isocratically with $90 \% 25 \mathrm{mM}$ ammonium bicarbonate at $\mathrm{pH} 10$ and $10 \%$ acetonitrile for four minutes, followed by a one minute ramp to $50 \% 25 \mathrm{mM}$ ammonium bicarbonate at $\mathrm{pH} 10$ and 50\% acetonitrile. This concentration was held for the next twenty-three minutes. At the end of the run, the column was re-equilibrated in $90 \% 25 \mathrm{mM}$ ammonium carbonate at $\mathrm{pH} 10$ and $10 \%$ acetonitrile for seven minutes. Although increasing the 
percentage of acetonitrile in the mobile phase beyond $50 \%$ reduced the elution time of perchlorate, increased levels of background ions in the mass spectra were also observed. Therefore, acetonitrile concentrations greater than 50\% were not employed in the mobile phase. As mentioned earlier, the use of acetonitrile did not appear to have a deleterious effect on column lifetime or function.

The use of $25 \mathrm{mM}$ ammonium bicarbonate at $\mathrm{pH} 10$ in the aqueous component of the mobile phase resulted in the gradual accumulation of particulate material within the mass spectrometer source, leading to a concomitant drop in sensitivity. In order to minimize fouling of the source, the concentration of ammonium bicarbonate in the mobile phase was reduced to $10 \mathrm{mM}$ while the $\mathrm{pH}$ was maintained at 10 . The combination of this mobile phase with the previously developed gradient resulted in a separation which was similar to the $25 \mathrm{mM}$ ammonium bicarbonate mobile phase. Use of this new mobile phase with the QToF Premier resulted in a three-fold improvement in the limit of detection for ascorbate from $15 \mathrm{ppm}$ to $5 \mathrm{ppm}$, and resulted in less accumulation of particulate salt material in the mass spectrometer source region. However, this mobile phase composition lacked sufficient ionic strength to elute sulfate from the analytical column. While other black powder substitutes contain sulfur which generates sulfate in post-blast residues, ascorbic acid-based propellants do not contain sulfur, and the presence of sulfates in post-blast residue would not be expected. Sulfur-containing propellants are best analyzed by IC-MS (82). 


\section{Calibration of QToFMS Micro}

The Waters Quadrupole Time-of-Flight (QToF) mass spectrometer incorporated a LockSpray ${ }^{\mathrm{TM}}$ ion source with electrospray ionization for use in accurate mass measurements. The LockSpray ${ }^{\mathrm{TM}}$ source was mounted onto the standard z-spray interface and provided a low flow capacity electrospray $(<20 \mu \mathrm{l} / \mathrm{min})$ orthogonal to the standard electrospray for introduction of the reference compound. The same electrospray voltage was applied to both the standard electrospray and the reference spray, and the nebulizing gas lines were coupled. The desolvation heater, which was mounted on the standard electrospray, provided heated desolvation gas to the reference spray as well. The LockSpray $^{\mathrm{TM}}$ source included a baffle which admitted spray from either the analyte spray or the reference spray to the sampling cone for acquisition. Spray indexing allowed the analyte and reference data to be acquired into separate data files. The reference solution contained a known compound which had been set as a "lockmass". During an HPLCQToFMS analysis, the reference solution was constantly infused into the reference sprayer. However, the reference spray was only sampled periodically, about once every 10-20 seconds. During the rest of the time, the HPLC eluent was sampled. Data from the reference spray were used to calculate a correction factor for the mass scale, which was then applied to the analyte data to provide accurate mass information (121) (122).

A sodium formate reference solution was used to set the effective length of the flight tube $\left(L_{\text {teff }}\right)$ and to calibrate the instrument across the desired mass range: 45 Da-500 Da. As a result of frequent fluctuations in the temperature of the room where the QToFMS was located, these calibrations were performed at least once per day. The same sodium formate solution was also used as the LockSpray ${ }^{\mathrm{TM}}$ reference solution. This 
solution was chosen as the reference solution because one of its cluster ions of sodium and formate at $m / z 180.9725$ was close to the exact mass of ascorbate (175.0243), and was towards the upper end of the range of masses which were being analyzed. When the sodium formate solution was used as the LockSpray ${ }^{\mathrm{TM}}$ reference solution, an accuracy range of $\pm 5 \mathrm{ppm}$ or better for all target analytes was achieved.

\section{Analysis of standards and method validation}

Standard solutions of ascorbic acid, nitrite, nitrate, chlorate, and perchlorate were analyzed by HPLC-ESI-QToFMS Micro at the following concentrations: $400 \mathrm{ppm}$ ascorbic acid, 500 ppm nitrite, 25 ppm chlorate, 25 ppm nitrate, and 2 ppm perchlorate. Data were acquired in continuum mode and afterwards processed by the MassLynx software (Waters Corporation) to perform data centering and lock mass correction. To obtain accurate mass measurements, at least 15 scans were combined. The mass data were centered using peak areas at $80 \%$ of the centroid top. Figure 5.1 a displays the total ion chromatogram and extracted ion chromatograms for these data, while the mass spectra for these anions are presented in Figures 5.1b - 5.1f. The extracted ion chromatograms display an increase in selectivity versus the total ion current. Extracted ion chromatograms are essential for the detection of the target anions at trace levels in real samples. The identities of the peaks at $m / z 175.0249,44.9928,82.9536,61.9876$, 98.9485 were confirmed by use of the elemental composition calculator function of the MassLynx software. The search function of the MassLynx software was employed to match the experimentally determined mass to the most likely candidate based on a set of user defined criteria, including the expected number and range of elements, the upper 
limit on the number of double-bond-equivalent (DBE) values, and the acceptable error. If more than one match was possible, then each candidate was ranked based on calculated mass and isotopic profiles. Element composition searches typically yielded a limited number of molecule options, in contrast to nominal molecular mass searches which can return overwhelming numbers of molecule options. The MassLynx software correctly identified the ions at $m / z 175.0249,44.9928,82.9536,61.9876,98.9485$ as belonging to ascorbate, nitrite, chlorate, nitrate, and perchlorate, respectively. All measured masses were within $\pm 5 \mathrm{ppm}$ of the calculated mass for each ion as summarized in Table 5.2. It should be noted that the mass accuracy for a given ion is dependent upon the intensity for the signal of that ion. Too few ions lead to poor ion statistics, while too many ions lead to detector saturation. Both of these outcomes result in greater variation in the standard deviation of repeat measurement and will adversely affect the calculation of the error. The retention time of each anion is also presented in Table 5.2.

Figure 5.1a shows the extracted ion chromatogram of nitrite. Nitrite has a molecular ion at $\mathrm{m} / \mathrm{z} 46$ and contained two peaks: one at 9.14 minutes and the other at 13.28 minutes. The first peak corresponded to the retention time for nitrite, while the second peak corresponded to the retention time for nitrate, indicating that nitrate decomposes or fragments to nitrite in the source of the mass spectrometer.

The peak at 9.97 minutes in the total ion chromatogram in Figure 5.1a does not correspond to any of the anions in the standard mix. This peak appeared at the same retention time in all of the samples which were analyzed, including the blank samples. The size of this peak was more pronounced when the percentage of acetonitrile in the mobile phase was $65 \%$, and absent when the percentage of acetonitrile in the mobile 
phase was limited to $40 \%$, thereby indicating a possible system peak resulting from the acetonitrile mobile phase.

Figure 5.1. Analysis of a standard solution of ascorbic acid (400 ppm), nitrite (500ppm), chlorate (25 ppm), nitrate (25 ppm), and perchlorate (2 ppm) by HPLC-ESI-QToFMS. For conditions, see text. (a) Total ion chromatogram and extracted ion chromatograms for ascorbate, nitrite, chlorate, nitrate, and perchlorate (b) mass spectrum of ascorbate (c) mass spectrum of nitrite (d) mass spectrum of chlorate (e) mass spectrum of nitrate (f) mass spectrum of perchlorate. Background-subtracted mass spectra were collected with subsequent accurate mass measurement using the lock mass provided by the second ESI probe.

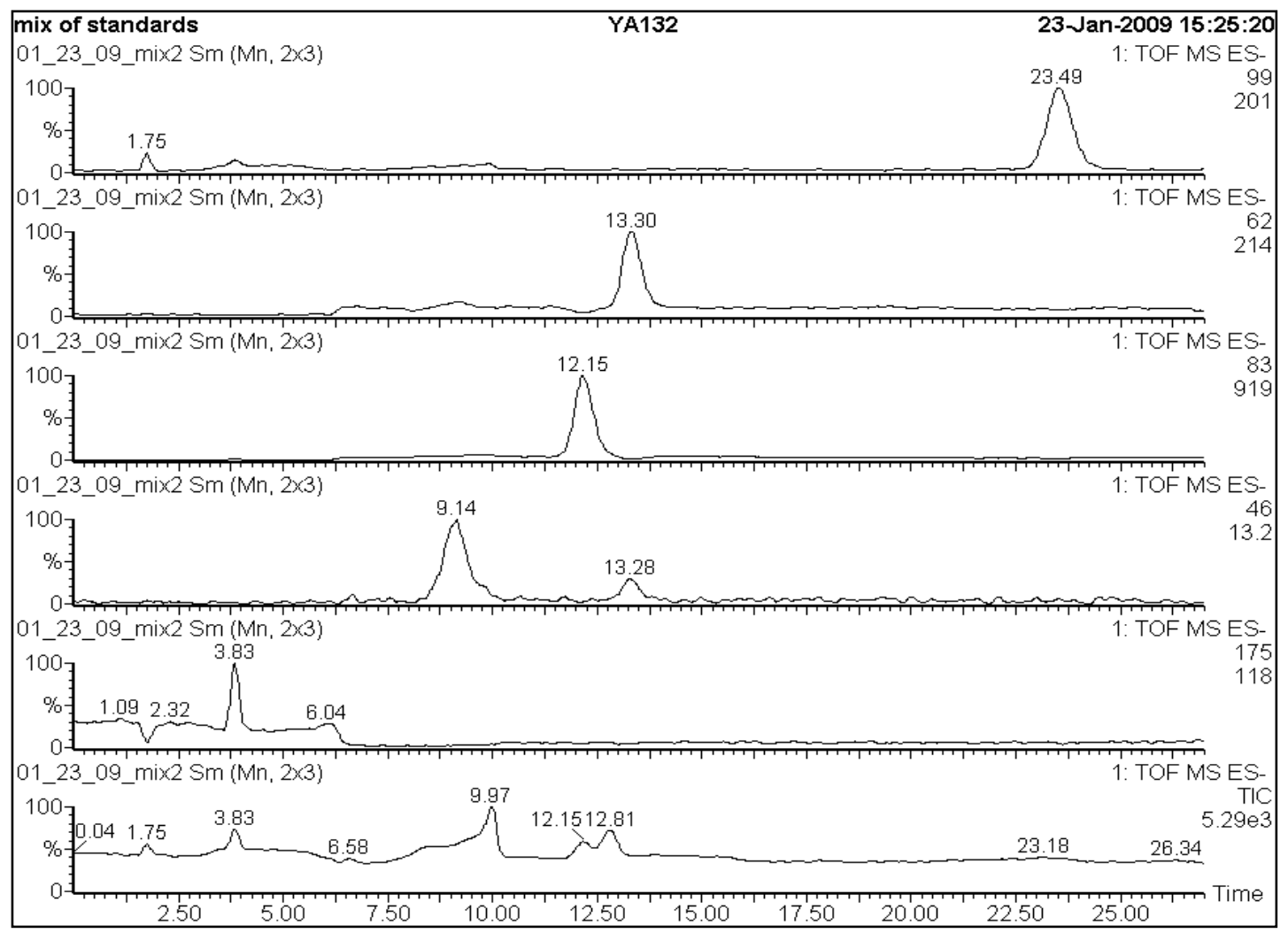



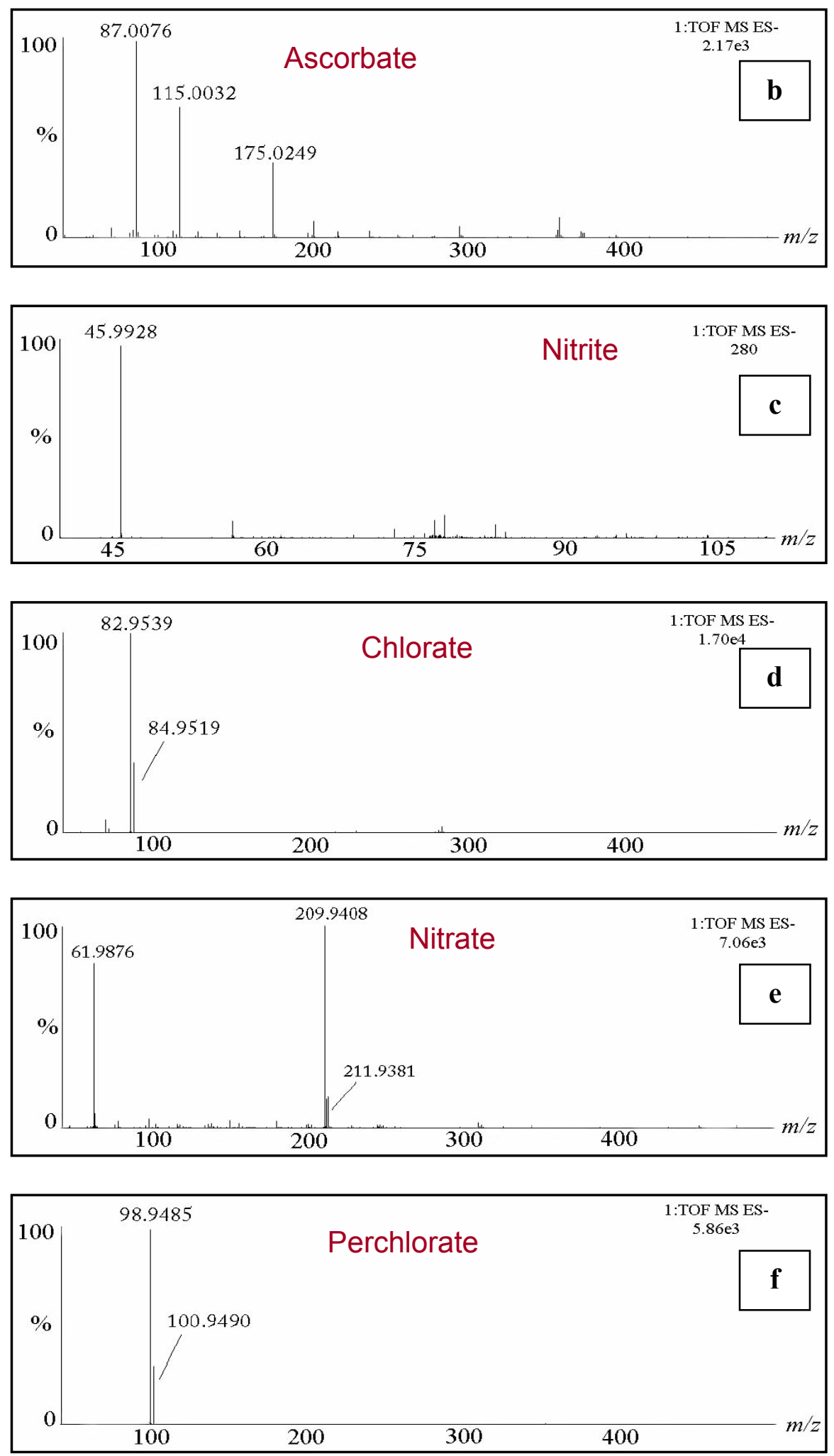
Table 5.2. Accurate mass data for the anion standards by HPLC-ESI-QToFMS.

\begin{tabular}{|c|c|c|c|c|c|}
\hline Component & Rt (min) & Calculated $\mathrm{m} / z$ & Measured $\mathrm{m} / \mathrm{z}$ & $\pm \mathrm{mDa}$ & \pm ppm \\
\hline Ascorbate & 3.83 & 175.0243 & 175.0249 & 0.6 & 3.4 \\
\hline Nitrite & 9.14 & 45.9929 & 45.9928 & -0.1 & -2.2 \\
\hline Chlorate & 12.15 & 82.9536 & 82.9539 & 0.3 & 3.6 \\
\hline Nitrate & 13.30 & 61.9878 & 61.9876 & -0.2 & -3.2 \\
\hline Perchlorate & 23.49 & 98.9485 & 98.9485 & 0.0 & 0.0 \\
\hline
\end{tabular}

Table 5.3. Figures of merit for anion standards by HPLC-ESI-QToFMS. Ten replicate injections of a standard mixture of ascorbic acid, nitrite, chlorate, nitrate, and perchlorate were performed. The LOD is the concentration of the analyte which gave a signal three times the standard deviation of the blank.

\begin{tabular}{|c|c|c|c|c|c|c|}
\hline Anion & Formula & Ave Rt (min) & Stnd Dev & $\% R S D$ & LOD $(\mu \mathrm{g} / \mathrm{mL})$ & LOD $(\mu \mathrm{M})$ \\
\hline Ascorbate & $\mathrm{C}_{6} \mathrm{H}_{5} \mathrm{O}_{6}^{-}$ & 3.81 & 0.06 & 1.6 & 69 & 390 \\
\hline Nitrite & $\mathrm{NO}_{2}^{-}$ & 9.02 & 0.22 & 2.5 & 189 & 4500 \\
\hline Chlorate & $\mathrm{ClO}_{3}^{-}$ & 12.06 & 0.20 & 1.6 & 1.0 & 12 \\
\hline Nitrate & $\mathrm{NO}_{3}^{-}$ & 13.09 & 0.22 & 1.7 & 9.0 & 145 \\
\hline Perchlorate & $\mathrm{ClO}_{4}^{-}$ & 22.99 & 0.43 & 1.9 & 0.04 & 0.4 \\
\hline
\end{tabular}

Table 5.3 presents data from a series of replicate injections of a standard mix of nitrite, nitrate, chlorate, perchlorate, and ascorbate that were performed to test the system precision. All of the target analytes eluted from the column within twenty-five minutes of injection. The $\%$ RSD (relative standard deviation) of the retention times ranged from 
$1.6 \%$ for ascorbate, to $2.5 \%$ for nitrite; the retention time of all the target analytes was highly reproducible.

The limit of detection (LOD) is defined as the concentration which gives an instrument signal which is significantly different from the blank or background signal. In practical terms, this can be defined as the analyte concentration giving a signal equal to the blank signal $\left(\mathrm{y}_{\mathrm{B}}\right)$ plus three standard deviations of the blank, $\mathrm{s}_{\mathrm{B}}$ (see below) (116).

$$
\mathrm{y}-\mathrm{y}_{\mathrm{B}}=3 \mathrm{~s}_{\mathrm{B}}
$$

To calculate the limit of detection using this format, ten replicate injections were performed for each anion standard at a concentration which was very near to the theorized instrument detection limit. The average and standard deviation of these measurements were determined, and this information was substituted into the above equation. The resulting y-value was converted to a concentration value; this value corresponded to the limit of detection in terms of sample concentration. These data are listed in Table 5.3. Values ranged from $0.04 \mathrm{ppm}$ for perchlorate, to $189 \mathrm{ppm}$ for nitrite. Although perchlorate, chlorate, and nitrate were readily detected on both instruments, the ability of both of the QToFMS instruments to detect smaller ions was reduced as the mass-to-charge ratio of the analytes decreased below $100 \mathrm{Da}$. It should be noted that neither the QToFMS Premier nor the QToFMS Micro systems were recommended by the manufacturer for work with analytes of mass $100 \mathrm{Da}$ or less as these instruments do not readily detect such small, fast-moving ions. Chloride could not be detected even at 1000 ppm, despite repeated experiments by direct injection and with the HPLC interface. 


\section{Analysis of Nitrate Clusters}

The analysis of the nitrate standard yielded an interesting phenomenon that was also observed in the spectra of each of the nitrate-containing propellant samples. The chromatographic peak for nitrate at 13.30 min yielded a molecular anion at $\mathrm{m} / \mathrm{z} 209.9408$ in addition to the nitrate peak at $m / z 61.9876$ (see Figure 5.1e). In order to determine the nature of the peak at $m / z$ 209.9408, an MS/MS experiment was performed by fragmenting this anion at a collision cell energy of $15 \mathrm{eV}$. The results of this MS/MS experiment are presented in Figures $5.2 \mathrm{a}-\mathrm{c}$. and all measured masses are within $\pm 5 \mathrm{ppm}$ of the calculated mass for each ion as shown in Table 5.4.

Figures 5.2a-c demonstrates that the anion at $\mathrm{m} / \mathrm{z} 209.9409$ fragments to produce anions at $\mathrm{m} / z$ 61.9881. This result indicated that the ion at $\mathrm{m} / \mathrm{z} 209.9409$ was actually a cluster which included at least one nitrate ion. The accurate mass calculator of the MassLynx software suggested that the closest match within $\pm 5 \mathrm{ppm}$ was at $\mathrm{m} / z$ 209.9401. This mass corresponded to the following ion cluster:

$$
\left[\mathrm{NO}_{3}{ }^{-} \mathrm{NO}_{3}^{-} \mathrm{NO}_{2}^{-} \mathrm{H}^{+} \mathrm{K}^{+}\right]
$$

This cluster has a net negative charge, so it is reasonable to think it would be detected in negative ESI mode. The species with potassium should contain a significant $\mathrm{M}+2$ isotope, since the abundance of ${ }^{41} \mathrm{~K}$ is $6.7 \%$. This $\mathrm{M}+2$ peak is clearly evident in Figure 5.2b. The theoretical model generated from the MassLynx software and the actual acquired data for the cluster are presented in Figures 5.3a-b. The measured $m / z$ of the nitrate anion was 61.9881 , which was within $\pm 0.3 \mathrm{mDa}( \pm 4.8 \mathrm{ppm})$ of the calculated value. The measured $\mathrm{m} / \mathrm{z}$ of the cluster was 209.9409 , which was within $\pm 0.8 \mathrm{mDa}( \pm 3.8$ ppm) of the calculated value. Based upon the isotopic profile of the cluster, and the 
results of the MS/MS experiment, it is likely that the $\mathrm{m} / \mathrm{z} 209.9409$ peak corresponds to the $\left[\mathrm{NO}_{3}{ }^{-} \mathrm{NO}_{3}{ }^{-} \mathrm{NO}_{2}{ }^{-} \mathrm{H}^{+} \mathrm{K}^{+}\right]$cluster. The tendency of nitrate to form clusters in ESI-mass spectrometry has been previously noted (123) (124) (125). However, in these studies, the nitrate clusters assumed the following formula: $\left[\left(\mathrm{KNO}_{3}\right)_{\mathrm{n}} \mathrm{NO}_{3}{ }^{-}\right]$, where $\mathrm{n} \geq 1$.

Figure 5.2. Analysis of the unknown ion at $m / z 209.9409$ by HPLC-ESI-QToFMS-MS with collision energy $15 \mathrm{eV}$. For conditions, see text. (a) Total ion chromatogram, extracted ion chromatograms for nitrate and $m / z$ 209.9409, and extracted ion chromatogram for $m / z 209.9409$ from MS/MS experiment in ascending order (b) mass spectrum of nitrate and $\mathrm{m} / \mathrm{z} 209.9409$ prior to fragmentation (c) mass spectrum of $\mathrm{m} / \mathrm{z}$ 209.9409 following MS-MS experiment. Fragmentation of the cluster at $m / z 209.9409$ yielded nitrate ions.

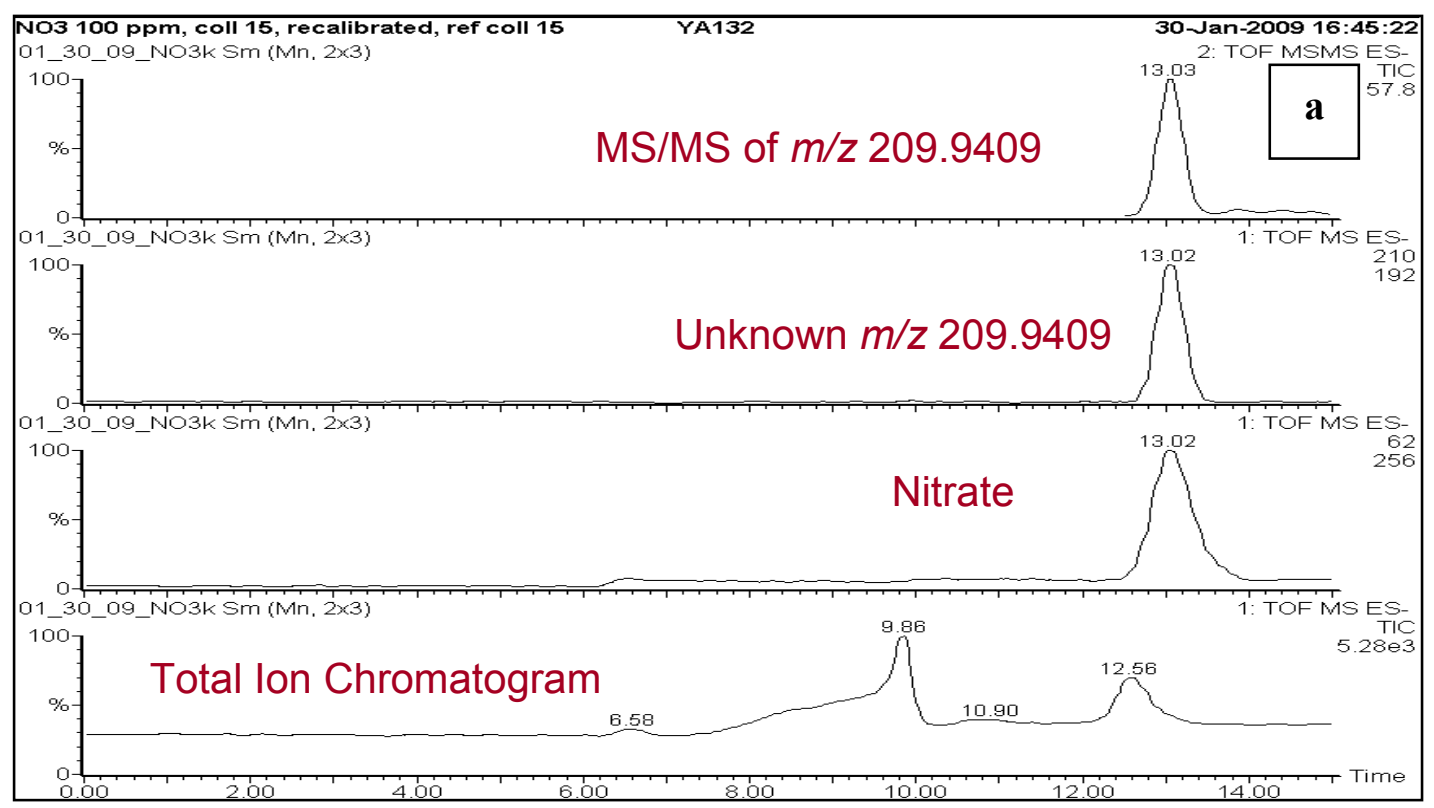



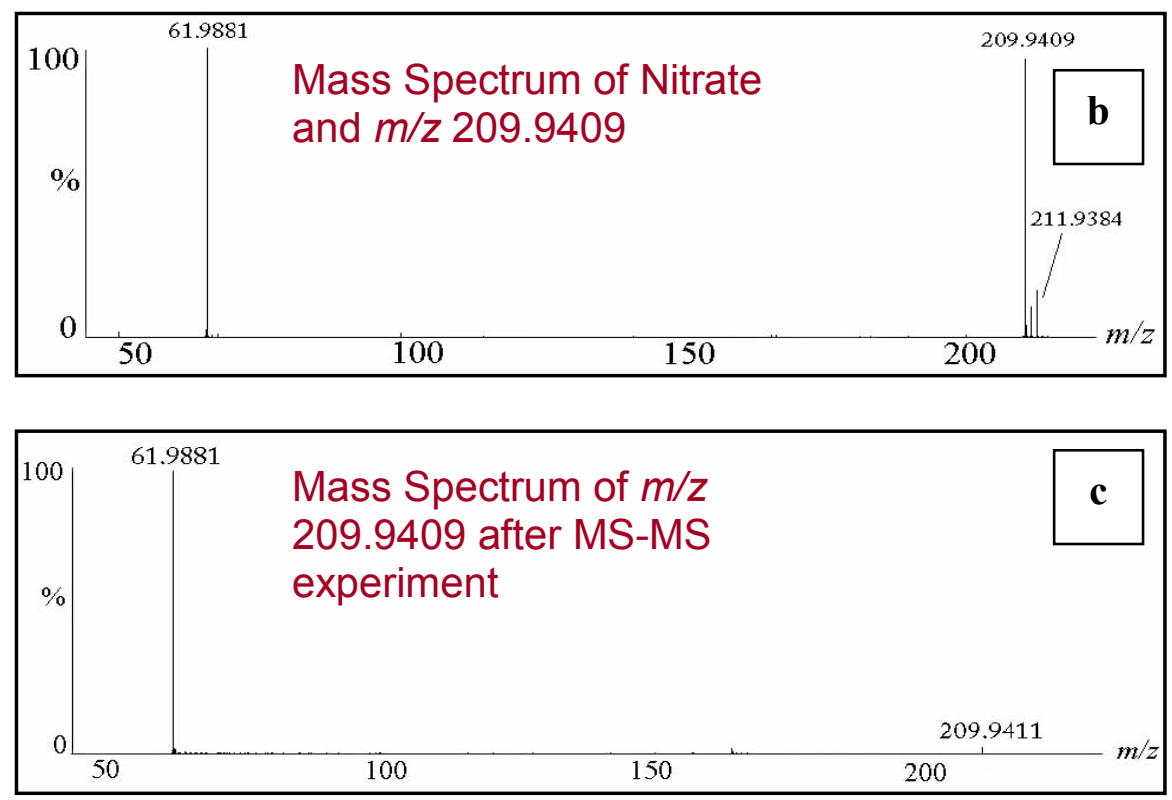

Table 5.4. Accurate mass data for nitrate and nitrate cluster.

\begin{tabular}{|c|c|c|c|c|}
\hline Component & Calculated $\mathrm{m} / \mathrm{z}$ & Measured $\mathrm{m} / \mathrm{z}$ & $\pm m \mathrm{ma}$ & $\pm p p m$ \\
\hline Nitrate & 61.9878 & 61.9881 & 0.3 & 4.8 \\
\hline Nitrate Cluster & 209.9401 & 209.9409 & 0.8 & 3.8 \\
\hline
\end{tabular}

Figure 5.3. Isotopic distribution of $\left[\mathrm{NO}_{3}{ }^{-} \mathrm{NO}_{3}{ }^{-} \mathrm{NO}_{2}{ }^{-} \mathrm{H}^{+} \mathrm{K}^{+}\right]$cluster at $m / z 209.9401$ (a) theoretical model (b) acquired data.
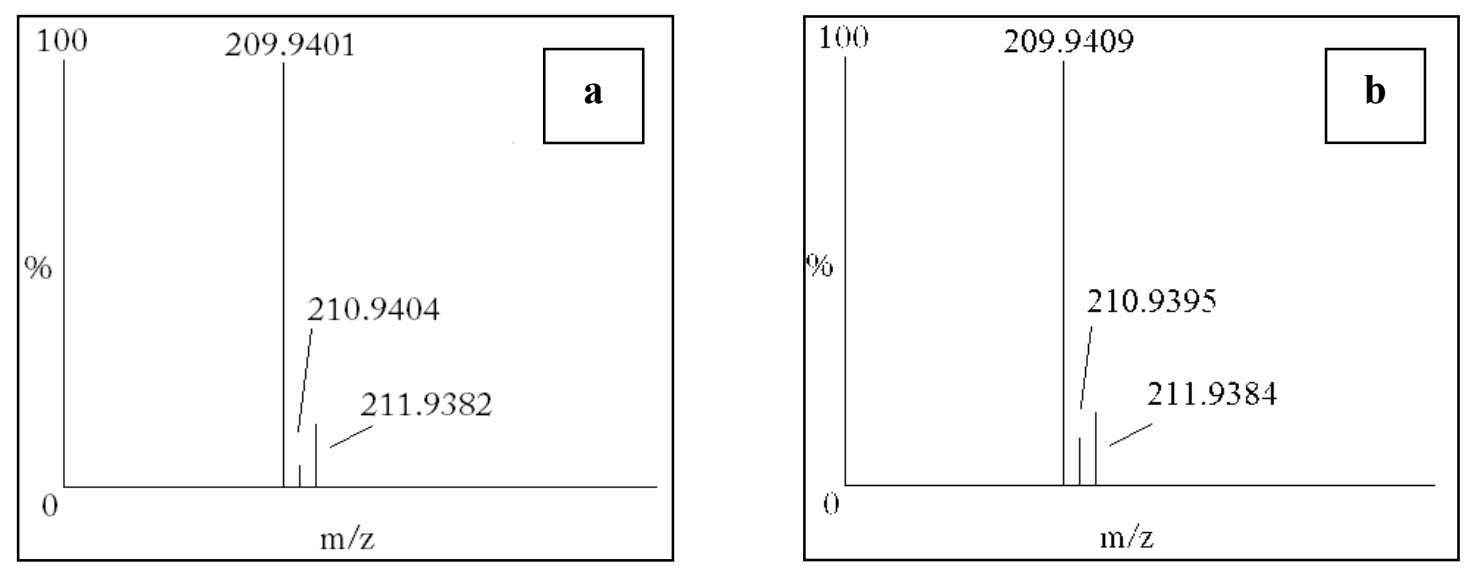


\section{Ascorbic Acid Fragmentation Patterns}

Depending upon the conditions, ascorbic acid can form a variety of degradation products (111) (112). In solution, ascorbic acid first oxidizes reversibly to dehydroascorbic acid, which then degrades irreversibly to 2,3-diketogulonic acid. Goodpaster and Keto illustrated the reaction pathway listed in Figure 5.4, whereby the 2,3-diketogulonic acid further degrades to form threonic acid and oxalic acid (89). A more detailed degradation pathway for ascorbic acid was offered by Cioffi et al. (115). In this pathway, the dehydroascorbic acid rapidly undergoes re-hydration, followed by spontaneous hydrolysis at the lactonic ring. This results in the formation of the diketogulonate anion in its monohydrated and/or dehydrated forms.

However, under the vacuum conditions of the QToFMS instrument, a different fragmentation pattern was observed for the ascorbate anion. As seen in Figure 5.5, analysis of ascorbic acid by HPLC-ESI-QToFMS resulted in three MS peaks at $\mathrm{m} / \mathrm{z}$ 175.0242, 115.0026, and 87.0083. These three $\mathrm{m} / \mathrm{z}$ peaks of ascorbic acid were noted in both direct injection experiments and samples introduced to the mass spectrometer via HPLC inlet. The identities of the anions at $\mathrm{m} / \mathrm{z} 115.0026$ and $\mathrm{m} / \mathrm{z} 87.0083$ were confirmed to be $\mathrm{C}_{4} \mathrm{H}_{3} \mathrm{O}_{4}$ and $\mathrm{C}_{3} \mathrm{H}_{3} \mathrm{O}_{3}$, respectively, using the elemental composition calculator function of the MassLynx software. As Table 5.5 shows, the error of measured masses of all three anions is less than \pm 5 ppm from their calculated masses. 
Figure 5.4. Degradation process of ascorbic acid to yield 2,3-diketogulonic acid, threonic acid, and oxalic acid (89).<smiles>O=C1OC(C(O)CO)C(O)=C1O</smiles>

Ascorbic Acid $\mathrm{MW}=176$

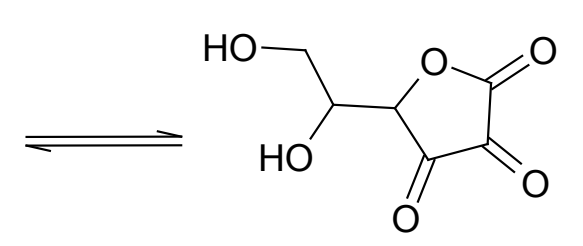

Dehydroascorbic Acid $\mathrm{MW}=174$<smiles>O=C(O)C(=O)C(=O)C(O)C(O)CO</smiles>

2,3-Diketogulonic Acid $\mathrm{MW}=192$<smiles>O=C(O)C(O)C(O)CO</smiles>

Threonic Acid, 2,3,4-Trihydroxybutanoic Acid $\mathrm{MW}=136$<smiles>O=C(O)C(=O)O</smiles>

Oxalic Acid

1,2-Ethanedioic Acid $\mathrm{MW}=90$ 
Figure 5.5. Mass spectrum from the analysis of ascorbate by HPLC-ESI-QToFMS. In addition to the ascorbate peak at $m / z$ 175.0242, two fragment peaks are present at $m / z$ 115.0026 and $m / z 87.0083$.

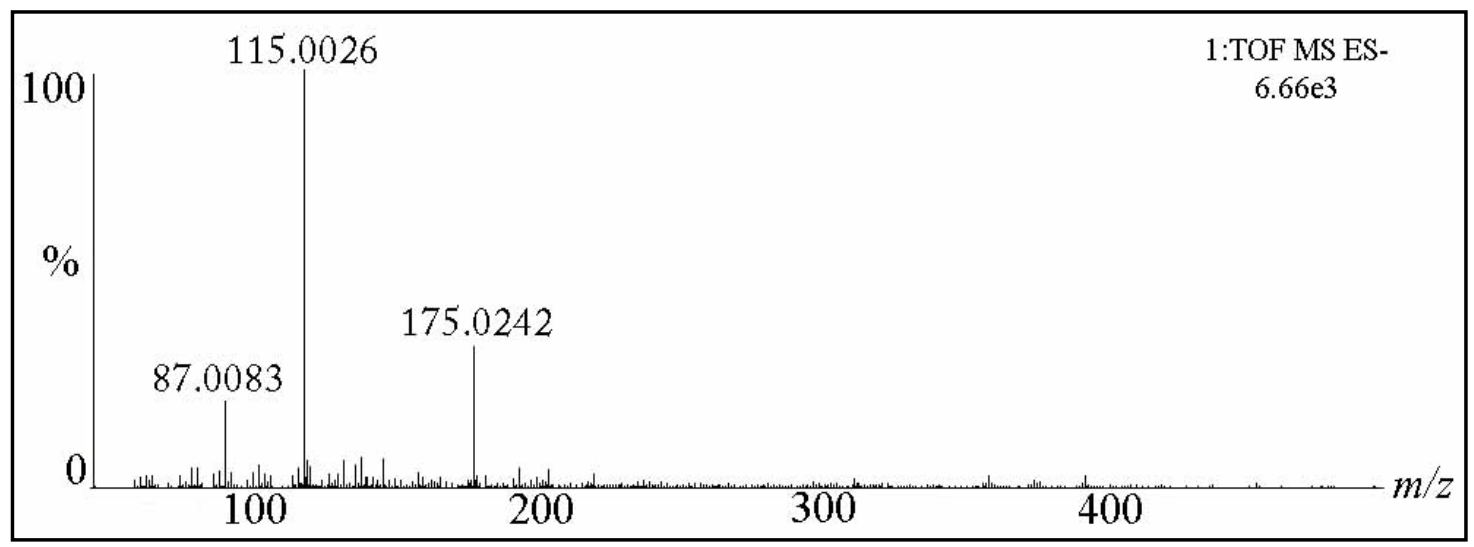

Table 5.5. Elemental composition report from MassLynx software regarding identification of ascorbate and the fragment anions.

\begin{tabular}{|c|c|c|c|c|c|}
\hline Measured & Relative & Calculated & Error & Error & Formula \\
Mass & Abundance & Mass & $\pm m D a$ & $\pm p p m$ & \\
\hline 87.0083 & 20.34 & 87.0082 & 0.1 & 1.1 & $\mathrm{C}_{3} \mathrm{H}_{3} \mathrm{O}_{3}$ \\
\hline 115.0026 & 100.00 & 115.0031 & -0.5 & -4.3 & $\mathrm{C}_{4} \mathrm{H}_{3} \mathrm{O}_{4}$ \\
\hline 175.0242 & 33.59 & 175.0243 & -0.1 & -0.6 & $\mathrm{C}_{6} \mathrm{H}_{7} \mathrm{O}_{6}$ \\
\hline
\end{tabular}

This pattern of peaks in the mass spectrum of ascorbate is consistent with the previous work done on the analysis of ascorbic acid-based propellants by LC-ESI-MS (80) (90). These two LC-ES-IMS studies reported that the molecular anion of ascorbic acid, ascorbate, was detected at $m / z 175$ along with two fragment anions at $m / z 87$ and $m / z 115$. Neither of these studies employed a mass spectrometer capable of performing 
accurate mass measurements. It was theorized that the fragment at $m / z 115$ was formed through the loss of $\mathrm{O}=\mathrm{CH}_{2} \mathrm{CH}_{2}-\mathrm{O}^{-}$from $\mathrm{m} / \mathrm{z} 175$, and the fragment at $\mathrm{m} / \mathrm{z} 87$ was formed through the loss of $\mathrm{CO}$ from $m / z 115(80)$. Based on the results of the current experiment, a fragmentation pathway can be proposed whereby the ascorbate anion at $\mathrm{m} / \mathrm{z} 175.0242$ lost 60.0216 Da to form the anion at $m / z$ 115.0026, which subsequently lost 27.9943 Da to form the anion at $m / z$ 87.0083. This pathway is outlined in Figure 5.6. Additional degradation products of ascorbic acid, $m / z 191$ (mono-hydrated dehydroascorbic acid), 209 (mono-hydrated diketogulonate), 227 (dihydrated diketogulonate), 89 (oxalate), or 135 (threonate) were note observed during the analysis of intact samples of ascorbic acid.

Figure 5.6. Alternative degradation pathway for ascorbate to yield fragment ions at $\mathrm{m} / \mathrm{z}$ 115.0026 and $m / z 87.0083$.
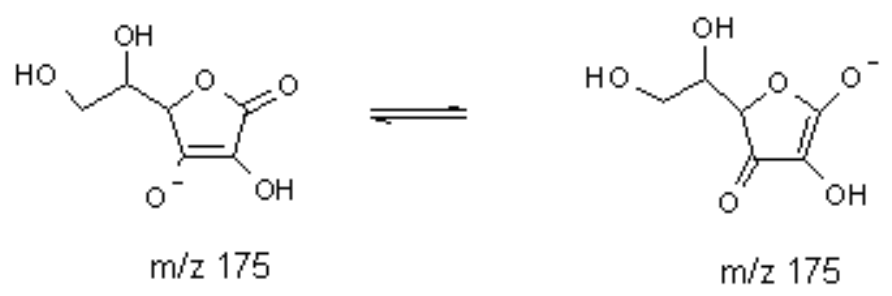

$\mathrm{m} / \mathrm{z} 175$

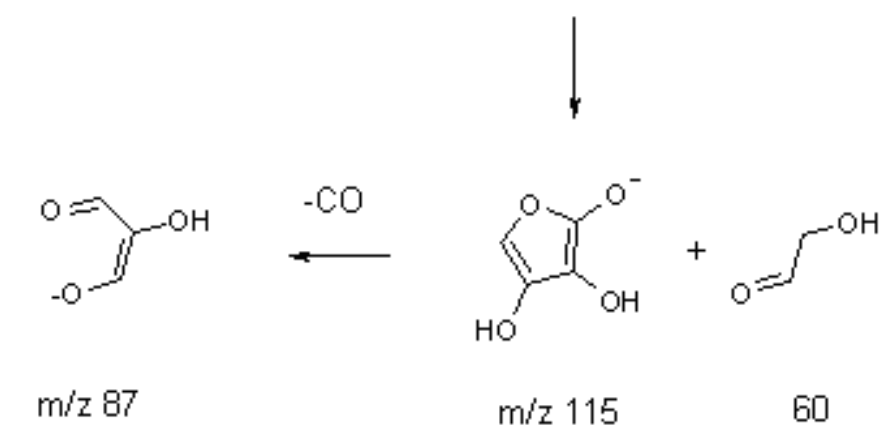




\section{Results of Sample Analysis}

Six brands of intact propellant samples, including American Pioneer, Black Mag3, Black Canyon, Clean Shot, Goex Pinnacle Replica, and Jim Shockey’s Gold were analyzed using the optimized gradient HPLC-ESI-QToFMS method. Table 5.6 summarizes the composition information of each propellant. Five of the six propellants contain ascorbic acid as the fuel source and potassium nitrate $\left(\mathrm{KNO}_{3}\right)$ and potassium perchlorate $\left(\mathrm{KClO}_{4}\right)$ as the oxidizers. In contrast, Black Canyon contains an ascorbic acid fuel source with only potassium nitrate as the oxidizer compound.

Table 5.6. Composition of black powder substitutes. All six propellants contain an ascorbic acid fuel source and a nitrate oxidizer. Only Black Canyon does not contain a perchlorate oxidizer (34) (89).

\begin{tabular}{|c|c|}
\hline Propellant & Composition \\
\hline American Pioneer & $\mathrm{AA}, \mathrm{KNO}_{3}, \mathrm{KClO}_{4}$ \\
\hline Black Canyon & $\mathrm{AA}, \mathrm{KNO}_{3}$ \\
\hline Black Mag3 & $\mathrm{AA}, \mathrm{KNO}_{3}, \mathrm{KNO}_{4}$ \\
\hline Clean Shot & $\mathrm{AA}, \mathrm{KNO}_{3}, \mathrm{KNO}_{4}$ \\
\hline Goex Pinnacle Replica & $\mathrm{AA}, \mathrm{KNO}_{3}, \mathrm{KNO}_{4}$ \\
\hline Jim Shockey's Gold & $\mathrm{AA}, \mathrm{KNO}_{3}, \mathrm{KNO}_{4}$ \\
\hline
\end{tabular}


Figure 5.7a-e displays the data collected for an intact sample of American Pioneer. These results were typical for all of the intact propellants with the exception of Black Canyon. Figure 5.7a displays the total ion chromatogram and the extracted anion profiles of ascorbate, chlorate, nitrate, and perchlorate. The corresponding mass spectra of each anion are presented in Figures 5.7b-e. The accurate mass data for this sample and the retention time for each analyte are presented in Table 5.7.

As figure 5.7a shows, ascorbate elutes at 3.85 minutes, nitrate at 13.13 minutes, and perchlorate at 22.77 minutes. The chromatographic peak for ascorbate (see Figure 5.7b) contains all three characteristic ions: the molecular anion at $\mathrm{m} / \mathrm{z} 175.0242$, and the fragment anions at $\mathrm{m} / \mathrm{z} 115.0026$ and $\mathrm{m} / \mathrm{z}$ 87.0081. Nitrate has its molecular anion at $\mathrm{m} / \mathrm{z}$ 61.9881, and the cluster anion at $m / z 209.9402$ as shown in Figure 5.7d. This ion cluster appears to be concentration dependent, as the ratio of $\mathrm{m} / \mathrm{z} 210$ is higher on the more intense spectrum. In Figure 5.7e, perchlorate has a molecular anion at $m / z$ 98.9488, and an isotopic peak at $\mathrm{m} / \mathrm{z}$ 100.9467. In addition to the expected anions (ascorbate, nitrate, and perchlorate), chlorate is also detected in the American Pioneer sample at the retention time 12.17 minutes (see Figure 5.7a). The $m / z 82.9536$ peak in Figure 5.7c is the molecular anion of chlorate, and $m / z 84.9508$ is an isotopic peak. It is unknown whether the chlorate in this sample is a degradation product of perchlorate (as a result of poor storage conditions), or a deliberate addition on the part of the manufacturer to improve the burning rate of the propellant. 
Figure 5.7. Analysis of intact grains of American Pioneer by HPLC-ESI-QToFMS (a) total ion chromatogram and extracted ion chromatograms for ascorbate, chlorate, nitrate, and perchlorate (b) mass spectrum of ascorbate (c) mass spectrum of chlorate (d) mass spectrum of nitrate (e) mass spectrum of perchlorate.

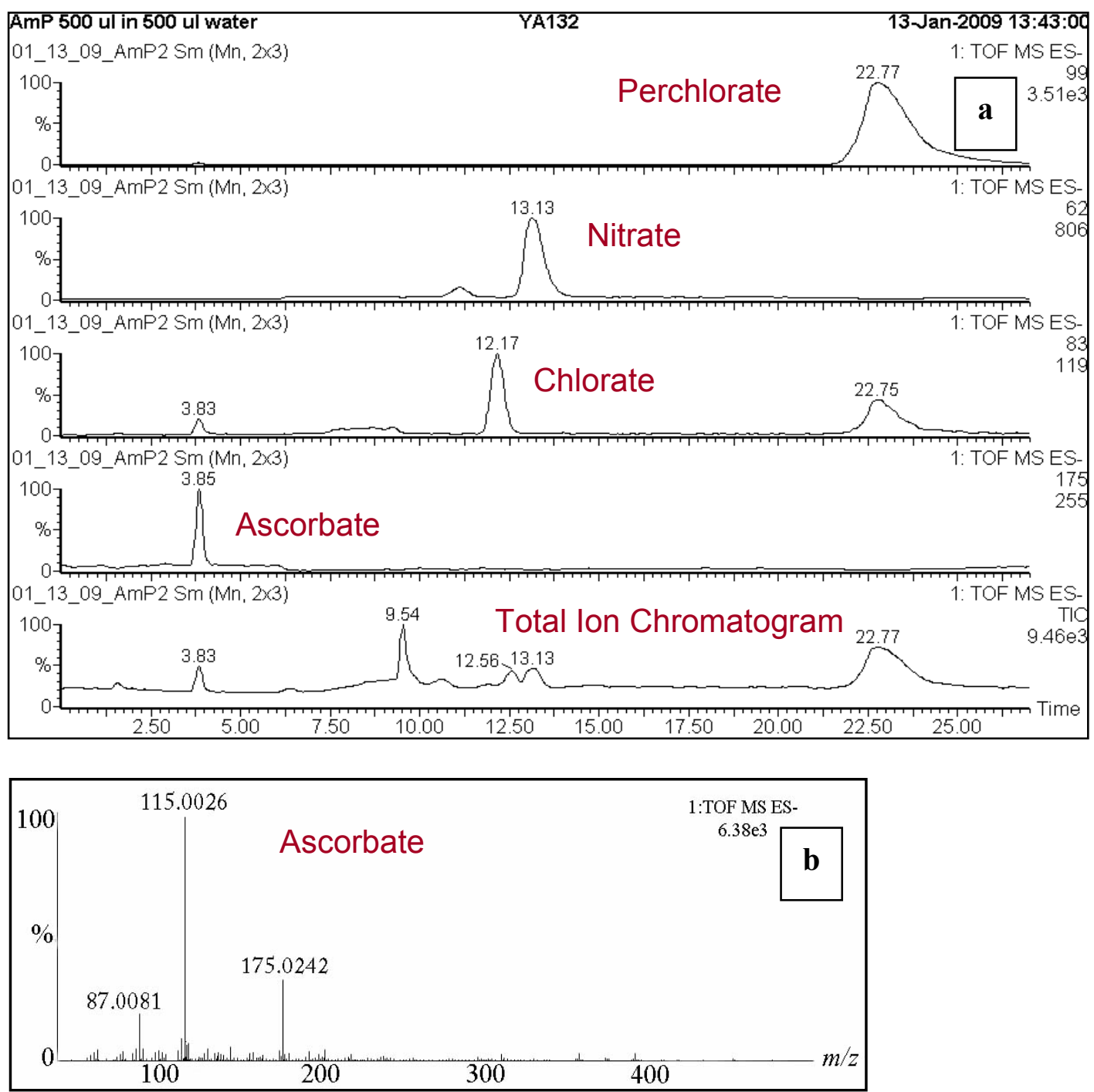



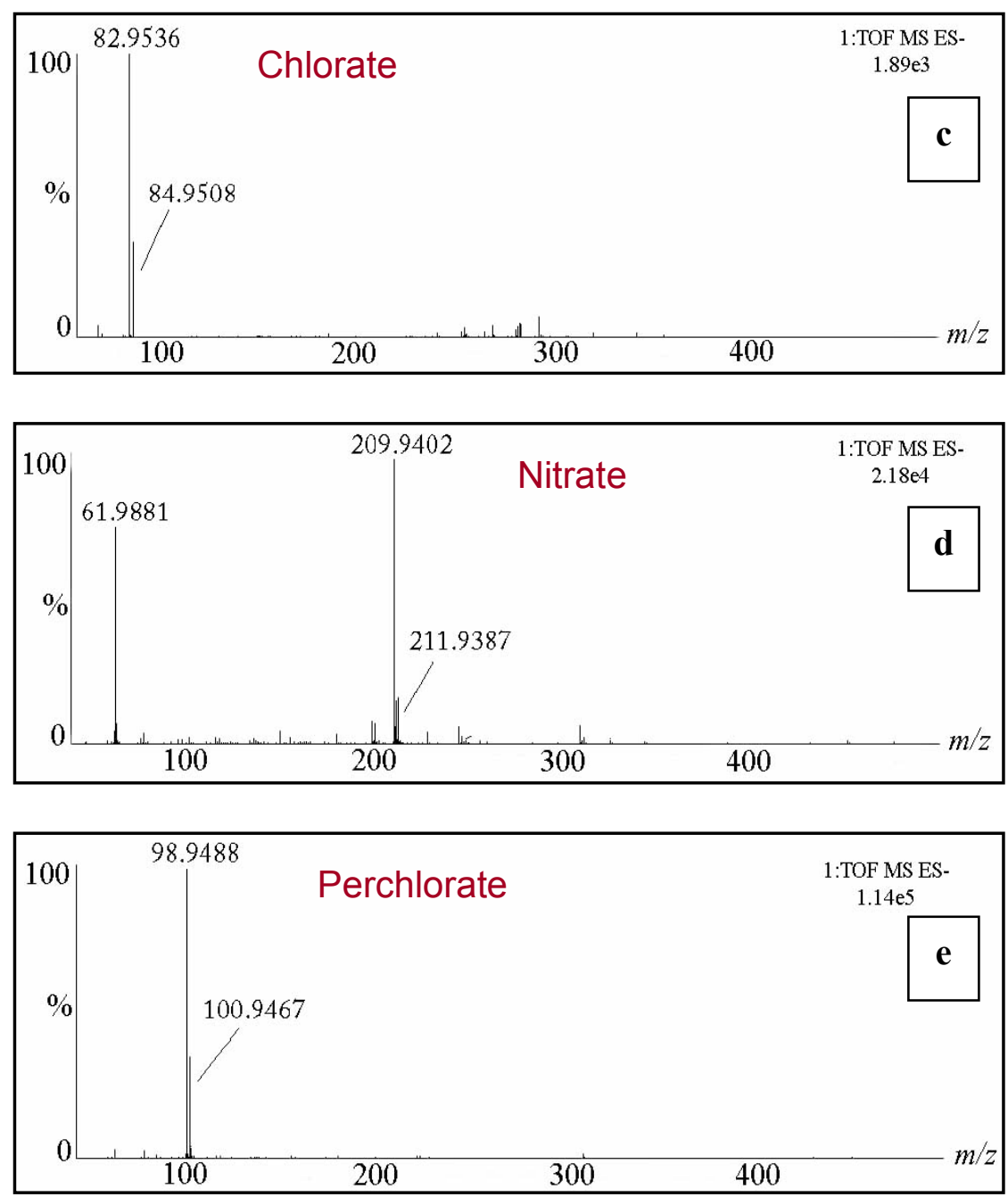

Table 5.7. Accurate mass data for the anions present in intact grains of American

Pioneer.

\begin{tabular}{|c|c|c|c|c|c|}
\hline Component & $R t$ (min) & Calculated $\mathrm{m} / \mathrm{z}$ & Measured $\mathrm{m} / \mathrm{z}$ & $\pm m D a$ & $\pm p p m$ \\
\hline Ascorbate & 3.85 & 175.0243 & 175.0242 & -0.1 & -0.6 \\
\hline Chlorate & 12.17 & 82.9536 & 82.9536 & 0.0 & 0.0 \\
\hline Nitrate & 13.13 & 61.9878 & 61.9881 & 0.3 & 4.8 \\
\hline Perchlorate & 22.77 & 98.9485 & 98.9488 & 0.3 & 3.0 \\
\hline
\end{tabular}


Figure 5.8. Analysis of intact grains of Goex Pinnacle Replica by HPLC-ESI-QToFMS (a) total ion chromatogram and extracted ion chromatograms for ascorbate, nitrate, and perchlorate (b) mass spectrum of ascorbate (c) mass spectrum of nitrate (d) mass spectrum of perchlorate.

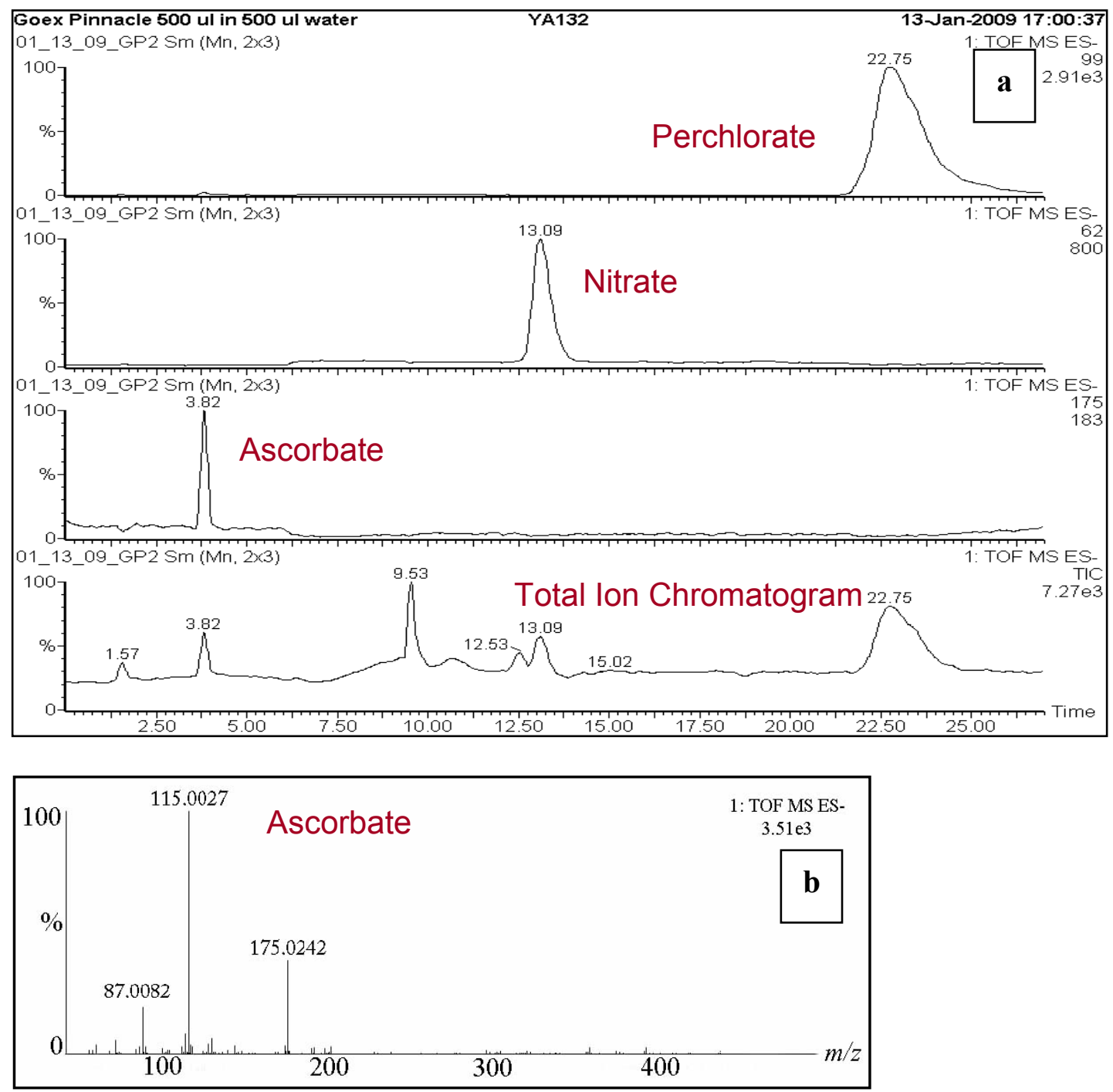



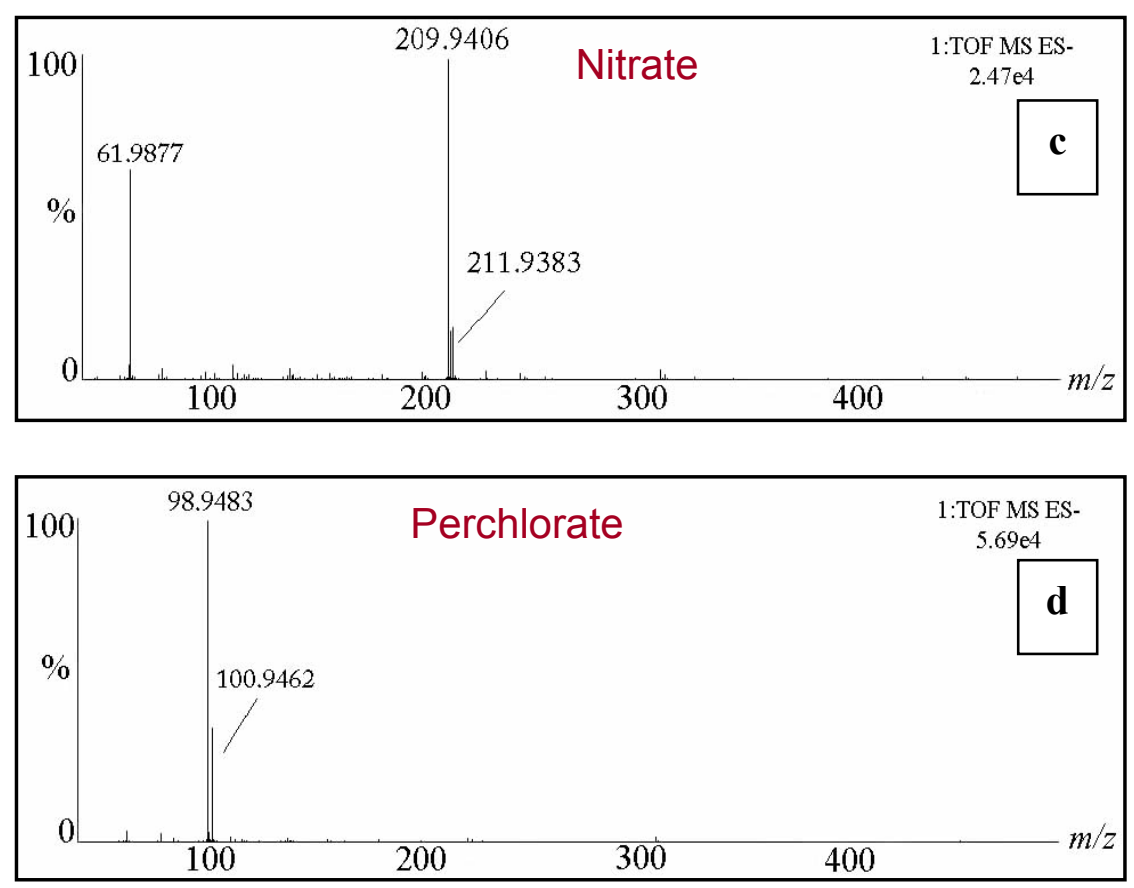

Table 5.8. Accurate mass data for the anions present in intact grains of Goex Pinnacle Replica.

\begin{tabular}{|c|c|c|c|c|c|}
\hline Component & Rt (min) & Calculated $m / z$ & Measured $m / z$ & $\pm m D a$ & $\pm p p m$ \\
\hline Ascorbate & 3.82 & 175.0243 & 175.0242 & -0.1 & -0.6 \\
\hline Nitrate & 13.09 & 61.9878 & 61.9877 & -0.1 & -1.6 \\
\hline Perchlorate & 22.75 & 98.9485 & 98.9483 & -0.2 & -2.0 \\
\hline
\end{tabular}

Figure 5.8a-e displays data collected for an intact sample of Goex Pinnacle Replica. Figure 5.8a displays the total ion chromatogram and the extracted anion profiles of ascorbate, nitrate, and perchlorate. The corresponding mass spectra of each anion are presented in Figures 5.8b-e. The accurate mass data for this sample and the retention time for each analyte are presented in Table 5.8. As figure 5.8a shows, ascorbate elutes at 
3.82 minutes, nitrate at 13.09 minutes, and perchlorate at 22.75 minutes. The chromatographic peak for ascorbate (see Figure 5.8b) contains all three characteristic ions: the molecular anion at $\mathrm{m} / \mathrm{z} 175.0242$, and the fragment anions at $\mathrm{m} / \mathrm{z} 115.0027$ and $\mathrm{m} / \mathrm{z}$ 87.0082. Nitrate has its molecular anion at $\mathrm{m} / \mathrm{z} 61.9877$ and the cluster anion at $\mathrm{m} / \mathrm{z}$ 209.9406 as shown in Figure 5.8c. The ratio of $\mathrm{m} / \mathrm{z} 210$ to $\mathrm{m} / \mathrm{z} 62$ is lower for this sample than for the American Pioneer sample. In Figure 5.7d, perchlorate has a molecular anion at $m / z$ 98.9483, and an isotopic peak at $m / z$ 100.9462. Unlike the American Pioneer sample, chlorate is not present.

Figure 5.9a-b displays data collected for an intact sample of Black Canyon. Figure 5.9a displays the total ion chromatogram and the extracted anion profile of nitrate. The corresponding mass spectrum of nitrate is presented in Figure 5.9b. The accurate mass data for this sample and the retention time for nitrate are presented in Table 5.9. As figure 5.9a shows, nitrate elutes at 12.62 minutes. Nitrate has its molecular anion at $m / z 61.9878$ and the cluster anion at $\mathrm{m} / \mathrm{z} 209.9404$ as shown in Figure 5.9b. The chromatographic peak and corresponding mass spectrum for ascorbate are conspicuously absent from this sample.

Although multiple aliquots from the source of Black Canyon used in this study were tested, not one contained detectable levels of ascorbic acid. Previous work reported that a Black Canyon sample manufactured in 1994 did not contain detectable levels of ascorbic acid, while a sample manufactured in 1996 did (89). The sample of Black Canyon analyzed during the course of the present study was from the same source as the 1994 sample in the Goodpaster study, courtesy of the ATF. At the time, the 1994 sample of Black Canyon was the only available source of the propellant. The lack of detectable 
levels of ascorbic acid in the 1994 sample of Black Canyon was confirmed with two additional techniques, Attenuated Total Reflectance-Fourier Transform Infrared Spectroscopy (ATR-FTIR) and X-Ray Powder Diffraction (XRD). It is therefore likely that although the Black Canyon sample initially contained an ascorbic acid fuel source, the compound degraded over time until the levels of intact ascorbic acid which remained were at levels too low to be detected.

Figure 5.9. Analysis of intact grains of Black Canyon by HPLC-ESI-QToFMS (a) total ion chromatogram and extracted ion chromatogram for nitrate (b) mass spectrum of nitrate.
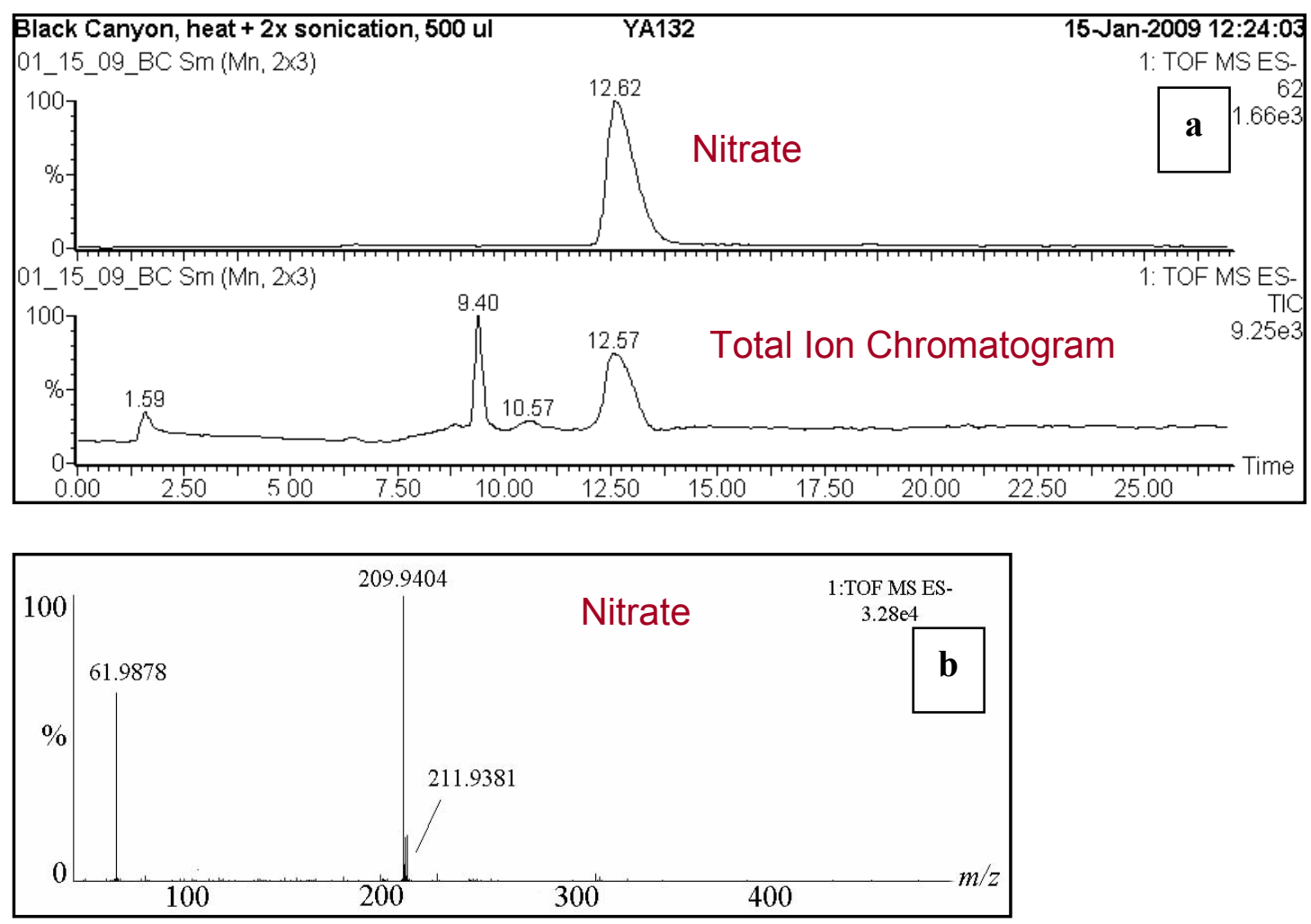
Table 5.9. Accurate mass data for the anions present in intact grains of Black Canyon.

\begin{tabular}{|c|c|c|c|c|}
\hline Component & Calculated $\mathrm{m} / \mathrm{z}$ & Measured $\mathrm{m} / \mathrm{z}$ & $\pm m \mathrm{ma}$ & $\pm \mathrm{ppm}$ \\
\hline Nitrate & 61.9878 & 61.9878 & 0 & 0 \\
\hline
\end{tabular}

Table 5.10. Results of analysis of intact propellant samples by HPLC-ESI-QToFMS.

\begin{tabular}{|c|c|c|c|c|c|c|c|}
\hline & \multicolumn{6}{|c|}{$\boldsymbol{m} / \boldsymbol{z}$} \\
\hline & Oxalate & Threonate & Ascorbate & $\mathrm{NO}_{2}$ & $\mathrm{NO}_{3}$ & $\mathrm{ClO}_{3}$ & $\mathrm{ClO}_{4}$ \\
\hline Propellant & $\mathbf{8 9}$ & $\mathbf{1 3 5}$ & $\mathbf{1 7 5}$ & $\mathbf{4 6}$ & $\mathbf{6 2}$ & $\mathbf{8 3}$ & $\mathbf{9 9}$ \\
\hline American Pioneer & No & No & Yes & No & Yes & Yes & Yes \\
\hline Black Canyon & No & No & No & No & Yes & No & No \\
\hline Black Mag3 & No & No & Yes & No & Yes & No & Yes \\
\hline Clean Shot & No & No & Yes & No & Yes & No & Yes \\
\hline Goex Pinnacle & No & No & Yes & No & Yes & No & Yes \\
\hline Jim Shockey's Gold & No & No & Yes & No & Yes & Yes & Yes \\
\hline
\end{tabular}

The data collected from the analysis of all of the intact propellant samples are summarized in Table 5.10. Of the samples in intact propellant which were analyzed, the ascorbic acid fuel source was detected as the ascorbate anion in five of the six propellants tested. The nitrate oxidizer was detected in all six propellants, while the perchlorate oxidizer was detected in five of the six propellants. Chlorate was also detected in two propellants which contain a perchlorate oxidizer: Jim Shockey’s Gold and American Pioneer. The presence of chlorate in these two intact samples may suggest some degree of powder decomposition had occurred resulting in some of the original perchlorate 
oxidizer being converted to chlorate. These data are in keeping with the patent information available for these products (16) (126) (127) (128) (129), which indicates that while all six propellants contain an ascorbic acid fuel and nitrate oxidizer, only Black Canyon lacks an additional perchlorate oxidizer.

Post-burn residues from all six brands of ascorbic acid-based propellants, including American Pioneer, Black Mag3, Black Canyon, Clean Shot, Goex Pinnacle Replica, and Jim Shockey's Gold were analyzed by HPLC-ESI-QToFMS,. The results of the analysis of post-burn residues from Black Mag3 are presented in Figures 5.10a-f, and the accurate mass data for this sample is present in Table 5.11.

For this sample of Black Mag3, the original ascorbic acid fuel was detected as ascorbate at 3.75 minutes. This chromatographic peak contained two characteristic anions at $m / z$ 175.0236, and $m / z 115.0032$ (see Figure 5.10c). The chromatographic peaks for the oxidizers nitrate and perchlorate were detected at 13.06 minutes and 22.92 minutes, respectively, as shown in Figure 5.10a. The mass spectra for these anions are shown in Figures 5.10f and 5.10g. Nitrate has a molecular anion peak at $\mathrm{m} / \mathrm{z} 61.9875$, and a cluster peak at $\mathrm{m} / \mathrm{z}$ 209.9399. Perchlorate has a molecular anion peak at $\mathrm{m} / \mathrm{z} 98.9487$, and an isotopic peak at $m / z$ 100.9464. Chromatographic peaks for the combustion products nitrite and chlorate were detected at 9.15 minutes and 12.05 minutes, respectively. As shown in Figure 5.10d, nitrite has a molecular anion peak at $\mathrm{m} / \mathrm{z}$ 45.9927. The molecular anion for chlorate occurs at $\mathrm{m} / \mathrm{z} 82.9536$, while an isotopic peak is at $\mathrm{m} / \mathrm{z} 84.9511$ (see Figure 5.10e). 
Figure 5.10. Analysis of post-burn residues from Black Mag3 by HPLC-ESI-QToFMS (a) total ion chromatogram and extracted ion chromatograms for oxalate, threonate, ascorbate, nitrite, chlorate, nitrate, and perchlorate (b) mass spectrum of oxalate and threonate (c) mass spectrum of ascorbate (d) mass spectrum of nitrite (e) mass spectrum of chlorate (f) mass spectrum of nitrate ( $\mathrm{g}$ ) mass spectrum of perchlorate.
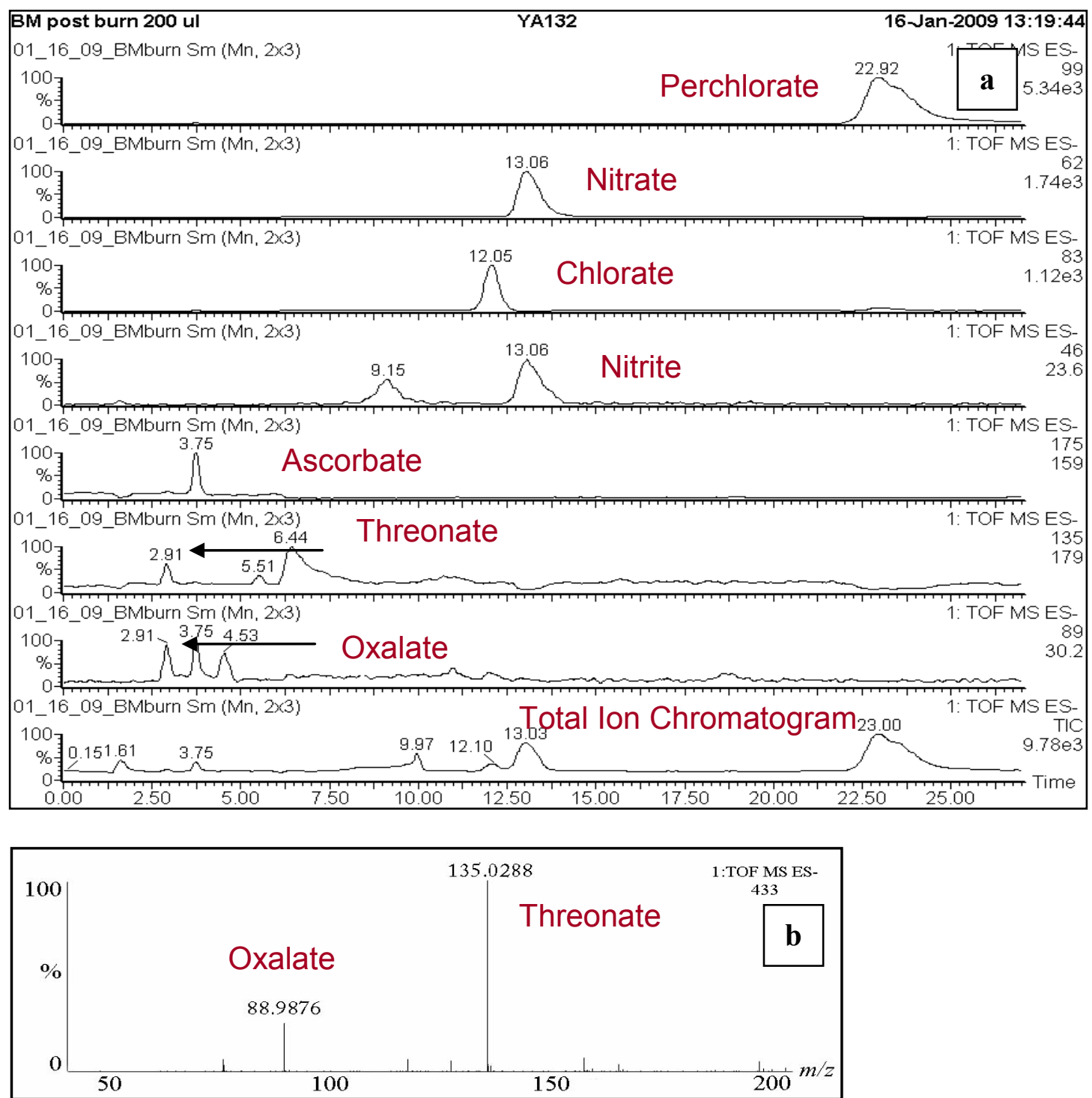

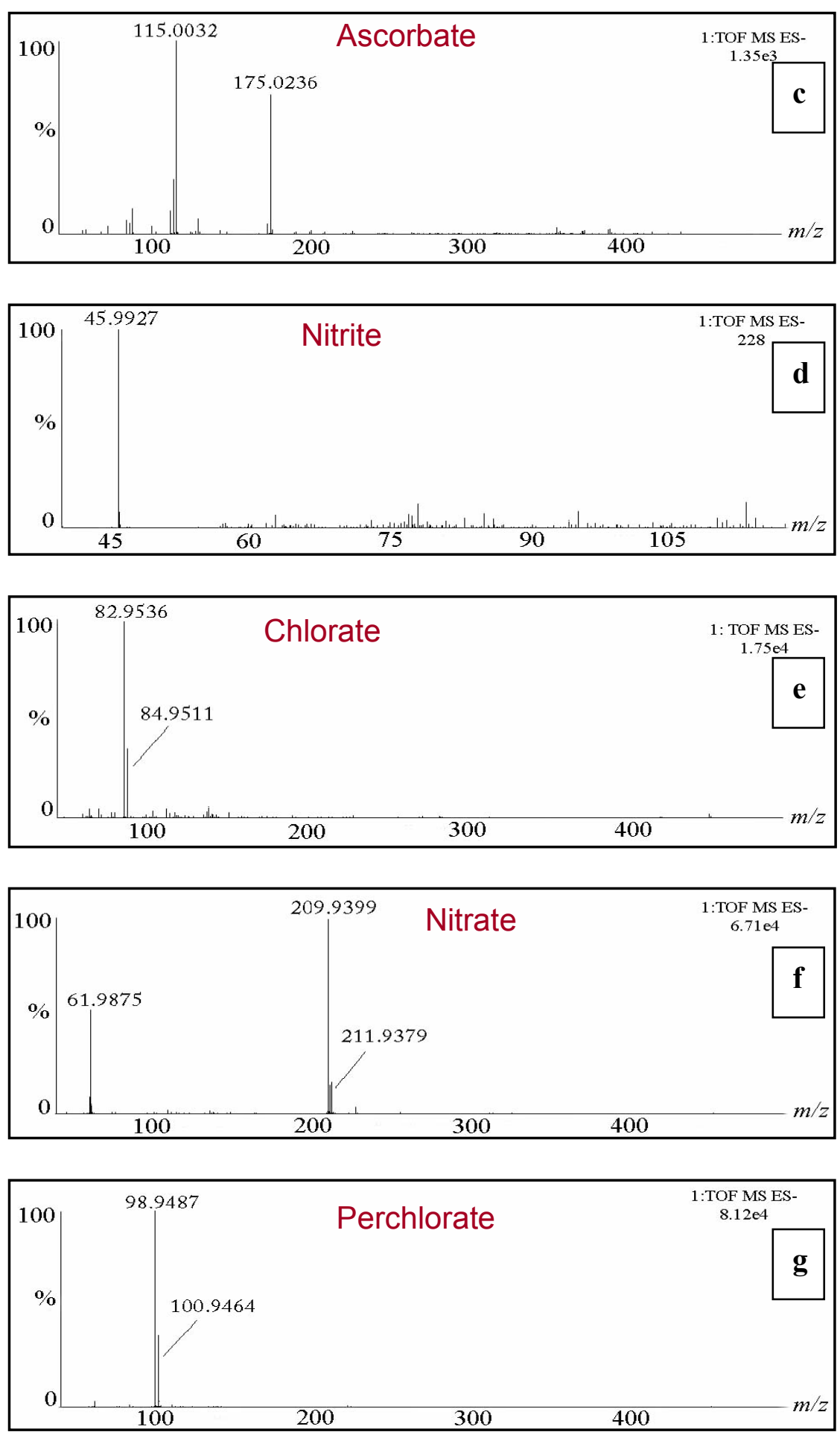
Three separate peaks at 2.91 minutes, 3.75 minutes, and 4.53 minutes were observed in the extracted ion chromatogram for $m / z 89$ as shown in Figure 5.10a. The mass spectrum of the peak at 2.91 minutes is displayed in Figure 5.10b. This peak contains the anionic forms of the degradation products of ascorbic acid, oxalate at $\mathrm{m} / \mathrm{z}$ 88.9876 and threonate at $\mathrm{m} / z$ 135.0288. These results were confirmed when standard samples of oxalate and threonate were analyzed. These anions co-eluted at 2.91 minutes. The peaks at 3.75 minutes and 4.53 minutes could not be identified because the intensities of their mass spectra were too low to obtain accurate mass data. In the extracted ion chromatogram for $m / z 135$, two additional peaks are observed at 5.51 minutes and 6.44 minutes. These peaks also could not be identified because the intensities of their mass spectra were too low to obtain accurate mass data.

Table 5.11. Accurate mass data for the anions present in post-burn residues of Black Mag3.

\begin{tabular}{|c|c|c|c|c|c|}
\hline Component & Rt (min) & Calculated $\mathrm{m} / z$ & Measured $\mathrm{m} / z$ & $\pm m D a$ & $\pm p p m$ \\
\hline Ascorbate & 3.75 & 175.0243 & 175.0236 & -0.3 & -1.7 \\
\hline Oxalate & 2.91 & 88.9875 & 88.9876 & 0.1 & 1.1 \\
\hline Threonate & 2.91 & 135.0294 & 135.0288 & -0.6 & -4.4 \\
\hline Nitrite & 9.15 & 45.9929 & 45.9927 & -0.2 & -4.3 \\
\hline Chlorate & 10.05 & 82.9536 & 82.9536 & 0.0 & 0.0 \\
\hline Nitrate & 13.06 & 61.9878 & 61.9875 & -0.3 & -4.8 \\
\hline Perchlorate & 22.92 & 98.9485 & 98.9487 & 0.2 & 2.0 \\
\hline
\end{tabular}


The results of the analysis of post-burn residues from Clean Shot are presented in Figures 5.11a-f, and the accurate mass data for this sample are presented in Table 5.12. Although intact samples of Clean Shot contained detectable levels of ascorbic acid, analysis of post-burn residues for this propellant did not. The chromatographic peaks for the oxidizers nitrate and perchlorate were detected at 12.92 minutes and 22.46 minutes, respectively, as shown in Figure 5.11a. The mass spectra for these anions are shown in Figures 5.11e and 5.10f. Nitrate has a molecular anion peak at $\mathrm{m} / \mathrm{z} 61.9879$, and a cluster peak at $\mathrm{m} / \mathrm{z}$ 209.9397. Perchlorate has a molecular anion peak at $\mathrm{m} / \mathrm{z} 98.9486$, and an isotopic peak at $m / z$ 100.9465. Chromatographic peaks for the combustion products nitrite and chlorate were detected at 8.76 minutes and 11.96 minutes, respectively. As shown in Figure 5.11c, nitrite has a molecular anion peak at $m / z$ 45.9928. The molecular anion for chlorate occurs at $\mathrm{m} / \mathrm{z} 82.9533$, while an isotopic peak is at $\mathrm{m} / \mathrm{z} 84.9508$ (see Figure 5.11d).

Unlike for the Black Mag3 sample, only a single peak at 2.91 minutes was observed in the extracted ion chromatogram for $m / z 89$ as shown in Figure 5.11a. The mass spectrum of the peak at 2.91 minutes is displayed in Figure 5.11b. This peak contains the anionic forms of the degradation products of ascorbic acid, oxalate at $\mathrm{m} / \mathrm{z}$ 88.9877 and threonate at $m / z$ 135.0294. In the extracted ion chromatogram for $m / z 135$, two additional peaks are observed at 5.36 minutes and 6.42 minutes. These peaks could not be identified because the intensities of their mass spectra were too low to obtain accurate mass data. 
Figure 5.11. Analysis of post-burn residues from Clean Shot by HPLC-ESI-QToFMS (a) total ion chromatogram and extracted ion chromatograms for oxalate, threonate, nitrite, chlorate, nitrate, and perchlorate (b) mass spectrum of oxalate and threonate (c) mass spectrum of nitrite (d) mass spectrum of chlorate (e) mass spectrum of nitrate (f) mass spectrum of perchlorate.
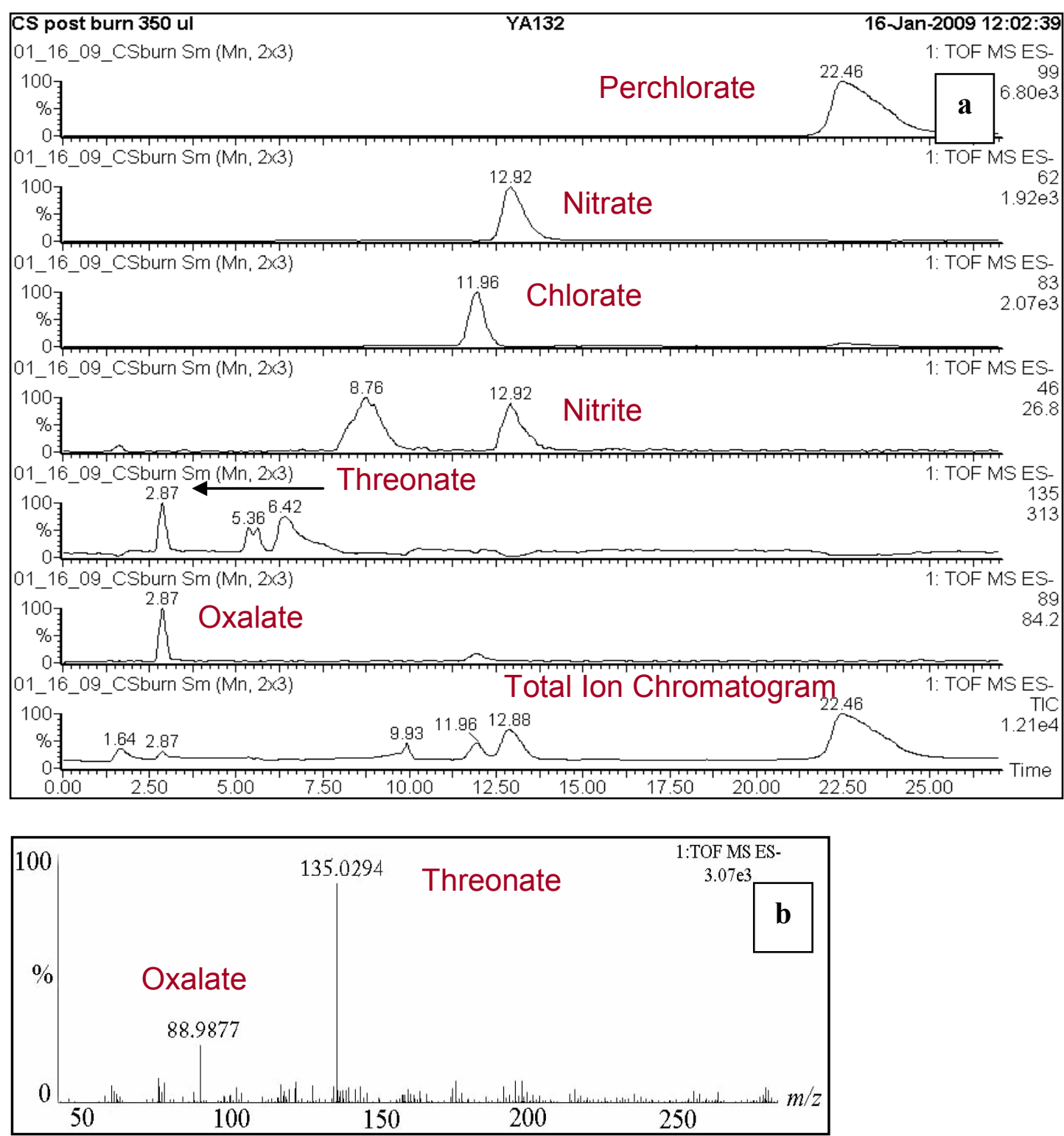

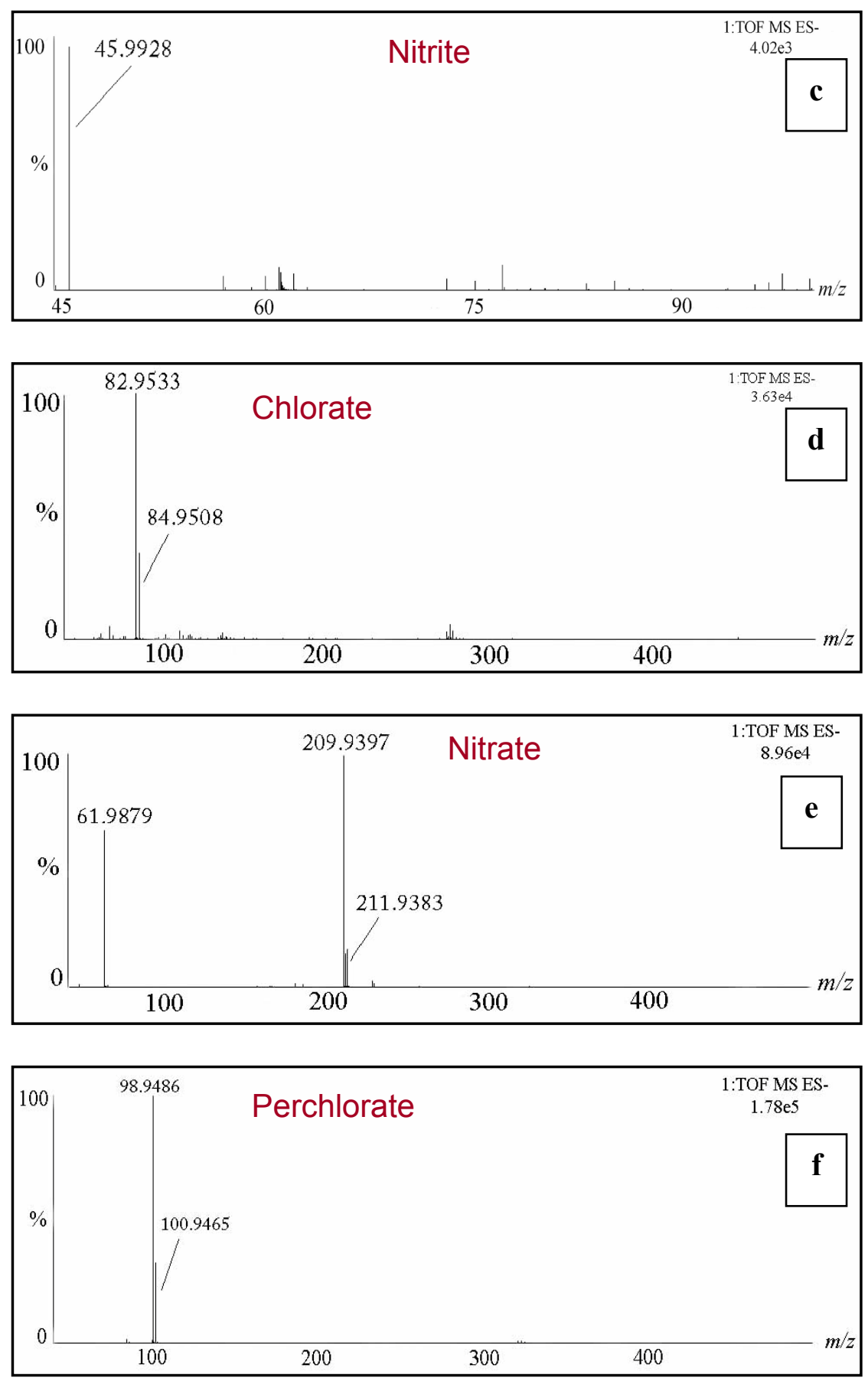
Table 5.12. Accurate mass data for the anions present in post-burn residues of Clean

Shot.

\begin{tabular}{|c|c|c|c|c|c|}
\hline Component & Rt (min) & Calculated $\mathrm{m} / z$ & Measured $\mathrm{m} / z$ & $\pm m \mathrm{mD}$ & $\pm p m$ \\
\hline Oxalate & 2.87 & 88.9875 & 88.9877 & 0.2 & 2.2 \\
\hline Threonate & 2.87 & 135.0294 & 135.0294 & 0.0 & 0.0 \\
\hline Nitrite & 8.76 & 45.9929 & 45.9928 & -0.1 & -2.2 \\
\hline Chlorate & 11.96 & 82.9536 & 82.9533 & -0.3 & -3.6 \\
\hline Nitrate & 12.92 & 61.9878 & 61.9879 & 0.1 & 1.6 \\
\hline Perchlorate & 22.46 & 98.9485 & 98.9486 & 0.1 & 1.0 \\
\hline
\end{tabular}

The data collected from the analysis of all of the post-burn propellant samples are summarized in Table 5.13. Of the six brands of post-burn propellants analyzed, only American Pioneer, Black Mag3, and Goex Pinnacle Replica contained sufficient ascorbic acid to be detected as ascorbate. The lack of detectable levels of ascorbic acid in the samples of Clean Shot and Jim Shockey's Gold was surprising, considering that previous work has shown that ascorbate can be detected in the post-burn residues of these propellants (117). The reason for the discrepancy may be the amount of intact ascorbic acid which remained after burning was insufficient for detection by QToFMS. The degradation products threonic acid and oxalic acid were detected in all five of the ascorbic acid-based propellants, indicating that the ascorbic acid was lost during the deflagration process. There was no ascorbic acid, or degradation products of ascorbic acid, detected in Black Canyon. Thus, the amount of remaining ascorbic acid was below the limit of detection. Nitrate and nitrite were detected in the post-burn residues of all six 
brands of propellant. Chlorate and perchlorate were detected in all of the post-burn samples which contained a perchlorate oxidizer (all propellants except for Black Canyon).

Table 5.13. Results of analysis of post-burn residues by HPLC-ESI-QToFMS. Ascorbate was detected in post-burn residues from three of the six ascorbic acid-based propellants.

\begin{tabular}{|c|c|c|c|c|c|c|c|}
\hline & \multicolumn{6}{|c|}{$\boldsymbol{m} / \mathbf{z}$} \\
\hline & Oxalate & Threonate & Ascorbate & $\mathrm{NO}_{2}$ & $\mathrm{NO}_{3}$ & $\mathrm{ClO}_{3}$ & $\mathrm{ClO}_{4}$ \\
\hline Propellant & $\mathbf{8 9}$ & $\mathbf{1 3 5}$ & $\mathbf{1 7 5}$ & $\mathbf{4 6}$ & $\mathbf{6 2}$ & $\mathbf{8 3}$ & $\mathbf{9 9}$ \\
\hline American Pioneer & Yes & Yes & Yes & Yes & Yes & Yes & Yes \\
\hline Black Mag3 & Yes & Yes & Yes & Yes & Yes & Yes & Yes \\
\hline Black Canyon & No & No & No & Yes & Yes & No & No \\
\hline Clean Shot & Yes & Yes & No & Yes & Yes & Yes & Yes \\
\hline Goex Pinnacle & Yes & Yes & Yes & Yes & Yes & Yes & Yes \\
\hline Jim Shockey's Gold & Yes & Yes & No & Yes & Yes & Yes & Yes \\
\hline
\end{tabular}

Post-blast debris from five of the six PVC pipe bombs, filled with either Jim Shockey's Gold or Goex Pinnacle Replica powders, was analyzed. One pipe bomb filled with Goex Pinnacle Replica powder generated minimal damage when only the end caps fragmented, and was thus excluded from this study. The results of a water extract from Shot III, a pipe which had been filled with Jim Shockey's Gold, are presented in Figures 5.12a-f. The results are similar to what were observed for the post-burn residues. 
Figure 5.12. Analysis of PVC fragments from Shot III by HPLC-ESI-QToFMS

(a) extracted ion chromatograms for oxalate, threonate, nitrite, chlorate, nitrate, and perchlorate (b) mass spectrum of oxalate and threonate (c) mass spectrum of nitrite (d) mass spectrum of chlorate (e) mass spectrum of nitrate (f) mass spectrum of perchlorate.
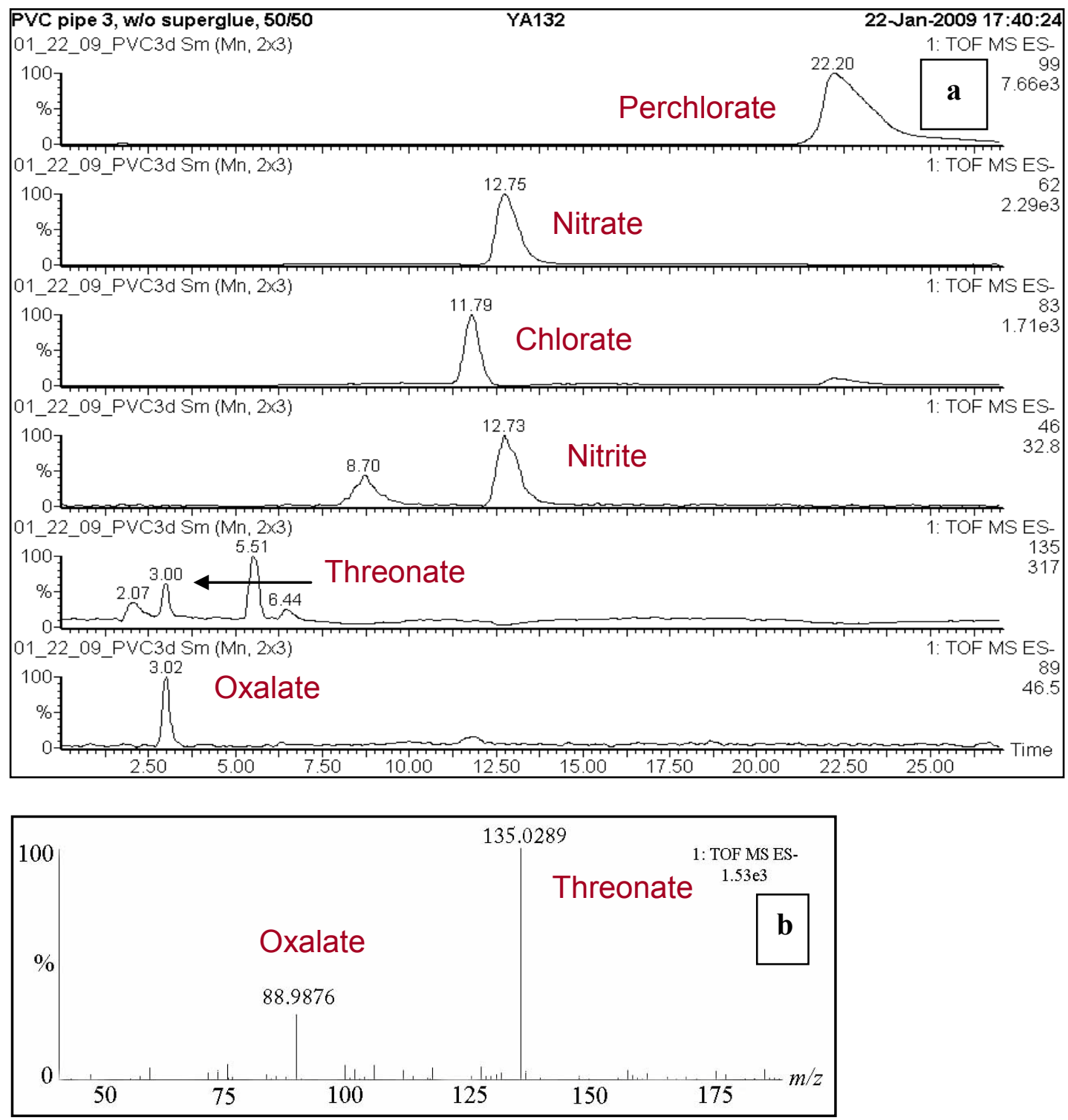

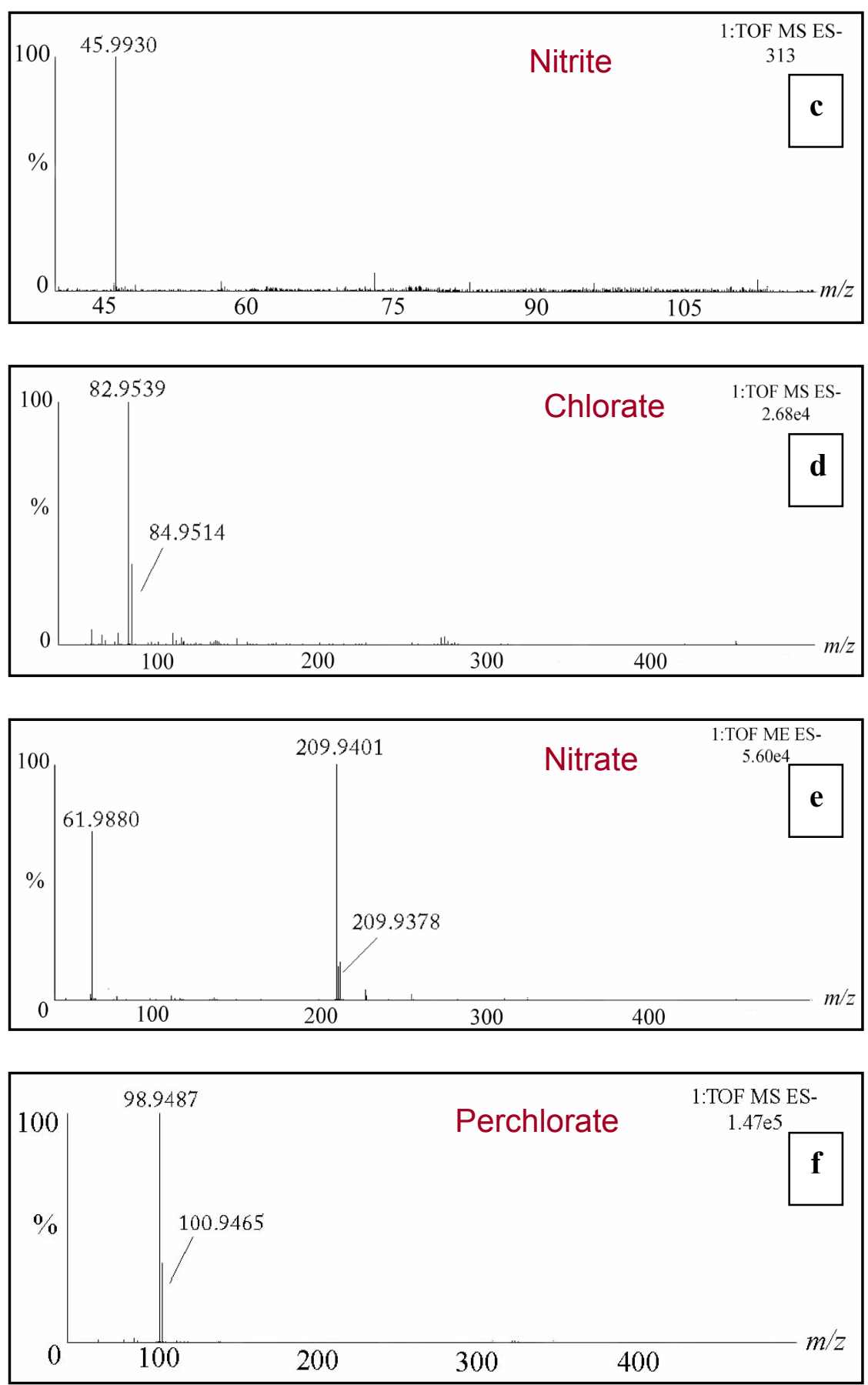
Figure 5.12a shows that the ascorbic acid fuel source was detected via the presence of two of its degradation products, oxalic acid and threonic acid. Only a single peak at 3.02 minutes was observed in the extracted ion chromatogram for $m / z 89$ as shown in Figure 5.12a. The mass spectrum of the peak at 3.02 minutes is displayed in Figure 5.12b. This peak contains the anionic forms of the degradation products of ascorbic acid, oxalate at $\mathrm{m} / \mathrm{z} 88.9876$ and threonate at $\mathrm{m} / \mathrm{z} 135.0289$. In the extracted ion chromatogram for $m / z 135$, three additional peaks are observed at 2.07 minutes, 5.51 minutes and 6.44 minutes. These peaks could not be identified because the intensities of their mass spectra were too low to obtain accurate mass data.

The chromatographic peaks for the oxidizers nitrate and perchlorate were detected at 12.75 minutes and 22.20 minutes, respectively, as shown in Figure 5.12a. The mass spectra for these anions are shown in Figures 5.12e and 5.12f. Nitrate has a molecular anion peak at $\mathrm{m} / \mathrm{z}$ 61.9880, and a cluster peak at $\mathrm{m} / \mathrm{z}$ 209.9401. Perchlorate has a molecular anion peak at $m / z$ 98.9487, and an isotopic peak at $m / z 100.9465$.

Chromatographic peaks for the combustion products nitrite and chlorate were detected at 8.70 minutes and 11.79 minutes, respectively. As shown in Figure 5.12c, nitrite has a molecular anion peak at $\mathrm{m} / \mathrm{z}$ 45.9930. The molecular anion for chlorate occurs at $\mathrm{m} / \mathrm{z}$ 82.9539, while an isotopic peak is at $m / z 84.9514$ (see Figure 5.12d). All measured masses of the anions detected from this post-blast sample are less than $\pm 5 \mathrm{ppm}$ of the calculated masses, as summarized in Table 5.14. 
Table 5.14. Accurate mass data for the anions present in post-blast residues from Shot III. Fragments of PVC pipe were analyzed.

\begin{tabular}{|c|c|c|c|c|}
\hline Component & Calculated $\mathrm{m} / \mathrm{z}$ & Measured $\mathrm{m} / \mathrm{z}$ & $\pm \mathrm{mDa}$ & $\pm \mathrm{ppm}$ \\
\hline Oxalate & 88.9875 & 88.9876 & 0.1 & 1.1 \\
\hline Threonate & 135.0294 & 135.0289 & -0.5 & -3.7 \\
\hline Nitrite & 45.9929 & 45.9930 & 0.1 & 2.2 \\
\hline Chlorate & 82.9536 & 82.9539 & 0.3 & 3.6 \\
\hline Nitrate & 61.9878 & 61.9880 & 0.2 & 3.2 \\
\hline Perchlorate & 98.9485 & 98.9487 & 0.2 & 2.0 \\
\hline
\end{tabular}

The results of a water extract from Shot V, a pipe which had been filled with Goex Pinnacle Replica powder, are presented in Figures 5.13a-f. The results are similar to what were observed for the post-burn residues. Figure 5.13a shows that the ascorbic acid fuel source was detected via the presence of two of its degradation products, oxalic acid and threonic acid. Only a single peak at 3.11 minutes was observed in the extracted ion chromatogram for $\mathrm{m} / \mathrm{z} 89$ as shown in Figure 5.13a. The mass spectrum of the peak at 3.11 minutes is displayed in Figure 5.13b. This peak contains the anionic forms of the degradation products of ascorbic acid, oxalate at $\mathrm{m} / \mathrm{z} 88.9873$ and threonate at $\mathrm{m} / \mathrm{z}$ 135.0297. In the extracted ion chromatogram for $m / z 135$, two additional peaks are observed at 5.69 minutes and 6.45 minutes. These peaks could not be identified because the intensities of their mass spectra were too low to obtain accurate mass data. Two additional peaks were detected for $\mathrm{m} / \mathrm{z} 135$ at 5.69 minutes and 6.45 minutes. These peaks could not be identified because of weak intensities in their respective mass spectra. 
Figure 5.13. Analysis of PVC fragments from Shot V by HPLC-ESI-QToFMS (a) total ion chromatogram and extracted ion chromatograms for oxalate, threonate, nitrite, chlorate, nitrate, and perchlorate (b) mass spectrum of oxalate and threonate (c) mass spectrum of nitrite (d) mass spectrum of chlorate (e) mass spectrum of nitrate (f) mass spectrum of perchlorate.

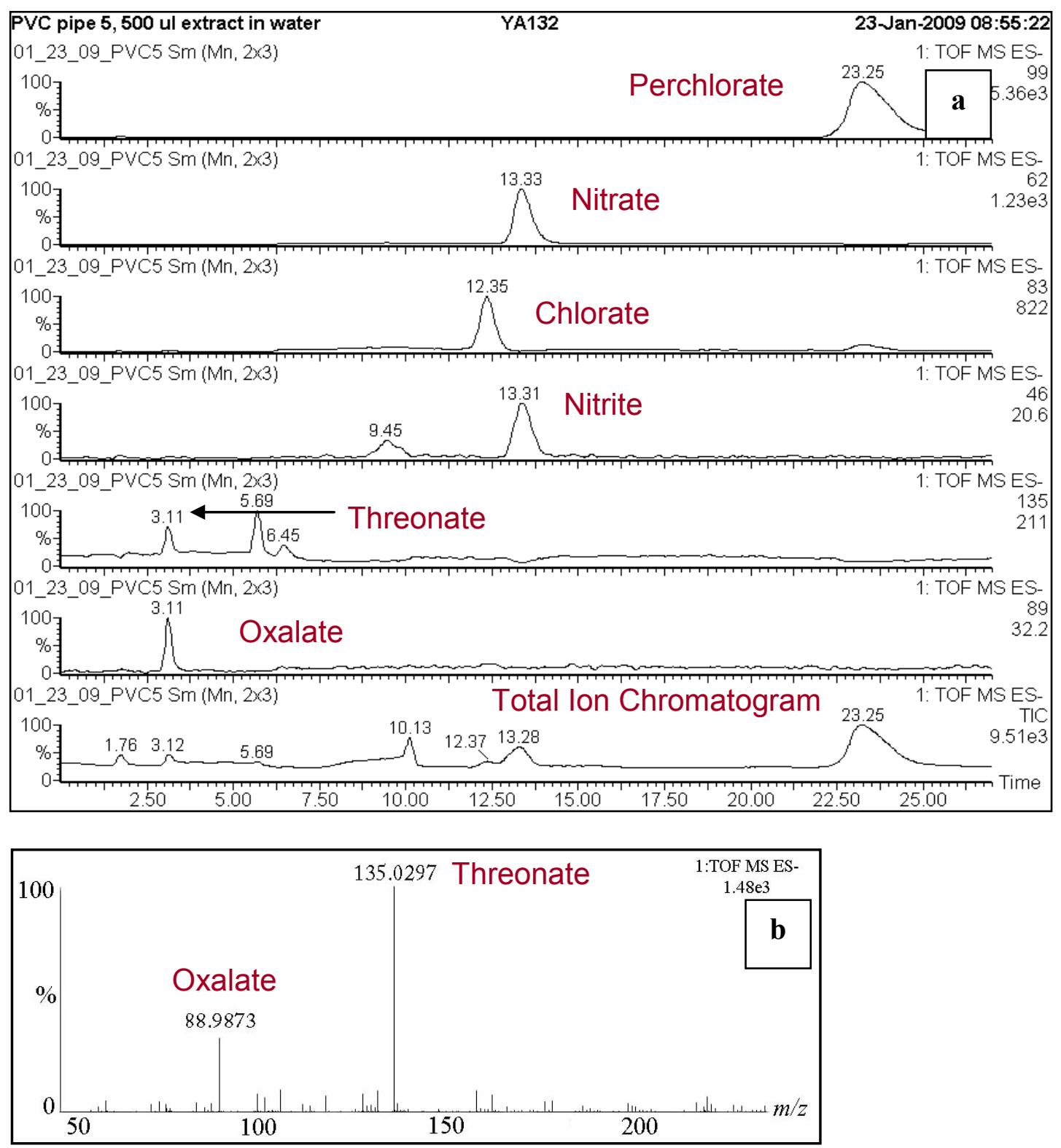



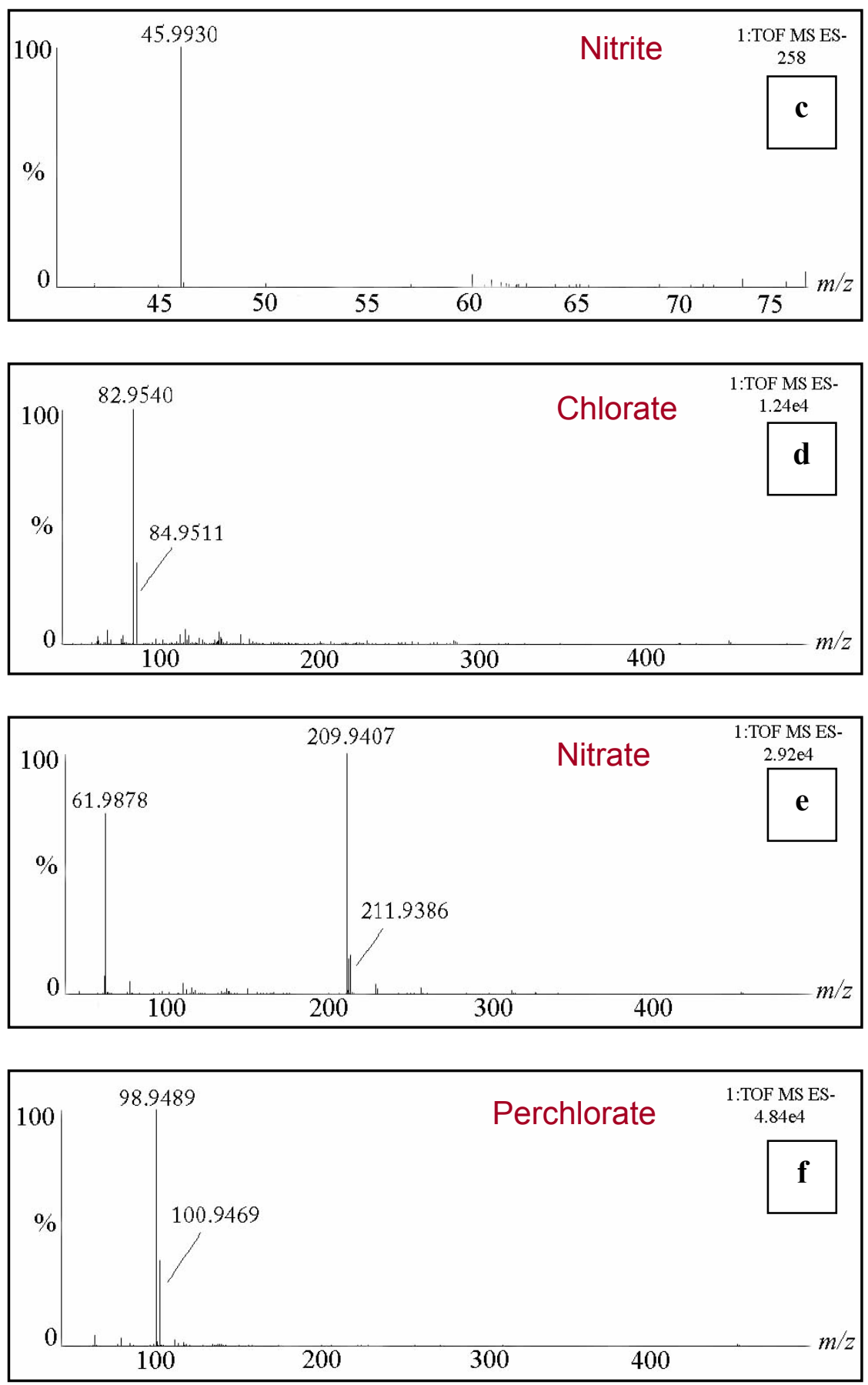
The chromatographic peaks for the oxidizers nitrate and perchlorate were detected at 13.33 minutes and 23.25 minutes, respectively, as shown in Figure 5.13a. The mass spectra for these anions are shown in Figures 5.13e and 5.1f. Nitrate has a molecular anion peak at $\mathrm{m} / \mathrm{z} 61.9878$, and a cluster peak at $\mathrm{m} / \mathrm{z}$ 209.9407. Perchlorate has a molecular anion peak at $m / z$ 98.9489, and an isotopic peak at $m / z 100.9469$.

Chromatographic peaks for the combustion products nitrite and chlorate were detected at 9.46 minutes and 12.35 minutes, respectively. As shown in Figure 5.13c, nitrite has a molecular anion peak at $\mathrm{m} / \mathrm{z} 45.9930$. The molecular anion for chlorate occurs at $\mathrm{m} / \mathrm{z}$ 82.9540, while an isotopic peak is at $m / z 84.9511$ (see Figure 5.13d). All measured masses of the anions detected from this post-blast sample are less than $\pm 5 \mathrm{ppm}$ of the calculated masses as summarized in Table 5.15.

Table 5.15. Accurate mass data for the anions present in post-blast residues from Shot V.

\begin{tabular}{|c|c|c|c|c|c|}
\hline Component & Rt (min) & Calculated $\mathrm{m} / \mathrm{z}$ & Measured $\mathrm{m} / \mathrm{z}$ & $\pm \mathrm{mDa}$ & $\pm \mathrm{ppm}$ \\
\hline Oxalate & 3.11 & 88.9875 & 88.9873 & -0.2 & -2.2 \\
\hline Threonate & 3.11 & 135.0294 & 135.0297 & 0.3 & 2.2 \\
\hline Nitrite & 9.45 & 45.9929 & 45.9930 & 0.1 & 2.2 \\
\hline Chlorate & 12.35 & 82.9536 & 82.9540 & 0.4 & 4.8 \\
\hline Nitrate & 13.33 & 61.9878 & 61.9878 & 0.0 & 0.0 \\
\hline Perchlorate & 23.25 & 98.9485 & 98.9489 & 0.4 & 4.0 \\
\hline
\end{tabular}


The results of post-blast debris analysis for all five PVC bombs are summarized in Table 5.16. The inorganic oxidizers, nitrate and perchlorate, and the inorganic combustion products, nitrite and chlorate, were detected in the post-blast residues of all five sets of debris. The organic ascorbic acid fuel was not detected as the molecular anion ascorbate in the residues of any of the five pipe bombs. However, the degradation products oxalic acid and threonic acid were detected in the residues of all five pipe bombs. The ascorbic acid likely degraded as a result of the heat and pressure of the deflagration event to form oxalic acid and threonic acid. The post-blast PVC fragments had been packaged in metal paint cans and stored at room temperature for approximately fifteen months prior to analysis. This long storage time may also have contributed to the degradation of ascorbic acid. Previous studies found that ascorbic acid could be detected in post-blast debris by IC-IPD up to one year after the initial deflagration even when the fragments were stored at $-15^{\circ} \mathrm{C}(117)$. However, the limit of detection for this method was an order of magnitude lower than the HPLC-ESI-QToFMS method. Refrigeration of the PVC pipe shot debris from the ATF could have helped to preserve ascorbic acid residues. Nonetheless, the presence of oxalic and threonic acid, in addition to the organic oxidizers, indicates these powders originated from an ascorbic-acid based propellant. A brief discussion of the effects of sonication on the detection of ascorbic acid must be made. Sonication of the intact propellants were necessary to dissociate the propellant granules, while sonication of the in post-blast samples was necessary to free the post-blast residues from the dirt and debris of the post-blast fragments. Ascorbic acid degradation peaks at $\mathrm{m} / \mathrm{z} 115$ and $\mathrm{m} / \mathrm{z} 87$ were present in both the sonicated samples and non-sonicated standards. The ascorbic acid degradation compounds oxalate and threonate 
were only present in the deflagrated samples (they were absent from both the standard and the intact propellant samples). The presence of oxalate and threonate did not appear to be affected by sonication, as the compounds were noted in the post-blast samples, which were sonicated, and the post-burn samples, which were not.

Table 5.16. Results of analysis of PVC fragments by HPLC-ESI-QToFMS. Ascorbate was not detected on any of the post-blast PVC fragments.

\begin{tabular}{|c|c|c|c|c|c|c|c|}
\hline & \multicolumn{6}{|c|}{$m / z$} \\
\hline & Oxalate & Threonate & Ascorbate & $\mathrm{NO}_{2}{ }^{-}$ & $\mathrm{NO}_{3}{ }^{-}$ & $\mathrm{ClO}_{3}{ }^{-}$ & $\mathrm{ClO}_{4}{ }^{-1}$ \\
\hline Shot & $\mathbf{8 9}$ & $\mathbf{1 3 5}$ & $\mathbf{1 7 5}$ & $\mathbf{4 6}$ & $\mathbf{6 2}$ & $\mathbf{8 3}$ & $\mathbf{9 9}$ \\
\hline I & Yes & Yes & No & Yes & Yes & Yes & Yes \\
\hline III & Yes & Yes & No & Yes & Yes & Yes & Yes \\
\hline IV & Yes & Yes & No & Yes & Yes & Yes & Yes \\
\hline V & Yes & Yes & No & Yes & Yes & Yes & Yes \\
\hline VI & Yes & Yes & No & Yes & Yes & Yes & Yes \\
\hline
\end{tabular}

\section{Conclusions}

This HPLC-ESI-QToFMS method is a fast, direct, and definitive analytical technique for identifying ascorbic acid-based propellants with minimal sample preparation. The combination of retention time, accurate mass measurements, and mass spectral information of this method facilitates an unequivocal identification of both the oxidizers and the fuel source for intact powders, as well as the original ingredients and combustion products for post-blast residues. The limitations of this method include a high 
detection limit for ascorbate and nitrite, and an inability to detect chloride. However, because low explosives tend to leave copious amounts of residues behind following deflagration, the high LOD values for ascorbate and nitrate may not actually hinder detection of these analytes in real-world samples. Future experiments should involve the analysis of fresh post-blast debris, as well as a study of the effects of different storage materials and conditions on the ability to detect ascorbate in post-blast residues. 


\section{Chapter VI. Optimization of HPLC/ED and HPLC/FD Methods for the Analysis of Hydrogen Peroxide}

\section{A. Introduction}

As a result of rising interest in homeland security and defense, improved technology has been developed for the detection of nitrated explosives. This has led to an increased use of improvised explosive materials which do not contain nitro-groups, so as to avoid detection (17) or to circumvent barriers to the acquisition of commercial explosives. One such class of energetic materials which have recently drawn attention is the peroxide-based explosives. These explosives can be either solids or liquids. They can exist as a blend of separate fuel and oxidizer compounds, such as mixtures of concentrated hydrogen peroxide with carbonaceous fuels. Alternatively, hydrogen peroxide may be used as a starting component in the production of explosives which contain the fuel and oxidizer within the same molecule. Triacetone triperoxide (TATP) and hexamethylene triperoxide diamine (HMTD) are examples of the latter type.

In the event that peroxide-based explosives are used in the commission of a crime or terrorist act, forensic scientists seek to identify the original explosive from the residues which are left behind. A considerable amount of research has been published regarding the development of methods for the analysis of TATP and HMTD (130) (131) (132) (133) (134) (135) (136) (137). Because improvised explosive mixtures containing concentrated hydrogen peroxide have emerged only recently, few published methods for the analysis of post-blast residues from these explosives are available. It was the goal of this research to optimize two complementary methods, based on different principles of separation and detection, which could be employed for the separation and detection of 
trace amounts of hydrogen peroxide. As neither method independently provides the level of certainty which is required for use in a court of law, the methods are best employed concurrently.

\section{Properties of hydrogen peroxide}

Hydrogen peroxide is a strong oxidant. Most municipal and industrial applications utilize $35 \%-50 \%$ concentrated solutions of hydrogen peroxide. Solutions of hydrogen peroxide up to $70 \%$ can be purchased from chemical supply houses. Household applications involving 3\% solutions of hydrogen peroxide include cleaning wounds and bleaching clothing, while the bleaching of hair commonly utilizes $15 \%$ solutions of hydrogen peroxide. Industrial applications of hydrogen peroxide include pulp and paper bleaching, organic and inorganic chemical processing, the treatment of metal, catalysis of polymerization reactions, and industrial waste treatment (138). Hydrogen peroxide can form free radicals through homolytic cleavage of either the $\mathrm{O}-\mathrm{O}$ bond or the $\mathrm{O}-\mathrm{H}$ bond, as seen below in reactions 1 and 2:

(1) $\mathrm{HOOH} \rightarrow \mathrm{H} \cdot+\cdot \mathrm{OOH}$

(2) $\mathrm{HOOH} \rightarrow 2 \cdot \mathrm{OH}$

Reaction 1 tends to dominate in photochemically initiated reactions, as well as in uncatalyzed vapor phase reactions. In catalytic reactions, and particularly in solution, the nature of the reactants determines which free radicals will dominate. The redox system $\mathrm{Fe}(\mathrm{II})-\mathrm{Fe}(\mathrm{III})$ is a widely used catalyst. When it is combined with hydrogen peroxide, it is known as Fenton's Reagent. The chemistry of the Fenton reaction is dependent upon the 
formation of free radicals, as seen in reactions 3 and 4 . This reaction is employed commercially in the treatment of wastewater (139).

$$
\begin{aligned}
& \mathrm{Fe}^{2+}+\mathrm{H}_{2} \mathrm{O}_{2} \rightarrow \mathrm{Fe}^{3+}+\mathrm{OH}^{-}+\cdot \mathrm{OH} \\
& \mathrm{Fe}^{3+}+\mathrm{H}_{2} \mathrm{O}_{2} \rightarrow \mathrm{Fe}^{2+}+\cdot \mathrm{OOH}+\mathrm{H}^{+}
\end{aligned}
$$

Anhydrous hydrogen peroxide is a clear, colorless liquid of nearly the same dielectric constant and viscosity of water, though it has a greater density than water. Though stable if uncontaminated, hydrogen peroxide solutions will decompose slowly into oxygen gas and water with the evolution of heat. There is considerable evidence that this process occurs as a chain reaction involving free radicals (139). Decomposition of hydrogen peroxide into perhydroxy and hydroxyl radicals is accelerated in the presence of near UV light. In dilute solutions, the water which is present can absorb the heat which is evolved through decomposition of hydrogen peroxide. In more concentrated solutions, the heat evolved from decomposition raises the temperature of the solution, which in turn increases the rate of decomposition. High alkalinity can also increase the decomposition rate of hydrogen peroxide. The catalytic decomposition of hydrogen peroxide at a concentration of $70 \%$ or greater proceeds rapidly, and with sufficient heat released that the products are oxygen and steam. The thrust from this reaction can be used to propel torpedoes and small missiles (139).

Great care must be taken when handling concentrated hydrogen peroxide. Though hydrogen peroxide itself will not support combustion, the decomposition of hydrogen peroxide liberates oxygen, which will support combustion. A fire can result if concentrated hydrogen peroxide is brought into contact with combustible material such as paper or wood (22) (140). Other materials which are incompatible with hydrogen 
peroxide include reducing agents, organic materials, alkalis, many metals, rust, and dirt. Stabilizers such as acetanilide and phosphoric acid are often added to hydrogen peroxide during the manufacturing process to inhibit the catalytic decomposition effects of metals and other impurities which may contaminate the chemical during transportation, storage, and handling (140).

In acidic solution, the standard reduction potential for hydrogen peroxide is $\mathrm{E}_{0}=+1.80 \mathrm{~V}$ (see reaction 5) (139), while the standard reduction potential for water is $E_{0}=-0.83 \mathrm{~V}$ (see reaction 6).

$$
\begin{aligned}
& \mathrm{H}_{2} \mathrm{O}_{2}+2 \mathrm{H}^{+}+2 e^{-} \rightarrow 2 \mathrm{H}_{2} \mathrm{O} \\
& 2 \mathrm{H}_{2} \mathrm{O}+2 e^{-} \rightarrow \mathrm{H}_{2}(\mathrm{~g})+2 \mathrm{OH}^{-}
\end{aligned}
$$

Because of problems with the reduction of dissolved oxygen in the mobile phase at this potential, as well as the difficulty in finding a working electrode which can handle such strongly positive potentials, detection of hydrogen peroxide is much more effective when performed in oxidative mode (65).

At $\mathrm{pH}=0$, when hydrogen peroxide is in the form $\mathrm{H}_{2} \mathrm{O}_{2}$, the oxidation of hydrogen peroxide will occur at $\mathrm{E}_{0}=-0.66 \mathrm{~V}$ (see reaction 7). Because hydrogen peroxide is a weak acid with a $\mathrm{pK}_{\mathrm{a}}$ of 11.65 , in strongly basic solution, hydrogen peroxide will form the perhydroxyl ion $\mathrm{HOO}^{-}$(see reaction 8a). At $\mathrm{pH}=14$, when hydrogen peroxide is in the form $\mathrm{HOO}^{-}$, the oxidation of hydrogen peroxide will occur at $\mathrm{E}_{0}=+0.08 \mathrm{~V}$ (see reaction $8 \mathrm{~b}$ ) (139). At $\mathrm{pH}=14$, oxidation of hydrogen peroxide to oxygen is accomplished with the concomitant reduction of hydroxide ions to water. This reaction can be combined with reaction 8 a to yield reaction 9 , which represents the nature of the oxidation reaction of hydrogen peroxide at the surface of the working electrode. 


$$
\begin{aligned}
& \mathrm{H}_{2} \mathrm{O}_{2} \rightarrow 2 \mathrm{H}^{+}+\mathrm{O}_{2}+2 e^{-} \\
& \mathrm{H}_{2} \mathrm{O}_{2}+\mathrm{OH}^{-} \rightarrow \mathrm{HOO}^{-}+\mathrm{H}_{2} \mathrm{O} \\
& \mathrm{OH}^{-}+\mathrm{OOH}^{-} \rightarrow \mathrm{O}_{2}+\mathrm{H}_{2} \mathrm{O}+2 e^{-} \\
& \mathrm{H}_{2} \mathrm{O}_{2}+2 \mathrm{OH}^{-} \rightarrow \mathrm{O}_{2}+2 \mathrm{H}_{2} \mathrm{O}+2 e-
\end{aligned}
$$

At high working electrode potentials, the oxidation of water and other mobile phase components can overwhelm the signal generated from the oxidation of hydrogen peroxide. A working potential must be found which is high enough to oxidize hydrogen peroxide, but low enough to avoid the problem of oxidation of the mobile phase.

\section{Previous Research}

The need to detect the presence of improvised peroxide mixtures both pre-blast and post-blast has resulted in the on-going research and development of novel techniques for both trace and bulk analysis. Historically, atmospheric hydrogen peroxide and selected organic peroxides have been analyzed using high performance liquid chromatography with post-column derivatization and fluorescence detection (141) (142) (143) (144). These chemicals are of interest because they have been linked to the formation of acid rain, and have strongly toxic effects on plants (144). One common indirect method of detection of trace levels of hydrogen peroxide is to utilize the reaction of hydrogen peroxide with $p$-hydroxyphenylacetic acid in the presence of an iron containing catalyst (141) (142) (143) (144) (145). The product of this reaction is the dimer 6,6'-dihydroxy-3,3'-biphenyldiacetic acid, the anionic form of which is readily detectable using a standard HPLC fluorescence detector. 
Hellpointner and Gaeb (142) were the first group to utilize a reversed phase HPLC separation followed by a post-column reaction with horseradish peroxidase and $p$ hydroxyphenylacetic acid (POPHA) for the analysis of hydrogen peroxide and atmospheric organic peroxides. The product of this reaction was a dimer of $p$ hydroxyphenylacetic acid; the anionic form of the dimer was fluorescent. A cold sulfuric acid/EDTA mobile phase was employed to stabilize and separate the organic peroxides. The $\mathrm{pH}$ and temperature of the eluent were increased post-column to convert the hydroxyalkyl hydroperoxides into hydrogen peroxide. Following formation of the dimer, sodium hydroxide was added to the flow stream prior to reaching the detector. An excitation wavelength of $286 \mathrm{~nm}$ and an emission wavelength of $404 \mathrm{~nm}$ were employed for the detection of the fluorescent dimer. The authors cited a limit of detection for hydrogen peroxide of $1.4 \mu \mathrm{M}$.

Kok et al. (143) utilized a very similar procedure to Hellpointner and Gaeb (142) for the analysis of hydrogen peroxide and ten organic peroxides. A fluorescence detector with an excitation wavelength of $330 \mathrm{~nm}$ and an emission wavelength of $400 \mathrm{~nm}$ was employed for the detection of the POPHA dimer. Under these conditions, only six of the eleven peroxides could be separated within fifteen minutes of injection. The presence of EDTA in the mobile phase allowed for the complexation of any metal ions which were present, thereby minimizing degradation of the peroxides prior to detection The authors cited a limit of detection of $90 \mathrm{nM}$ for hydrogen peroxide, and $20 \mathrm{nM}$ for the organic peroxides.

Qi et al. (144) utilized a hemin catalyst for the detection of hydrogen peroxide, and methyl hydroperoxide. Hydroxymethyl hydroperoxide and bis(hydroxymethyl) 
peroxide were also detected after conversion to hydrogen peroxide in alkaline solution. The separation involved a phosphoric acid mobile phase, with a C-18 column that was cooled to $1^{\circ} \mathrm{C}$ (presumably to stabilize the organic peroxides). The reagent solution contained hemin and $p$-hydroxyphenylacetic acid in an ammonia buffer. It was determined that the peroxidatic activity of hemin was enhanced by the presence of ammonia (as compared to a glycine-sodium hydroxide buffer). The accessibility of the lone pair of electrons on the nitrogen played an important role in the interaction of the nitrogen base with the heme-iron in the enzyme substrate intermediate adduct (146). The excitation and emission wavelengths of the fluorescence detector were not reported. The authors cited a limit of detection of $9 \mathrm{nM}$ for hydrogen peroxide.

A study by Francois et al. in 2005 (141) utilized a similar procedure to that of Qi (144) for the analysis of hydrogen peroxide and several organic peroxides in air. This approach utilized a phosphoric acid mobile phase and a C-18 analytical column. The column, the mobile phase, the buffer, and the reagent solutions were maintained at $4^{\circ} \mathrm{C}$. The excitation wavelength was listed as $320 \mathrm{~nm}$ and the emission wavelength was listed as $299 \mathrm{~nm}$ (although this is suspected to be incorrect). The authors reported a limit of detection of $7.3 \times 10^{-3} \mu \mathrm{g} / \mathrm{m}^{3}$ for hydrogen peroxide in air.

The use of the hemin/peroxide/ $p$-hydroxyphenylacetic acid reaction was adapted to the analysis of TATP and HMTD by Schulte-Ladbeck et al. (145). Their method involved pre-treating the samples with catalase to degrade any hydrogen peroxide which may have been present to prevent interference in the measurement. The samples were exposed to UV radiation at $254 \mathrm{~nm}$ to decompose the organic peroxides into hydrogen peroxide. The hydrogen peroxide underwent a reaction with $p$-hydroxyphenylacetic acid 
and horseradish peroxidase in solution. An excitation wavelength of $320 \mathrm{~nm}$ and an emission wavelength of $405 \mathrm{~nm}$ were used. This method yielded a limit of detection of $800 \mathrm{nM}$ for TATP and $800 \mathrm{nM}$ for HMTD. An alternative procedure involved the reaction of the UV-irradiated samples with horseradish peroxidase and 2,2'-azino-bis(3ethylbenzothiazoline)-6-sulfonate. The product of this colorimetric reaction was detected with a hand-held photometer. The limit of detection for this method was $8 \mu \mathrm{M}$ for TATP, and $80 \mu \mathrm{M}$ for HMTD. It should be noted that these methods were only used to analyze intact TATP and HMTD samples, and that no post-blast samples were studied. These methods were also applied to the analysis of four different brands of laundry detergent. No false positives were noted.

Further developments were made to the application of the hemin/peroxide/phydroxyphenylacetic acid reaction for the detection of TATP and HMTD in 2003 by Schulte-Ladbeck et al. (58). The revised method included an HPLC separation of TATP and HMTD on a C-18 column with a mobile phase which was $65 \%$ acetonitrile and $35 \%$ water, followed by photochemical degradation of TATP and HMTD to form hydrogen peroxide. The peroxide was subsequently reacted with horseradish peroxidase and $p$ hydroxyphenylacetic acid to form the anionic, fluorescent dimer. This method was successfully applied to the analysis of intact and post-blast HMTD and TATP samples. The limit of detection for both compounds was $2 \mu \mathrm{M}$.

Schulte-Ladbeck et al. (65) published a study in 2003 detailing a method for the detection of TATP and HMTD by reversed phase HPLC with post-column UV irradiation and electrochemical detection. A C-18 analytical column was used in conjunction with a mobile phase which consisted of $65 \%$ acetonitrile and $35 \%$ aqueous $4 \mathrm{mM}$ phosphate 
buffer at $\mathrm{pH}$ 8. Following separation, TATP and HMTD were degraded to hydrogen peroxide by exposure to UV light at $254 \mathrm{~nm}$. Hydrogen peroxide was detected using an applied potential of $+900 \mathrm{mV}$ versus a palladium-hydrogen reference electrode. The authors utilized a glassy carbon working electrode for this application because of its resistance to deactivation, which could have led to a decrease in sensitivity. The authors reported a limit of detection of $3 \mu \mathrm{M}$ for TATP and HMTD. It should be noted that these methods were only used to analyze intact TATP and HMTD, and that no post-blast samples were studied.

While work has been performed on the use of electrochemical detection to analyze hydrogen peroxide in biological samples (147), there has been little peerreviewed work published on the use of this technology for the analysis of hydrogen peroxide in improvised explosives. Many biological-based assays require the use of specially modified electrodes (148), which may be impractical for use in forensic laboratories. Peer-reviewed published methods do exist for the analysis of many of the types of fuel sources which might be utilized in the construction of improvised explosive mixtures, including sugar (70), flour (149), coffee (150), spices (151), ethanol (152), and nitromethane (153). Ideally, both the fuel and oxidizer component of an improvised explosive mixture would be detected during the same chromatographic analysis. However, the sheer variety of fuel components available makes this a daunting task. Currently, there are a very small number of peer-reviewed studies which explore the explosive properties of concentrated hydrogen peroxide/fuel mixtures. Schreck et al. (154) published a study which analyzed peroxide/alcohol mixtures. This study explored the thermal explosive range of mixtures of 2-propanol, 2-methyl-2-propanol, 2-methyl-2- 
butanol, and 2-methyl-2-pentanol with aqueous hydrogen peroxide in various ratios by heating them under confinement. While all of the peroxide/alcohol mixtures resulted in a violent explosion, the combination of hydrogen peroxide and 2-methyl-2-propanol was found to be particularly dangerous over the largest concentration range. It is expected that similar studies involving other types of fuels will be published in the near future.

\section{B. Development of HPLC/ED Method}

\section{Selection of Analytical Column}

Because of specific concerns regarding mixtures of concentrated hydrogen peroxide with sugars, the starting point for development of the HPLC/ED method was the standard method for carbohydrate analysis by the International Commission for Uniform Methods of Sugar Analysis (ICUMSA), as described by Dionex Application Note 92 (71). This method utilized a Dionex CarboPac PA1 guard and analytical column, a 150 $\mathrm{mM} \mathrm{NaOH}$ mobile phase at $\mathrm{pH} 12.4$, and pulsed amperometric detection with a gold working electrode. The Dionex CarboPac PA1 guard column $(4.6$ x $50 \mathrm{~mm})$ and CarboPac PA1 analytical column $(4.6 \times 250 \mathrm{~mm})$ were utilized in the initial experiments for the separation of carbohydrates and hydrogen peroxide. The CarboPac PA1 column packing material was comprised of a polystyrene/divinylbenzene substrate ( $2 \%$ cross linked) with quaternary ammonium functionality. Given that the $\mathrm{pK}_{\mathrm{a}}$ of hydrogen peroxide is 11.65 , the hydrogen peroxide will lose a proton to become ionized when in basic solution, with the extent of peroxide ionization being dependent upon the $\mathrm{pH}$ of the solution. The anionic peroxide then interacts with the quaternary ammonium 
functionality of the column phase packing material to a sufficient degree that baseline separation of the compound from the water peak is achievable.

When the CarboPac PA1 column was used with the $150 \mathrm{mM} \mathrm{NaOH}$ mobile phase, the hydrogen peroxide peak exhibited a sizeable amount of tailing. Because none of the carbohydrates which were tested exhibited this effect, the tailing was postulated to result from the specific chemistry of the interaction of the hydrogen peroxide between the column and mobile phase. Over the course of approximately three months and three hundred injections, the PA1 column began to fail and the tail on the peroxide peak lengthened with each injection. The manufacturer of the column suggested that the column was poisoned by the combination of repeated exposure to the oxidizing properties of both hydrogen peroxide and dissolved oxygen present in the mobile phase.

The CarboPac PA1 guard and analytical columns were replaced with the more robust CarboPac PA10 guard column $(4.6 \times 50 \mathrm{~mm})$ and analytical column $(4.6 \times 250$ $\mathrm{mm})$. The CarboPac PA10 column packing material was comprised of a polystyrene/divinylbenzene substrate (55\% cross linked) with quaternary ammonium functionality; the additional cross-linking makes the column more rigid against high column back-pressure. According to the manufacturer, the additional cross-linking of the column packing material should also make the column more resistant to oxygen poisoning. As the PA10 column contained the same functional groups as the PA1 column, it was speculated that hydrogen peroxide should interact with the two columns in a similar manner. The combination of the PA10 column with the $150 \mathrm{mM} \mathrm{NaOH}$ mobile phase resulted in a successful separation of hydrogen peroxide. 
Two additional columns were also tested for use with the electrochemical detector in PAD mode, including a C-18 column ( 4.6 x $250 \mathrm{~mm})$, and a bare silica column $(2.1 \mathrm{x}$ $150 \mathrm{~mm}$ ). The $\mathrm{C}-18$ column was tested with four different mobile phase compositions: $1.00 \mathrm{mM} \mathrm{H}_{2} \mathrm{SO}_{4}$ with $0.10 \mathrm{mM}$ EDTA; $150 \mathrm{mM} \mathrm{NaOAc} ; 20 \%$ methanol with $80 \%$ water; and $20 \%$ methanol with $80 \%$ water which contained $0.1 \%$ formic acid. Each mobile phase composition was selected because of known advantages. The sulfuric acid/EDTA mobile phase is known to result in a successful separation of hydrogen peroxide when paired with the C-18 column. The $150 \mathrm{mM} \mathrm{NaOAc}$ mobile phase leads to a successful separation when paired with the CarboPac PA10 column. Previous research demonstrated that a methanol/water mobile phase could be used to separate organic peroxides on a C18 column (65). The methanol/water/formic acid mobile phase was selected because this mobile phase has proven successful at resolving organic explosives on a C-18 column (155). The C-18 column/mobile phase experiments were unsuccessful for all four mobile phase compositions. Hydrogen peroxide could not be detected on the C-18 system when it was combined with the $1.00 \mathrm{mM} \mathrm{H}_{2} \mathrm{SO}_{4} / 0.10 \mathrm{mM}$ EDTA mobile phase, presumably because the mobile phase was too dilute to conduct current through the flow cell of the electrochemical detector. Hydrogen peroxide was not well resolved in a reproducible manner when the $\mathrm{C}-18$ mobile phase was used with the other three mobile phase compositions.

In contrast, the silica column did yield some separation of hydrogen peroxide when it was paired with the $150 \mathrm{mM} \mathrm{NaOAc}$ mobile phase. However, detector response for hydrogen peroxide was ten times lower than for the pairing of this mobile phase with the PA10 column. Addition of up to $40 \%$ acetonitrile to the mobile phase failed to change 
the retention time of hydrogen peroxide on the silica column, and only resulted in further suppression of the peroxide signal. This indicated that some of the hydrogen peroxide was adsorbing onto the surface of the silica stationary phase. From these results, it was determined that the CarboPac PA10 column offered a superior separation of hydrogen peroxide in comparison to the silica and C-18 columns. Development of the HPLC-ED method continued with this column.

\section{Development of $150 \mathrm{mM} \mathrm{NaOH} / 150 \mathrm{mM}$ NaOAc mobile phase with electrochemical detection in PAD mode a. Optimization of the mobile phase}

The standard $150 \mathrm{mM} \mathrm{NaOH}$ mobile phase from the Dionex application note on the ICUMSA method for the analysis of sugars in molasses (71) was taken as the starting point for the separation of hydrogen peroxide and glucose due to specific concerns regarding mixtures of concentrated hydrogen peroxide and sugars. The combination of this mobile phase with the CarboPac PA10 column permitted baseline resolution of peroxide from a glucose standard. However, although the glucose peak displayed a Gaussian shape, the peroxide peak displayed non-Gaussian shape, in the form of a significant tail from 3.5-5.5 minutes. The peak symmetry was calculated using the following equation:

peak symmetry $=($ area before top of peak $) \div($ area after top of peak $)$

The combination of the $150 \mathrm{mM} \mathrm{NaOH}$ mobile phase and CarboPac PA10 column resulted in a value for the peak symmetry of hydrogen peroxide which was equal to 0.39 . 
Increasing the flow rate from $1.0 \mathrm{~mL} / \mathrm{min}$ to $1.25 \mathrm{~mL} / \mathrm{min}$ did not have any noticeable effect on reducing the length of the tail on the peroxide peak (see Figure 6.1).

Figure 6.1. Effects of analytical column, mobile phase, and flow rate on the peak shape and retention time of $300 \mathrm{ppm}$ hydrogen peroxide (1) $150 \mathrm{mM} \mathrm{NaOH}, \mathrm{Vm}=1.00$ $\mathrm{mL} / \mathrm{min}$, PA1 Rt $2.853 \mathrm{~min}$ (2) $100 \mathrm{mM} \mathrm{NaOH}, \mathrm{Vm}=1.23 \mathrm{~mL} / \mathrm{min}$, PA1, Rt $3.475 \mathrm{~min}$ (3) $200 \mathrm{mM} \mathrm{NaOH}, \mathrm{Vm}=1.00 \mathrm{~mL} / \mathrm{min}, \mathrm{PA} 1$, Rt $3.076 \mathrm{~min}$ (4) $200 \mathrm{mM} \mathrm{NaOH}, \mathrm{Vm}=$ $1.25 \mathrm{~mL} / \mathrm{min}$, PA1, Rt $2.485 \mathrm{~min}$ (5) $150 \mathrm{mM} \mathrm{NaOH}, \mathrm{Vm}=1.00 \mathrm{~mL} / \mathrm{min}$, PA10, Rt 3.536. The retention time and peak shape of hydrogen peroxide with the PA10 column and $150 \mathrm{mM} \mathrm{NaOH}$ mobile phase at $1.00 \mathrm{~mL} / \mathrm{min}$ are superior to the other column/mobile phase/flow rate combinations.

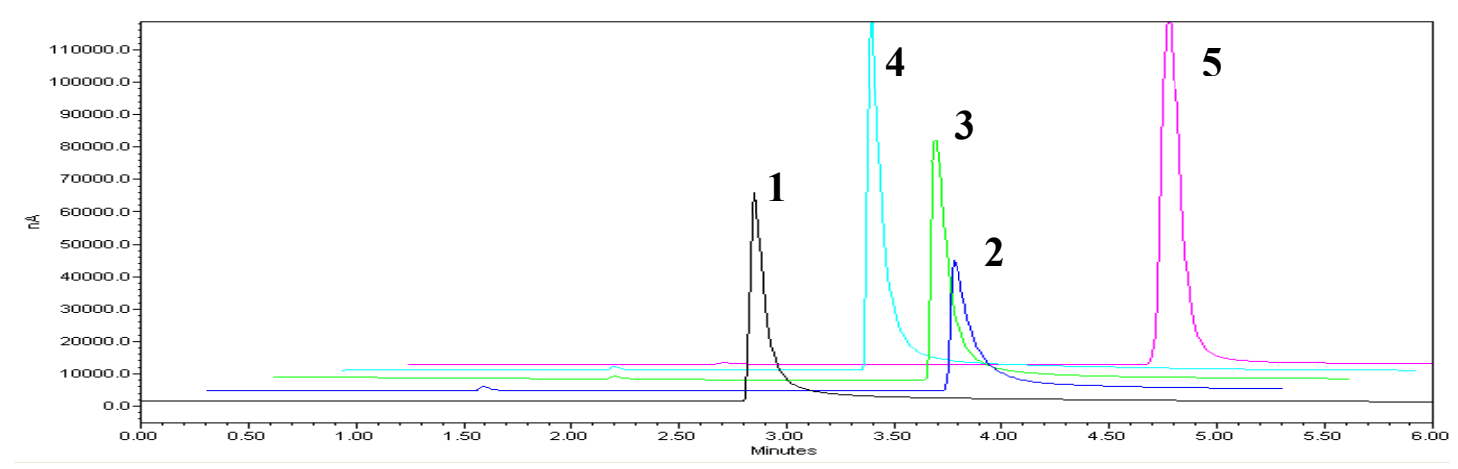

The addition of up to $20 \%$ methanol to the mobile phase did reduce the length of the tail on the peroxide peak, but the peroxide signal was suppressed considerably. Sodium acetate was added to the mobile phase to increase its ionic strength as suggested by a Dionex application update (156). The new mobile phase, which consisted of 100 $\mathrm{mM} \mathrm{NaOH}$ and $100 \mathrm{mM} \mathrm{NaOAc}$, decreased the amount of tailing on the hydrogen peroxide peak without increasing the strength of the peroxide signal. Increasing the 
concentration of the mobile phase to $150 \mathrm{mM} \mathrm{NaOH}$ and $150 \mathrm{mM} \mathrm{NaOAc}$ reduced the amount of peak tailing even further (see Figure 6.2). Use of the $150 \mathrm{mM} \mathrm{NaOH}$ with 150 $\mathrm{mM}$ NaOAc mobile phase at $\mathrm{pH} 12.4$ with the CarboPac PA10 column resulted in a loss of resolution of the carbohydrate standards. Glucose, sucrose, and fructose could no longer be resolved from each other. Instead, they co-eluted at 1.82 minutes. However, baseline separation from hydrogen peroxide was achieved.

Figure 6.2. Effect of mobile phase concentration on peak shape and retention time of 0.5 ppm hydrogen peroxide standard (1) $150 \mathrm{mM} \mathrm{NaOH} / 150 \mathrm{mM} \mathrm{NaOAc}, \mathrm{Vm}=1.00$ $\mathrm{mL} / \mathrm{min}$, PA10, Rt $2.249 \mathrm{~min}$ (2) $100 \mathrm{mM} \mathrm{NaOH} / 100 \mathrm{mM} \mathrm{NaOAc}, \mathrm{Vm}=1.00 \mathrm{~mL} / \mathrm{min}$, PA10, Rt 2.823 min. The peak shape of hydrogen peroxide is superior with $150 \mathrm{mM}$ $\mathrm{NaOH} / 150 \mathrm{mM} \mathrm{NaOAc}$.

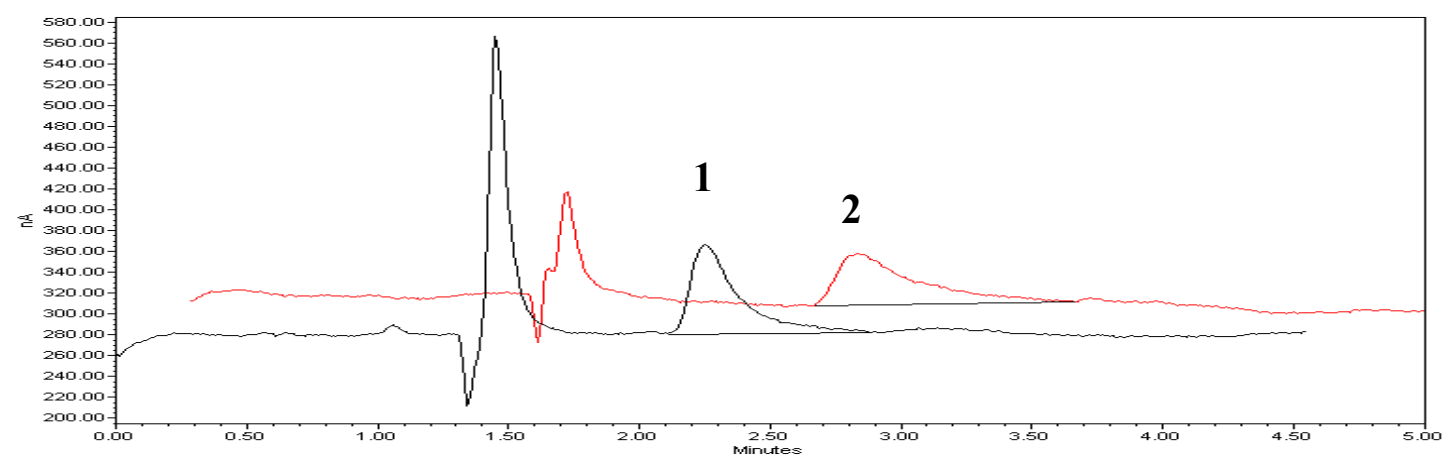

\section{b. Optimization of PAD settings}

The PAD settings which required optimization included spacer thickness, temperature control, and electrode potentials. The thickness of the spacer directly affected the linear velocity inside of the flow cell. Use of a thinner spacer resulted in a smaller cell volume, which led to a higher linear flow velocity. For a $3 \mathrm{~mm}$ working 
electrode, use of a $25 \mu \mathrm{m}$ spacer resulted in a flow cell volume of $0.15 \mu \mathrm{L}$, while use of a $50 \mu \mathrm{m}$ spacer resulted in a flow cell volume of $0.29 \mu \mathrm{L}$. The signal increased when a thinner spacer was used, while the noise remained constant. However, maximizing the signal-to-noise ratio by minimizing the flow cell volume had to be balanced against the need to select a mobile phase flow rate which was high enough to rapidly separate sample components. Use of a faster flow rate resulted in the need for a greater flow cell volume in order to avoid pressure build-up in the flow cell. For this method, a spacer of thickness of $50 \mu \mathrm{m}$ was ultimately selected in order to maintain proper electrode function at a flow rate of $1.00 \mathrm{~mL} / \mathrm{min}$.

The effect of temperature on the detection of hydrogen peroxide was explored. The Waters 2465 Electrochemical Detector was equipped with an oven that maintained a constant temperature for both the flow cell and the analytical columns. The oven temperature could be set between $15^{\circ} \mathrm{C}-45^{\circ} \mathrm{C}$. A temperature range of only $30^{\circ} \mathrm{C}-45^{\circ} \mathrm{C}$ was explored because it became readily apparent that the signal strength of a given sample of hydrogen peroxide increased as the temperature was raised. For this study, a standard solution of hydrogen peroxide was analyzed in triplicate. The optimal temperature for the detection of hydrogen peroxide was determined to be $45^{\circ} \mathrm{C}$ (the highest temperature achievable with the oven installed on the 2465 detector). Data from this study can be viewed in Table 6.1. There was no difference in retention time for hydrogen peroxide at this temperature vs. a cooler temperature. 
Table 6.1. Effect of temperature of the electrochemical detector flow cell on the peak area of hydrogen peroxide. The strongest signal for hydrogen peroxide was obtained at the highest oven setting available on the Waters 2465 Electrochemical Detector.

\begin{tabular}{|c|c|c|c|}
\hline Temperature $\left({ }^{\circ} \mathrm{C}\right)$ & Ave Peak Area & Stnd Dev Peak Area & $\%$ RSD Peak Area \\
\hline 30 & $1.90 \times 10^{4}$ & $1.50 \times 10^{3}$ & 7.8 \\
\hline 35 & $2.10 \times 10^{4}$ & $1.20 \times 10^{3}$ & 5.7 \\
\hline 40 & $2.50 \times 10^{4}$ & $3.60 \times 10^{2}$ & 1.4 \\
\hline 45 & $2.90 \times 10^{4}$ & $1.00 \times 10^{3}$ & 3.6 \\
\hline
\end{tabular}

In order to achieve optimal detection of hydrogen peroxide, optimization of the PAD settings was required. Because $E_{l}$ is the detection potential, the value of $E_{l}$ should have the greatest effect upon the signal strength of hydrogen peroxide. The values of $E_{2}$ and $E_{3}$ should have less of an effect, as they pertain to the cleaning and regeneration of the electrode surface, rather than to the detection of peroxide. The waveform was optimized by systematically varying $E_{l}$ while keeping the other parameters constant. The strongest signal for hydrogen peroxide was obtained with $E_{1}=+0.01 \mathrm{~V}, E_{2}=+0.65$, and $E_{3}=-0.65 \mathrm{~V}$ versus a palladium-hydrogen reference electrode. A similar waveform $\left(E_{1}=+0.05 \mathrm{~V}, E_{2}=+0.60 \mathrm{~V}, E_{3}=-0.60 \mathrm{~V}\right.$ vs. $\left.\mathrm{Ag} / \mathrm{AgCl}\right)$ has been demonstrated to provide long term peak area reproducibility, relative lack of sensitivity to dissolved oxygen, and reproducibility for early eluting peaks (71).

The initial experiments to optimize the electrode settings for pulsed amperometric detection were performed with the $150 \mathrm{mM} \mathrm{NaOH}$ mobile phase. After $150 \mathrm{mM} \mathrm{NaOAc}$ was added to the mobile phase, the optimization experiments were performed a second 
time to ensure that the correct settings were being applied. Samples of hydrogen peroxide at $0.5 \mathrm{ppm}(20 \mu \mathrm{L}$ injection volume $)$ were analyzed in duplicate. These data can be viewed in Table 6.2. Again, the strongest signal for hydrogen peroxide was obtained with $E_{1}=+0.01 \mathrm{~V}, E_{2}=+0.65$, and $E_{3}=-0.65 \mathrm{~V}$ versus a palladium-hydrogen reference electrode.The analysis of a hydrogen peroxide standard using this optimized method is presented in Figure 6.3.

Table 6.2. Effect of PAD settings on detection of a hydrogen peroxide standard. The greatest value for the peak area of hydrogen peroxide was obtained at $E_{l}=+0.01 \mathrm{~V}$, $E_{2}=+0.65 \mathrm{~V}$, and $E_{3}=-0.65 \mathrm{~V}$.

\begin{tabular}{|c|c|c|c|c|}
\hline$E_{1}(V)$ & $E_{2}(V)$ & $E_{3}(V)$ & Ave Peak Area & RSD Peak Area \\
\hline+0.15 & +0.65 & -0.65 & $6.80 \times 10^{3}$ & $1.3 \%$ \\
\hline+0.10 & +0.65 & -0.65 & $7.10 \times 10^{3}$ & $1.2 \%$ \\
\hline+0.05 & +0.65 & -0.65 & $7.30 \times 10^{3}$ & $1.8 \%$ \\
\hline+0.05 & +0.55 & -0.65 & $7.20 \times 10^{3}$ & $3.4 \%$ \\
\hline+0.05 & +0.55 & -0.55 & $7.30 \times 10^{3}$ & $1.8 \%$ \\
\hline+0.01 & +0.55 & -0.55 & $7.40 \times 10^{3}$ & $1.8 \%$ \\
\hline+0.01 & +0.65 & -0.65 & $7.70 \times 10^{3}$ & $2.3 \%$ \\
\hline+0.01 & +0.70 & -0.70 & $7.50 \times 10^{3}$ & $3.8 \%$ \\
\hline
\end{tabular}


Figure 6.3. Analysis of a $0.5 \mathrm{ppm}$ hydrogen peroxide standard by HPLC/ED in PAD mode. Conditions: Dionex CarboPac PA10 guard and analytical columns; isocratic elution with $150 \mathrm{mM} \mathrm{NaOH} / 150 \mathrm{mM} \mathrm{NaOAc}$; flow rate $1.0 \mathrm{~mL} / \mathrm{min} ; 20 \mu \mathrm{L}$ injection, $\mathrm{PAD}$ at $45^{\circ} \mathrm{C}\left(E_{1}=+0.01 \mathrm{~V}, t_{1}=300 \mathrm{~ms}, t_{s}=100 \mathrm{~ms}, E_{2}=+0.65 \mathrm{~V}, t_{2}=150 \mathrm{~ms}\right.$, $\left.E_{3}=-0.65 \mathrm{~V}, t_{3}=150 \mathrm{~ms}\right)$. Peak 1 , hydrogen peroxide, Rt $2.152 \mathrm{~min}$.

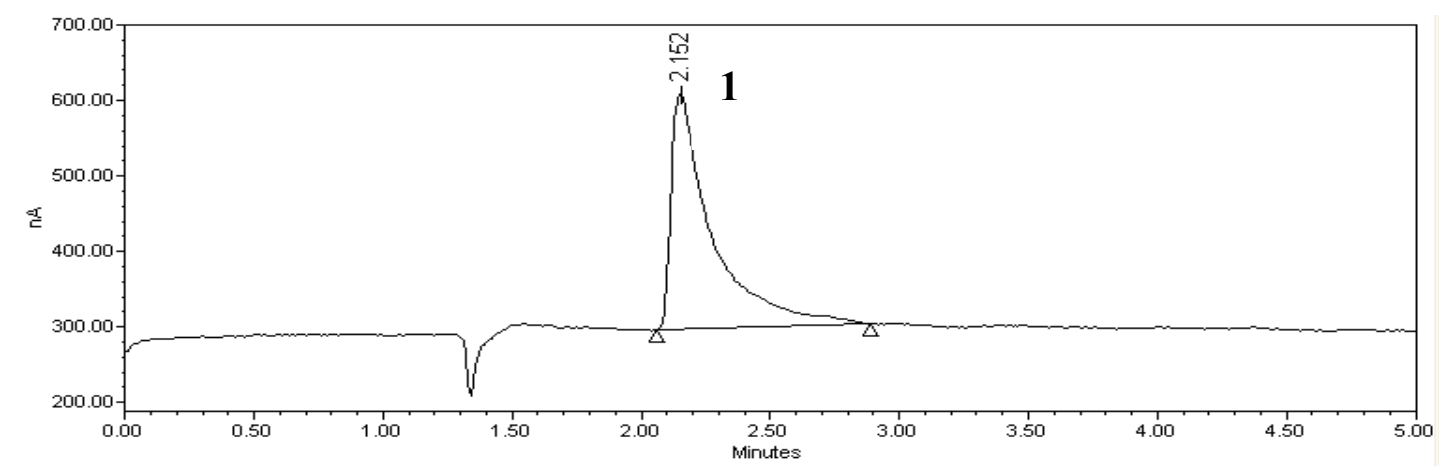

\section{Confirmation of hydrogen peroxide}

It was necessary to confirm the identity of the chromatographic peak which was believed to correspond to hydrogen peroxide. In order to do this, the flow cell was maintained in the off position so that the peroxide would not be destroyed by the working electrode. Three replicate $10 \mu \mathrm{L}$ injections of $300 \mathrm{ppm}$ hydrogen peroxide were performed. A total of $3 \mu \mathrm{g}$ hydrogen peroxide was injected each time. Beginning at the time of sample injection, eluent fractions were collected in one minute intervals from the until five minutes after the injection. Because the test strips required a $\mathrm{pH}$ of $1-12$ to function properly, the collected fractions were treated with $1 \mathrm{M} \mathrm{HCl}$ to bring the $\mathrm{pH}$ below 12. Liquid from each fraction was pipetted onto the surface of a commercial hydrogen peroxide test strip. The test was based on the colorimetric reaction of peroxide with 2,2'-azino-bis(3-ethylbenzothiazoline)-6-sulfonate; the presence of a blue color on 
the test strip was indicative of the presence of peroxide. This test is not specific to hydrogen peroxide as both organic and inorganic peroxides in aqueous solution and organic solvents can be measured. According to the manufacturer, results were semiquantitative over the range of $0.5 \mu \mathrm{g} / \mathrm{mL}-25.0 \mu \mathrm{g} / \mathrm{mL}$.

For all three trials, only the eluent fractions which contained hydrogen peroxide yielded a positive result on the test strips. Each of the positive fractions corresponded to the time interval of minute two to minute three post-injection when hydrogen peroxide was believed to elute. Given that $3 \mu \mathrm{g}$ of hydrogen peroxide were injected, and that each fraction collected contained approximately $1 \mathrm{~mL}$ of eluent $(1 \mathrm{~mL} / \mathrm{min} * 1 \mathrm{~min}=1 \mathrm{~mL})$, then the peroxide-containing fractions should have contained approximately $3 \mu \mathrm{g} / \mathrm{mL}$ hydrogen peroxide. However, the color on the test strips corresponded to the $0.5 \mu \mathrm{g} / \mathrm{mL}$ color on the test kit, indicating that the results from this kit are indeed only semiquantitative. Nonetheless, these results confirm that the peak which elutes at 2.15 minutes is hydrogen peroxide.

\section{Development of $150 \mathrm{mM}$ sodium acetate mobile phase with electrochemical} detection in DC mode for the detection of post-blast hydrogen peroxide residues

\section{a. Optimization of mobile phase}

A new mobile phase with a different detection mode were developed after it was determined that the original method of $150 \mathrm{mM} \mathrm{NaOH} / 150 \mathrm{mM} \mathrm{NaOAc}$ with PAD mode was unsuitable for the analysis of post-blast samples due to problems with interfering compounds in field samples, along with the presence of high background and tailing of the peroxide peak (see Figure 6.3). A series of scanning voltammograms were 
constructed across the range of $E=0 \mathrm{mV}$ to $E=+750 \mathrm{mV}$. It was determined that detection of hydrogen peroxide was enhanced when a higher detection potential was employed. However, at too high of a detection potential (greater than $E=+500 \mathrm{mV}$ ), the oxidation of sodium hydroxide in the mobile phase overwhelmed the peroxide signal. When a mobile phase was employed that included sodium acetate without sodium hydroxide, the hydrogen peroxide signal was no longer masked at higher detection potentials. Mobile phase compositions of $5.0 \mathrm{mM} \mathrm{NaOAc}, 50 \mathrm{mM} \mathrm{NaOAc}, 150 \mathrm{mM}$ $\mathrm{NaOAc}$, and $300 \mathrm{mM} \mathrm{NaOAc}$ were tested with the CarboPac PA 10 columns. These data are presented in Table 6.3. Hydrogen peroxide eluted at approximately 1.90 minutes for all four mobile phase compositions. Peak response was notably lower for the $5.0 \mathrm{mM}$ $\mathrm{NaOAc}$ mobile phase compared to the other three compositions, presumably because the 5.0 mM NaOAc mobile phase was too dilute to conduct current through the flow cell (157). The combination of the $150 \mathrm{mM} \mathrm{NaOAc}$ mobile phase at $\mathrm{pH} 10.5$ with the PA10 column resulted in the greatest peak area and peak symmetry for the hydrogen peroxide standard, with baseline resolution of the peroxide peak from the water peak.

It should be noted that this combination of mobile phase and analytical column resulted in the inability to detect the carbohydrate standards. Glucose, sucrose, and fructose did not elute from the column up to thirty minutes following injection. A gradient procedure with $150 \mathrm{mM} \mathrm{NaOAc}$ and $150 \mathrm{mM} \mathrm{NaOH}$ was briefly explored. Because inclusion of even $2 \% 150 \mathrm{mM} \mathrm{NaOH}$ in the mobile phase dramatically increased the background signal without resulting in elution of the carbohydrates, this procedure was not explored further. As the priority for this method was the separation and detection 
of hydrogen peroxide, further optimization to permit separation and detection of the sugars was not performed.

Table 6.3. Effect of new mobile phase concentration on retention time and peak area of hydrogen peroxide. The combination of $150 \mathrm{mM} \mathrm{NaOAc}$ with the PA10 column yielded the maximum signal for hydrogen peroxide and baseline resolution of the peroxide peak from the water peak. This mobile phase was selected to undergo further optimization.

\begin{tabular}{|c|c|c|c|c|}
\hline Mobile Phase & {$[\mathrm{HP}]$} & $R t$ & $\begin{array}{c}\text { Average } \\
\text { Peak Area }\end{array}$ & $\begin{array}{c}\text { \& RSD } \\
\text { Peak Area }\end{array}$ \\
\hline $5 \mathrm{mM} \mathrm{NaOAc}$ & $10 \mathrm{ppm}$ & $1.992 \mathrm{~min}$ & $5.40 \times 10^{3}$ & 12 \\
\hline $50 \mathrm{mM} \mathrm{NaOAc}$ & $0.5 \mathrm{ppm}$ & $1.885 \mathrm{~min}$ & $1.00 \times 10^{4}$ & 4.0 \\
\hline $150 \mathrm{mM} \mathrm{NaOAc}$ & $0.5 \mathrm{ppm}$ & $1.896 \mathrm{~min}$ & $1.03 \times 10^{4}$ & 0.7 \\
\hline $300 \mathrm{mM} \mathrm{NaOAc}$ & $0.5 \mathrm{ppm}$ & $1.891 \mathrm{~min}$ & $9.10 \times 10^{3}$ & 2.6 \\
\hline
\end{tabular}

\section{b. Optimization of DC mode}

A theoretical hydrodynamic voltammogram for the oxidation of hydrogen peroxide is presented in Figure 6.4. This type of graph is used to determine the optimal detection potential for an analyte of interest. In the zero current region, the applied potential is insufficient to force oxidation of the analyte to occur. In the intermediate region, the peak height is rising with increasing potential. The applied potential controls the kinetics of the heterogeneous electron transfer from the analyte to the surface of the electrode. In the plateau region, peak height is independent of the applied potential. The rate determining factor is the diffusion of sample to the electrode surface, meaning that 
the current is proportional to the rate of transport of molecules per unit surface area and per unit time. The optimal detection potential lies on the plateau region within 50-100 $\mathrm{mV}$ of the break in the curve. This potential will offer selectivity to the analyte of interest with minimal background current and fewer interferences than a higher potential (157). In the second region of rising peak height with increasing potential, any water which is present is undergoing electrolysis. This results in the generation of high levels of background signal which can mask the signal of the analyte.

Figure 6.4. Theoretical hydrodynamic voltammogram for hydrogen peroxide.

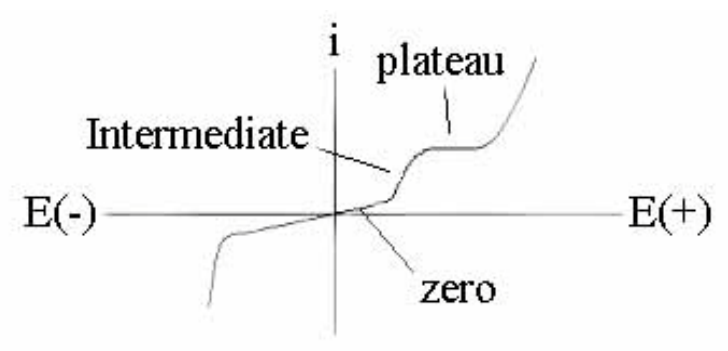

A hydrodynamic voltammogram was constructed for $0.5 \mathrm{ppm}$ hydrogen peroxide in $150 \mathrm{mM} \mathrm{NaOAc}$ at $\mathrm{pH} 10.5$ across the range of $E=0 \mathrm{mV}$ to $E=+800 \mathrm{mV}$ to determine the optimal detection potential. This graph is presented in Figure 6.5. The optimal detection potential is the point where the graph just begins to plateau, in this case, at $E=+400 \mathrm{mV}$. At potentials lower than $+400 \mathrm{mV}$, the signal strength of hydrogen peroxide is weaker, the peak height is unstable, and the limit of detection will be lower. At potentials higher than $+400 \mathrm{mV}$, there is a greater chance that additional, interfering species will be oxidized that could potentially mask the signal from hydrogen peroxide. This is important because this method is intended to be applied to the analysis of post- 
blast samples which are notorious for containing matrix compounds and other interfering materials. Therefore, a detection potential was selected that was the lowest potential required to fully oxidize hydrogen peroxide, while avoiding the problem of oxidizing additional compounds with a higher $\mathrm{E}_{0}$. Another problem to avoid was the presence of increased levels of background signal as a result of the oxidation of water and sodium hydroxide in the mobile phase. This effect was reduced when $E=+400 \mathrm{~V}$ (in comparison to $E=+800 \mathrm{~V}$ ). A chromatogram of a hydrogen peroxide standard analyzed using this optimized method is presented in Figure 6.6.

Figure 6.5. Hydrodynamic voltammogram of hydrogen peroxide in $150 \mathrm{mM} \mathrm{NaOAc}$. The optimal detection potential for hydrogen peroxide is $E=+400 \mathrm{mV}$.

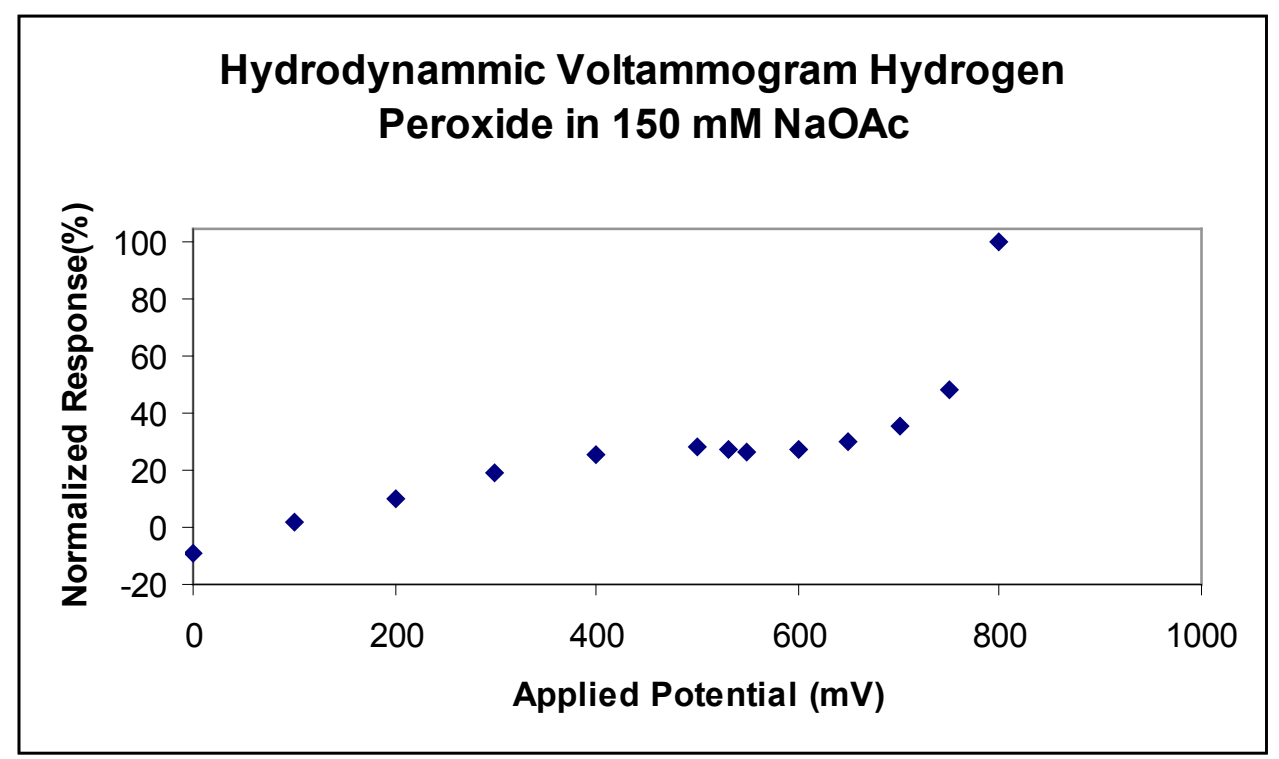


Figure 6.6. Analysis of $0.5 \mathrm{ppm}$ hydrogen peroxide by HPLC/ED in DC mode.

Conditions: Dionex CarboPac PA10 guard and analytical columns; isocratic elution with $150 \mathrm{mM} \mathrm{NaOAc}$ at $\mathrm{pH} 10.5$; flow rate $1.00 \mathrm{~mL} / \mathrm{min} ; 20 \mu \mathrm{L}$ injection, $\mathrm{T}=45^{\circ} \mathrm{C}, \mathrm{DC}$ mode with $\mathrm{E}=+400 \mathrm{mV}$. Peak 1, hydrogen peroxide, Rt $1.897 \mathrm{~min}$. Under these conditions, the hydrogen peroxide peak displays minimal tailing, and baseline resolution from the water peak is achieved.

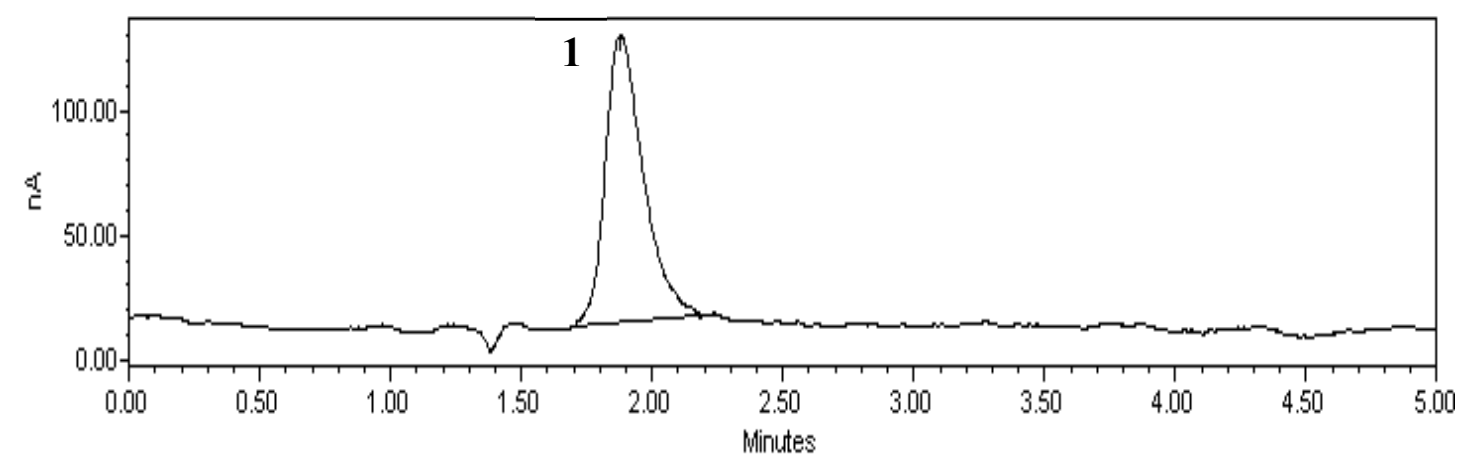

The symmetry of the hydrogen peroxide peak on the PA10 column with the 150 $\mathrm{mM} \mathrm{NaOAc}$ mobile phase at $\mathrm{pH} 10.5$ was calculated to be 0.71 . While this is still not an ideal, Gaussian type-shape with a symmetry value equal to 1.00 , this is nonetheless an improvement over the peak symmetry value of 0.39 which was obtained for hydrogen peroxide when the $150 \mathrm{mM} \mathrm{NaOH}$ mobile phase was used with the PA1 column.

\section{Re-confirmation of hydrogen peroxide}

It was necessary to confirm the identity of the chromatographic peak which was believed to correspond to hydrogen peroxide. In order to do this, the flow cell was maintained in the off position so that the peroxide would not be destroyed by the working electrode. Three replicate $10 \mu \mathrm{L}$ injections of $300 \mathrm{ppm}$ hydrogen peroxide were 
performed. A total of $3 \mu \mathrm{g}$ hydrogen peroxide was injected each time. Beginning at the time of sample injection, eluent fractions were collected in one minute intervals from the time of injection until five minutes after injection. Liquid from each fraction was pipetted onto the surface of a commercial hydrogen peroxide test strip. The test was based on the colorimetric reaction of peroxide with 2,2'-azino-bis(3-ethylbenzothiazoline)-6-sulfonate; the presence of a blue color on the test strip was indicative of the presence of peroxide. This test is not specific to hydrogen peroxide as both organic and inorganic peroxides in aqueous solution and organic solvents can be measured. According to the manufacturer, results were semi-quantitative over the range of $0.50 \mu \mathrm{g} / \mathrm{mL}-25.0 \mu \mathrm{g} / \mathrm{mL}$.

For all three trials, only the eluent fractions which contained hydrogen peroxide yielded a positive result on the test strips. Each of the positive fractions corresponded to the time interval of 1.5 minutes to 2.5 minutes post-injection when hydrogen peroxide was thought to elute. Given that $3 \mu \mathrm{g}$ of hydrogen peroxide were injected, and that each fraction collected contained approximately $1 \mathrm{~mL}$ of eluent $(1 \mathrm{~mL} / \mathrm{min} * 1 \mathrm{~min}=1 \mathrm{~mL})$, then the peroxide-containing fractions should have contained approximately $3 \mu \mathrm{g} / \mathrm{mL}$ hydrogen peroxide. However, the color on the test strips corresponded to the $5 \mu \mathrm{g} / \mathrm{mL}$ color on the test kit, indicating that the results from this kit are indeed only semiquantitative. Nonetheless, these results confirm that the peak which elutes at 1.90 minutes is hydrogen peroxide.

\section{Summary}

After testing four analytical columns, six mobile phase compositions, and two modes of electrochemical detection, two methods for the analysis of hydrogen peroxide 
were developed. The first method utilized a CarboPac PA10 column with a $150 \mathrm{mM}$ $\mathrm{NaOH}$ and $150 \mathrm{mM} \mathrm{NaOAc}$ mobile phase with electrochemical detection in PAD mode. The retention time of hydrogen peroxide was 2.152 minutes, but the symmetry of the peak shape was poor. This method also proved unsuitable for the analysis of post-blast hydrogen peroxide residues, as will be discussed in Chapter 7. By changing from a mobile phase which was $150 \mathrm{mM} \mathrm{NaOH}$ and $150 \mathrm{mM} \mathrm{NaOAc}$ to a mobile phase which contained $150 \mathrm{mM} \mathrm{NaOAc}$ at $\mathrm{pH} 10.5$, the retention time of hydrogen peroxide was reduced to 1.90 minutes and the shape of the peroxide peak was improved. Changing the mode of detection from PAD mode to DC mode served to make this method more suitable for the analysis of post-blast hydrogen peroxide residues. This data will be shown in Chapter 7.

\section{Development of HPLC/FD Method}

\section{Selection of analytical column}

A C-18 analytical column was selected for the separation of hydrogen peroxide because Kok et al. (143), Francois et al. (141), and Qi et al. (144) each utilized a C-18 analytical column for the separation of hydrogen peroxide and organic peroxides. The composition of the mobile phase was taken from Kok et al. (143) and consisted of 1.00 $\mathrm{mM} \mathrm{H}_{2} \mathrm{SO}_{4}$ with $0.10 \mathrm{mM}$ EDTA. Two additional analytical columns were also explored for the separation of hydrogen peroxide in conjunction with the sulfuric acid mobile phase: a C-8 column (4.6 x $150 \mathrm{~mm})$, and a CarboPac PA1 (4.6 x $250 \mathrm{~mm})$ column. Figure 6.7 demonstrates the peak shapes obtained with the different columns. 
Figure 6.7. Analysis of 0.5 ppm hydrogen peroxide by HPLC/FD. Conditions: $20 \mu 1$ injection volume, $1.00 \mathrm{mM} \mathrm{H}_{2} \mathrm{SO}_{4}$ and $0.10 \mathrm{mM}$ EDTA mobile phase. (1) $\mathrm{H}_{2} \mathrm{O}_{2}$ on a C-8 column, Rt 3.452 min; (2) $\mathrm{H}_{2} \mathrm{O}_{2}$ on a PAl column, Rt 4.163 min; (3) $\mathrm{H}_{2} \mathrm{O}_{2}$ on a C-18 column, Rt $5.074 \mathrm{~min}$.

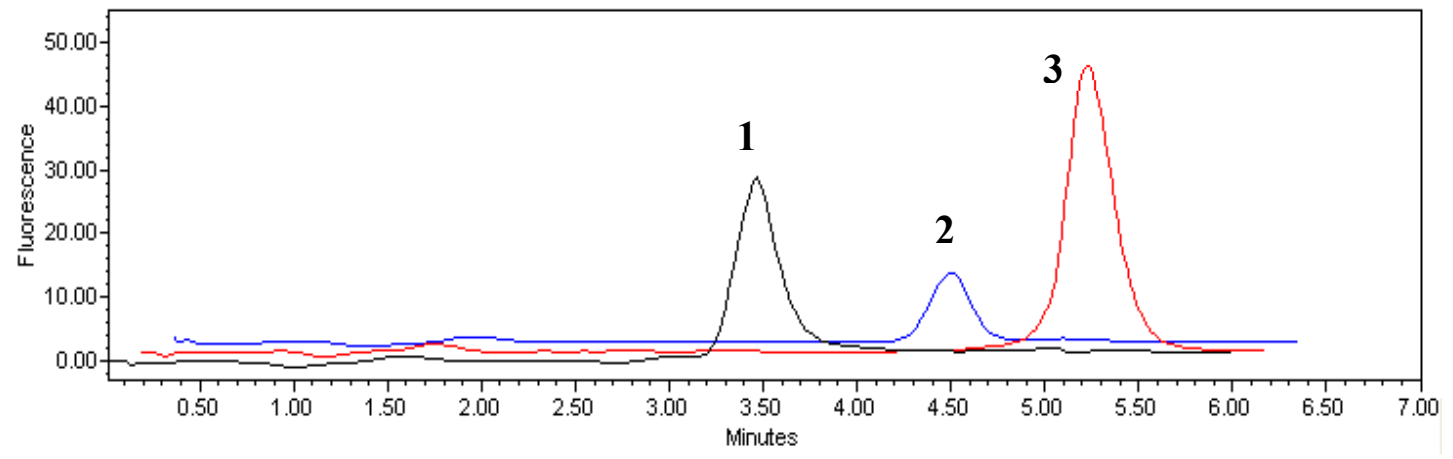

Table 6.4. Effect of analytical column on retention time and peak area of $0.5 \mathrm{ppm}$ hydrogen peroxide. A mobile phase which was $1.00 \mathrm{mM} \mathrm{H}_{2} \mathrm{SO}_{4}$ and $0.10 \mathrm{mM}$ EDTA was used with all three columns. The C-18 column offers superior retention of hydrogen peroxide in comparison to the other two columns.

\begin{tabular}{|c|c|c|c|c|c|}
\hline Column & Dimensions & Ave Peak & RSD Peak & Ave Rt & RSD Rt \\
& & Area & Area & & \\
\hline C-18 & $4.6 \mathrm{~mm} \times 250 \mathrm{~mm}$ & $8.41 \times 10^{5}$ & $0.96 \%$ & $5.07 \mathrm{~min}$ & $0.43 \%$ \\
\hline C-8 & $4.6 \mathrm{~mm} \times 150 \mathrm{~mm}$ & $4.99 \times 10^{5}$ & $0.11 \%$ & $3.45 \mathrm{~min}$ & $0.59 \%$ \\
\hline PA1 & $4.6 \mathrm{~mm} \times 250 \mathrm{~mm}$ & $2.17 \times 10^{5}$ & $1.3 \%$ & $4.16 \mathrm{~min}$ & $0.24 \%$ \\
\hline
\end{tabular}

The retention time and peak area for hydrogen peroxide with each column are presented in Table 6.4. All three columns were able to resolve hydrogen peroxide, but the C-18 column facilitated the greatest retention of the compound, as well as the greatest 
peak area. Therefore, the C-18 column was utilized in all further experiments involving method development and sample analysis.

\section{Development of Optimized Mobile Phase and Flow Rate}

The initial separation of hydrogen peroxide was conducted using a mobile phase which consisted of $1.00 \mathrm{mM} \mathrm{H}_{2} \mathrm{SO}_{4}$ and $0.10 \mathrm{mM}$ EDTA. Two additional mobile phase compositions were tested to determine if the addition of more acid to the mobile phase would affect retention time or peak area: $2.00 \mathrm{mM} \mathrm{H}_{2} \mathrm{SO}_{4}$ with $0.10 \mathrm{mM}$ EDTA; and 1.50 $\mathrm{mM} \mathrm{H}_{2} \mathrm{SO}_{4}$ with $0.10 \mathrm{mM}$ EDTA. Use of the second two mobile phase compositions did not result in a different retention time or level of detector response for hydrogen peroxide from the $1 \mathrm{mM} \mathrm{H}_{2} \mathrm{SO}_{4}$ mobile phase. Therefore, they were not explored further.

An optimized flow rate was determined using a mobile phase which consisted of $1.00 \mathrm{mM} \mathrm{H}_{2} \mathrm{SO}_{4}$ and $0.10 \mathrm{mM}$ EDTA. For this study, the flow rate of the reagent ( $8 \mu \mathrm{M}$ hemin and $80 \mu \mathrm{M}$ POPHA in ammonia buffer at $\mathrm{pH} 9.5$ ) was held at $0.20 \mathrm{~mL} / \mathrm{min}$ and the flow rate of the base ( $29 \%$ ammonium hydroxide) was held at $0.25 \mathrm{~mL} / \mathrm{min}$. The effect of changing flow rate of the mobile phase on the detection of the fluorescent dimer formed from the reaction of hydrogen peroxide with the reagent solution was measured. These data are available in Table 6.5. It was determined that $0.60 \mathrm{~mL} / \mathrm{min}$ was the optimal flow rate for this configuration, as it allowed for both maximal detection of the POPHA dimer and a relatively quick separation of hydrogen peroxide. Increasing the flow rate to $0.70 \mathrm{~mL} / \mathrm{min}$ resulted in reduced response of the POPHA dimer, but a more rapid separation of hydrogen peroxide. Decreasing the flow rate to $0.50 \mathrm{~mL} / \mathrm{min}$ resulted 
in a slightly decreased response for the POPHA dimer, but a longer retention time for hydrogen peroxide.

Table 6.5. Effect of mobile phase flow rate on peak area and retention time of $0.5 \mathrm{ppm}$ hydrogen peroxide. Conditions: $\mathrm{C}-18$ column, mobile phase $1 \mathrm{mM} \mathrm{H}_{2} \mathrm{SO}_{4}$ and $0.1 \mathrm{mM}$ EDTA. The greatest average peak area was obtained with a $0.6 \mathrm{~mL} / \mathrm{min}$ flow rate.

\begin{tabular}{|c|c|c|c|c|}
\hline Vm & Ave Peak Area & \% RSD Peak Area & Ave Rt & \% RSD Rt \\
\hline $0.5 \mathrm{~mL} / \mathrm{min}$ & $4.86 \times 10^{5}$ & 1.3 & $6.00 \mathrm{~min}$ & 0.2 \\
\hline $0.6 \mathrm{~mL} / \mathrm{min}$ & $5.02 \times 10^{5}$ & 2.0 & $5.08 \mathrm{~min}$ & 0.1 \\
\hline $0.7 \mathrm{~mL} / \mathrm{min}$ & $4.18 \times 10^{5}$ & 0.7 & $4.43 \mathrm{~min}$ & 0.1 \\
\hline
\end{tabular}

The addition of organic solvent to the mobile phase is necessary for the separation of organic peroxides in a mixture with hydrogen peroxide. Therefore, the effect of adding organic solvent to the mobile phase on the signal strength of hydrogen peroxide was explored. Results from the incorporation of acetonitrile in the mobile phase are presented in Table 6.6. It is clear that by increasing the percentage of acetonitrile in the mobile phase, the signal strength of hydrogen peroxide decreased. It is theorized that acetonitrile was interacting negatively with either the hemin catalyst or the $p$-hydroxyphenylacetic acid, leading to a reduction in production of the dimer. Therefore, no organic solvent was added to the mobile phase in order to keep the signal strength of hydrogen peroxide as high as possible to achieve a low limit of detection. 
Table 6.6. Effect of acetonitrile on detection of $0.5 \mathrm{ppm}$ hydrogen peroxide. Conditions: C-18 column, $1.00 \mathrm{mM} \mathrm{H}_{2} \mathrm{SO}_{4}$ and $0.10 \mathrm{mM}$ EDTA aqueous component of the mobile phase. The addition of organic phase is necessary for the separation of organic peroxides, but negatively impacts the detection of hydrogen peroxide.

\begin{tabular}{|c|c|c|}
\hline Percentage of MeCN & Ave Peak Area & $\%$ RSD Peak Area \\
\hline $0 \%$ & $2.23 \times 10^{6}$ & 1.3 \\
\hline $5 \%$ & $5.65 \times 10^{5}$ & 0.6 \\
\hline $7 \%$ & $4.62 \times 10^{5}$ & 3.1 \\
\hline $10 \%$ & $3.30 \times 10^{5}$ & 8.3 \\
\hline $15 \%$ & $2.40 \times 10^{5}$ & \\
\hline
\end{tabular}

\section{Optimization of reaction tubing length and temperature}

The composition of the reagent solution was taken from the work of Qi et al.

(144). This paper studied the effects of replacing horseradish peroxidase with hemin as the reaction catalyst. It was found that the products obtained using the two catalysts were spectrally indistinguishable because the same compound is formed. Furthermore, the limit of detection for hydrogen peroxide using both enzymes was comparable. The kinetic profile for the rate of fluorescence development with hemin and horseradish peroxidase had no distinct differences. The use of both enzymes resulted in a maximization of fluorescence signal within twenty seconds. Because hemin required less stringent experimental conditions than horseradish peroxidase, development of the fluorescence method continued with hemin. By independently varying the concentrations of $p$-hydroxyphenylacetic acid and hemin in the reagent solution, Qi et al. (144) 
determined that the optimal conditions to maximize fluorescence intensity were $8 \mu \mathrm{M}$ hemin and $80 \mu \mathrm{M}$-hydroxyphenylacetic acid in ammonia buffer at $\mathrm{pH} 10$. This reagent solution was utilized in the HPLC/FD method for hydrogen peroxide analysis. A Waters 515 pump was used to deliver the reagent solution at an optimized flow rate of 0.20 $\mathrm{mL} / \mathrm{min}$.

Four sets of reaction tubing of different lengths and internal diameters were tested to determine which would permit maximum conversion of POPHA to the fluorescent dimer while also resulting in minimal peak broadening. The average peak area, retention time, and residence time in the reaction tubing for the same $0.5 \mathrm{ppm}$ hydrogen peroxide standard with each set of tubing is presented in Table 6.7. The residence time of the reactants in the reaction tubing was determined using a two step process. First, the volume of each set of tubing was calculated using equation 1 , where $r$ is the radius of the tubing and $l$ is the length of the tubing.

$$
\begin{aligned}
& \text { Volume of a cylinder }=\pi r^{2} l \\
& \text { Volume }=\pi(0.025 \mathrm{~cm})^{2} * 200 \mathrm{~cm}=0.393 \mathrm{~cm}^{3}
\end{aligned}
$$

The combined flow rate from the mobile phase pump and the reagent pump was $0.80 \mathrm{~mL} / \mathrm{min}$. If $1.00 \mathrm{~mL}=1 \mathrm{~cm}^{3}$, then $0.80 \mathrm{~mL} / \mathrm{min}=0.8 \mathrm{~cm}^{3} / \mathrm{min}$. The time taken for a slug of solution to fill the volume in the reaction tubing is given in equation 2.

$$
\begin{aligned}
& \text { Distance / velocity }=\text { time } \\
& 0.393 \mathrm{~cm}^{3} / 0.8 \mathrm{~cm}^{3} \min ^{-1}=0.491 \mathrm{~min} \\
& 0.491 \mathrm{~min} * 60 \mathrm{~s} \mathrm{~min}^{-1}=29.5 \mathrm{~s}
\end{aligned}
$$


Table 6.7. Effect of length and inner diameter of reaction tubing on detection of $0.5 \mathrm{ppm}$ hydrogen peroxide. Conditions: C-18 column, $1.00 \mathrm{mM} \mathrm{H}_{2} \mathrm{SO}_{4}$ / $0.10 \mathrm{mM}$ EDTA mobile phase at $0.60 \mathrm{~mL} / \mathrm{min}$. Optimal detection of hydrogen peroxide was achieved when the residence time of the reagents in the tubing was at least $20 \mathrm{~s}$ to yield the POPHA dimer.

\begin{tabular}{|c|c|c|c|c|c|c|}
\hline $\begin{array}{c}\text { Length } \\
(\mathrm{m})\end{array}$ & $\begin{array}{c}\text { ID } \\
(\mathrm{mm})\end{array}$ & Ave Area & $\begin{array}{c}\% \text { RSD } \\
\text { Area }\end{array}$ & $\begin{array}{c}\text { Ave Rt } \\
(\mathrm{min})\end{array}$ & $\begin{array}{c}\% R S D \\
R t\end{array}$ & $\begin{array}{c}\text { Residence } \\
\text { Time }\end{array}$ \\
\hline 1.6 & 0.25 & $4.90 \times 10^{4}$ & 17 & 4.74 & 0.01 & $5.89 \mathrm{~s}$ \\
\hline 1.6 & 0.50 & $2.33 \times 10^{5}$ & 3.3 & 5.05 & 0.07 & $23.6 \mathrm{~s}$ \\
\hline 2.0 & 0.50 & $5.16 \times 10^{5}$ & 4.9 & 5.16 & 0.01 & $29.4 \mathrm{~s}$ \\
\hline 5.0 & 0.50 & $4.66 \times 10^{5}$ & 7.2 & 6.05 & 0.08 & $73.8 \mathrm{~s}$ \\
\hline
\end{tabular}

The reaction tubing which was 1.6 meters in length by $0.25 \mathrm{~mm}$ internal diameter resulted in the smallest signal for a standard solution of $0.5 \mathrm{ppm}$ hydrogen peroxide. The residence time of the reactants in this set of tubing is only $5.89 \mathrm{~s}$, meaning that the fluorescence intensity is below the possible maximum value predicted to occur at the $20 \mathrm{~s}$ mark by Qi et al. (144). This tubing was too short and too narrow for the reaction of hydrogen peroxide with POPHA and hemin to produce the full complement of the fluorescent dimer. The reaction tubing which was 1.6 meters in length by $0.50 \mathrm{~mm}$ internal diameter resulted in a four-fold increase in signal intensity over the tubing of $0.25 \mathrm{~mm}$ internal diameter. This is to be expected, given the dependence upon $r^{2}$.

Doubling the radius will result in a four-fold increase in volume, which in turn will result in a four-fold increase in residence time. The reaction tubing which was 2.0 meters in length by $0.50 \mathrm{~mm}$ internal diameter resulted in the greatest peak area for the standard 
solution of $0.5 \mathrm{ppm}$ hydrogen peroxide. The residence time for this tubing was $29.4 \mathrm{~s}$, which is above the reaction maximum of $20 \mathrm{~s}$. A second benefit to using this tubing for the post-column derivatization reaction was that it was the only set of tubing which could be contained within a temperature-controlled housing which was available for use in this experiment. This permitted better control of the derivatization reaction because there was less of an effect from fluctuations in room temperature.

Additional experiments were conducted to optimize the temperature of the reaction tubing in order to maximize the signal strength of the fluorescent dimer. These data are presented in Table 6.8. The greatest peak area for the fluorescent dimer was obtained at $25^{\circ} \mathrm{C}$, which was the lowest setting for the reactor housing. Data collected at $25^{\circ} \mathrm{C}$ also had the highest RSD value for this set of experiments. It is clear from Table 6.8 that signal response decreased as temperature increased. Previous researchers had maintained just the analytical column at $1^{\circ} \mathrm{C}(144)$, or the column, the reagent solution, the mobile phase, and the buffer at $1^{\circ} \mathrm{C}(141)$ for the stated purpose of stabilizing the organic peroxides. To explore the effects of reagent temperature on peak area for hydrogen peroxide, experiments were conducted in which the reagent solution was maintained in an ice bath. Minimal differences were noted in peak area and retention time for hydrogen peroxide when the reagent solution was maintained at $4^{\circ} \mathrm{C}$, versus experiments conducted with the reagent solution maintained either at $28^{\circ} \mathrm{C}$ or at $60^{\circ} \mathrm{C}$. It was therefore determined to be unnecessary to cool the reagent solution. Because separation of hydrogen peroxide with a low limit of detection was obtained when the column was maintained at room temperature, no efforts were made to cool the column. 
Table 6.8. Effect of temperature of the reaction tubing on detection of 0.5 ppm hydrogen peroxide. Conditions: C-18 column, $1.00 \mathrm{mM} \mathrm{H}_{2} \mathrm{SO}_{4}$ / $0.10 \mathrm{mM}$ EDTA mobile phase at $0.60 \mathrm{~mL} / \mathrm{min}$. Maintaining the reaction tubing at a lower temperature increased production of the POPHA dimer.

\begin{tabular}{|c|c|c|}
\hline Temperature & Ave Peak Area & $\%$ RSD Peak Area \\
\hline $25^{\circ} \mathrm{C}$ & $3.57 \times 10^{5}$ & 5.9 \\
\hline $30^{\circ} \mathrm{C}$ & $2.06 \times 10^{5}$ & 1.8 \\
\hline $40^{\circ} \mathrm{C}$ & $1.73 \times 10^{5}$ & 2.0 \\
\hline $50^{\circ} \mathrm{C}$ & $1.48 \times 10^{5}$ & 1.9 \\
\hline
\end{tabular}

\section{Optimization of base solution}

In accordance with previous studies a $29 \%$ solution of ammonium hydroxide was initially used to raise the $\mathrm{pH}$ of the reaction solution above 9.5 to maximize detection of the POPHA dimer (141) (143) (144). This solution was initially delivered by syringe pump through either plastic disposable syringes or glass gas-tight syringes equipped with a metal needle. While use of this base solution with either type of syringe did achieve the desired results, such a concentrated solution of base corroded the metal needles attached to the syringe. In order to preserve needle function, a dilute solution of sodium hydroxide was used in place of the concentrated ammonium hydroxide. As shown in Table 6.9, a solution of $50 \mathrm{mM} \mathrm{NaOH}$ and $100 \mathrm{mM} \mathrm{NaOH}$ resulted in a greater detector response for the POPHA dimer than did $150 \mathrm{mM} \mathrm{NaOH}$. Results for $100 \mathrm{mM} \mathrm{NaOH}$ and $50 \mathrm{mM}$ $\mathrm{NaOH}$ were very similar, although samples analyzed with the $50 \mathrm{mM} \mathrm{NaOH}$ had a lower RSD value. Nonetheless, it was decided that $100 \mathrm{mM} \mathrm{NaOH}$ would be used in all further 
experiments. Ultimately, the syringe pump was replaced with an additional Waters 515 pump, and base was delivered at a rate of $0.20 \mathrm{ml} / \mathrm{min}$.

Table 6.9. Effect of concentration of sodium hydroxide on detection of $0.5 \mathrm{ppm}$ hydrogen peroxide. Conditions: C-18 column, $1.00 \mathrm{mM} \mathrm{H}_{2} \mathrm{SO}_{4}$ / $0.10 \mathrm{mM}$ EDTA mobile phase at $0.60 \mathrm{~mL} / \mathrm{min} ; \mathrm{V}_{\text {base }} 0.25 \mathrm{~mL} / \mathrm{min}$. Despite a higher \%RSD value, $100 \mathrm{mM} \mathrm{NaOH}$ was selected as the base solution.

\begin{tabular}{|c|c|c|}
\hline$[\mathrm{NaOH}] \mathrm{mol} / \mathrm{L}$ & Ave Peak Area & \% RSD Peak Area \\
\hline 0.05 & $2.14 \times 10^{5}$ & 0.3 \\
\hline 0.10 & $2.14 \times 10^{5}$ & 1.9 \\
\hline 0.15 & $2.00 \times 10^{5}$ & 2.5 \\
\hline
\end{tabular}

\section{Optimization of Fluorescence Detector Settings}

A series of excitation and emission wavelengths were investigated to determine the combination of wavelengths which would maximize detection of the POPHA dimer. Samples of hydrogen peroxide at $0.5 \mathrm{ppm}$ were analyzed in duplicate by varying first the emission wavelength and second the excitation wavelength. These data can be seen in Figures 6.8 and 6.9. The most promising wavelengths from each group were selected, and these pairs were tested further (see Table 6.10). It was determined that detection of the POPHA dimer was maximized when an excitation wavelength of $320 \mathrm{~nm}$ was paired with an emission wavelength of $405 \mathrm{~nm}$. This set of wavelengths is similar to what has been employed in previous studies (143) (145) (158). 
Figure 6.8. Effect of changing emission wavelength on detection of hydrogen peroxide.

$\lambda_{\text {excitation }}=330 \mathrm{~nm}$; an emission maximum for the POPHA dimer is present at $405 \mathrm{~nm}$.

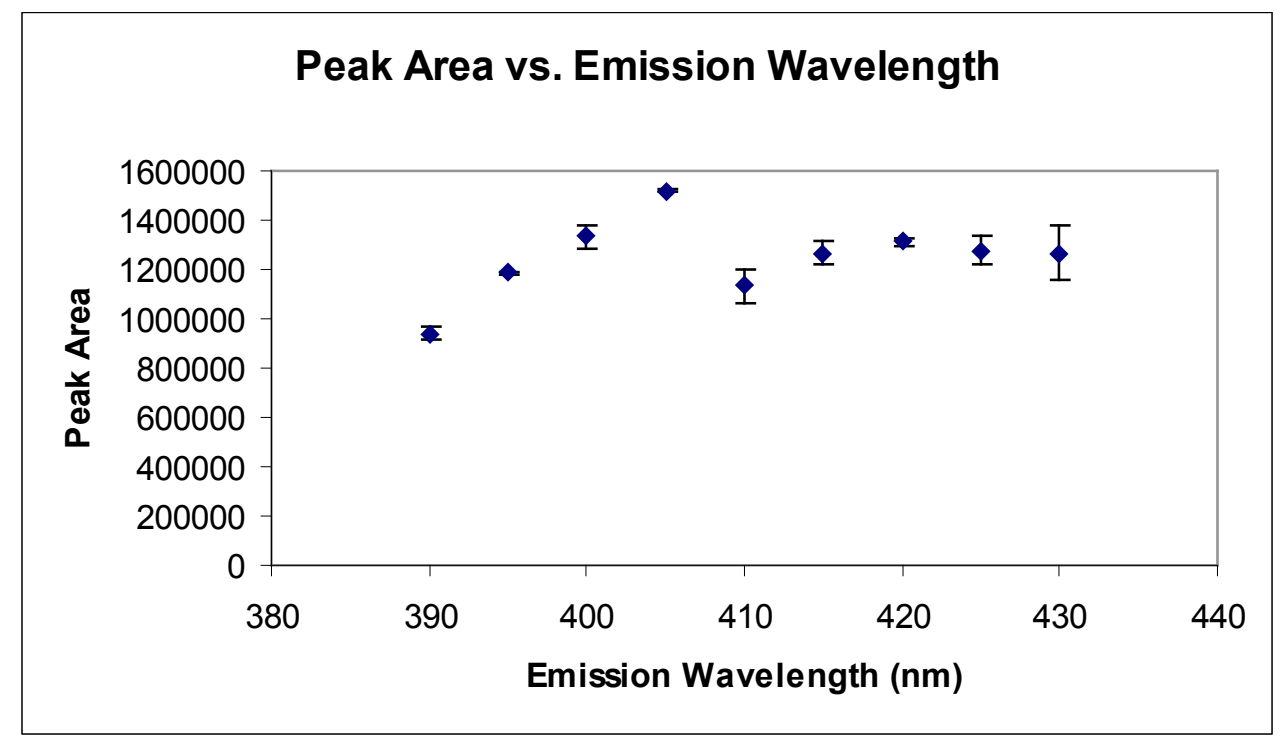

Figure 6.9. Effect of changing excitation wavelength on detection of hydrogen peroxide.

$\lambda_{\text {emission }}=405 \mathrm{~nm}$; an absorbance maximum for the POPHA dimer is present at $320 \mathrm{~nm}$.

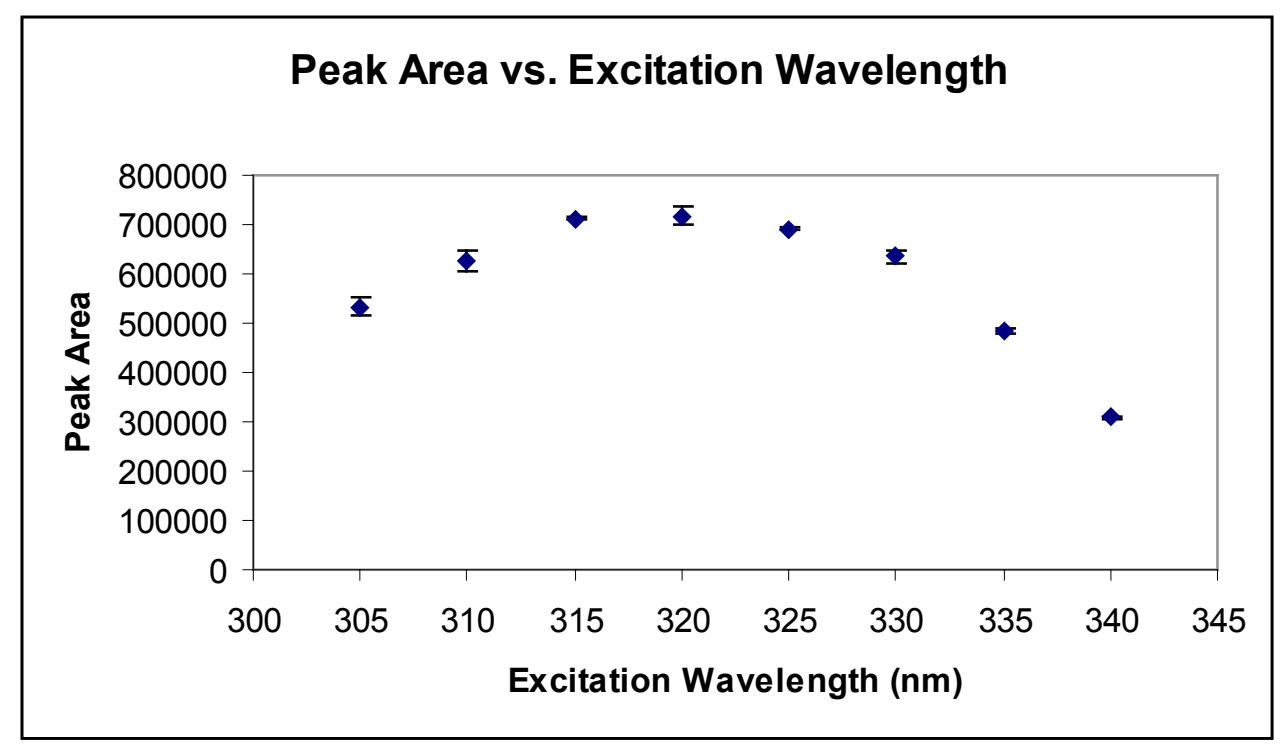


Table 6.10. Evaluation of additional excitation/emission wavelength pairs to optimize detection of hydrogen peroxide. The greatest peak area for hydrogen peroxide occurs with $\lambda_{\text {excitation }}=320 \mathrm{~nm}$ and $\lambda_{\text {emission }}=405 \mathrm{~nm}$

\begin{tabular}{|c|c|c|c|}
\hline Excitation $\lambda(\mathrm{nm})$ & Emission $\lambda(\mathrm{nm})$ & Ave Peak Area & $\%$ RSD Peak Area \\
\hline 318 & 405 & $1.21 \times 10^{6}$ & 3.8 \\
\hline 318 & 407 & $1.07 \times 10^{6}$ & 0.9 \\
\hline 320 & 403 & $9.19 \times 10^{5}$ & 2.3 \\
\hline 320 & 405 & $1.24 \times 10^{6}$ & 1.7 \\
\hline 320 & 407 & $9.42 \times 10^{5}$ & 2.0 \\
\hline 322 & 405 & $1.07 \times 10^{6}$ & 2.0 \\
\hline
\end{tabular}

Figure 6.10. Analysis of a $0.5 \mathrm{ppm}$ hydrogen peroxide standard by HPLC/FD.

Conditions: Dionex C-18 analytical column, isocratic elution with $1.00 \mathrm{mM} \mathrm{H}_{2} \mathrm{SO}_{4}$ and $0.10 \mathrm{mM}$ EDTA at flow rate $0.60 \mathrm{~mL} / \mathrm{min}, 20 \mu \mathrm{L}$ injection, reagent $8 \mu \mathrm{M}$ hemin $/ 80 \mu \mathrm{M}$ POPHA in ammonia buffer $\mathrm{pH} 9.5$ at flow rate $0.20 \mathrm{~mL} / \mathrm{min}$, post-column reactor $2.0 \mathrm{~m}$, $\mathrm{T}=25^{\circ} \mathrm{C}$, base $100 \mathrm{mM} \mathrm{NaOH}$ at flow rate $0.20 \mathrm{~mL} / \mathrm{min}, \ddot{\mathrm{e}}_{\mathrm{ex}}=320 \mathrm{~nm} \lambda_{\mathrm{em}}=405 \mathrm{~nm}$. Peak 1 , hydrogen peroxide Rt $5.152 \mathrm{~min}$.

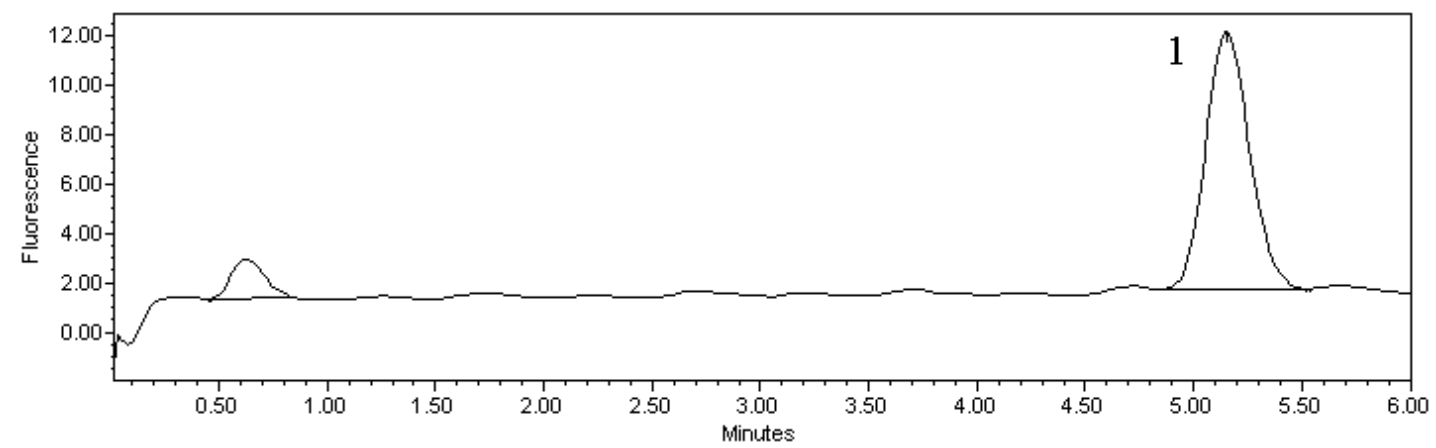


The analysis of a $0.5 \mathrm{ppm}$ hydrogen peroxide standard using the optimized HPLC/FD method is presented in Figure 6.10. The small peak at approximately 0.65 minutes is a system peak which was present in every sample, including water and mobile phase blank samples.

\section{Confirmation of hydrogen peroxide}

It was necessary to confirm the identity of the peak which was believed to represent hydrogen peroxide. In order to do this, the hemin/POPHA reagent solution was replaced with a water solution so that the hydrogen peroxide would not be destroyed. The base solution was also replaced with water. Three replicate $10 \mu \mathrm{L}$ injections of a standard solution of $300 \mu \mathrm{g} / \mathrm{mL}$ hydrogen peroxide were performed, for a total amount of $3 \mu \mathrm{g}$ hydrogen peroxide. Beginning at the time of sample injection, eluent fractions were collected in one minute intervals until seven minutes after injection. Liquid from each fraction was pipetted onto the surface of a commercial hydrogen peroxide test strip. The test was based on the colorimetric reaction of peroxide with 2,2'-azino-bis(3ethylbenzothiazoline)-6-sulfonate; the presence of a blue color on the test strip was indicative of the presence of peroxide. This test is not specific to hydrogen peroxide as both organic and inorganic peroxides in aqueous solution and organic solvents can be measured. According to the manufacturer, results were semi-quantitative over the range of $0.50 \mu \mathrm{g} / \mathrm{mL}-25.0 \mu \mathrm{g} / \mathrm{mL}$. For all three trials, only the fractions which contained hydrogen peroxide yielded a positive result on the test strips. Each of the positive fractions corresponded to the time interval of five minutes to six minutes post-injection when hydrogen peroxide was thought to elute. Given that $3 \mu \mathrm{g}$ of hydrogen peroxide 
were injected, and that each fraction collected contained approximately $1 \mathrm{~mL}$ of eluent $(1.00 \mathrm{~mL} / \mathrm{min} * 1 \mathrm{~min}=1 \mathrm{~mL})$, then the peroxide-containing fractions should have contained approximately $3 \mu \mathrm{g} / \mathrm{mL}$ hydrogen peroxide. However, the color on the test strips corresponded to the $0.5 \mu \mathrm{g} / \mathrm{mL}$ color on the test kit as before, indicating that the results from this kit are indeed only semi-quantitative. Nonetheless, these results confirm that the peak which elutes at 5.15 minutes is hydrogen peroxide. These results are in agreement with those obtained from the electrochemical system.

\section{Summary}

After evaluating three analytical columns and three mobile phase compositions, a method for the separation of hydrogen peroxide was optimized. The separation parameters involved a C-18 column with a $1.00 \mathrm{mM} \mathrm{H}_{2} \mathrm{SO}_{4} / 0.10 \mathrm{mM}$ EDTA mobile phase at a flow rate of $0.60 \mathrm{~mL} / \mathrm{min}$. Detection of hydrogen peroxide was based on the production of a fluorescent dimer from the reaction of hydrogen peroxide with $p$ hydroxyphenylacetic acid (POPHA) in the presence of a hemin catalyst. The composition

of the base solution, the flow rates for the base and reagent solution, the length, internal diameter, and temperature of the reaction tubing, and the excitation and emission wavelengths for fluorescent detection of the POPHA dimer were optimized for detection of hydrogen peroxide.

\section{Validation of HPLC/ED and HPLC/FD Methods}

Validation of the analytical methods which were developed for the analysis of hydrogen peroxide was required to prove that the methods are acceptable for forensic use. 
Validation included providing a demonstration of linearity, documentation of the limit of detection, demonstration of specificity and selectivity, demonstration of accuracy and precision, and demonstration of method robustness. A calibration curve was generated on the HPLC/FD system for hydrogen peroxide across the range of $0.5 \mathrm{ppm}-10.0 \mathrm{ppm}$. These data are presented in Figure 6.11. Peroxide concentrations greater than $10.0 \mathrm{ppm}$ resulted in detector saturation.

Figure 6.11. Calibration curve for hydrogen peroxide on the HPLC/FD system at standard conditions: $0.5 \mathrm{ppm}-10.0 \mathrm{ppm}$. Conditions as in Figure 6.10. Each data point represents the average peak area of three replicate injections. The curve is linear across this range.

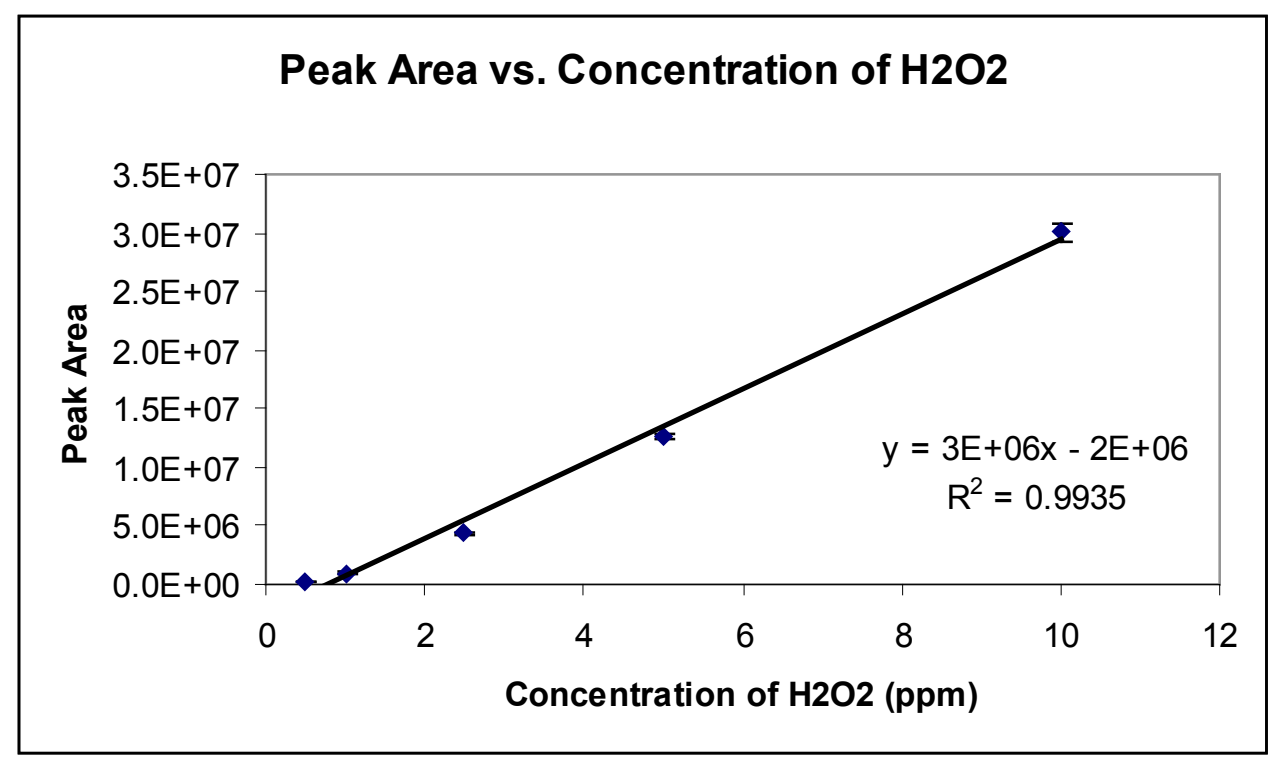

A calibration curve was generated on the HPLC/ED system in DC mode for hydrogen peroxide across the range of $0.25 \mathrm{ppm}-500 \mathrm{ppm}$. These data are presented in 
Figure 6.12. At sample concentrations greater than $500 \mathrm{ppm}$, the calibration became nonlinear and carryover was a problem. It is apparent from viewing the two calibration curves that the fluorescence system has a much more limited dynamic range than does the electrochemical system. In practice, peroxide samples are diluted such that the same sample at the same dilution can be analyzed on both systems simultaneously.

Figure 6.12. Calibration curve for hydrogen peroxide on the HPLC/ED system in DC mode at standard conditions: $0.25 \mathrm{ppm}-500 \mathrm{ppm}$. Conditions as in Figure 6.6. Each data point represents the average peak area of three replicate injections. The curve is linear across this range.

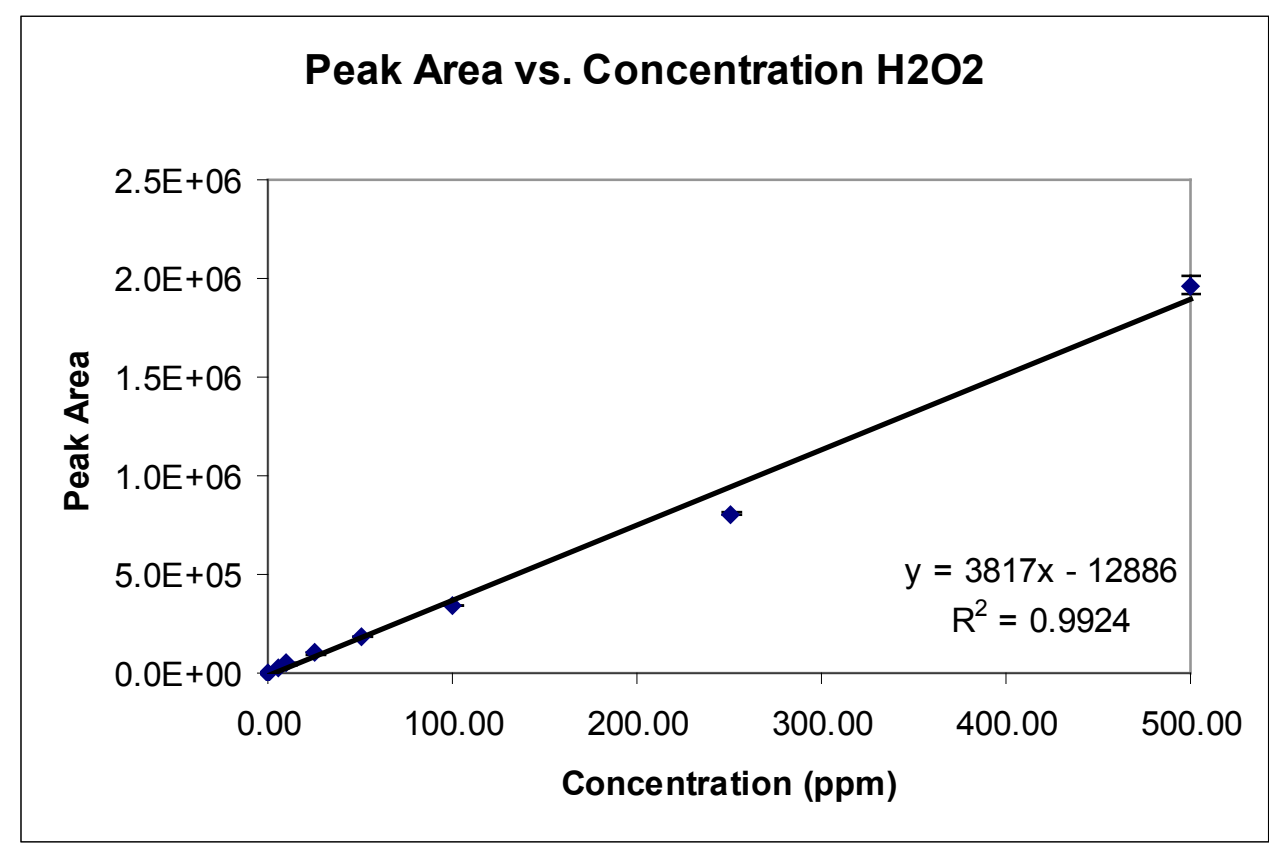

The limit of detection (LOD) is defined as the concentration which gives an instrument signal that is significantly different from the blank or background signal. In 
practical terms, this can be defined as the analyte concentration giving a signal equal to the blank signal ( $\mathrm{y}_{\mathrm{B}}$ ) plus three standard deviations of the blank, $\mathrm{s}_{\mathrm{B}}$ (see below) (116).

$$
\mathrm{y}-\mathrm{y}_{\mathrm{B}}=3 \mathrm{~s}_{\mathrm{B}}
$$

To calculate the limit of detection using this format, ten replicate injections were performed of a hydrogen peroxide standard whose concentration was very near to the theorized instrument detection limit. The average and standard deviation of these measurements were determined, and this information was substituted into the above equation. The resulting y-value was then converted to a concentration value. This value corresponded to the limit of detection in terms of sample concentration. The LOD for hydrogen peroxide on the HPLC/ED system in DC mode was $0.02 \mu \mathrm{g} / \mathrm{mL}$, while the LOD for hydrogen peroxide on the HPLC/FD system was $0.19 \mu \mathrm{g} / \mathrm{mL}$. It should be noted that the limit of detection for hydrogen peroxide on the electrochemical method is an order of magnitude lower than the limit of detection for hydrogen peroxide on the fluorescence method.

It was important to evaluate the robustness of the HPLC/ED and HPLC/FD methods because they were designed with the intention that they would be applied to the analysis of post-blast samples. Therefore, a brief series of experiments were conducted to evaluate the effects of several different types of compounds that might reasonably be expected to interfere with the detection of hydrogen peroxide. In this study, three groups of compounds were tested: the inorganic anions nitrate, nitrite, chloride, chlorate, and perchlorate; the organic acids ascorbic acid, citric acid, formic acid, lactic acid, and oxalic acid; and the sugars glucose, fructose, and sucrose. Each analyte was tested individually at a concentration of $100 \mu \mathrm{M}$ on both the HPLC/FD system, and the 
HPLC/ED system in DC mode. The results from the analysis of mixtures of inorganic anions with a hydrogen peroxide standard are presented in Table 6.11.

Table 6.11. Effect of the presence of $100 \mu \mathrm{M}$ of an inorganic anion on the peak area of $100 \mu \mathrm{M} \mathrm{H}_{2} \mathrm{O}_{2}$. Conditions as in Figures 6.6 and 6.10. The presence of the inorganic anions resulted in minimal suppression of or addition to the hydrogen peroxide signal. No additional peaks were noted on either system due to the presence of the inorganic anions.

\begin{tabular}{|c|c|c|c|c|}
\hline $\begin{array}{c}\text { Sample } \\
(100 \mu M)\end{array}$ & $\begin{array}{c}\text { Peak Area } \\
\text { HPLC/ED }\end{array}$ & $\begin{array}{c}\text { (Peak Area HP) } \\
/ \text { (Peak Area } \\
\text { HP }+ \text { Anion) }\end{array}$ & $\begin{array}{c}\text { Peak Area } \\
\text { HPLC/FD }\end{array}$ & $\begin{array}{c}\text { (Peak Area HP) } \\
/ \text { (Peak Area } \\
\text { HP }+ \text { Anion) }\end{array}$ \\
\hline $\mathrm{H}_{2} \mathrm{O}_{2}$ & $2.40 \times 10^{4}$ & 1.00 & $1.23 \times 10^{7}$ & 1.00 \\
\hline $\mathrm{H}_{2} \mathrm{O}_{2}+\mathrm{NO}_{2}^{-}$ & $2.30 \times 10^{4}$ & 1.04 & $1.19 \times 10^{7}$ & 1.09 \\
\hline $\mathrm{H}_{2} \mathrm{O}_{2}+\mathrm{ClO}_{3}^{-}$ & $2.30 \times 10^{4}$ & 1.04 & $1.21 \times 10^{7}$ & 1.02 \\
\hline $\mathrm{H}_{2} \mathrm{O}_{2}+\mathrm{Cl}^{-}$ & $2.30 \times 10^{4}$ & 1.04 & $1.21 \times 10^{7}$ & 1.01 \\
\hline $\mathrm{H}_{2} \mathrm{O}_{2}+\mathrm{NO}_{3}^{-}$ & $2.30 \times 10^{4}$ & 1.04 & $1.20 \times 10^{7}$ & 1.03 \\
\hline $\mathrm{H}_{2} \mathrm{O}_{2}+\mathrm{ClO}_{4}^{-}$ & $2.40 \times 10^{4}$ & 1.00 & $1.22 \times 10^{7}$ & 1.01 \\
\hline
\end{tabular}

Of the potential interferences which were tested, not one yielded a detectable signal on the fluorescence system, and only ascorbic acid gave a signal on the electrochemical system. This signal took the form of a broad peak which eluted at approximately 2.12 minutes. This peak was absent when ascorbic acid was injected at a concentration of $60 \mu \mathrm{M}$. Samples which consisted of $100 \mu \mathrm{M}$ hydrogen peroxide with $100 \mu \mathrm{M}$ of each of the potential interferences were also analyzed on both systems. The 
presence of the additional analytes with the hydrogen peroxide standard resulted in minimal suppression of or addition to the hydrogen peroxide signal.

Studies were conducted to determine the within-day reproducibility of the retention time and peak area of standard samples of hydrogen peroxide on both the HPLC/ED and HPLC/FD systems. This test involved the analysis of ten replicate injections of both a $0.5 \mathrm{ppm}$ hydrogen peroxide standard, and a $5.0 \mathrm{ppm}$ hydrogen peroxide standard. These data are presented in Table 6.12.

Table 6.12. Reproducibility data for the retention time and peak area of $0.5 \mathrm{ppm}$ and 5.0 ppm hydrogen peroxide. For both methods, the between-day variability of the peak area and retention time of hydrogen peroxide was greater than the within-day variability.

\begin{tabular}{|c|c|c|c|c|c|c|}
\hline Method & & Sample & $\begin{array}{l}\text { Ave Rt } \\
\text { (min) }\end{array}$ & $\begin{array}{c}\% R S D \\
R t\end{array}$ & $\begin{array}{c}\text { Ave Peak } \\
\text { Area }\end{array}$ & $\begin{array}{c}\% R S D \\
\text { Area }\end{array}$ \\
\hline \multirow{4}{*}{ HPLC-FD } & \multirow{2}{*}{ Within-Day } & $0.5 \mathrm{ppm}$ & 5.150 & 0.11 & $219 \times 10^{3}$ & 6.1 \\
\hline & & $5.0 \mathrm{ppm}$ & 5.166 & 0.01 & $1.22 \times 10^{6}$ & 1.0 \\
\hline & \multirow{2}{*}{ Between Day } & $0.5 \mathrm{ppm}$ & 5.160 & 0.15 & $207 \times 10^{3}$ & 18 \\
\hline & & $5.0 \mathrm{ppm}$ & 5.165 & 0.01 & $1.21 \times 10^{6}$ & 4.6 \\
\hline \multirow{4}{*}{ HPLC-ED } & \multirow{2}{*}{ Within-Day } & $0.5 \mathrm{ppm}$ & 1.910 & 0.18 & $5.00 \times 10^{3}$ & 12 \\
\hline & & $5.0 \mathrm{ppm}$ & 1.875 & 0.01 & $39.5 \times 10^{3}$ & 1.3 \\
\hline & \multirow{2}{*}{ Between Day } & $0.5 \mathrm{ppm}$ & 1.890 & 0.25 & $6.00 \times 10^{3}$ & 17 \\
\hline & & $5.0 \mathrm{ppm}$ & 1.875 & 0.03 & $39.5 \times 10^{3}$ & 1.8 \\
\hline
\end{tabular}


To explore the between-day reproducibility of each HPLC method, peak area and retention time data for standard solutions of hydrogen peroxide at $0.5 \mathrm{ppm}$ and $5.0 \mathrm{ppm}$ were compared over ten days of data analysis. These data are also presented in Table 6.12. The variability of the retention time for both standards on both methods was less than or equal to $0.25 \%$, which is typical for HPLC. For both methods, the between-day variability of the peak area and retention time of hydrogen peroxide was greater than the within-day variability.

\section{E. Conclusions}

Two methods were developed for the analysis of trace levels of hydrogen peroxide: HPLC/FD and HPLC/ED in DC mode. Each method offers the benefits of a low limit of detection $(0.02 \mu \mathrm{g} / \mathrm{mL}$ for the HPLC/ED system, and $0.19 \mu \mathrm{g} / \mathrm{mL}$ for the HPLC/FD system), a linear dynamic range from $0.5 \mathrm{ppm}-10.0 \mathrm{ppm}$, selectivity to hydrogen peroxide, and insensitivity to a select group of potential interferences. The reproducibility of the retention time of hydrogen peroxide for both methods is excellent, Concurrent use of the HPLC/ED and HPLC/FD methods permits unequivocal detection of hydrogen peroxide. These techniques will be applied to the analysis of a variety of post-blast samples in the following chapter. 


\section{Chapter VII. Analysis of Field Samples by HPLC/ED and HPLC/FD}

\section{A. Introduction}

Following optimization of the electrochemical and fluorescence methods for the separation and detection of hydrogen peroxide, experiments were conducted to explore the effects of residue collection, storage, and extraction which would permit maximum recovery of hydrogen peroxide while minimizing the inclusion of interfering compounds. Three different extraction protocols were reviewed. In addition, two types of packaging materials and three different storage conditions were evaluated to determine the combination which best preserved hydrogen peroxide residues for later analysis.

A survey was conducted to determine at what levels hydrogen peroxide was present on surfaces in an enclosed, indoor environment. While the presence of hydrogen peroxide in the air and water of outdoor settings has been well documented (141) (143) (159), the presence of hydrogen peroxide in indoor settings has not been as thoroughly surveyed. This is an important issue to explore as hydrogen peroxide may be present from certain cleaning agents which are utilized in indoor settings. Residues from hydrogen peroxide-based cleaning agents could confuse data interpretation if present on surfaces which are sampled for post-blast residue collection. A similar problem can arise if products treated with hydrogen peroxide are utilized in the process of collecting samples.

It was important to apply the optimized electrochemical and fluorescence methods to the analysis of as many different types of samples as possible. While the analysis of intact and post-blast concentrated hydrogen peroxide/fuel mixtures were of primary 
interest in this work, the analysis of organic peroxides and other peroxide-based explosives (both intact and post-blast) have also been investigated.

\section{B. Effect of Mode of Application, Storage, and Extraction on Recovery of $\mathrm{H}_{2} \mathrm{O}_{2}$}

A method for applying a reproducible amount of hydrogen peroxide to a series of porous and non-porous substrates was developed in order to test the effects of storage conditions on the recovery of hydrogen peroxide residues. For this test, the substrates included cotton swabs, aluminum metal plates, paint chips, smooth plastic chips, rough plastic chips, polymer-coated steel plates, and bare steel plates. An aliquot of $75.0 \mu \mathrm{L}$ of $30 \%$ hydrogen peroxide was applied to the surface of each substrate, for a total applied amount of $225 \mathrm{mg}$ hydrogen peroxide. The first series of substrates were allowed to air dry on the surface of a laboratory bench at approximately $23^{\circ} \mathrm{C}$, a process which took approximately sixteen hours. Once the substrates were dry, they were extracted in 1.00 $\mathrm{mL}$ of 18.3 M $\Omega \mathrm{DI}$ water and analyzed on the electrochemical and fluorescence systems. The second series of substrates were dried with a heat gun, a process which took approximately fifteen minutes. The substrates were immediately extracted in $1.00 \mathrm{~mL}$ of 18.3 M $\Omega$ DI water and analyzed on the electrochemical and fluorescence systems. The third series of substrates was dried with the heat gun, and then allowed to sit uncovered on the laboratory bench at approximately $23^{\circ} \mathrm{C}$ for twenty-four hours prior to analysis. After twenty-four hours passed, the substrates were extracted in $1.00 \mathrm{~mL}$ of $18.3 \mathrm{M} \Omega \mathrm{DI}$ water and analyzed on the electrochemical and fluorescence systems. Three point calibration curves were generated on the HPLC/ED system in PAD mode so that the amount of hydrogen peroxide present in each sample could be determined. The presence 
of hydrogen peroxide in each sample was confirmed by the HPLC/FD method. The results of the analyses by HPLC/ED in PAD mode are presented in Table 7.1.

Table 7.1. Mode of application versus recovery of hydrogen peroxide residue. Aliquots of $225 \mathrm{mg} \mathrm{H} \mathrm{H}_{2} \mathrm{O}_{2}$ were applied to the surface of seven substrates and allowed to dry. Peroxide residues were recovered from the substrates by a direct water rinse procedure. The type of drying procedure and amount of time the peroxide residues were exposed to ambient air affected the recovery of the hydrogen peroxide residues. Standard HPLC/ED in PAD mode analysis conditions were applied.

\begin{tabular}{|c|c|c|c|c|c|c|}
\hline $\begin{array}{c}\text { Sample } \\
(n=3)\end{array}$ & \multicolumn{2}{|c|}{$\begin{array}{c}\text { Air Dry } \\
(\sim 16 \text { hours })\end{array}$} & \multicolumn{2}{c|}{$\begin{array}{c}\text { Heat Gun } \\
(\sim 15 \text { minutes })\end{array}$} & \multicolumn{2}{c|}{$\begin{array}{c}\text { Heat Gun plus } 24 \\
\text { hours of exposure }\end{array}$} \\
\hline & $\begin{array}{c}\mu \mathrm{g} \mathrm{HP} \\
\text { recovered }\end{array}$ & $\begin{array}{c}\% \mathrm{RSD} \\
\mathrm{HP}\end{array}$ & $\begin{array}{c}\mu \mathrm{g} \mathrm{HP} \\
\text { recovered }\end{array}$ & $\begin{array}{c}\% \mathrm{RSD} \\
\mathrm{HP}\end{array}$ & $\begin{array}{c}\mu \mathrm{g} \mathrm{HP} \\
\text { recovered }\end{array}$ & $\begin{array}{c}\% \mathrm{RSD} \\
\mathrm{HP}\end{array}$ \\
\hline Cotton Swab & 810 & 0.7 & 15,500 & 0.29 & 7,630 & $2.6 \%$ \\
\hline Paint Chip & 0.34 & 4.2 & 19.13 & 0.24 & not detected & $\mathrm{n} / \mathrm{a}$ \\
\hline Smooth Plastic & 0.06 & 14 & 3.30 & 0.96 & not detected & $\mathrm{n} / \mathrm{a}$ \\
\hline Rough Plastic & 0.06 & 22 & 9.32 & 0.33 & not detected & $\mathrm{n} / \mathrm{a}$ \\
\hline Aluminum Plate & 0.05 & 11 & 2.44 & 0.07 & not detected & $\mathrm{n} / \mathrm{a}$ \\
\hline Uncoated Metal & 0.06 & 16 & 2.41 & 0.73 & not detected & $\mathrm{n} / \mathrm{a}$ \\
\hline Coated Metal & 0.23 & 1.9 & 1.46 & 1.25 & 0.05 & 65 \\
\hline
\end{tabular}


Examination of the data presented in Table 7.1 reveals that use of the heat gun to dry the samples resulted in an amount of recovered peroxide which was up to two orders of magnitude greater than for samples which were allowed to air dry. The RSD values for the heat-dried samples were also much lower than for the air-dried samples. It is theorized that use of the heat gun resulted in flash-drying of the aqueous hydrogen peroxide, a process which fixed more of the peroxide residues on the substrate surface than did air-drying. Even though $225 \mathrm{mg}$ of peroxide was applied to each substrate, less than $1 \mu \mathrm{g}$ was recovered from six of the seven air-dried substrates. It should be noted that recovery of peroxide from the air-dried cotton swab was more than 1000 times greater than for the air-dried non-porous substrates. Even though more peroxide was recovered using the heat gun to dry the samples, the amount of peroxide recovered from the heatdried samples was still small. Fewer than $50 \mu \mathrm{g}$ of peroxide was recovered from six of the seven heat-dried substrates. Again, recovery of peroxide from the heat-dried samples was greatest from the cotton swab; nearly 1000 times greater than for the non-porous materials. While the use of the heat gun allowed less peroxide to evaporate than did airdrying, the volatile nature of hydrogen peroxide clearly made recovery of trace levels of the material difficult. Even when the heat gun was used to dry the substrates, leaving the substrates exposed to ambient conditions for twenty-four hours allowed further evaporation of hydrogen peroxide. Of the seven substrates which were treated this way, hydrogen peroxide was only detected on two of the materials: the cotton swab and the plastic coated metal. For both of these materials, the amount of peroxide which was recovered was less than what was recovered after heat drying followed by immediate analysis. The level of peroxide present on the other five substrates was below the limit of 
detection for both the fluorescence and electrochemical methods. It should be noted that the RSD values for the amount of peroxide recovered from the substrates were the greatest for samples with the least amount of peroxide in them. This is to be expected as the variability in the measurement increases as the limit of detection is approached (see Chapter 6).

Experiments were performed to determine how different extraction protocols would affect the recovery of hydrogen peroxide from the surface of a given substrate. For this test, aliquots of $75 \mu \mathrm{L}$ of $5 \%$ hydrogen peroxide (equivalent to $4.1 \mathrm{mg} \mathrm{H}_{2} \mathrm{O}_{2}$ ) were applied to the surface of three paint chips, and the heat gun was used to dry them. The hydrogen peroxide residues were extracted from the surface of the chips by one of three protocols: direct washing with $1.00 \mathrm{~mL}$ of $18.3 \mathrm{M} \Omega \mathrm{DI}$ water, swabbing with a dry cotton swab, or swabbing with a cotton swab moistened with $0.50 \mathrm{~mL}$ of $18.3 \mathrm{M} \Omega$ DI water. To keep the volume of water applied to each swab equal, the dry swabs were extracted in 3 $\mathrm{mL}$ of $18.3 \mathrm{M} \Omega \mathrm{DI}$ water while the wet swabs were extracted in $2.50 \mathrm{~mL}$ of $18.3 \mathrm{M} \Omega \mathrm{DI}$ water. The difference in extraction volumes between the swabs and the direct rinsing procedures was included in the calculation of the total mass of recovered hydrogen peroxide. Ten replicates were performed of each extraction protocol. The extracts were analyzed by HPLC/ED in DC mode. Three point calibration curves were generated on the HPLC/ED system in DC mode so that the amount of hydrogen peroxide present in each sample could be determined. The presence of hydrogen peroxide was confirmed by HPLC/FD analysis. It was theorized the direct rinse procedure would permit the greatest recovery of hydrogen peroxide because the mechanical action of rinsing the substrates 
with the water should wash the peroxide residues from the surface of the substrates. The results of this experiment are presented in Table 7.2.

Table 7.2. Effect of mode of extraction on recovery of hydrogen peroxide residue. Aliquots of $4.1 \mathrm{mg} \mathrm{H}_{2} \mathrm{O}_{2}$ were applied to the surface of paint chips and dried with the heat gun. Peroxide residues were recovered from the paint chips by a direct water rinse procedure, wet swabbing, or dry swabbing. Standard HPLC/ED in DC mode analysis conditions were applied. Ten replicates were performed of each extraction procedure.

\begin{tabular}{|c|c|c|c|}
\hline $\begin{array}{c}\text { Extraction } \\
\text { Procedure }\end{array}$ & $\begin{array}{c}\text { Average Mass of } \\
\text { Recovered } \mathrm{H}_{2} \mathrm{O}_{2}\end{array}$ & Standard Deviation & RSD of Recovered \\
\hline Dry Swab & $2.36 \mu \mathrm{g}$ & $\pm 2.7 \mu \mathrm{g}$ & $\mathrm{H}_{2} \mathrm{O}_{2}$ \\
\hline Wet Swab & $1.82 \mu \mathrm{g}$ & $\pm 1.6 \mu \mathrm{g}$ & $110 \%$ \\
\hline Direct Rinse & $7.89 \mu \mathrm{g}$ & $\pm 12 \mu \mathrm{g}$ & $150 \%$ \\
\hline
\end{tabular}

It is apparent from viewing Table 7.2 that of the $4.1 \mathrm{mg}$ of hydrogen peroxide which were applied to the surface of the test materials, on average approximately $0.1 \%$ was actually recovered by each of the three extraction protocols. It was theorized that the direct rinsing procedure would result in the greatest recovery of hydrogen peroxide. Statistical analysis was performed on the data set using Analysis of Variance (ANOVA) to determine whether the difference in the average recovery of hydrogen peroxide for the three extraction protocols was significant at 95\% confidence interval. The null hypothesis was that there was no significant difference. If this hypothesis was correct, then the between-sample estimate of the variance should not differ significantly from the within- 
sample estimate of the variance. A one-tailed $F$ test was used to test the hypothesis.

Because the calculated value of $F$ was less than the critical value of $F$ with $\mathrm{P}=0.05$, the null hypothesis stood, meaning that there was no significant difference in the recovery of peroxide residue for the three extraction protocols. This result is not surprising, given the very high standard deviation that each procedure carried.

Table 7.3. Recovery of hydrogen peroxide residue from four different substrates.

Aliquots of $4.1 \mathrm{mg}$ hydrogen peroxide were applied to swatches of denim fabric, blocks of concrete, paint chips, and steel plates, and dried with the heat gun. The materials were stored under varying conditions of storage temperature, packaging material, and duration of storage. Peroxide residues were recovered using a dry swabbing technique. Standard HPLC/ED in DC mode analysis conditions were applied.

\begin{tabular}{|c|c|c|c|c|c|c|c|}
\hline Sample & Temp & Day 1 & Day 1 & Day 3 & Day 3 & Day 7 & Day 7 \\
& & Nylon & Paint Can & Nylon & Paint Can & Nylon & Paint Can \\
\hline \multirow{3}{*}{ Denim } & $23^{\circ} \mathrm{C}$ & Yes & Yes & Yes & Yes & No & No \\
\cline { 2 - 8 } & $-15^{\circ} \mathrm{C}$ & Yes & Yes & Yes & Yes & Yes & Yes \\
\hline \multirow{3}{*}{ Concrete } & $23^{\circ} \mathrm{C}$ & Yes & Yes & Yes & Yes & Yes & Yes \\
\cline { 2 - 8 } & $-15^{\circ} \mathrm{C}$ & Yes & Yes & Yes & Yes & Yes & Yes \\
\hline \multirow{2}{*}{ Paint } & $23^{\circ} \mathrm{C}$ & Yes & Yes & Yes & Yes & No & No \\
\cline { 2 - 8 } Chip & $-15^{\circ} \mathrm{C}$ & Yes & Yes & Yes & Yes & Yes & Yes \\
\hline \multirow{2}{*}{ Steel } & $23^{\circ} \mathrm{C}$ & Yes & Yes & Yes & Yes & No & No \\
\cline { 2 - 8 } Plate & $-15^{\circ} \mathrm{C}$ & Yes & Yes & Yes & Yes & Yes & Yes \\
\hline
\end{tabular}


A series of experiments were performed to explore the stability of hydrogen peroxide on a variety of porous and non-porous substrates under varying conditions for type of packaging material, storage temperature, and duration of storage. The substrates included denim fabric, concrete blocks, paint chips, and steel plates. The peroxide-treated substrates were stored either in paint cans or in a single layer of heat-sealed nylon bags. The packaged test materials were stored under one of two temperature/humidity conditions: room temperature $\left(T=23^{\circ} \mathrm{C}\right)$, and freezer $\left(\mathrm{T}=-15^{\circ} \mathrm{C}\right)$. The materials were analyzed after one day, three days, and seven days of storage. The HPLC/ED analysis was performed as a qualitative analysis only. Samples were reported as positive if the signal strength of hydrogen peroxide was greater than the limit of detection $0.02 \mu \mathrm{g} / \mathrm{mL}$ for the HPLC/ED system. The results of this study are presented in Table 7.3.

Examination of this data set reveals that of the three variables (storage temperature, packaging material, and duration of storage), storage temperature had the strongest influence on whether or not hydrogen peroxide residues were recovered. Hydrogen peroxide residues were detected on all four substrates during all seven days of storage only when the materials were kept at $\mathrm{T}=-15^{\circ} \mathrm{C}$. When the materials were maintained at $\mathrm{T}=23^{\circ} \mathrm{C}$, by the seventh day of storage hydrogen peroxide residues were detected on just one substrate (the concrete blocks). Hydrogen peroxide residues were detected on the denim fabric, paint chip, and steel plate only during the first three days of storage. Cold storage may have prevented hydrogen peroxide from evaporating, degrading, or a combination of both events. The lack of humidity in the freezer may also have helped to preserve the hydrogen peroxide residues. It should be noted that the peroxide-treated substrates were maintained in dark areas during the course of the 
experiment so that exposure to UV light did not contribute to the degradation of the hydrogen peroxide residues. These results indicate that substrates which contain peroxide should be stored in cold temperatures in order to recover as much hydrogen peroxide residue as possible. The type of packaging material used did not appear to result in a difference in the detection of hydrogen peroxide. At this time, no advantage can be seen in using one type of packaging material over the other.

\section{Evaluation of Cotton Sampling Media}

While a variety of different materials can be utilized for the sampling of explosives and their residues, including adhesive-coated aluminum stubs (37), solid phase micro-extraction fibers (160), and hand-held vacuum devices (161), cotton swabs are a particularly attractive sampling media given their low cost, ease of use, and general availability. Cotton swabs have been demonstrated to collect both organic and inorganic compounds (38); they can be used either dry or moistened with solvent. Also, transportation and storage of these materials is relatively simple.

In a forensic setting, certain explosives analysis protocols require the use of cotton swabs which have been previously cleaned and tested, and are known to be free of interfering components. Other protocols are less stringent, and may not place any requirements on the pre-treatment of explosives sampling materials. However, blanks, controls, and standards must always be run as part of a properly validated procedure. While the average cotton swab purchased at a local drug store may be free of RDX or TNT, this may not be the case for hydrogen peroxide, particularly given the industrial applications of the compound as both a whitening and anti-microbial agent. Therefore, 
prior to the use of any cotton swab for residue collection, blank cotton swabs were tested for the presence of interfering components.

A total of seven different types of blank white cotton swabs were evaluated to determine their suitability for the collection of hydrogen peroxide residues, including cotton balls, cotton rounds, and cotton squares. Five swabs were tested for each brand and type of cotton. It was particularly important to determine whether or not the cotton would yield any peaks which might interfere with the detection of hydrogen peroxide. The packaging material for two of the seven types of cotton swabs utilized in this study (CVS Regular Cotton Balls and Organic Essentials Cotton Balls) stated that the cotton had been whitened with hydrogen peroxide. It was hypothesized that hydrogen peroxide residues would be present in extracts from these brands of cotton. Because the packaging material for the other five types of cotton did not state what type of chemical had been employed during the whitening process, it was also possible that hydrogen peroxide residues might be detected in extracts from these brands of cotton.

Extracts from each sample of cotton were analyzed by HPLC/FD and HPLC/ED in DC mode. Three point calibration curves were generated on the HPLC/ED system in DC mode so that the amount of hydrogen peroxide present in each sample of cotton could be determined. The HPLC/FD analyses were performed as qualitative tests only. The results of the analysis of all seven different types of cotton are presented in Table 7.4. The average amount of hydrogen peroxide present in each type of cotton swab was calculated. 
Table 7.4. Results of analysis of seven types of blank cotton swabs. Peroxide residues were extracted from the swabs with water. Standard HPLC/FD and HPLC/ED in DC mode analysis conditions were applied. The amount of hydrogen peroxide present in each type of cotton was determined by HPLC/ED in DC mode.

\begin{tabular}{|c|c|c|c|c|}
\hline $\begin{array}{c}\text { Type of cotton swab } \\
(n=5)\end{array}$ & $\begin{array}{c}\text { Ave amount of } \\
\mathrm{H}_{2} \mathrm{O}_{2} \mathrm{per}\end{array}$ & Stnd & Ave Mass & $\mu \mathrm{g} \mathrm{H}_{2} \mathrm{O}_{2}$ \\
& Cotton) & per gram \\
& $(n=5)$ & $\mathrm{H}_{2} \mathrm{O}_{2}$ & $(n=5)$ & of cotton \\
\hline CVS Cotton Squares & $1.39 \mu \mathrm{g}$ & $\pm 0.07 \mu \mathrm{g}$ & $0.505 \mathrm{~g}$ & 2.75 \\
\hline CVS Regular Cotton Balls & $0.21 \mu \mathrm{g}$ & $\pm 0.02 \mu \mathrm{g}$ & $0.213 \mathrm{~g}$ & 0.99 \\
\hline CVS Cotton Rounds & $0.34 \mu \mathrm{g}$ & $\pm 0.05 \mu \mathrm{g}$ & $0.576 \mathrm{~g}$ & 0.59 \\
\hline Organic Essentials Cotton Balls & $0.37 \mu \mathrm{g}$ & $\pm 0.02 \mu \mathrm{g}$ & $0.674 \mathrm{~g}$ & 0.55 \\
\hline Johnson \& Johnson Cotton Balls & $0.18 \mu \mathrm{g}$ & $\pm 0.01 \mu \mathrm{g}$ & $0.325 \mathrm{~g}$ & 0.55 \\
\hline CVS Organic Cotton Balls & $0.30 \mu \mathrm{g}$ & $\pm 0.01 \mu \mathrm{g}$ & $0.632 \mathrm{~g}$ & 0.47 \\
\hline Wal-Mart Cotton Balls & $0.11 \mu \mathrm{g}$ & $\pm 0.04 \mu \mathrm{g}$ & $0.632 \mathrm{~g}$ & 0.16 \\
\hline
\end{tabular}

In viewing Table 7.4, it is apparent that hydrogen peroxide was detected in all but one of the seven brands of cotton which were tested. Wal-Mart brand cotton balls had the lowest average amount of hydrogen peroxide per swab at $0.11 \mu \mathrm{g}$, while CVS cotton squares had the highest average amount of hydrogen peroxide at $1.39 \mu \mathrm{g}$. The average mass of the cotton swabs varied widely amongst the different types of cotton. Regular cotton balls from CVS had the lowest average mass at $0.213 \mathrm{~g}$, while Organic Essentials cotton balls had the highest average mass at $0.674 \mathrm{~g}$. In order to normalize the data so 
that a direct comparison could be made of hydrogen peroxide content amongst the different types of cotton, the following calculation was performed:

Average amount of $\mathrm{H}_{2} \mathrm{O}_{2} /$ Average mass of cotton $=\mu \mathrm{g} \mathrm{H}_{2} \mathrm{O}_{2} / \mathrm{g}$ cotton

Wal-Mart cotton balls had the lowest value at $0.16 \mu \mathrm{g} \mathrm{H}_{2} \mathrm{O}_{2} / \mathrm{g}$ cotton, while CVS cotton squares had the highest value at $2.75 \mu \mathrm{g} \mathrm{H}_{2} \mathrm{O}_{2} / \mathrm{g}$ cotton. These data indicate that any of the types of cotton swabs would be a poor choice for the collection of trace levels of hydrogen peroxide without prior treatment to remove the hydrogen peroxide from the cotton. The results from the analysis of one of the Wal-Mart cotton balls are presented in Figures 7.1 and 7.2. These results are typical for all of the types of cotton which were analyzed in that no peaks other than hydrogen peroxide are present in the chromatograms.

Figure 7.1. Analysis of a Wal-Mart cotton ball by HPLC/ED in DC Mode. Conditions: Dionex CarboPac PA10 analytical column, isocratic elution with $150 \mathrm{mM} \mathrm{NaOAc}$ at $\mathrm{pH}$ 10.5, flow rate $1.00 \mathrm{~mL} / \mathrm{min}, 100 \mu \mathrm{L}$ injection, $\mathrm{T}=45^{\circ} \mathrm{C}, \mathrm{DC}$ Mode with $\mathrm{E}=+0.4 \mathrm{~V}$. Peak 1, hydrogen peroxide, Rt 1.926 min. Hydrogen peroxide is present in the "blank" cotton.

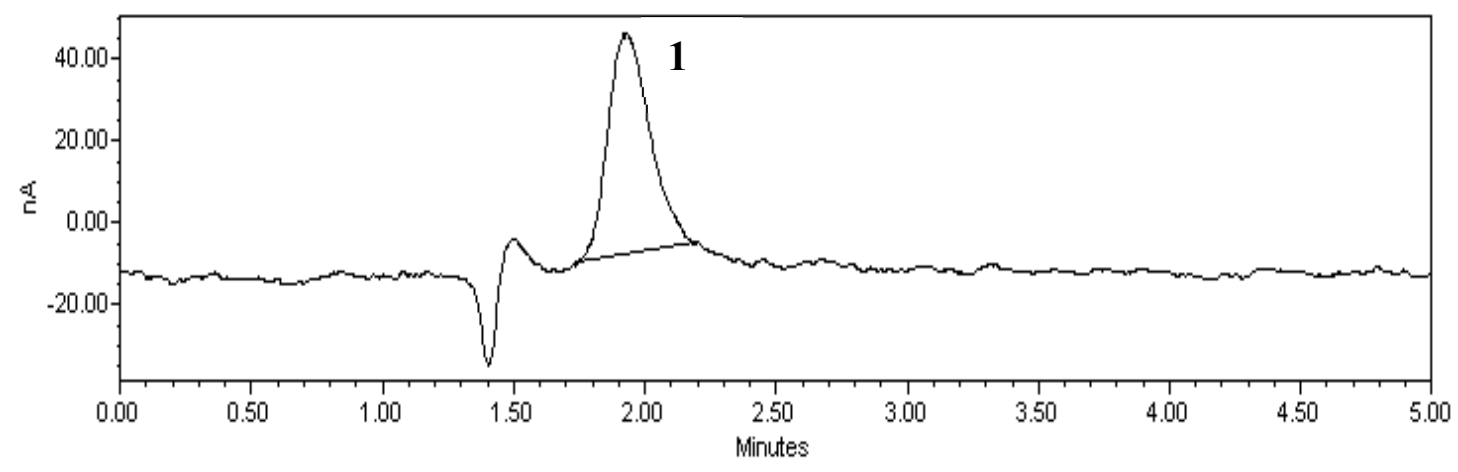


Figure 7.2. Analysis of a Wal-Mart cotton ball by HPLC/FD. Conditions: Dionex C-18 analytical column, isocratic elution with $1 \times 10^{-3} \mathrm{M} \mathrm{H}_{2} \mathrm{SO}_{4} / 1 \times 10^{-4} \mathrm{M}$ EDTA at flow rate $0.60 \mathrm{~mL} / \mathrm{min}, 100 \mu \mathrm{L}$ injection, reagent $8 \mu \mathrm{M}$ hemin $/ 80 \mu \mathrm{M}$ POPHA in ammonia buffer $\mathrm{pH} 9.5$ at flow rate $0.20 \mathrm{~mL} / \mathrm{min}$, post-column reactor $2 \mathrm{~m}, \mathrm{~T}=25^{\circ} \mathrm{C}$, base $0.1 \mathrm{M} \mathrm{NaOH}$ at flow rate $0.20 \mathrm{~mL} / \mathrm{min}, \lambda_{\mathrm{ex}}=320 \mathrm{~nm}, \lambda_{\mathrm{em}}=405 \mathrm{~nm}$. Peak 1 , hydrogen peroxide, Rt 5.237 min. Hydrogen peroxide is present in the "blank" cotton.

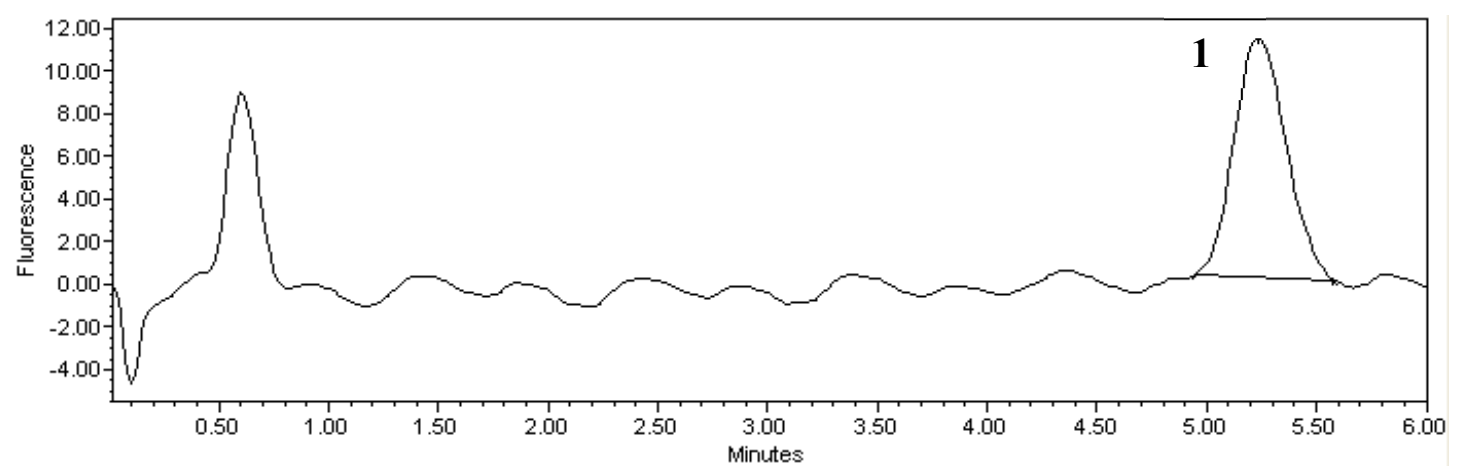

Where cotton was once whitened and sterilized by the application of bleach, now it is commonly whitened and sterilized by treatment with hydrogen peroxide. However, such treatment may not be apparent from an examination of the packaging material which contains the cotton. As previously discussed, only the bags of organic cotton indicated that they had been whitened with hydrogen peroxide; the bags of non-organic cotton were not marked as having been treated with hydrogen peroxide. This made it difficult to locate a source of cotton which had not been previously treated with hydrogen peroxide. In fact, the only sourfce of peroxide-free cotton which could be located was found in the storage room of the FBI Laboratory Research Unit. This cotton was not marked with any manufacturer's information, making it impossible to locate additional sources of this material. Swatches of cotton which were cut from the roll were free of hydrogen 
peroxide; the results of analysis of a water extract of this material by HPLC/ED in DC mode and HPLC/FD are presented in Figures 7.3 and 7.4.

Figure 7.3. Analysis of a cotton swatch from a roll of cotton by HPLC/ED in DC mode. Conditions as in Figure 7.1. Peak 1, unknown, Rt 1.501 min. Unlike the pre-packaged cotton swabs, cotton swatches cut from this source were free of hydrogen peroxide.

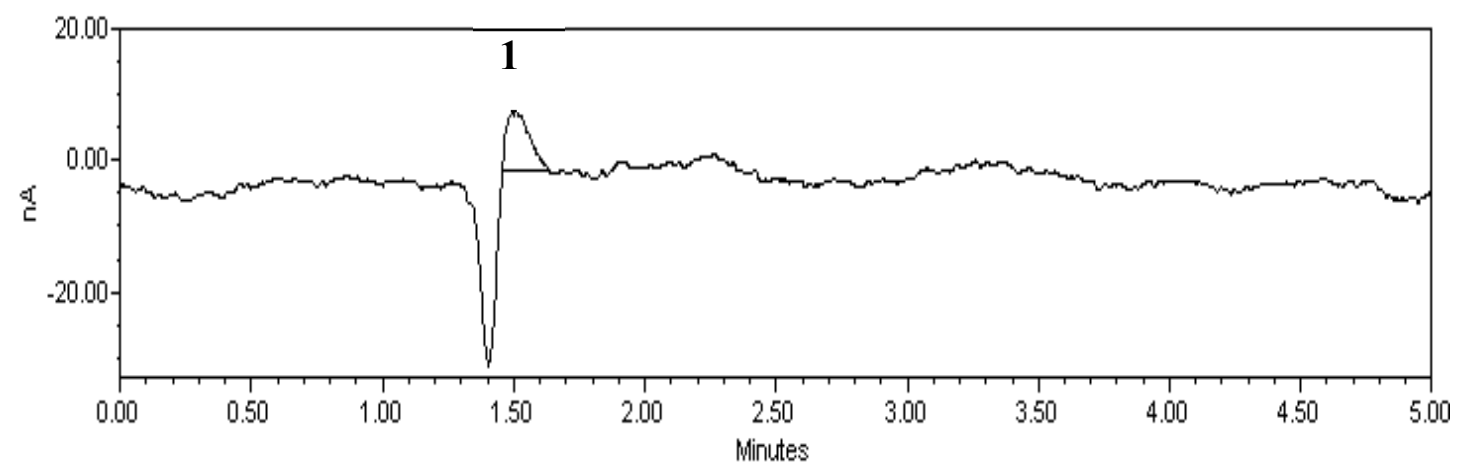

Figure 7.4. Analysis of a cotton swatch from a roll of cotton by HPLC/FD. Conditions as in Figure 7.2. Unlike the pre-packaged cotton swabs, cotton swatches cut from this source were free of hydrogen peroxide.

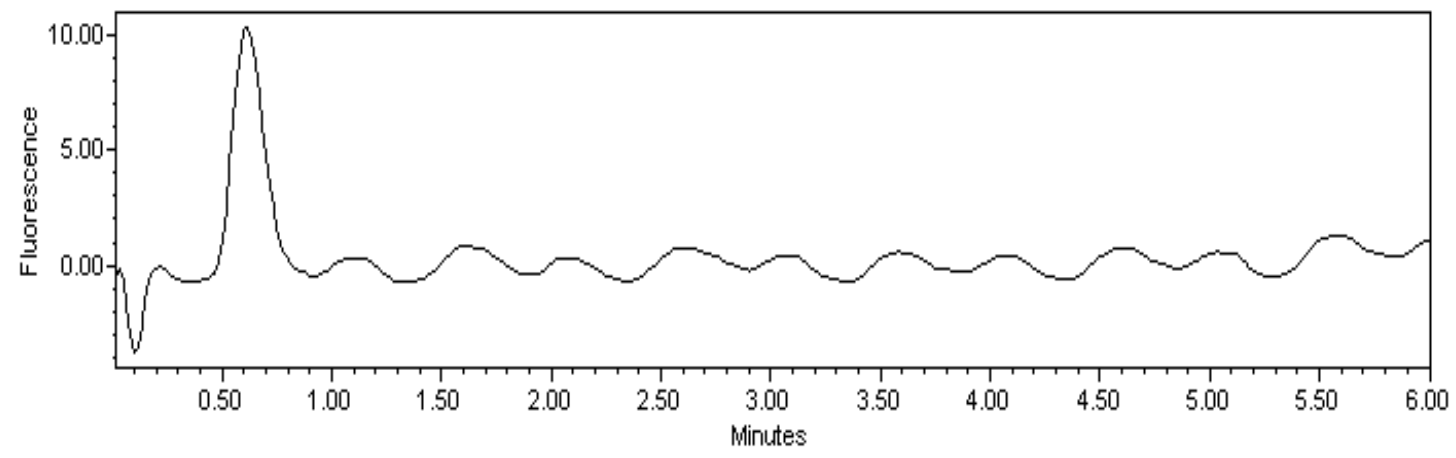


Because all of the commercially available cotton samples were found to contain hydrogen peroxide, additional synthetic and non-synthetic materials were tested to determine their suitability for the collection of hydrogen peroxide residues. The tested materials included brown paper towels, lab wipes, filter paper, and nylon filters. Given that hydrogen peroxide was detected in six of the seven types of white cotton which were analyzed, it was hypothesized that the white cellulose-type samples would also have been treated with hydrogen peroxide, and peroxide residues were likely to be present.

Extracts from each type of sample were analyzed on the electrochemical system, and the presence of hydrogen peroxide was confirmed on the fluorescence system. Three point calibration curves were generated on the HPLC/ED system in DC mode so that the amount of hydrogen peroxide present in each sample could be determined. The results of these analyses are presented in Table 7.5. Based on the results of this experiment, two additional substrates were tested to determine if they contained detectable levels of hydrogen peroxide: white printer paper and toilet paper. The results of these analyses are also presented in Table 7.5.

It is apparent from viewing Table 7.5 that hydrogen peroxide was present in all of the white non-cotton samples which were evaluated, with the exception of the nylon filters and the filter paper. Therefore, the proposed hypothesis that hydrogen peroxide would be detected in all of the white non-cotton samples was incorrect. Surprisingly, not only was hydrogen peroxide present in the samples of brown paper towel which were analyzed, but this substrate had one of the highest values for $\mu \mathrm{g}$ of hydrogen peroxide per gram of substrate at 0.92 . For this type of material, hydrogen peroxide may have been employed as an anti-microbial agent, rather than a whitening agent. 
Table 7.5. Results of analysis of additional media. Peroxide residues were extracted from the substrates with water. Standard HPLC/FD and HPLC/ED in DC mode analysis conditions were applied. The amount of hydrogen peroxide present in each substrate was determined by HPLC/ED in DC mode. Hydrogen peroxide was present in four of the six substrates which were analyzed.

\begin{tabular}{|c|c|c|c|c|}
\hline $\begin{array}{c}\text { Material } \\
(n=3)\end{array}$ & $\begin{array}{c}\text { Ave amount of } \mathrm{H}_{2} \mathrm{O}_{2} \\
\text { per substrate }\end{array}$ & $\begin{array}{c}\text { Stnd Dev } \\
\mathrm{H}_{2} \mathrm{O}_{2}\end{array}$ & $\begin{array}{c}\text { Ave mass } \\
\text { of } \\
\text { substrate }\end{array}$ & $\begin{array}{c}\mu \mathrm{g} \mathrm{H}_{2} \mathrm{O}_{2} \text { per } \\
\text { gram of } \\
\text { substrate }\end{array}$ \\
\hline White Printer Paper & $0.32 \mu \mathrm{g}$ & $\pm 0.11 \mu \mathrm{g}$ & $0.075 \mathrm{~g}$ & 4.27 \\
\hline Brown Paper Towel & $0.27 \mu \mathrm{g}$ & $\pm 0.03 \mu \mathrm{g}$ & $0.293 \mathrm{~g}$ & 0.92 \\
\hline Laboratory Wipe & $0.11 \mu \mathrm{g}$ & $\pm 0.01 \mu \mathrm{g}$ & $0.209 \mathrm{~g}$ & 0.51 \\
\hline Toilet Paper & $0.12 \mu \mathrm{g}$ & $\pm 0.01 \mu \mathrm{g}$ & $0.275 \mathrm{~g}$ & 0.44 \\
\hline Filter Paper & $0 \mu \mathrm{g}$ & $\pm 0 \mu \mathrm{g}$ & $0.960 \mathrm{~g}$ & 0 \\
\hline Nylon Filter & $0 \mu \mathrm{g}$ & $\pm 0 \mu \mathrm{g}$ & $0.105 \mathrm{~g}$ & 0 \\
\hline
\end{tabular}

Cleaning protocols for the removal of hydrogen peroxide from the cotton swabs were explored. Following published protocols for the preparation of cotton swabs for the collection of high explosives residue (36) (40), a combination of water and isopropanol washes was applied to a group of thirty Johnson \& Johnson cotton balls to remove hydrogen peroxide from the cotton, as extracts from two of these cotton balls gave no signal for hydrogen peroxide on either analytical system. This group of cotton was later used for sampling of post-blast debris from the September 2008 field tests. 
Because a limited supply of Johnson \& Johnson cotton balls was available, additional brands of cotton balls were evaluated. Testing of blank, untreated CVS Regular cotton balls resulted in an average recovery of $0.21 \mu \mathrm{g}$ of hydrogen peroxide (see Table 7.4). A group of twenty CVS Regular cotton balls were subjected to the same washing procedure as the Johnson \& Johnson cotton balls, with the exception that the CVS Regular cotton balls were dried in a vacuum oven at $\mathrm{T} \sim 78^{\circ} \mathrm{C}$ for twenty-four hours. Extracts from two of these cotton swabs did not yield a signal for hydrogen peroxide on the fluorescence system, but did yield a very small signal for hydrogen peroxide on the electrochemical system. This peroxide signal was right at the limit of detection for hydrogen peroxide at the largest injection volume $(100 \mu \mathrm{L})$ that could be used. When a $50 \mu \mathrm{L}$ injection volume was used, the hydrogen peroxide signal was below the limit of detection.

In an attempt to remove all traces of hydrogen peroxide from the cotton balls, this group of cotton was washed again with additional water and isopropanol, and dried in the vacuum oven at $\mathrm{T} \sim 78^{\circ} \mathrm{C}$ for another twenty-four hours. Extracts from these cotton balls resulted in the absence of a peroxide signal on the fluorescence system, but the same very small peroxide signal on the electrochemical system. Again, this peroxide signal was right at the limit of detection for hydrogen peroxide at the largest injection volume that could be used. To determine whether this remaining hydrogen peroxide could be driven out of the cotton with the application of more heat, the same group of twice-cleaned cotton balls were heated in the vacuum oven for another seventy-two hours at $\mathrm{T} \sim 100^{\circ} \mathrm{C}$. When the cotton was removed from the vacuum oven, a brown, charred appearance was noted. Analysis of a water extract from one of these cotton balls resulted in the absence 
of a peroxide signal on both the fluorescence and electrochemical systems. However, a large peak was present on the chromatogram from the electrochemical system at 1.69 minutes. It is theorized that this peak is the result of decomposition of the cotton fibers in response to exposure to heat. Heating of cotton swabs in the vacuum oven at $\mathrm{T} \sim 78^{\circ} \mathrm{C}$ for seventy-two hours without the prior application of water/isopropanol washes failed to completely remove the hydrogen peroxide.

At this time, the most promising procedure for the removal of hydrogen peroxide from cotton balls appears to be a series of isopropanol/water washes. However, this procedure did not completely remove all of the hydrogen peroxide from both brands of cotton. Further research is needed at this point to develop a protocol for the complete removal of hydrogen peroxide from cotton. Further experiments with drying the cotton in a vacuum oven should be explored. Ideally, a supplier of cotton which has not been treated with hydrogen peroxide should be located for use in the collection of case-work samples.

\section{Survey of Law Enforcement Training Facility}

It is known that hydrogen peroxide is naturally present in air and water (159).

Francois et al. (141) measured an average hydrogen peroxide concentration of $0.15 \mathrm{nM}$ in air, while Hellpointner and Gaeb (142) measured an average hydrogen peroxide concentration of $0.7 \mu \mathrm{M}$ in melted snow, $3.5 \mu \mathrm{M}$ in rain, and $0.4 \mathrm{nM}$ in air. Currently, it is unknown whether or not hydrogen peroxide exists on surfaces in indoor settings at high enough levels that it can be detected given the limit of detection of the present methods. Because indoor settings are protected from exposure, and because the amount of 
hydrogen peroxide present in the air is low, the presence of hydrogen peroxide in indoor settings may not be significant. However, hydrogen peroxide is present in certain cleaning agents which are utilized in indoor settings. Residues from these solutions could confuse data interpretation if present on surfaces which are sampled for post-blast residue collection.

To explore this issue, a small-scale survey of a law enforcement training facility was conducted. Thirty-four high traffic areas such as banisters, floors, railings, telephones, computer keyboards, and door handles were sampled using dry cotton swatches known to be free of hydrogen peroxide. It was hypothesized that hydrogen peroxide residues were likely to be present on surfaces subject to regular treatment with cleaning products, such as the break room table and the bathroom counter. Surfaces which were not subject to regular treatment with cleaning products, such as hallway railings and door handles, would be less likely to yield hydrogen peroxide residues. Water extracts from these swabs were filtered and analyzed on the electrochemical and fluorescence systems. Three point calibration curves were generated on the HPLC/ED system in DC mode so that the amount of hydrogen peroxide present in each sample could be determined. The results of this study are presented in Table 7.6.

Only three of the thirty-four samples yielded a signal for hydrogen peroxide: a laboratory tool box $\left(0.05 \mu \mathrm{g} / \mathrm{mL} \mathrm{H}_{2} \mathrm{O}_{2}\right)$, a toilet paper dispenser in the women's restroom $\left(0.09 \mu \mathrm{g} / \mathrm{mL} \mathrm{H}_{2} \mathrm{O}_{2}\right)$, and a wooden coffee table in a common area $\left(0.90 \mu \mathrm{g} / \mathrm{mL} \mathrm{H}_{2} \mathrm{O}_{2}\right)$. The locations where hydrogen peroxide was detected appeared to be somewhat random, and not in keeping with the proposed hypothesis. At this time, the only conclusion which 
can be drawn is that hydrogen peroxide may be present in indoor settings, which could complicate the analysis of a post-blast scene.

Table 7.6. Results of survey of a law enforcement training academy. Hydrogen peroxide was detected by HPLC/ED in DC Mode in three of the thirty-four areas which were sampled.

\begin{tabular}{|c|c|c|}
\hline Sample & $\begin{array}{c}\mathrm{H}_{2} \mathrm{O}_{2} \text { on } \mathrm{HPLC} / \mathrm{ED} \text { in } \\
\text { DC Mode }\end{array}$ & $\mathrm{H}_{2} \mathrm{O}_{2}$ on $\mathrm{HPLC} / F \mathrm{D}$ \\
\hline Blank Cotton (2) & No & No \\
\hline Bathroom Counter (1) & No & No \\
\hline Bathroom Floor Tile (1) & No & No \\
\hline Plastic Toilet Paper Dispenser (1) & $0.09 \mu \mathrm{g} / \mathrm{mL}$ & $<$ LOD \\
\hline Plastic Paper Towel Dispenser (1) & No & No \\
\hline Metal Elevator Buttons (1) & No & No \\
\hline Plastic Telephone Handset (1) & No & No \\
\hline Metal Door Plate (1) & No & No \\
\hline Water Fountain Metal Handle (2) & No & No \\
\hline Wooden Break Room Table (1) & No & No \\
\hline Wooden Loft Table (1) & No & No \\
\hline Concrete Wall of Hallway (1) & No & No \\
\hline Hallway Floor Tile (4) & No & No \\
\hline Plastic/Metal Computer (1) & No & No \\
\hline Metal Door Handle (3) & No & No \\
\hline Metal Refrigerator Handle (1) & No & No \\
\hline Metal Tool Chest (1) & $0.05 \mu \mathrm{g} / \mathrm{mL}$ & $<$ LOD \\
\hline Hood (1) & No & No \\
\hline Laboratory Bench Top (1) & No & No \\
\hline Brick Stairs (1) & No & No \\
\hline Wooden Stairway Railing (1) & No & No \\
\hline Metal Railing (1) & No & No \\
\hline ATM Machine Metal Buttons (1) & No & No \\
\hline Chair Fabric (1) & No & No \\
\hline Vending Machine Plastic Buttons (1) & No & No \\
\hline Leather Couch (1) & No & No \\
\hline Gymnasium Rubber Mats (2) & No & No \\
\hline Wooden Coffee Table (1) & $0.90 \mu \mathrm{g} / \mathrm{mL}$ & Yes \\
\hline
\end{tabular}




\section{E. Analysis of Post-Blast Debris from Mixtures of Concentrated Hydrogen Peroxide and Nitromethane}

A series of controlled detonations of large-scale mixtures of concentrated hydrogen peroxide/nitromethane were conducted in October and November of 2006 by FBI scientists and contractors. Post-blast materials from these tests which were collected included plastic debris, metal debris, cotton swabs from large pieces of debris, and soil samples from the area in which the explosion occurred. The color and texture of the plastic debris was consistent with a plastic barrel which contained the improvised explosive mixture prior to detonation. The source of the metal debris was most likely the van in which the hydrogen peroxide/fuel mixture was detonated.

It was hypothesized that because the concentrated hydrogen peroxide had been contained within a plastic barrel prior to detonation of the improvised mixture, the plastic debris fragments would be more likely to yield hydrogen peroxide residues than the metal automobile fragments, soil samples, or cotton swabs. Extracts from each piece of debris were analyzed by HPLC/FD and HPLC/ED in PAD mode. Because it is not standard practice at the FBI Laboratory Explosives Unit to quantify post-blast explosives residues, the level of hydrogen peroxide present on post-blast debris was not quantified (the HPLC/ED and HPLC/FD analyses were performed as qualitative analyses only). The results of the analysis of all the materials collected from this test site are presented in Table 7.7. The results from the analysis of one of the pieces of metal debris are presented in Figures 7.5 and 7.6. While hydrogen peroxide is clearly present in both chromatograms, there are additional peaks of an unknown nature which are also present. 
Table 7.7. Results of analysis of post-blast debris from a large scale detonation of hydrogen peroxide/nitromethane. Hydrogen peroxide residues were detected on the metal debris fragments only.

\begin{tabular}{|c|c|c|c|}
\hline Material & $\begin{array}{c}\text { Number of samples } \\
\text { analyzed }\end{array}$ & $\begin{array}{c}\mathrm{H}_{2} \mathrm{O}_{2} \text { by HPLC/ED } \\
\text { in PAD Mode }\end{array}$ & $\mathrm{H}_{2} \mathrm{O}_{2}$ by HPLC/FD \\
\hline Plastic Fragments & 10 & No (10) & No (10) \\
\hline Metal Fragments & 4 & Yes (4) & Yes (4) \\
\hline Soil Samples & 5 & No (5) & No (9) \\
\hline Cotton Swabs & 9 & No (9) & \\
\hline
\end{tabular}

Figure 7.5. Analysis of a metal debris fragment by HPLC/ED in PAD mode. Conditions: Dionex CarboPac PA10 analytical column, isocratic elution with $150 \mathrm{mM} \mathrm{NaOH} / 150$ $\mathrm{mM} \mathrm{NaOAc}$, flow rate $1 \mathrm{~mL} / \mathrm{min}, 10 \mu \mathrm{L}$ injection, $\mathrm{PAD}$ at $45^{\circ} \mathrm{C}\left(\mathrm{E}_{1}={ }^{+} 0.01 \mathrm{~V}, t_{l}=300 \mathrm{~ms}\right.$, $\left.t_{s}=100 \mathrm{~ms}, \mathrm{E}_{2}={ }^{+} 0.65 \mathrm{~V}, t_{2}=150 \mathrm{~ms}, \mathrm{E} 3={ }^{-} 0.65 \mathrm{~V}, t_{3}=150 \mathrm{~ms}\right)$. Peak 1, unknown, Rt 1.377; Peak 2, hydrogen peroxide, Rt $2.176 \mathrm{~min}$. Hydrogen peroxide was detected on the metal fragment despite storage of the debris at room temperature for $\sim 12$ months prior to analysis.

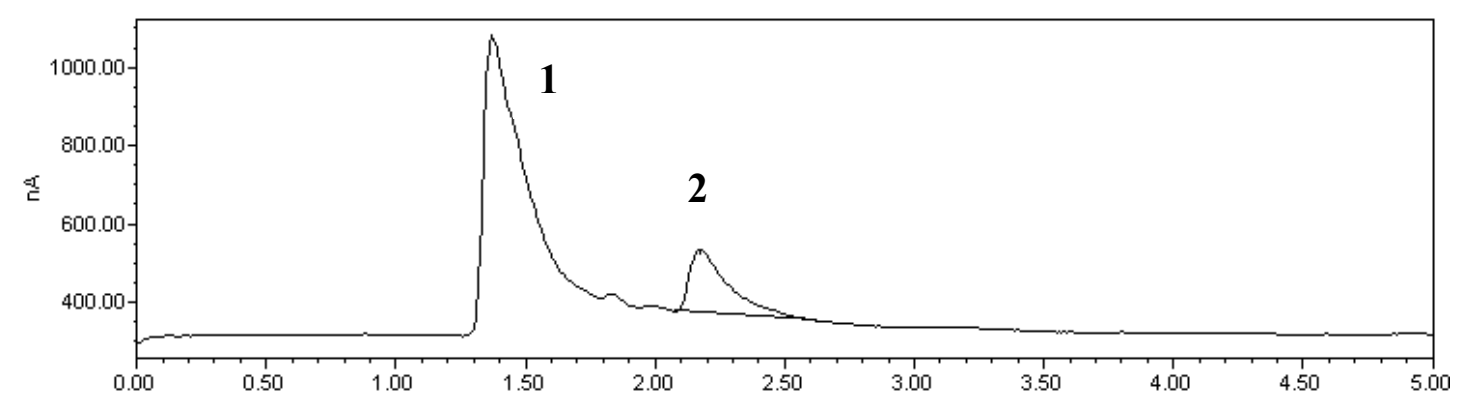


Figure 7.6. Analysis of a metal debris fragment by HPLC/FD. Conditions: Dionex C-18 analytical column, mobile phase: $1 \times 10^{-3} \mathrm{M} \mathrm{H}_{2} \mathrm{SO}_{4} / 1 \times 10^{-4} \mathrm{M}$ EDTA at flow rate 0.6 $\mathrm{mL} / \mathrm{min}, 20 \mu \mathrm{L}$ injection, reagent $8 \mu \mathrm{M}$ hemin $/ 80 \mu \mathrm{M}$ POPHA in ammonia buffer $\mathrm{pH} 9.5$ at flow rate $0.2 \mathrm{~mL} / \mathrm{min}$, post-column reactor $5 \mathrm{~m}$, base $100 \mathrm{mM} \mathrm{NaOH}$ at flow rate 0.25 $\mathrm{mL} / \mathrm{min}, \lambda_{\mathrm{ex}}=320 \mathrm{~nm}, \lambda_{\mathrm{em}}=405 \mathrm{~nm}$. Peak 1, unknown, Rt 4.639 minutes; Peak 2 , hydrogen peroxide, Rt 5.060 minutes. Hydrogen peroxide was detected on the metal fragment despite storage of the debris at room temperature for $\sim 12$ months prior to analysis.

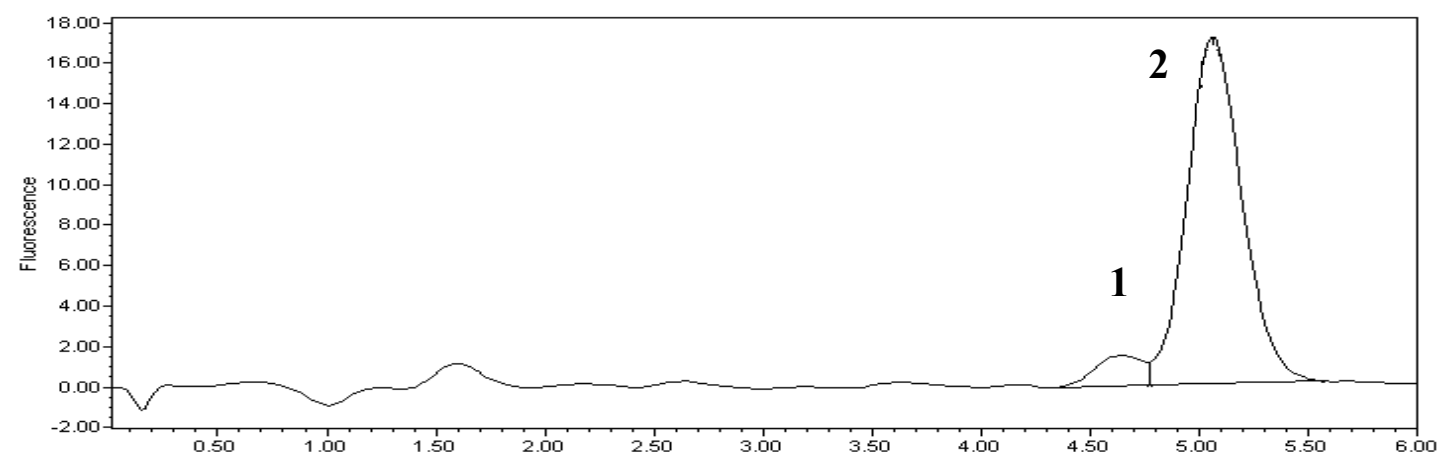

It was hypothesized that because the hydrogen peroxide had been contained within the plastic barrel prior to the shot, the plastic debris fragments would be more likely to yield hydrogen peroxide residues than the metal fragments, soil samples, or cotton swabs. In viewing Table 7.7, it is apparent that this was not the case. Of the ten pieces of plastic debris which were analyzed, not one yielded a signal for hydrogen peroxide by either analytical method. The soil samples and cotton swabs were also negative for hydrogen peroxide residues. In contrast, all four pieces of metal debris yielded a signal for hydrogen peroxide. The reason for this discrepancy may lie in the 
way in which the materials were packaged and stored. The soil samples were packaged in plastic screw-cap containers which were not air-tight. The cotton swabs were packaged in glass screw-top vials. All of the plastic debris was packaged together in a single layer of gallon-sized plastic Ziploc-style bag. All of the metal debris was packaged in the same way. These materials were stored at room temperature for approximately one year prior to analysis. It is possible that during this time period, the seals on the Ziploc-style bag which held the plastic debris were disturbed, thereby allowing any hydrogen peroxide residues which were initially present on the surface of the plastic to evaporate. A similar issue may have occurred with the soil samples and the cotton swabs.

During the summer and fall of 2007, an additional series of tests were performed by the FBI on small-scale mixtures of concentrated hydrogen peroxide with a variety of fuel sources. Twenty-four steel witness plates which had been exposed to the detonation of these mixtures were packaged in heat-sealed nylon bags and stored at room temperature for up to twelve months prior to analysis. Of the twenty-four witness plates which had been exposed to hydrogen peroxide, fifteen plates tested positive for hydrogen peroxide by HPLC/FD analysis. Unfortunately, the HPLC/ED analysis of the plates in PAD mode was hindered by the presence of high levels of background material, to the extent that the presence of hydrogen peroxide initially could not be confirmed on any of the plates (even the "blank" plates). These results are presented in Table 7.8. Chromatograms from the analysis of one of these witness plates by HPLC/FD and HPLC/ED in PAD mode are presented in Figures 7.7 and 7.8. Analysis of the chromatogram from the HPLC/ED in PAD mode analysis reveals the presence of four peaks, not one of which corresponds to the retention time of hydrogen peroxide for this 
method (2.25 minutes). Because of the strong signal for the unknown peaks, the presence of the hydrogen peroxide signal is inconclusive. It is unknown how the steel plates were treated or cleaned prior to use as witness plates. It should be noted that when the witness plates were later cleaned with water/methanol washes, extracts from the plates were free of extraneous material.

Table 7.8. Results of analysis of witness plates. Hydrogen peroxide was detected by HPLC/FD on fifteen of the twenty-four plates, but the presence of peroxide could not be confirmed due to the presence of interfering components in the HPLC/ED chromatograms.

\begin{tabular}{|c|c|c|c|}
\hline Witness Plates & $\begin{array}{c}\mathrm{H}_{2} \mathrm{O}_{2} \text { by HPLC/ED in } \\
\text { PAD Mode }\end{array}$ & \multicolumn{2}{|l|}{$\mathrm{H}_{2} \mathrm{O}_{2}$ by HPLC/FD } \\
\hline Blank (4) & Obscured (4) & No (4) & Yes (0) \\
\hline Neat hydrogen peroxide (2) & Obscured (2) & No (1) & Yes (1) \\
\hline Hydrogen peroxide / fuel \#1 (2) & Obscured (2) & No (0) & Yes (2) \\
\hline Hydrogen peroxide / fuel \#2 (2) & Obscured (2) & No (1) & Yes (1) \\
\hline Hydrogen peroxide / fuel \#3 (2) & Obscured (2) & No (0) & Yes (2) \\
\hline Hydrogen peroxide / fuel \#4 (4) & Obscured (4) & No (1) & Yes (3) \\
\hline Hydrogen peroxide / fuel \#5 (2) & Obscured (2) & No (1) & Yes (1) \\
\hline Hydrogen peroxide / fuel \#6 (2) & Obscured (2) & No (0) & Yes (2) \\
\hline Hydrogen peroxide / fuel \#7 (2) & Obscured (2) & No (0) & Yes (2) \\
\hline Hydrogen peroxide / fuel \#8 (2) & Obscured (2) & No (1) & Yes (1) \\
\hline
\end{tabular}


Figure 7.7. Analysis of a metal witness plate exposed to concentrated hydrogen peroxide/fuel \#1 by HPLC/FD. Conditions as in Figure 7.6. Peak 1, hydrogen peroxide, Rt 5.003 min. Other than the hydrogen peroxide peak, no additional peaks are present in this chromatogram.

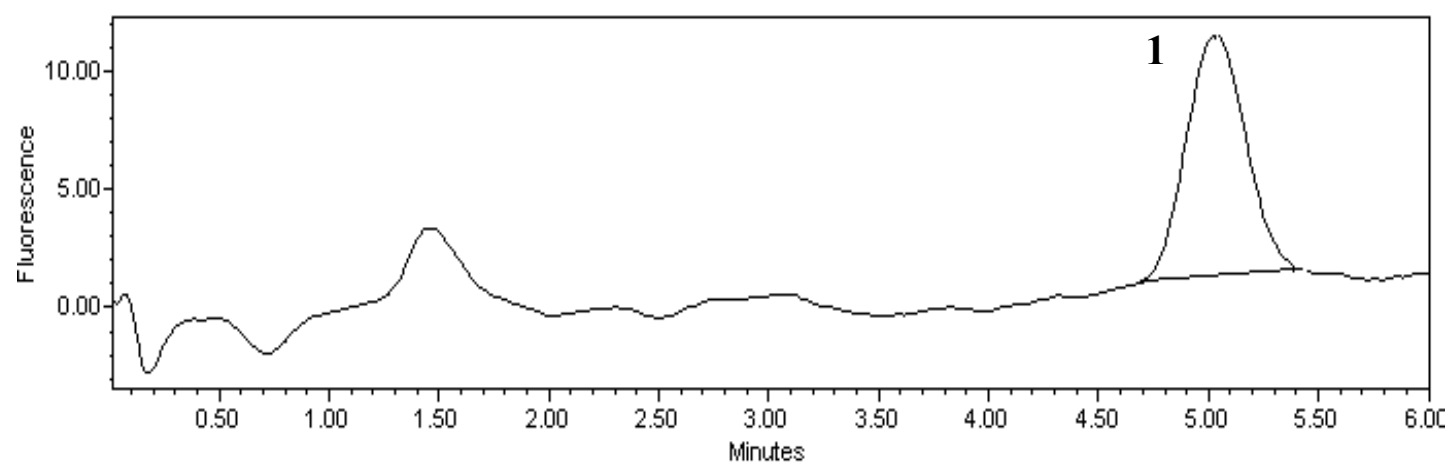

Figure 7.8. Analysis of a metal witness plate exposed to concentrated hydrogen peroxide/fuel \#1 by HPLC/ED in PAD mode. Conditions as in Figure 7.5. Peak 1, unknown, Rt 1.478 min; Peak 2, unknown, Rt 1.862 min; Peak 3, unknown, Rt 2.190 min; Peak 4, unknown, Rt 2.428 min. Hydrogen peroxide elutes at 2.19 minutes. The presence of a co-eluting peak at 2.19 minutes (peak \#3) hinders the determination of whether or not hydrogen peroxide is present.

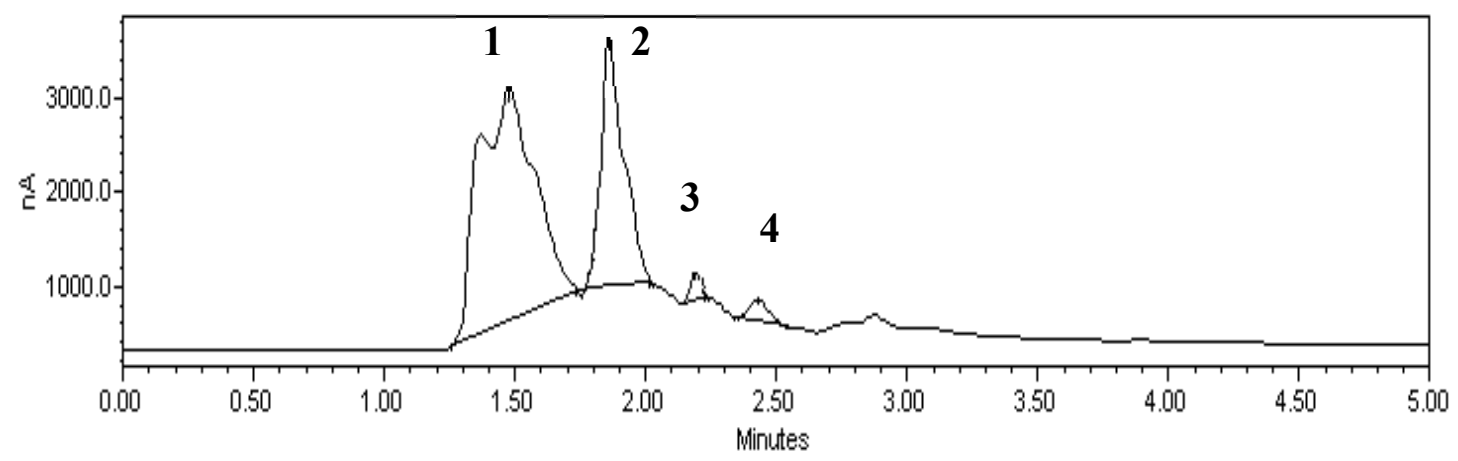


It appeared that PAD mode contributed to the presence of background material in the chromatograms, most likely through the generation of electroactive species. The problem of interfering material in the HPLC/ED method in PAD mode led to the reoptimization of the separation and detection parameters for this system. The re-optimized system included a CarboPac PA10 analytical column and a mobile phase which was 150 $\mathrm{mM} \mathrm{NaOAc}$ at $\mathrm{pH} 10.5$ with detection in DC mode at an applied voltage of $+400 \mathrm{mV}$. When five of the witness plate extracts which had tested positive for hydrogen peroxide by HPLC/FD were re-tested by HPLC/ED in DC mode several months later, co-elution of background components with hydrogen peroxide was no longer a problem. Data from a plate extract re-analyzed by HPLC/ED in DC mode is presented in Figure 7.9.

Figure 7.9. Analysis of a metal witness plate exposed to concentrated hydrogen peroxide/fuel \#1 by HPLC/ED in DC mode. For conditions, see text. Peak 1, hydrogen peroxide, Rt 1.923 min. Unlike the chromatogram obtained from analysis of this extract by HPLC/ED in PAD mode, this chromatogram is free of interfering peaks. The hydrogen peroxide peak is unobstructed.

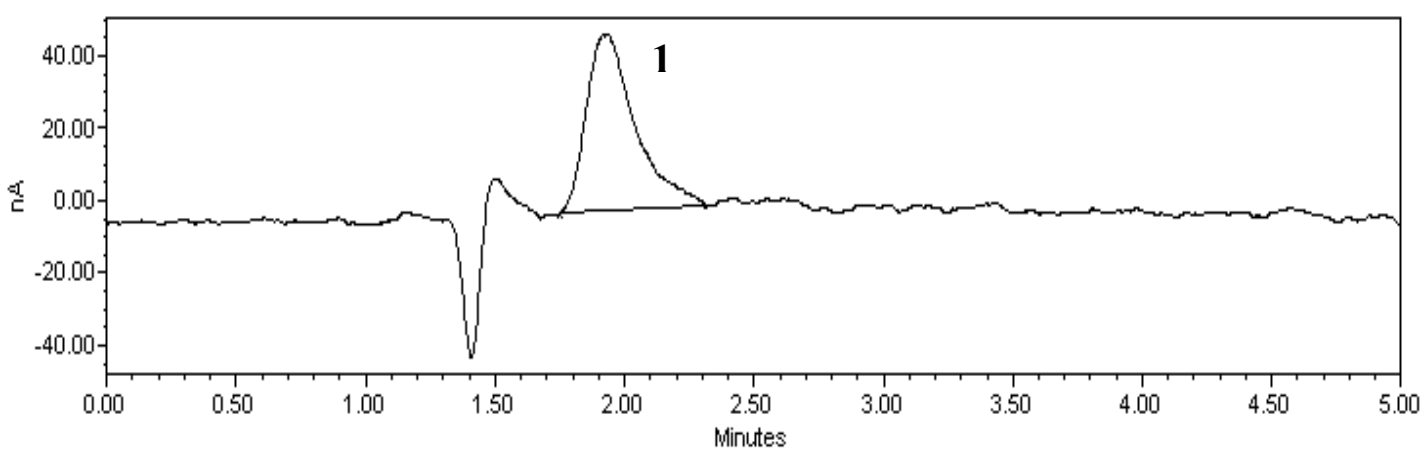


The results from the re-analysis of all five witness plate extracts are presented in Table 7.9. Although the chromatograms for all five extracts were free of extraneous peaks, only two samples yielded a hydrogen peroxide signal. Despite having refrigerated the witness plate extracts at $-4^{\circ} \mathrm{C}$ during the time period between the initial and subsequent analyses, the hydrogen peroxide in three of the extracts degraded to the point where it could no longer be detected.

Table 7.9. Results of re-analysis of five witness plate extracts. During the original analysis, fifteen witness plate extracts were positive for hydrogen peroxide by HPLC/FD, but hydrogen peroxide was obscured by interfering components in the HPLC/ED chromatograms. Re-analysis of five of the witness plate extracts several months later resulted in successful detection of hydrogen peroxide by HPLC/ED in DC mode in two of the five plates.

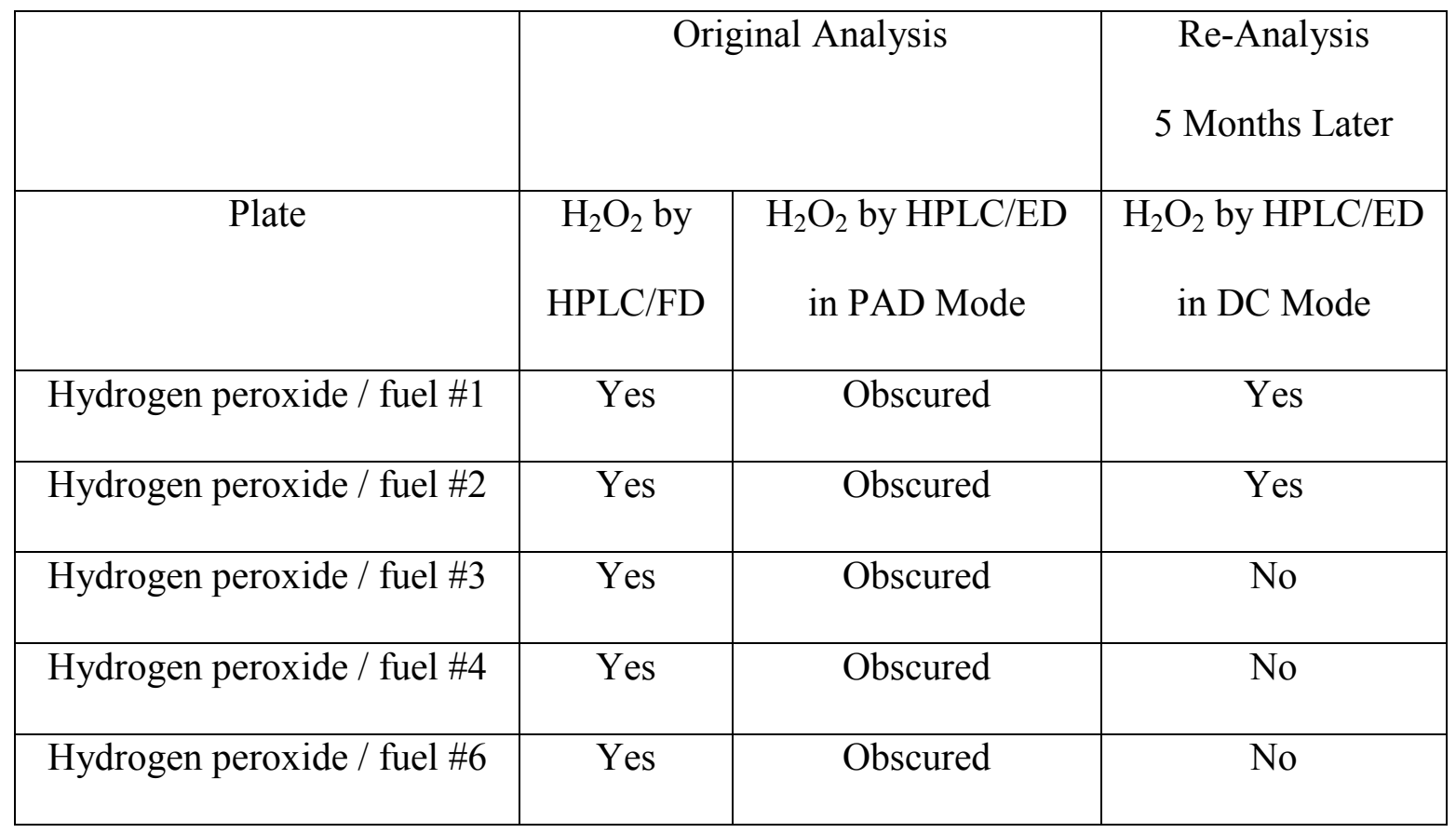


A field test was performed in September of 2008 to evaluate the ability of the optimized methods to detect post-blast hydrogen peroxide residues on a variety of substrates. The test involved the detonation of a small-scale mixture of hydrogen peroxide/nitromethane which was contained inside of a metal ammunition can. Post-blast materials from these tests were collected, including plastic remnants, metal debris, witness plates, and soil samples from the area directly under the explosion. The results of the analysis of the ammunition can fragments, plastic debris, soil samples, and witness plates are presented in Table 7.10. Because it is not standard practice at the FBI Laboratory Explosives Unit to quantify post-blast explosives residues, the level of hydrogen peroxide present on this set of post-blast debris was not quantified. The electrochemical and fluorescence analyses were performed as qualitative analyses only. Samples were reported as positive if the signal strength of hydrogen peroxide was greater than the limit of detection of $0.02 \mu \mathrm{g} / \mathrm{mL}$ for the HPLC/ED system, and $0.19 \mu \mathrm{g} / \mathrm{mL}$ for the HPLC/FD system.

In viewing Table 7.10, it is apparent that hydrogen peroxide was detected in all three sample types by HPLC/FD. Unfortunately, these results could not be confirmed by HPLC/ED in PAD mode because of problems with co-eluting materials which masked the hydrogen peroxide peaks. The separation and detection parameters for the electrochemical method were ultimately determined to be unsuitable for the analysis of post-blast debris because of this problem. Therefore, the separation and detection parameters for this system were re-optimized as previously discussed. The re-optimized system included a CarboPac PA10 analytical column and a mobile phase which was 150 $\mathrm{mM} \mathrm{NaOAc}$ at $\mathrm{pH} 10.5$ with detection in DC mode at an applied voltage of $400 \mathrm{mV}$. 
When the same soil samples and additional ammunition can fragments and plastic debris from this field test were analyzed by HPLC/ED in DC mode, co-elution of background components with hydrogen peroxide was no longer a problem: hydrogen peroxide could be clearly distinguished. No additional peaks were noted in the chromatograms from either analytical system. The results of these analyses are presented in Table 7.11.

Table 7.10. Results of initial analysis of materials collected from a small scale detonation of hydrogen peroxide/nitromethane inside of an ammo can. Hydrogen peroxide residues were detected on the ammunition can fragments, the witness plates, and the soil samples by HPLC/FD. The presence of co-eluting materials made confirmation of $\mathrm{H}_{2} \mathrm{O}_{2}$ by HPLC/ED in PAD mode impossible.

\begin{tabular}{|c|c|c|c|}
\hline Material & $\begin{array}{c}\mathrm{H}_{2} \mathrm{O}_{2} \text { by HPLC/ED in PAD } \\
\text { Mode }\end{array}$ & \multicolumn{2}{|l|}{$\mathrm{H}_{2} \mathrm{O}_{2}$ by HPLC/FD } \\
\hline Ammunition Can Fragments (7) & Obscured (7) & No (1) & Yes (6) \\
\hline Witness Plates (3) & Obscured (3) & No (1) & Yes (2) \\
\hline Soil Samples (3) & Obscured (3) & No (1) & Yes (2) \\
\hline
\end{tabular}

It was theorized that because the improvised explosive mixture was contained within the ammunition can, the ammunition can fragments would be more likely to yield hydrogen peroxide residues than the plastic remnants, soil samples, or witness plates. In fact, hydrogen peroxide residues were recovered from all three of the plastic remnants, two out of the three witness plates, and five out of the six ammunition can fragments. Hydrogen peroxide was not recovered from any of the soil samples. 
Table 7.11. Results of secondary analysis of materials collected from a small scale detonation of hydrogen peroxide/nitromethane inside of an ammunition can. Hydrogen peroxide residues were detected on the ammo can fragments and the plastic remnants.

\begin{tabular}{|c|c|c|c|c|}
\hline \multicolumn{2}{|c|}{ Material } & \multicolumn{2}{|l|}{$\mathrm{H}_{2} \mathrm{O}_{2}$ by HPLC/ED in DC Mode } & $\mathrm{H}_{2} \mathrm{O}_{2}$ by HPLC/FD \\
\hline Ammunition Can Fragments (6) & No (1) & Yes (5) & No (1) & Yes (5) \\
\hline Plastic Remnants (3) & No (0) & Yes (3) & No (0) & Yes (3) \\
\hline Soil (3) & No (3) & Yes (0) & No (3) & Yes (0) \\
\hline
\end{tabular}

Data from an extract from one of the plastic remnants are presented in Figures 7.10 and 7.11. Peaks corresponding to hydrogen peroxide can be seen on the chromatograms from the electrochemical and fluorescence systems. A lack of additional peaks or interferences in the chromatograms should be noted.

Figure 7.10. Analysis of a plastic remnant by HPLC/ED in DC mode. Conditions: Dionex CarboPac PA10 analytical column, isocratic elution with $150 \mathrm{mM} \mathrm{NaOAc}$ at pH 10.5, flow rate $1 \mathrm{~mL} / \mathrm{min}, 100 \mu \mathrm{L}$ injection, $\mathrm{T}=45^{\circ} \mathrm{C}$, DC Mode with $\mathrm{E}=+0.4 \mathrm{~V}$. Peak 1 , hydrogen peroxide, Rt 1.949 min. Hydrogen peroxide residues were readily recovered from all of the plastic remnants which were analyzed.

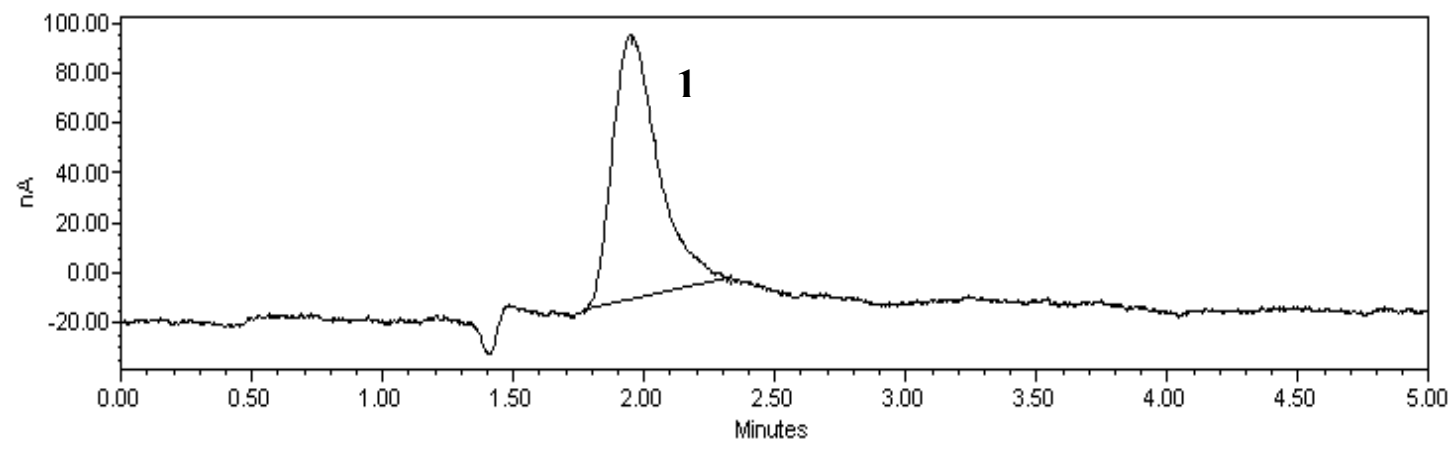


Figure 7.11. Analysis of a plastic remnant by HPLC/FD. Conditions: Dionex C-18 analytical column, isocratic elution with $1 \times 10^{-3} \mathrm{M} \mathrm{H}_{2} \mathrm{SO}_{4} / 1 \times 10^{-4} \mathrm{M}$ EDTA at flow rate $0.6 \mathrm{~mL} / \mathrm{min}, 100 \mu \mathrm{L}$ injection, reagent $8 \mu \mathrm{M}$ hemin/80 $\mu \mathrm{M}$ POPHA in ammonia buffer $\mathrm{pH} 9.5$ at flow rate $0.2 \mathrm{~mL} / \mathrm{min}$, post-column reactor $2 \mathrm{~m}, \mathrm{~T}=25^{\circ} \mathrm{C}$, base $0.1 \mathrm{M} \mathrm{NaOH}$ at flow rate $0.2 \mathrm{~mL} / \mathrm{min}, \lambda_{\mathrm{ex}}=320 \mathrm{~nm}, \lambda \mathrm{e}_{\mathrm{m}}=405 \mathrm{~nm}$. Peak 1 , hydrogen peroxide, Rt 5.195 min. Hydrogen peroxide residues were readily recovered from all of the plastic remnants which were analyzed.

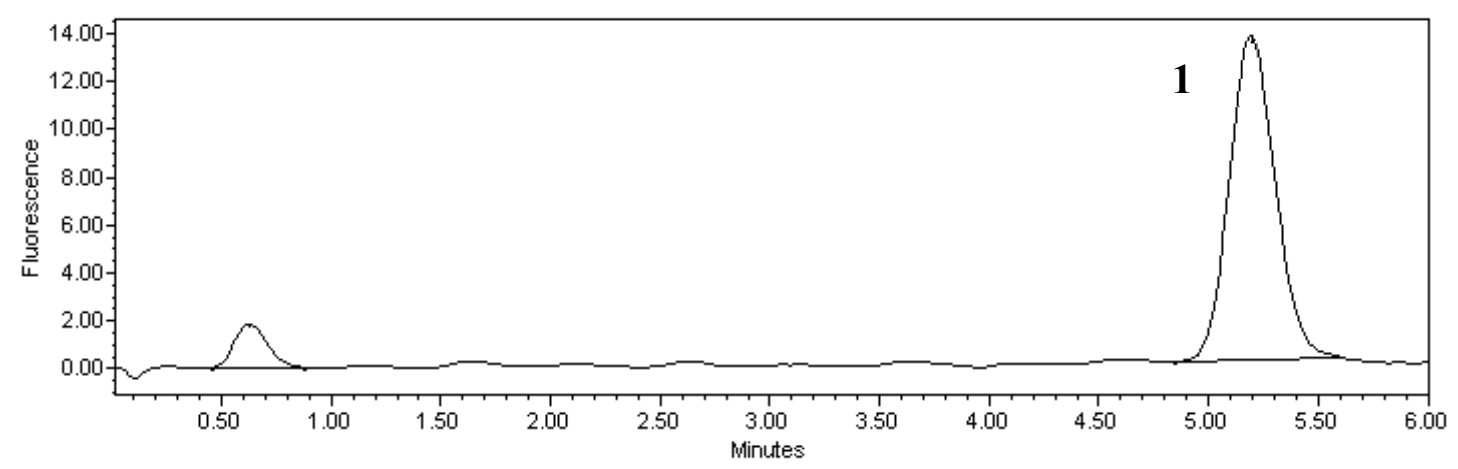

Data from an extract of an ammunition can fragment are presented in Figures 7.12 and 7.13. Peaks corresponding to hydrogen peroxide can be seen on the chromatograms from the electrochemical and fluorescence systems. It should be noted that strength of the hydrogen peroxide signal is approaching the limit of detection for the fluorescence method. The absence of interfering peaks or additional unknown peaks should also be noted. Both techniques demonstrate excellent specificity for the detection of hydrogen peroxide. 
Figure 7.12. Analysis of water rinse of exterior of ammunition can debris by HPLC/ED in DC mode. Conditions: Dionex CarboPac PA10 analytical column, isocratic elution with $150 \mathrm{mM} \mathrm{NaOAc}$ at $\mathrm{pH} 10.5$, flow rate $1 \mathrm{~mL} / \mathrm{min}, 100 \mu \mathrm{L}$ injection, $\mathrm{T}=45^{\circ} \mathrm{C}, \mathrm{DC}$ Mode with E=+0.4V. Peak 1, hydrogen peroxide, Rt 1.940 min. Hydrogen peroxide residues were present on five of the six ammunition can fragments which were analyzed.

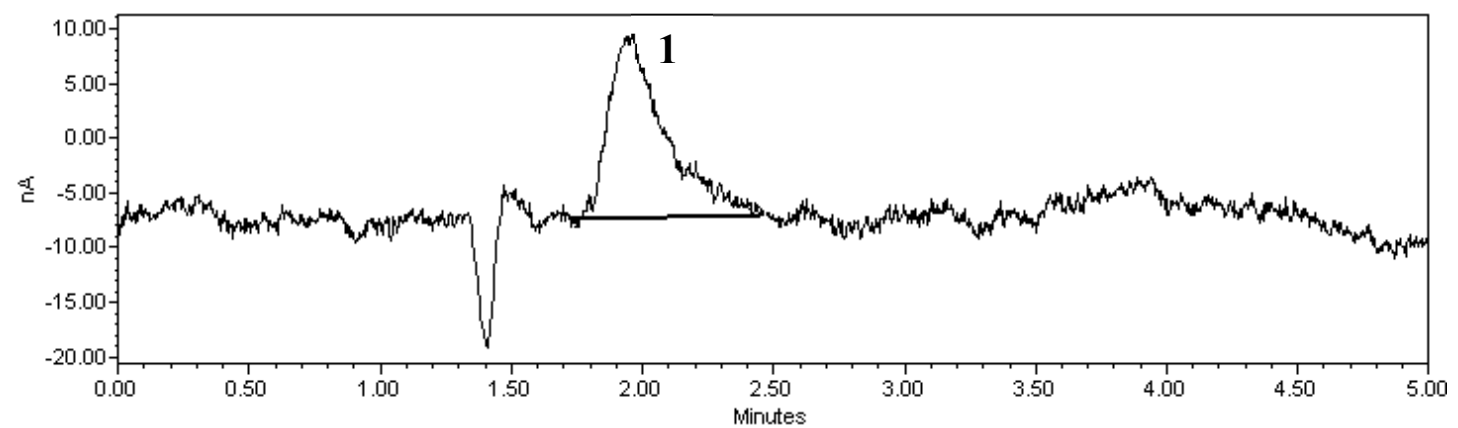

Figure 7.13. Analysis of water rinse of exterior of ammunition can debris by HPLC/FD. Conditions: Dionex C-18 analytical column, isocratic elution with $1 \times 10^{-3} \mathrm{M} \mathrm{H}_{2} \mathrm{SO}_{4} / 1 \times 10^{-}$ ${ }^{4} \mathrm{M}$ EDTA at flow rate $0.6 \mathrm{~mL} / \mathrm{min}, 100 \mu \mathrm{L}$ injection, reagent $8 \mu \mathrm{M}$ hemin $/ 80 \mu \mathrm{M}$ POPHA in ammonia buffer $\mathrm{pH} 9.5$ at flow rate $0.2 \mathrm{~mL} / \mathrm{min}$, post-column reactor $2 \mathrm{~m}$, $\mathrm{T}=25^{\circ} \mathrm{C}$, base $0.1 \mathrm{M} \mathrm{NaOH}$ at flow rate $0.2 \mathrm{~mL} / \mathrm{min}, \lambda_{\mathrm{ex}}=320 \mathrm{~nm}, \lambda_{\mathrm{em}}=405 \mathrm{~nm}$. Peak 1 , hydrogen peroxide, Rt 5.196 min. Hydrogen peroxide residues were present on five of the six ammunition can fragments which were analyzed.

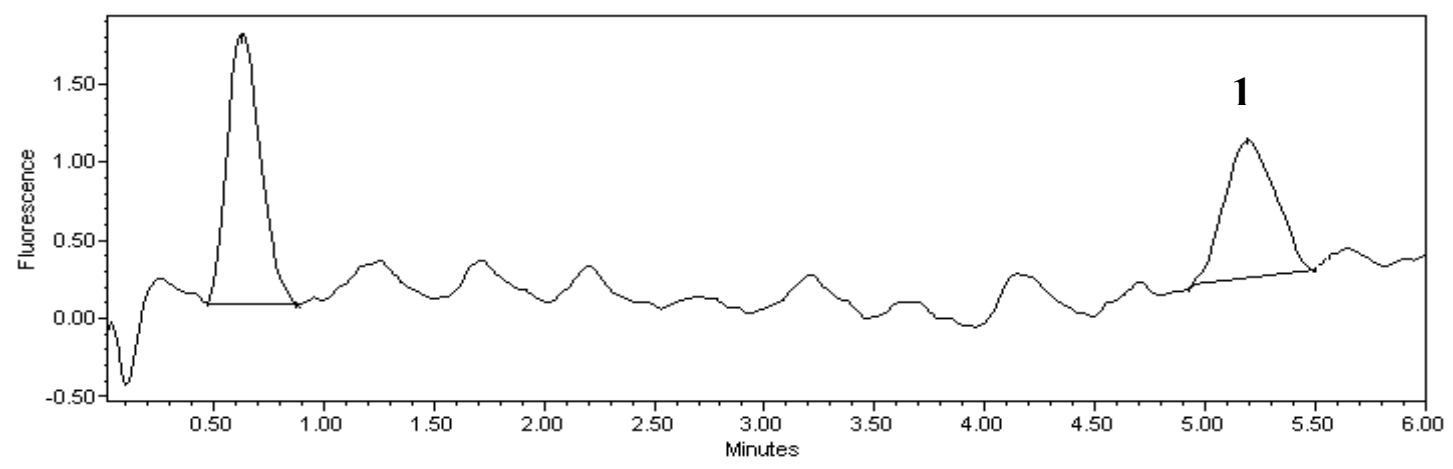


The second test from September 2008 involved the detonation of a small-scale mixture of hydrogen peroxide/nitromethane inside of a car. Post-blast materials collected from the test site included plastic remnants, fabric remnants, glass, witness plates, and cotton swabs utilized in the sampling of debris which was too large to be transported back to the laboratory for analysis. It was theorized that because the improvised explosive mixture was in close proximity to the backseat of the car, the seat fabric and window glass from that area would be the most likely substrates from which hydrogen peroxide residues could be collected. The results of the analysis of the collected debris are presented in Table 7.12. Because it is not standard practice at the FBI Laboratory Explosives Unit to quantify post-blast explosives residues, the level of hydrogen peroxide present on post-blast debris was not quantified. The HPLC/ED and HPLC/FD analyses were performed as qualitative analyses only.

Table 7.12. Results of analysis of materials collected from a small scale detonation of hydrogen peroxide/nitromethane inside of a car. Hydrogen peroxide residue was detected on just over half of the collected materials.

\begin{tabular}{|c|c|c|c|c|}
\hline Material & \multicolumn{2}{|l|}{$\mathrm{H}_{2} \mathrm{O}_{2}$ by HPLC/ED in DC Mode } & \multicolumn{2}{l|}{$\mathrm{H}_{2} \mathrm{O}_{2}$ by HPLC/FD } \\
\hline Cotton Swabs (7) & No (3) & Yes (4) & No (6) & Yes (1) \\
\hline Glass (2) & No (2) & Yes (0) & No (2) & Yes (0) \\
\hline Witness Plates (6) & No (0) & Yes (6) & No (0) & Yes (6) \\
\hline Seat Fabric (3) & No (2) & Yes (1) & No (3) & Yes (0) \\
\hline Plastic Remnants (4) & No (2) & Yes (2) & No (3) & Yes (1) \\
\hline
\end{tabular}


The data in Table 7.12 show that hydrogen peroxide was not detected on either of the glass samples, and was only detected on one of the pieces of seat fabric by HPLC/ED. The presence of hydrogen peroxide in this sample could not be confirmed by HPLC/FD because the concentration of peroxide in the sample was below the limit of detection for the fluorescence method. Hydrogen peroxide was detected on two of the plastic remnants, but this could only be confirmed by HPLC/FD for one of the samples. Of the extracts from the swabs which were analyzed, four swabs were positive for hydrogen peroxide by HPLC/ED in DC mode, but the presence of hydrogen peroxide could only be confirmed by HPLC/FD in one of the swabs. This swab had been collected from the interior of the car very near to the location of the explosive device. In contrast, hydrogen peroxide was detected on all six witness plates by both analytical methods. The results from the analysis of one of the witness plates are presented in Figures 7.14 and 7.15. Hydrogen peroxide is clearly present in both chromatograms.

As a safety precaution, the interior and exterior of the car were soaked with water prior to placing and detonating the explosive charge. All of the interior surfaces of the car were damp to soaking wet when sampling began. To further complicate matters, midway through the collection of post-blast samples, it began to rain and continued to do so for the next few hours. It is believed that the rain may have diluted or washed away any hydrogen peroxide residues which may have been present, making detection of peroxide difficult to impossible. The delicate nature of hydrogen peroxide may therefore impact how real-world post-blast crime scenes are evaluated. 
Figure 7.14. Analysis of a witness plate exposed to small-scale detonation of hydrogen peroxide/nitromethane by HPLC/ED in DC Mode. Conditions as in Figure 7.12. Peak 1, hydrogen peroxide, Rt 1.925 min. Collected just ahead of the rain, all six witness plates were positive for hydrogen peroxide. The front and back faces of the plates were extracted together.

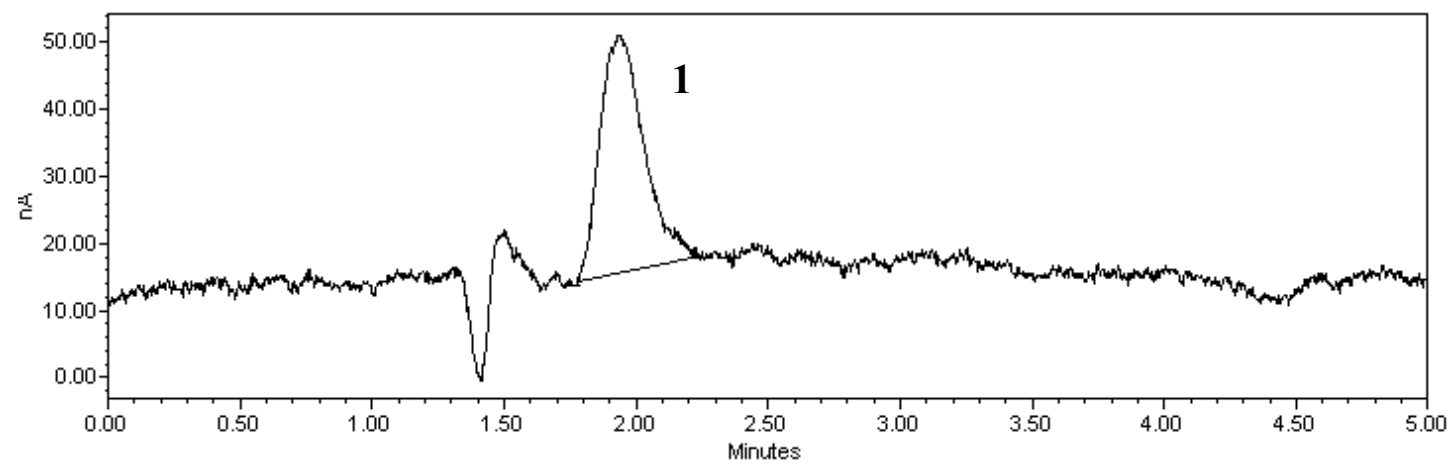

Figure 7.15. Analysis of a witness plate exposed to small-scale detonation of hydrogen peroxide/nitromethane by HPLC/FD. Conditions as in Figure 7.13. Peak 1, hydrogen peroxide, Rt 5.217 min. Collected just ahead of the rain, all six witness plates were positive for hydrogen peroxide. The front and back faces of the plates were extracted together.

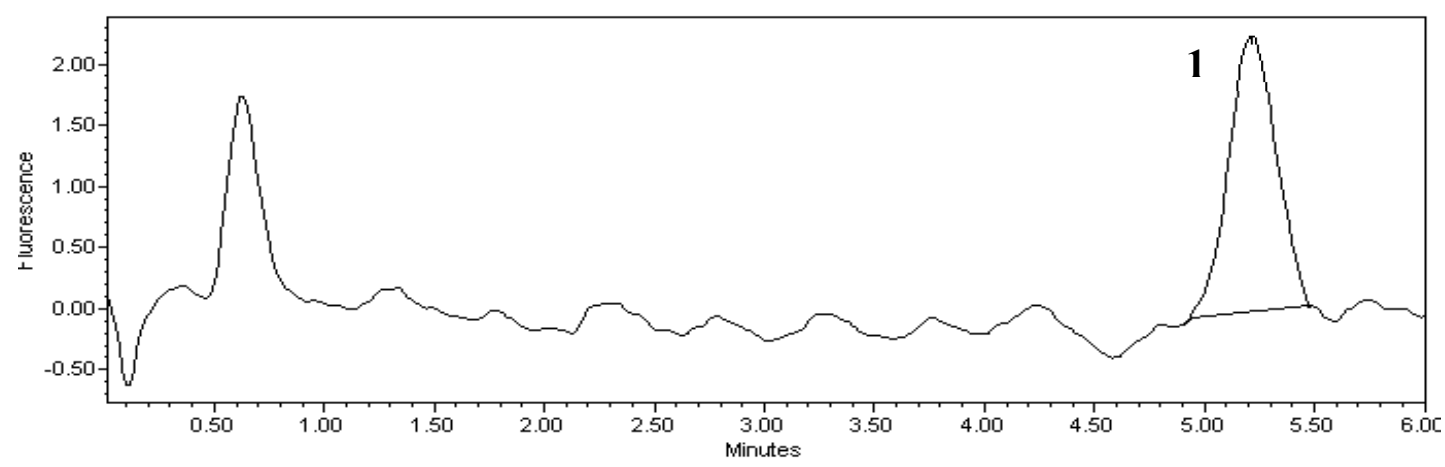


A third controlled detonation of a small-scale mixture of hydrogen peroxide/nitromethane was performed in March of 2009. As in the September 2008 test, the mixture of hydrogen peroxide/nitromethane was contained inside of a metal ammunition can. Post-blast materials from these tests which were collected included a plastic remnant, detonator fragments, ammunition can fragments, and witness plates. Based on the results of the September 2008 test, it was theorized that the ammunition can fragments and the plastic remnants would be the most likely pieces of debris to yield hydrogen peroxide residues. The results of the analyses of the ammunition can fragments, witness plates, and plastic remnants are presented in Table 7.13. Because it is not standard practice at the FBI Laboratory Explosives Unit to quantify post-blast explosives residues, the level of hydrogen peroxide present on post-blast debris was not quantified. The HPLC/ED and HPLC/FD analyses were performed as qualitative analyses only.

Table 7.13. Results of analysis of materials collected from a second small-scale detonation of hydrogen peroxide/nitromethane inside of an ammo can.

\begin{tabular}{|c|c|c|c|c|}
\hline & \multicolumn{2}{|l|}{$\mathrm{H}_{2} \mathrm{O}_{2}$ by HPLC/ED in DC Mode } & $\mathrm{H}_{2} \mathrm{O}_{2}$ by HPLC/FD \\
\hline Plastic Remnants (2) & No (0) & Yes (2) & No (0) & Yes (2) \\
\hline Witness Plates (3) & No (1) & Yes (2) & No (3) & Yes (0) \\
\hline Ammunition Can Fragments (8) & No (0) & Yes (8) & No (0) & Yes (8) \\
\hline Detonator Fragments (2) & No (0) & Yes (2) & No (0) & Yes (2) \\
\hline
\end{tabular}


In viewing Table 7.13 , it is apparent that hydrogen peroxide residues were recovered from all four types of collected materials. Hydrogen peroxide was present on the two witness plates which were closest to the device at the time of detonation, although the amount of residue present was below the limit of detection for the fluorescence method. All of the ammunition can fragments which were collected yielded hydrogen peroxide residues. Two small detonator fragments were recovered. These also yielded hydrogen peroxide. These results are in keeping with the theory that the ammunition can fragments and the plastic remnants were likely to yield hydrogen peroxide residues. The results of the analysis of the plastic remnant are presented in Figures 7.16 and 7.17.

Figure 7.16. Analysis of a plastic remnant by HPLC/ED in DC mode. Conditions: Dionex CarboPac PA10 analytical column, isocratic elution with $150 \mathrm{mM} \mathrm{NaOAc}$ at $\mathrm{pH}$ 10.5, flow rate $1 \mathrm{~mL} / \mathrm{min}, 100 \mu \mathrm{L}$ injection, $\mathrm{T}=45^{\circ} \mathrm{C}$, DC Mode with $\mathrm{E}=+0.4 \mathrm{~V}$. Peak 1, hydrogen peroxide, Rt $1.902 \mathrm{~min}$. The plastic may have been a piece of the container which held the improvised mixture.

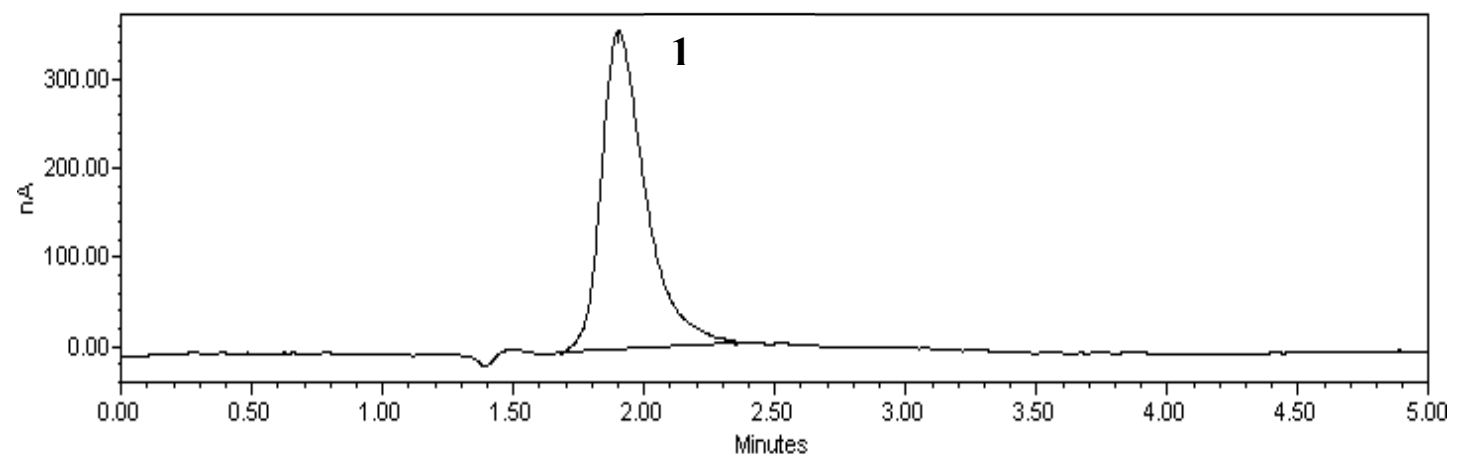


Figure 7.17. Analysis of plastic remnant by HPLC/FD. Conditions: Dionex C-18 analytical column, isocratic elution with $1 \times 10^{-3} \mathrm{M} \mathrm{H}_{2} \mathrm{SO}_{4} / 1 \times 10^{-4} \mathrm{M}$ EDTA at flow rate $0.6 \mathrm{~mL} / \mathrm{min}, 100 \mu \mathrm{L}$ injection, reagent $8 \mu \mathrm{M}$ hemin/80 $\mu \mathrm{M}$ POPHA in ammonia buffer $\mathrm{pH} 9.5$ at flow rate $0.2 \mathrm{~mL} / \mathrm{min}$, post-column reactor $2 \mathrm{~m}, \mathrm{~T}=25^{\circ} \mathrm{C}$, base $0.1 \mathrm{M} \mathrm{NaOH}$ at flow rate $0.2 \mathrm{~mL} / \mathrm{min}, \lambda_{\mathrm{ex}}=320 \mathrm{~nm}, \lambda_{\mathrm{em}}=405 \mathrm{~nm}$. Peak 1 , hydrogen peroxide, Rt 5.195 min. The plastic may have been a piece of the container which held the improvised mixture.

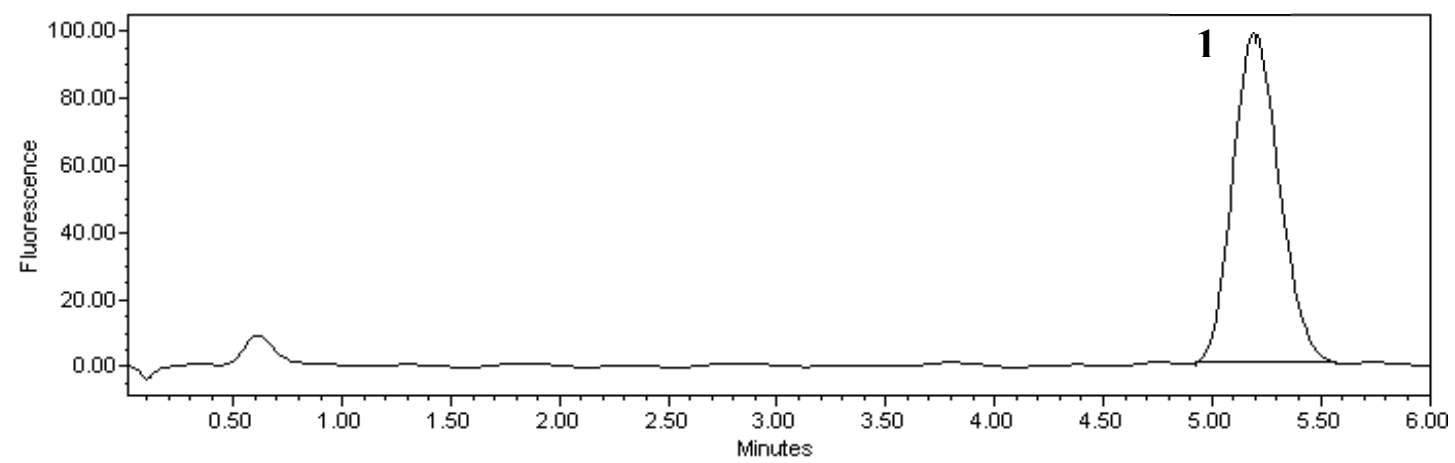

A question arose as to whether the use of cotton swabs to collect post-blast explosives residues would result in the collection of all of the residues which were present on the surface of the debris in a single swabbing, or if additional rounds of swabbing would yield detectable levels of hydrogen peroxide residue. To answer this question, swatches of cotton were used to collect peroxide residues from the exterior and interior of a piece of ammunition can debris. Each face of the debris was swabbed three separate times, and each swab was analyzed on both systems. The peak area of hydrogen peroxide decreased with successive swabs. Interestingly, hydrogen peroxide was detected on both faces of the debris even on the third round of swabbing. It was noted at the time 
of sample collection that the surface of the ammunition can was bent and pitted as a result of exposure to the heat and pressure of an explosion. Hydrogen peroxide residues may have deposited in these areas, resulting in incomplete recovery of hydrogen peroxide from the debris by the cotton on the first and second passes, thereby allowing peroxide to still be recovered on the third pass. These results indicate that swabbing of debris may not permit collection of all of the post-blast residues which are present. Figures 7.18 and 7.19 present the results from the first round of swabbing. It should be noted that this piece of debris contained unusually heavy deposits of hydrogen peroxide.

Figure 7.18. Analysis of $1^{\text {st }}$ dry swab of interior of ammunition can by HPLC/ED in DC mode. Conditions: Dionex CarboPac PA10 analytical column, isocratic elution with 150 $\mathrm{mM} \mathrm{NaOAc}$ at $\mathrm{pH} 10.5$, flow rate $1 \mathrm{~mL} / \mathrm{min}, 100 \mu \mathrm{L}$ injection, $\mathrm{T}=45^{\circ} \mathrm{C}, \mathrm{DC}$ Mode with $\mathrm{E}=+0.4 \mathrm{~V}$. Peak 1, hydrogen peroxide, Rt $1.925 \mathrm{~min}$. Swabbing of post-blast debris may permit the collection of some, but not all, of the explosives residues which are present.

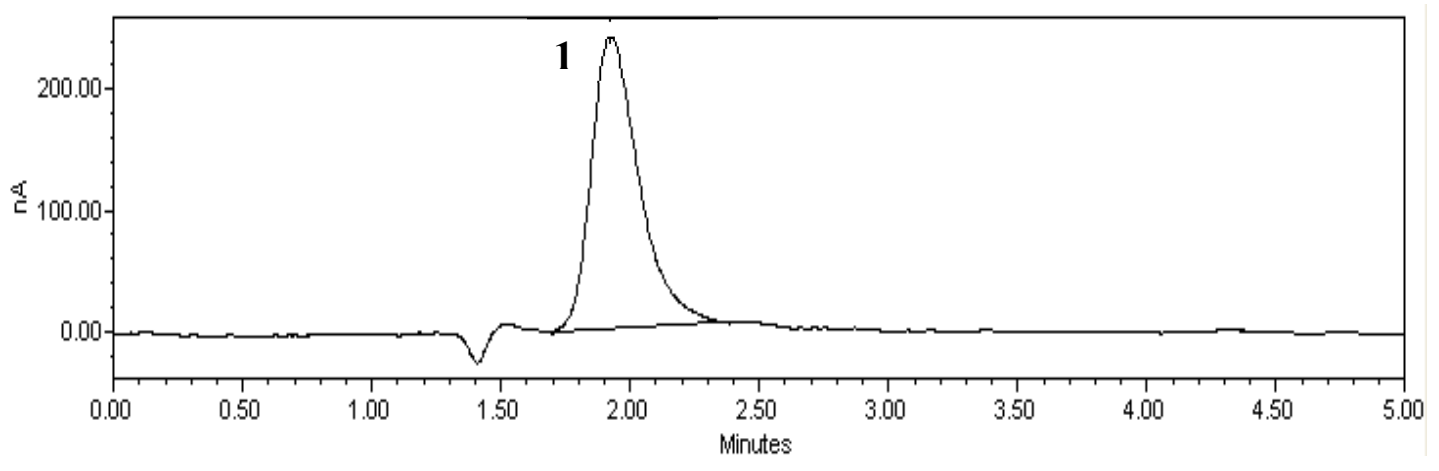


Figure 7.19. Analysis of $1^{\text {st }}$ dry swab of interior of ammunition can by HPLC/FD.

Conditions: Dionex C-18 analytical column, isocratic elution with $1 \times 10^{-3} \mathrm{M} \mathrm{H}_{2} \mathrm{SO}_{4} / 1 \times 10^{-}$

${ }^{4} \mathrm{M}$ EDTA at flow rate $0.6 \mathrm{~mL} / \mathrm{min}, 100 \mu \mathrm{L}$ injection, reagent $8 \mu \mathrm{M}$ hemin $/ 80 \mu \mathrm{M}$ POPHA in ammonia buffer $\mathrm{pH} 9.5$ at flow rate $0.2 \mathrm{~mL} / \mathrm{min}$, post-column reactor $2 \mathrm{~m}$, $\mathrm{T}=25^{\circ} \mathrm{C}$, base $0.1 \mathrm{M} \mathrm{NaOH}$ at flow rate $0.2 \mathrm{~mL} / \mathrm{min}, \lambda_{\mathrm{ex}}=320 \mathrm{~nm}, \lambda_{\mathrm{em}}=405 \mathrm{~nm}$. Peak 1 , hydrogen peroxide, Rt $5.237 \mathrm{~min}$. Swabbing of post-blast debris may permit the collection of some, but not all, of the explosives residues which are present.

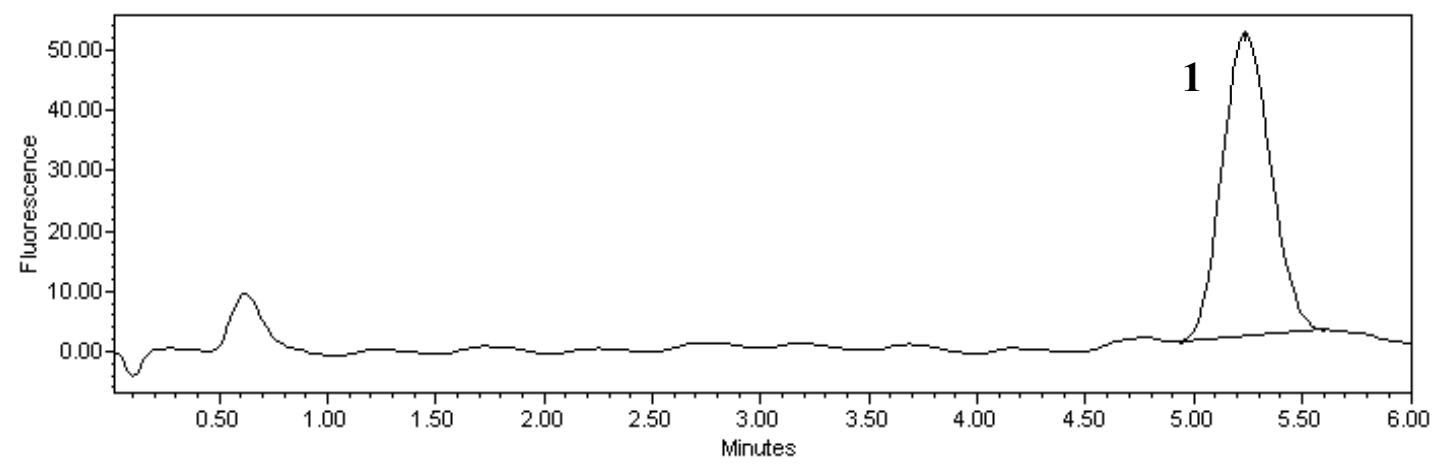

Several conclusions can be drawn from the results of the three field tests involving the detonation of small-scale mixtures of concentrated hydrogen peroxide/nitromethane. The first conclusion that may be drawn is that changing the mode of detection from PAD mode to DC mode and re-optimizing the composition of the mobile phase resulted in a dramatic improvement in the ability to detect hydrogen peroxide residue in post-blast materials by HPLC/ED. The HPLC/ED in DC mode and HPLC/FD methods are both relatively free from interfering compounds. During the course of the analysis of all of the post-blast samples, the majority of the extracts from the post-blast debris only displayed peaks for hydrogen peroxide. Not one sample displayed any additional chromatographic peaks on either the electrochemical or the 
fluorescence systems. Considering the "dirty" nature of post-blast debris, and that no sample clean-up steps were performed other than the filtering of particulates, the lack of interfering matrix components is notable.

Another conclusion that may be drawn is the negative effect the presence of water has on the recovery of hydrogen peroxide residues. In comparison to the test involving the detonation of a small-scale mixture of hydrogen peroxide/nitromethane under dry conditions, the small scale hydrogen peroxide/nitromethane mixture which was detonated inside of a wet car and then rained on was less successful in terms of recovery of peroxide residues from post-blast debris. This result highlights the delicate nature of hydrogen peroxide. Special precautions may need to be taken to successfully collect postblast hydrogen peroxide residues from actual scenes of terrorist or criminal activity.

\section{F. Analysis of Organic Peroxides}

Because the fluorescence and electrochemical systems were optimized for the separation and detection of hydrogen peroxide, it was unknown whether these systems could be employed for the analysis of organic peroxides. In this study, a set of five commercially available peroxides were analyzed on both HPLC systems. This group included urea hydrogen peroxide, dicumyl peroxide, $t$-butyl peroxide, benzoyl peroxide, and cumene hydroperoxide. Urea hydrogen peroxide is also called carbamide peroxide, and it is used as a tooth whitening agent. Dicumyl peroxide and $t$-butyl peroxide are utilized as cross-linking agents in polymer chemistry. Benzoyl peroxide is the active ingredient in many acne medications. Cumene hydroperoxide is used in the synthesis of phenols. The structures of these compounds are presented in Figure 7.20. 
Figure 7.20. Structures of organic peroxides. Each of these compounds is commercially available. It was unknown whether they would interfere with the separation and detection of hydrogen peroxide by HPLC/FD or HPLC/ED in DC or PAD mode.

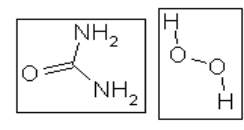

urea hydrogen peroxide

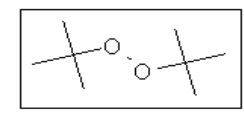

t-butyl peroxide

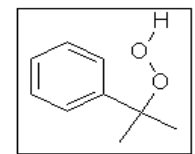

cumene

hydroperoxide

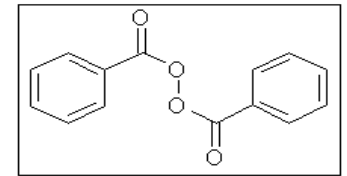

benzoyl peroxide

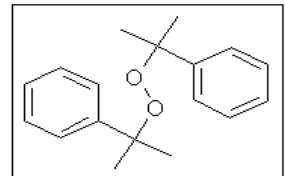

dicumyl peroxide

Each of the organic peroxides was initially tested without the analytical columns in place to determine whether or not they would yield a signal under the standardized detection conditions. The peroxides were initially prepared individually in ethanol; they were then diluted to $100 \mu \mathrm{M}$ in the appropriate mobile phase $\left(0.001 \mathrm{M} \mathrm{H}_{2} \mathrm{SO}_{4} / 0.0001 \mathrm{M}\right.$ EDTA for the samples analyzed on the fluorescence system, and $150 \mathrm{mM} \mathrm{NaOAc}$ for the samples analyzed on the electrochemical system). To ensure that the signal generated on the fluorescence system was only the result of interaction between the organic peroxides and the hemin/POPHA reagent, the peroxides were also tested without the addition of the reagent. For all five organic peroxides as well as for hydrogen peroxide, no signal resulted when the reagent was not present. The results of these experiments are presented in Table 7.14. 
Table 7.14. Results of testing organic peroxides without the presence of the analytical column. Each compound was analyzed at a concentration of $100 \mu \mathrm{M}$ in the appropriate mobile phase for that analytical system.

\begin{tabular}{|c|c|c|c|}
\hline Compound $(100 \mu M)$ & Abbreviation & Ave FD Signal & Ave ED Signal \\
\hline Hydrogen peroxide & HP & $19.4 \times 10^{6}$ & $31.6 \times 10^{3}$ \\
\hline Urea hydrogen peroxide & UHP & $16.7 \times 10^{6}$ & $28.6 \times 10^{3}$ \\
\hline Tert-butyl peroxide & TBP & $8.04 \times 10^{6}$ & $1.05 \times 10^{2}$ \\
\hline Cumene hydroperoxide & CHP & $2.79 \times 10^{6}$ & $7.95 \times 10^{2}$ \\
\hline Benzoyl peroxide & BP & $1.68 \times 106$ & $7.87 \times 10^{2}$ \\
\hline Dicumyl peroxide & DCP & $92.9 \times 10^{3}$ & $5.61 \times 10^{2}$ \\
\hline Water & & $91.3 \times 10^{3}$ & $5.5 \times 10^{1}$ \\
\hline
\end{tabular}

At a concentration of $100 \mu \mathrm{M}$ in the mobile phase, the organic peroxides each yielded a detectable signal on the fluorescence system, with the exception of dicumyl peroxide. As expected, hydrogen peroxide gave the strongest signal on the fluorescence system, followed fairly closely by urea hydrogen peroxide. Tert-butyl peroxide, cumene hydroperoxide, and benzoyl peroxide were also detected on the fluorescence system at a concentration of $100 \mu \mathrm{M}$, though not as strongly as urea hydrogen peroxide. Dicumyl peroxide was not detected on the fluorescence system even at concentrations up to $1 \mathrm{mM}$. In contrast, urea hydrogen peroxide, tert-butyl peroxide, cumene hydroperoxide, and benzoyl peroxide were detected on the fluorescence system at concentrations as low as 10 $\mu \mathrm{M}$. Figure 7.21 represents the signal strength of all six peroxides from strongest to weakest at $100 \mu \mathrm{M}$. There appears to be a trend between steric hindrance and signal 
strength: the more constrained the compound was, the weaker the signal for the reaction product was. This is reasonable when one considers that in order for the fluorescent dimer to be produced, the peroxide moiety had to interact with the hemin enzyme and the $p$-hydroxyphenylacetic acid reagent. Peroxides which were more sterically hindered were less able to interact with the reagent compounds, resulting in production of less (or none) of the fluorescent product.

Figure 7.21. Fluorescence response to peroxides from strongest to weakest. Dicumyl peroxide was not detected on the fluorescence system.

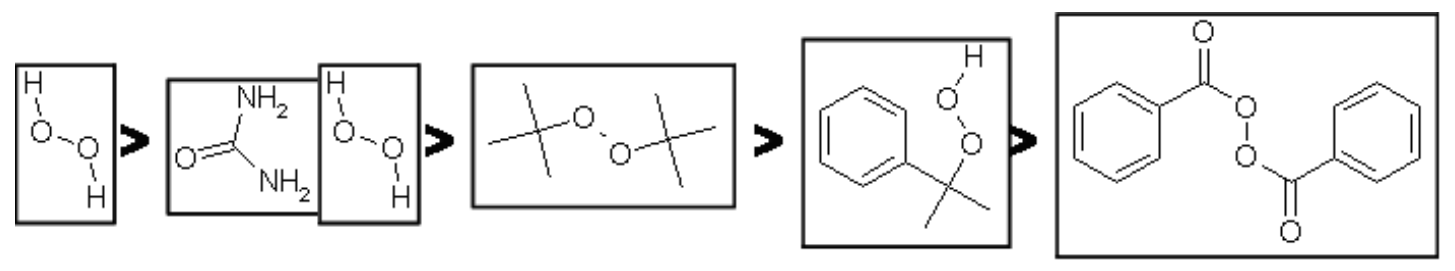

At a concentration of $100 \mu \mathrm{M}$ in mobile phase, the organic peroxides each yielded a detectable signal on the electrochemical system, with the exception of tert-butyl peroxide. As expected, hydrogen peroxide gave the strongest signal on the electrochemical system, followed closely by urea hydrogen peroxide. Dicumyl peroxide, benzoyl peroxide, and cumene hydroperoxide gave electrochemical signals which were almost two orders of magnitude lower than the signal yielded by samples of hydrogen peroxide and urea hydrogen peroxide at the same concentration. Tert-butyl peroxide was essentially undetected at $100 \mu \mathrm{M}$, and its signal strength did not increase at higher concentrations. When the peroxides were re-tested at a concentration of $10 \mu \mathrm{M}$, only hydrogen peroxide and urea hydrogen peroxide yielded a detectable signal. Figure 7.22 
represents the signal strength of all six peroxides from strongest to weakest at $100 \mu \mathrm{M}$. The trend between signal strength and structure is not as clear as for the HPLC/FD method. The electrochemical detection method is affected by steric hindrance to a much lesser degree than is the fluorescence detection method. Rather, it is the ability of the organic peroxide to undergo oxidation at the detection potential of $400 \mathrm{mV}$ that determines signal strength in electrochemical detection.

Figure 7.22. Electrochemical response to peroxides from strongest to weakest. Tert-butyl hydroperoxide was not detected on the electrochemical system in DC mode.

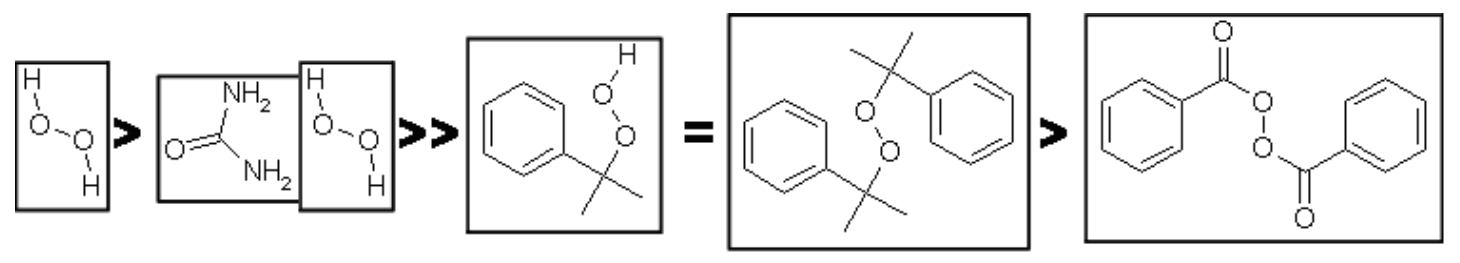

Further analyses of the organic peroxides at a concentration of $100 \mu \mathrm{M}$ in mobile phase were performed with the analytical columns in place. For both the fluorescence and electrochemical methods, only urea hydrogen peroxide was detected. This peak was most likely a result of degradation of urea hydrogen peroxide into hydrogen peroxide, as it showed identical retention time to hydrogen peroxide. In contrast, dicumyl peroxide, benzoyl peroxide, t-butyl peroxide, and cumene hydroperoxide did not elute from either column within sixty minutes of injection. It is likely that these compounds were excessively retained on the column due to a lack of an organic solvent. As discussed earlier, addition of organic solvent to the mobile phase resulted in suppression of the hydrogen peroxide signal. 
During the course of the experiment, the question arose as to whether these organic peroxides might degrade to form hydrogen peroxide. To explore this, the five organic peroxides plus a hydrogen peroxide standard were diluted to a concentration of $100 \mu \mathrm{M}$. They were allowed to sit in a darkened refrigerator for three months, after which time the six peroxides were analyzed. The peroxides were then allowed to sit in a brightly lit room for fourteen days, after which time the peroxides were analyzed again. At each point in the experiment where peroxide analysis was conducted, the samples were tested by HPLC/ED and HPLC/FD without the analytical columns in place to determine the response level of the total peroxide content of the sample. The peroxides were then analyzed with the analytical columns in place to test for the presence of hydrogen peroxide. The results from these studies are presented in Figures 7.23 and 7.24.

Figure 7.23. Analysis of peroxides by electrochemical detection in PAD mode (without analytical columns). Analysis of the samples with the analytical column in place did not reveal the presence of hydrogen peroxide as the organic peroxides decomposed.

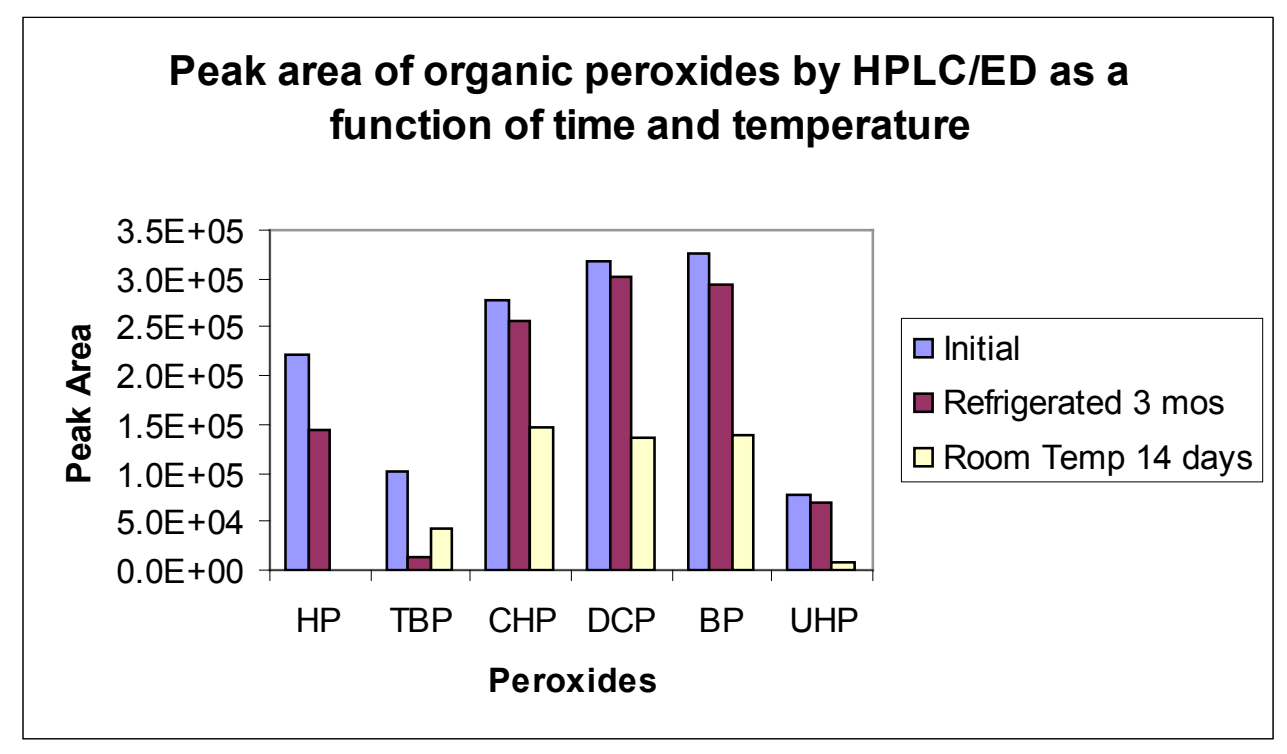


Figure 7.24. Analysis of peroxides by FD (without analytical column). Analysis of the samples with the analytical column in place did not reveal the presence of hydrogen peroxide as the organic peroxides decomposed.

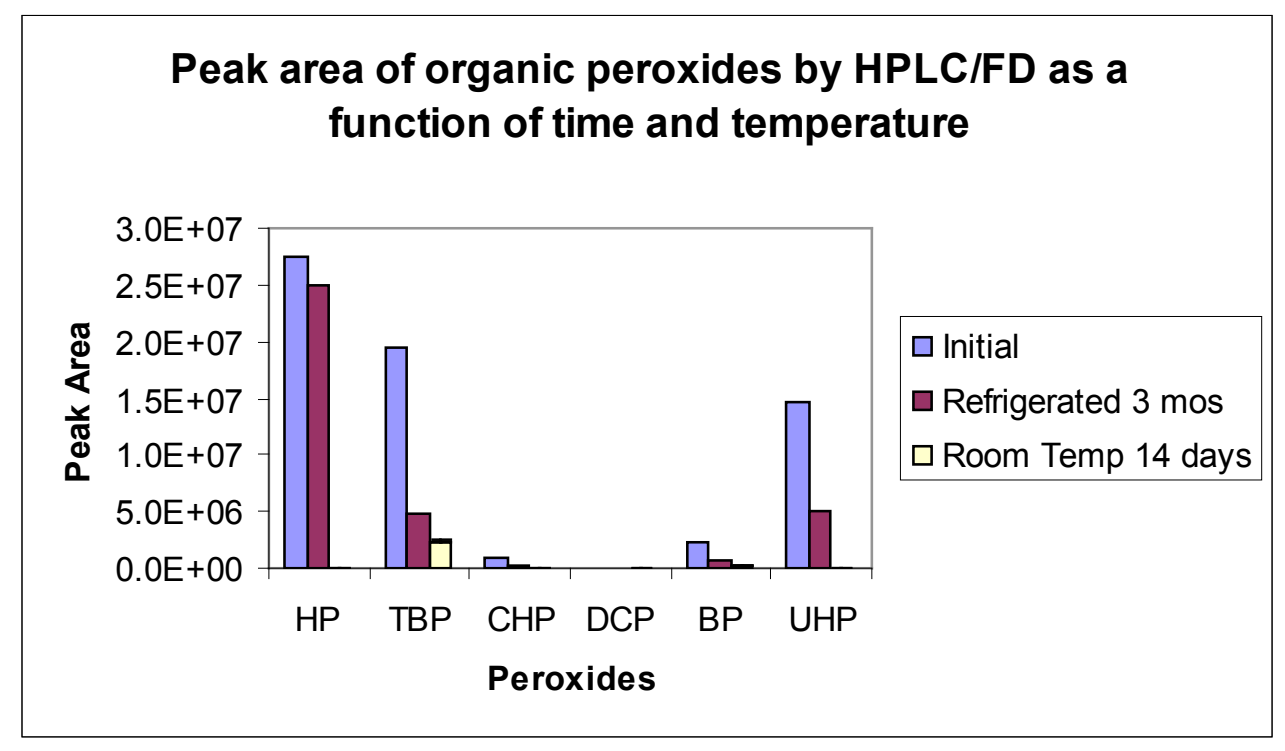

An examination of Figures 7.23 and 7.24 reveal that from the time of the initial analysis until the second analysis three months later, the peak area of all of the organic peroxides and hydrogen peroxides decreased. From the time of the second analysis until the third analysis fourteen days later, the peak area of all of the organic peroxides and hydrogen peroxides decreased further. These data indicate that the organic peroxides were degrading with time. However, it does not appear that the organic peroxides were forming hydrogen peroxide as a degradation product because re-analysis of the samples with the analytical columns in place on both systems did not reveal the presence of any hydrogen peroxide. 


\section{G. Analysis of TATP and HMTD}

Because the fluorescence and electrochemical systems were optimized for the separation and detection of hydrogen peroxide, it was unknown whether these systems could be employed for the analysis of the organic peroxide explosives triacetone triperoxide (TATP) and hexamethylene triperoxide diamine (HMTD). The structures of these compounds are presented in Figure 7.25.

Figure 7.25. Structures of TATP (left) and HMTD (right). Concentrated hydrogen peroxide is a precursor in the manufacturing of both of these explosives.
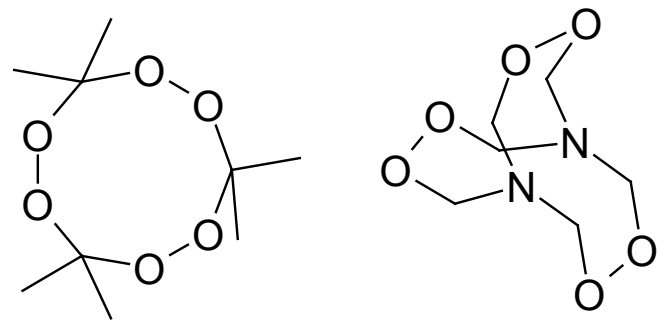

Analysis of samples of intact TATP at concentrations up to $4.5 \mathrm{mM}$ in ethanol failed to yield a detectable signal on either the electrochemical or fluorescence systems, both with and without the presence of the analytical columns. Per Schulte-Ladbeck et al., a UV lamp was employed to photolytically degrade the TATP into hydrogen peroxide (65). However, the addition of this step failed to yield a detectable hydrogen peroxide signal. In contrast, samples of intact HMTD at concentrations up to $4.8 \mathrm{mM}$ in ethanol did yield a hydrogen peroxide signal on both the HPLC/FD and HPLC/ED systems, as well as several additional, unidentified, peaks. These results are presented in Figures 7.26 and 7.27. Given that previous researchers have been able to detect HMTD using 
electrochemical (65) or fluorescence (58) detection only by first degrading the material into hydrogen peroxide, it is unlikely that any of the peaks that were seen in the present analysis actually correspond to intact HMTD. Rather, these peaks most likely correspond to impurities remaining from the synthesis of the HMTD, such as reaction by-products or leftover starting material.

Figure 7.26. Analysis of intact HMTD by HPLC/FD. Conditions: Dionex C-18 analytical column, isocratic elution with $1 \times 10^{-3} \mathrm{M} \mathrm{H}_{2} \mathrm{SO}_{4} / 1 \times 10^{-4} \mathrm{M}$ EDTA at flow rate $0.6 \mathrm{~mL} / \mathrm{min}$, $100 \mu \mathrm{L}$ injection, reagent $8 \mu \mathrm{M}$ hemin $/ 80 \mu \mathrm{M}$ POPHA in ammonia buffer $\mathrm{pH} 9.5$ at flow rate $0.2 \mathrm{~mL} / \mathrm{min}$, post-column reactor $2 \mathrm{~m}, \mathrm{~T}=25^{\circ} \mathrm{C}$, base $0.1 \mathrm{M} \mathrm{NaOH}$ at flow rate 0.2 $\mathrm{mL} / \mathrm{min}, \lambda_{\mathrm{ex}}=320 \mathrm{~nm}, \lambda_{\mathrm{em}}=405 \mathrm{~nm}$. Peak 1, unknown, Rt $4.164 \mathrm{~min}$; Peak 2, hydrogen peroxide, Rt 5.128 min; Peak 3, unknown, Rt 5.661 min; Peak 4, unknown, Rt 6.104 min; Peak 5, unknown, Rt 6.708 min. The unknown peaks may correspond to impurities remaining from the synthesis of the HMTD, such as reaction by-products or leftover starting material.

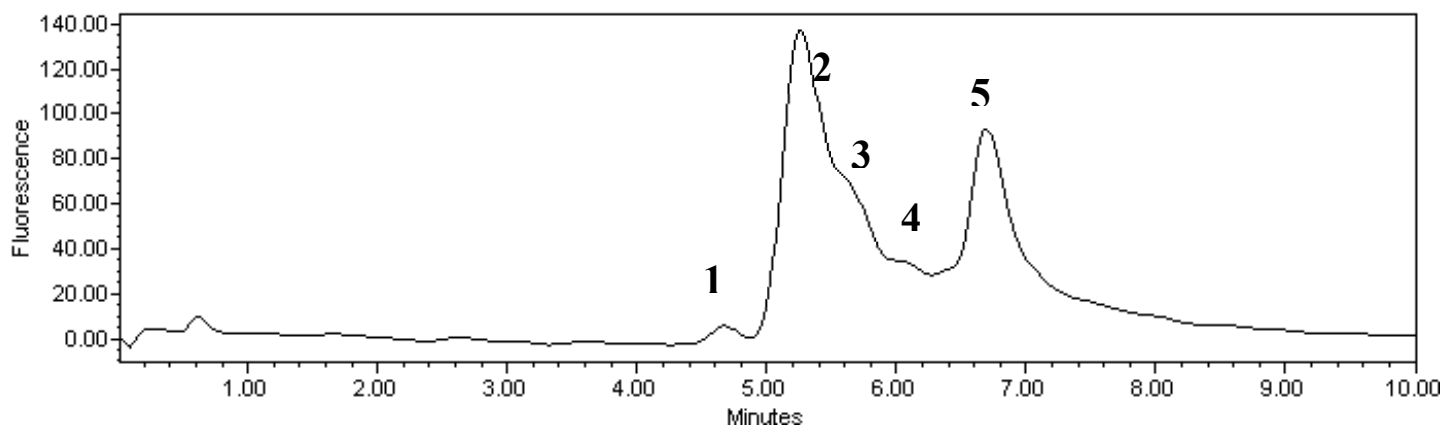


Figure 7.27. Analysis of intact HMTD by HPLC/ED in DC mode. Conditions: Dionex CarboPac PA10 analytical column, isocratic elution with $150 \mathrm{mM} \mathrm{NaOAc} \mathrm{pH} \mathrm{10.5,} \mathrm{flow}$ rate $1 \mathrm{~mL} / \mathrm{min}, 100 \mu \mathrm{L}$ injection, $\mathrm{T}=45^{\circ} \mathrm{C}, \mathrm{DC}$ Mode with $\mathrm{E}=+0.4 \mathrm{~V}$. Peak 1 , unknown, Rt 1.492 min; Peak 2, hydrogen peroxide, Rt 1.912 min. The unknown peak may correspond to an impurity remaining from the synthesis of the HMTD, such as reaction by-products or leftover starting material.

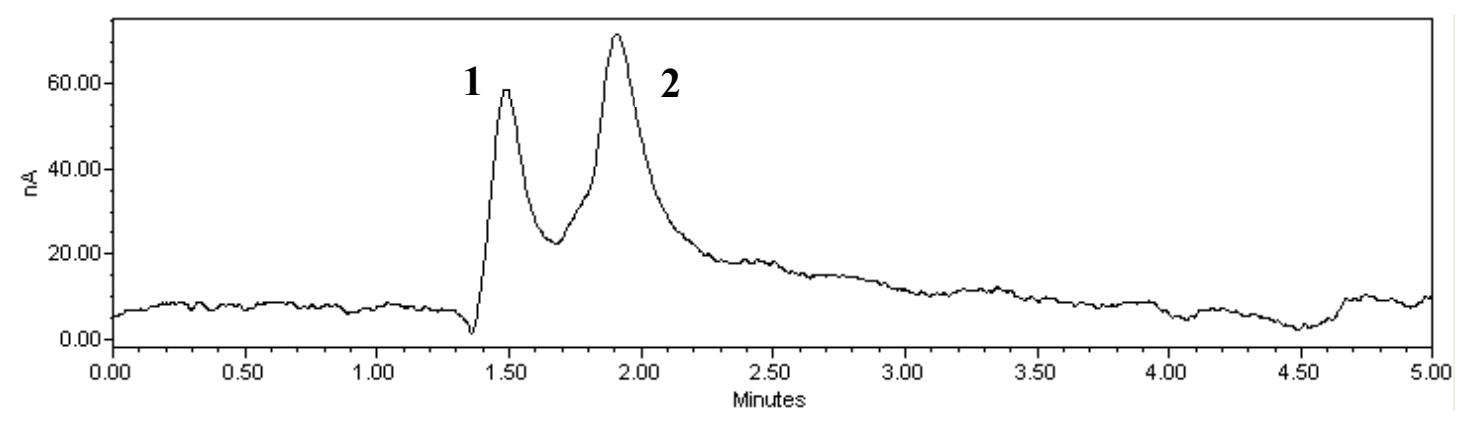

Post-blast TATP residues were extracted from plastic vial fragments which had been collected from the detonation of a small amount of the explosive. The fragments had been packaged in a single layer of Ziploc-type plastic bag, exposed to light, and stored at room temperature for more than one year prior to analysis. The analysis of TATP extract yielded a strong signal for hydrogen peroxide on both analytical systems. This is particularly noteworthy because hydrogen peroxide has not previously been reported as being a component in the post-blast residue of TATP. These are not conditions that had been expected to maintain hydrogen peroxide residues in post-blast debris, yet hydrogen peroxide was nonetheless recovered from the debris. Data from this sample are presented in Figures 7.28 and 7.29. When TATP was burned in a hood under controlled conditions, similar results were noted when the residues were analyzed by HPLC/FD and HPLC/ED 
in DC mode. These results suggest that hydrogen peroxide is a post-blast product of TATP, and not just an impurity left over from the manufacturing process or the result of degradation of the intact TATP. It should be noted that the hydrogen peroxide peak in Figure 7.28 exhibits tailing. The reason for this effect is unknown.

Figure 7.28. Analysis of post-blast residues of TATP by HPLC/ED in DC mode. Conditions as in Figure 7.27. Peak 1, hydrogen peroxide, Rt 1.995 min. Hydrogen peroxide has not previously been noted as a post-blast residue of TATP.

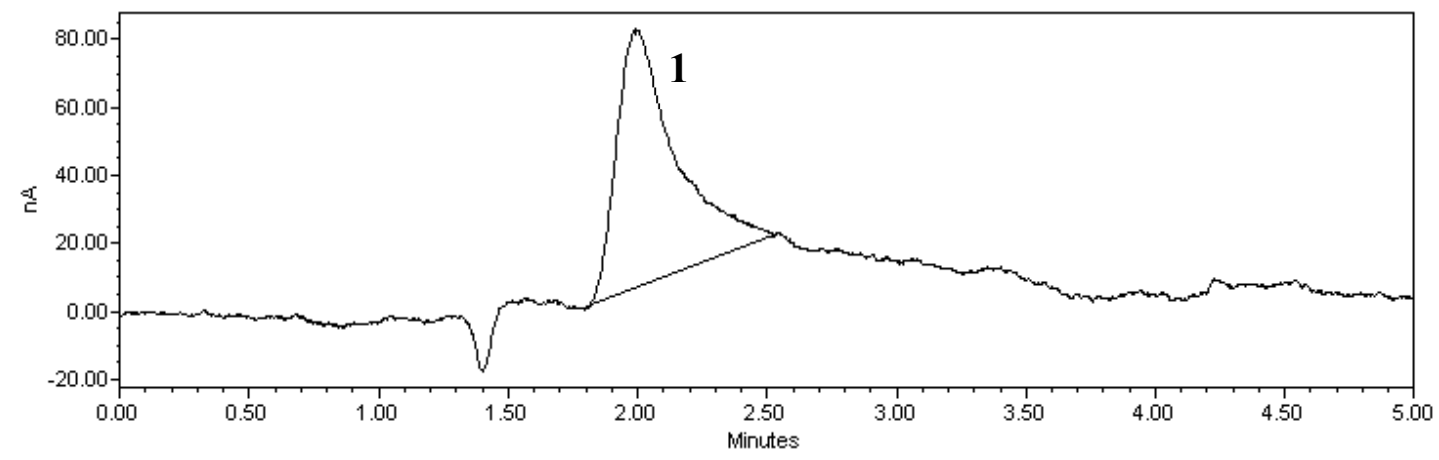

Figure 7.29. Analysis of post-blast residues of TATP by HPLC/FD. Conditions as in Figure 7.26. Peak 1, hydrogen peroxide, Rt 5.217 min. Hydrogen peroxide has not previously been noted as a post-blast residue of TATP.

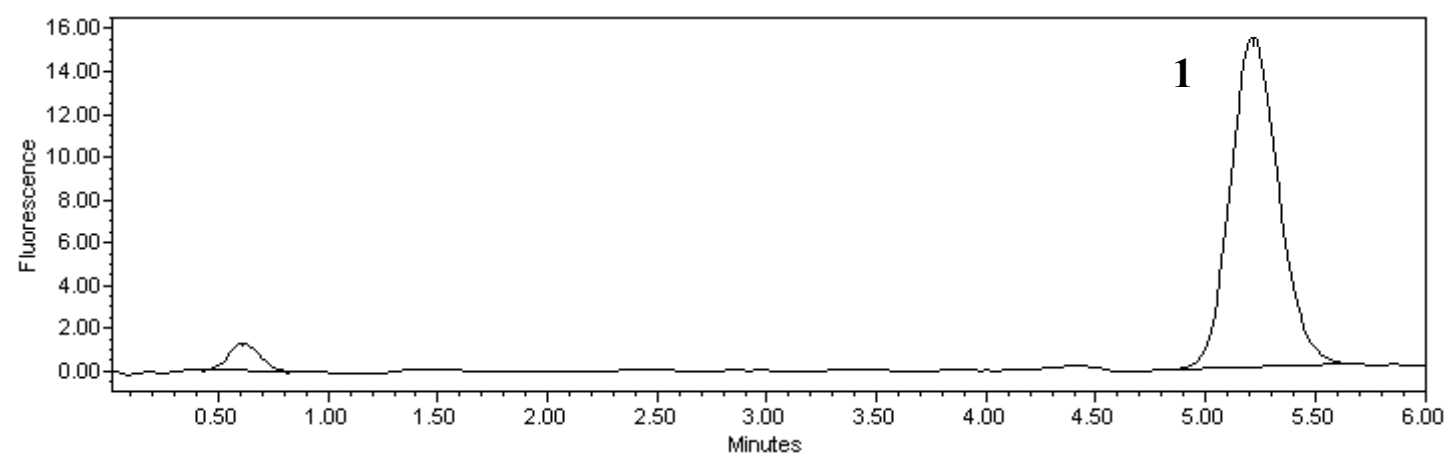


For the analysis of post-burn residues, a small amount of HMTD was ignited on a spatula in the fume hood. The residues were extracted from the spatula in $1.00 \mathrm{~mL}$ of 18.3 M $\Omega$ DI water. Analysis of this residue yielded a single peak at the same retention time as hydrogen peroxide on the HPLC/FD system, as well as three peaks on the HPLC/ED system. These data are presented in Figures 7.30 and 7.31. Although the identity of two of the peaks on the HPLC/ED system is currently unknown, one of the peaks did correspond to the retention time for hydrogen peroxide. It is likely that the unknown peaks may correspond to combustion products of HMTD as they were not present in the pre-blast control.

Figure 7.30. Analysis of post-blast residue of HMTD by HPLC/ED in DC mode. Conditions: Dionex CarboPac PA10 analytical column, isocratic elution with $150 \mathrm{mM}$ NaOAc $\mathrm{pH} 10.5$, flow rate $1 \mathrm{~mL} / \mathrm{min}, 100 \mu \mathrm{L}$ injection, $\mathrm{T}=45^{\circ} \mathrm{C}$, DC Mode with $\mathrm{E}=+$ 0.4V. Peak 1, unknown, Rt 1.526 min; Peak 2, hydrogen peroxide, Rt 1.927 min; Peak 3, unknown, Rt 2.984 min. The unknown peaks may correspond to combustion products of HMTD or to materials remaining from the synthesis of the material.

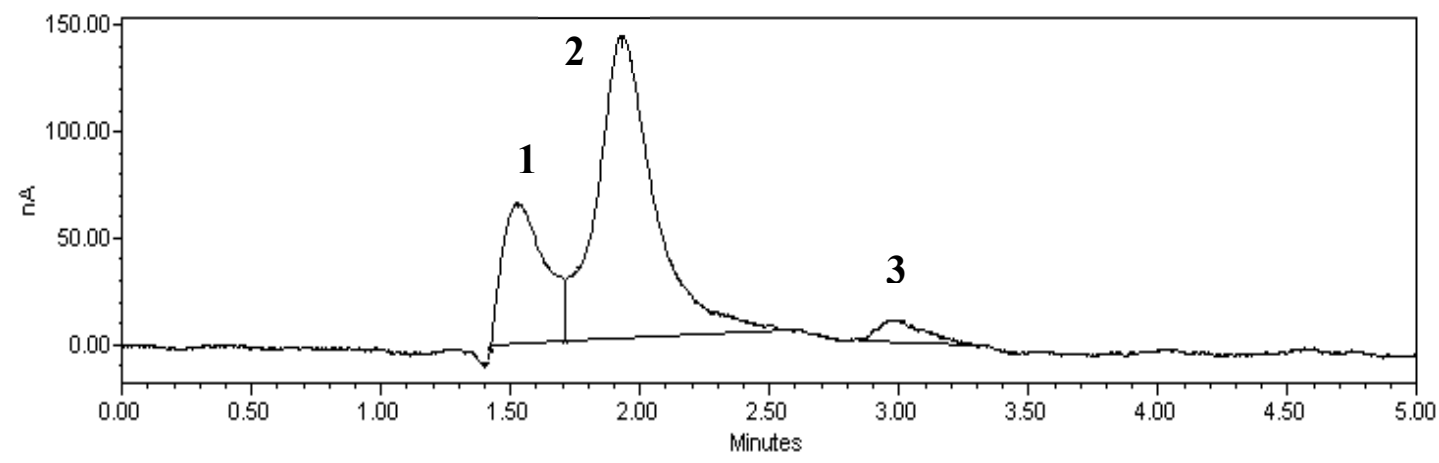


Figure 7.31. Analysis of post-blast residues of HMTD by HPLC/FD. Conditions: Dionex C-18 analytical column, isocratic elution with $1 \times 10^{-3} \mathrm{M} \mathrm{H}_{2} \mathrm{SO}_{4} / 1 \times 10^{-4} \mathrm{M}$ EDTA at flow rate $0.6 \mathrm{~mL} / \mathrm{min}, 100 \mu \mathrm{L}$ injection, reagent $8 \mu \mathrm{M}$ hemin $/ 80 \mu \mathrm{M}$ POPHA in ammonia buffer $\mathrm{pH} 9.5$ at flow rate $0.2 \mathrm{~mL} / \mathrm{min}$, post-column reactor $2 \mathrm{~m}, \mathrm{~T}=25^{\circ} \mathrm{C}$, base $0.1 \mathrm{M}$ $\mathrm{NaOH}$ at flow rate $0.2 \mathrm{~mL} / \mathrm{min}, \lambda_{\mathrm{ex}}=320 \mathrm{~nm}, \lambda_{\mathrm{em}}=405 \mathrm{~nm}$. Peak 1 , hydrogen peroxide, $\mathrm{Rt}$ $5.242 \mathrm{~min}$. Unlike in the analysis of intact HMTD, no extraneous peaks are noted in the HPLC/FD chromatogram of post-blast HMTD.

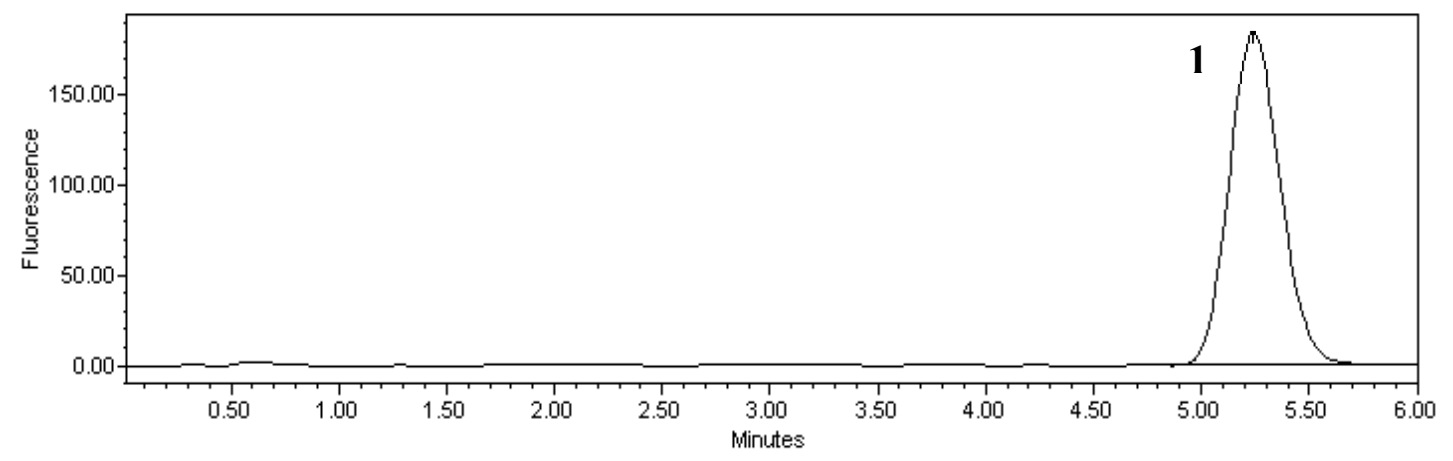

\section{H. Conclusions}

The optimized electrochemical and fluorescence methods for the detection of hydrogen peroxide have been successfully applied to the analysis of post-blast debris from improvised mixtures of concentrated hydrogen peroxide/fuel. Three different mixtures of concentrated hydrogen peroxide with nitromethane were detonated. Hydrogen peroxide residues were detected on post-blast debris from each shot. Experiments indicate that hydrogen peroxide is more readily recovered from metal and plastic post-blast debris than it is from more porous materials, such as fabric or soil. Hydrogen peroxide was also detected in the post-blast residue of TATP and HMTD. This is the first occasion where this phenomenon has been noted. The electrochemical and 
fluorescence methods which were applied to the analysis of hydrogen peroxide appeared to be fairly robust and insensitive to matrix compounds. This is particularly helpful given the notoriously complex nature of post-blast samples.

It is clear that careful choice of swabbing material is important in order to avoid contamination. It was found the hydrogen peroxide is not common in an indoor environment. As a result of the study of the effects of storage conditions on recovery of hydrogen peroxide, it was determined that substrates which contain trace levels of hydrogen peroxide should be stored in refrigerated environments in sealed containers for best results. 


\section{Chapter VIII. Conclusions}

As new explosive threats emerge, it is important to develop analytical techniques and methodologies that can rapidly and reliably identify the nature of those explosives. When pre-blast explosives detection fails or is unavailable, post-blast explosives detection must be employed. The common theme of this research has been the development of analytical methods for the analysis of trace levels of post-blast explosives residue.

The use of ion chromatography with indirect photometric detection allowed for the analysis of a variety of both organic and inorganic anionic species. This type of technology is readily available in many forensic laboratories which perform explosives analysis. Application of the IC-IPD method developed during the course of this research to the analysis of both intact and post-blast samples of ascorbic acid-based black powder substitutes resulted in the separation and detection of the fuel and oxidizer components. The IC-IPD method offered the benefits of a low limit of detection, a linear dynamic range, and demonstrated insensitivity to matrix materials. Future work on the IC-IPD method could be focused on the identification of an analytical column which would permit the simultaneous separation of perchlorate along with ascorbate, chloride, nitrite, nitrate, chlorate, and sulfate for a complete analysis of black powder and black powder substitutes.

In forensic science, it is important that data obtained from the analysis of an unknown sample fit the guidelines which are required for presentation of the data in a court of law. HPLC-ESI-QToFMS is an excellent technique for the analysis of forensic explosives samples because it combines retention time data from the HPLC separation 
with accurate mass measurements, fragmentation information, and isotopic abundance patterns from the mass spectrometric detector. The totality of the data which results from analysis by HPLC-ESI-QToFMS yields a degree of certainty regarding the identity of an unknown analyte which is suitable for use in a court of law. Although few practicing forensic laboratories currently possess such instrumentation, it is believed that the superior resolution of the QToFMS instrument will make it an attractive option in the near future.

The HPLC-ESI-QToFMS method which was optimized for the analysis of the ascorbic acid-based black powder substitutes has been demonstrated to be an excellent tool for the analysis of the organic and inorganic components of these materials. While the analysis time for these samples is rather long at thirty-two minutes, the combination of retention time data, accurate mass measurements, fragmentation information, and isotopic abundance patterns allows for the unequivocal identification of the components present in these propellants. Future work on the HPLC-ESI-QToFMS method could be focused on the determination of the instrument conditions which would permit the detection of ascorbic acid at levels lower than $100 \mu \mathrm{g} / \mathrm{mL}$. This would be very useful in the detection of ascorbic acid in post-blast residue, which would be helpful in identifying the explosive material as originating from an ascorbic acid-based propellant.

As stand-off detection and screening technology for high explosives continue to improve, and as commercial and military high explosives become increasingly difficult for terrorists and criminals to obtain, attention in the explosives community has turned to the peroxide-based explosives. The starting components for the production of these explosives are relatively easy and legal to obtain. While the production of peroxide-based 
explosives is dangerous, it is not particularly difficult. Both solid peroxide-based explosives (TATP and HMTD) and liquid peroxide-based explosives (concentrated hydrogen peroxide/fuel) have been utilized or planned to be utilized in recent terrorist attacks. The development of analytical techniques for the analysis of post-blast peroxide residues is therefore quite important. The HPLC-FD and HPLC-ED methods which have been optimized for the analysis of trace levels of hydrogen peroxide were successfully applied to the analysis of a variety of post-blast substrates. The methods have been demonstrated to be sensitive, selective for hydrogen peroxide, and insensitive to the presence of matrix components. The HPLC-ED method has a wider dynamic range, a more rapid analysis time, and a lower limit of detection in comparison with the HPLCFD method. Another benefit of the electrochemical method is its adaptability. The same combination of detector and analytical column can be applied to the analysis of potential fuel sources, including sugars. In contrast, the HPLC-FD method is more selective than the HPLC-ED method. Because of the nature of the post-column derivatization reaction, the HPLC-FD method will only respond to analytes which contain a peroxide group, while the HPLC-ED method will yield a signal for any analyte which can be oxidized at $\mathrm{E}=+400 \mathrm{~V}$. Future work on the electrochemical and fluorescence techniques could be focused on incorporating fuel analysis with the peroxide analysis.

The methods developed during the course of this research project allow for the analysis of two different types of novel explosives: the low explosive ascorbic acid-based propellants can be analyzed by the techniques of IC-IPD and HPLC-ESI-QToFMS; while the high explosive concentrated hydrogen peroxide/fuel mixtures can be analyzed by HPLC-ED and HPLC-FD. Each set of methods offers unique benefits and drawbacks. 
While additional research could be performed to enhance these benefits and minimize the drawbacks, these methods are sufficiently optimized that they can be utilized as they are for the separation and detection of novel explosives. 


\section{LIST OF REFERENCES}

1. Apodaca, L. Explosives. US Geological Survey 2007 Minerals Yearbook 2007, 1-5.

2. National Counterterrorism Center. 2007 Report on Terrorism;, 2008.

3. Johns, C.; Shellie, R. A.; Potter, O. G.; O'Reilly, J. W.; Hutchinson, J. P.; Guijt, R. M.; Breadmore, M. C.; Hilder, E. F.; Dicinoski, G. W.; Haddad, P. R. Identification of homemade inorganic explosives by ion chromatographic analysis of post-blast residues. Journal of Chromatography A 2008, 1182, 205-214.

4. TWGFEX Laboratory Explosives Group. Recommended guidelines for forensic identification of intact explosives;, 2007.

5. TWGFEX Laboratory Explosives Group. Recommended guidelines for forensic identification of post-blast residues;, 2007.

6. Akhavan, J. Chemistry of Explosives; RSC Paperbacks: Cambridge, 1998.

7. Davis, T. Chemistry of Powder and Explosives; 1941.

8. Midkiff, C. Arson and Explosives Investigation. In Forensic Science Handbook, 2nd ed.; Saferstein, R., Ed.; Prentice Hall: Upper Saddle River, 2001.

9. Oxley, J. What to Detect? In Trace Chemical Sensing of Explosives; Woodfin, R., Ed.; John Wiley \& Sons: Hoboken, 2007.

10. Conkling, J. Chemistry of Pyrotechnics: Basic Principles and Theory; Marcel Dekker Inc: New York, 1985.

11. McCord, B. R.; Bender, E. Chromatography of Explosives. In Forensic Investigations of Explosives; Beveridge, A. D., Ed.; Taylor \& Francis: London, 1998; pp 231-265.

12. Yeager, K. Dangerous Innovations. In Trace Chemical Sensing of Explosives; Woodfin, R., Ed.; John Wiley \& Sons: Hoboken, 2007.

13. Pawlak, D.; Levenson, M. 4128 443, 1978.

14. Bender, E. Analysis of Low Explosives. In Forensic Investigations of Explosives; Beveridge, A. D., Ed.; Taylor \& Francis: London, 1998; pp 231-265. 
15. Roy, M.; W, N. Analysis of dicyandiamide (DCDA), sodium benzoate, and 3nitrobenzoic acid by LC/MS and LC/MS/MS. 8th International Symposium for the Analysis and Detection of Explosives, Ottawa, 2004; pp 433-442.

16. Kurtz, E. 4497 676, 1985.

17. Schulte-Ladbeck, R.; Vogel, M.; Karst, U. Recent methods for the determination of peroxide-based explosives. Analytical and Bioanalytical Chemistry 2006, 386, 559565.

18. CNN. Richard Reid Pleads Guilty., 2003.

19. BBC News. Bomb Plotter Studied Chemistry., 2007.

20. CNN. Tang Bomb: Liquid Explosives are the New Weapon of Choice., 2008.

21. Murphy, P. Report in to the London Terrorist Attacks on 7 July 2005; Intelligence and Security Committee: London, 2006.

22. Occupational Safety and Health Guideline for Hydrogen Peroxide; US Department of Labor.

23. Meyer, R. Explosives; Verlag Chemie: New York, 1977.

24. Wolffenstein, R. Uber die Einwirkung von Wasserstoffsuperoxyd auf Aceton und Mesityloxyd. Berichte der Deutschen Chemischen Gesellschaft 1895, 1895, 22652269.

25. Groth, P. Crystal Structure of 3,3,6,6,9,9-hexamethyl-1,2,4,5,7,8hexaoxacyclononane ("trimeric acetone peroxide"). Acta Chemica Scandinavica 1969, 23, 1311-1329.

26. Jiang, H.; Chu, G.; Gong, H.; Qiao, Q. Tin chloride catalysed oxidation of acetone with hydrogen peroxide to tetrameric acetone peroxide. Journal of Chemical Research 1999, 4, 288-289.

27. Bellamy, A. Triacetone triperoxide: its chemical destruction. Journal of Forensic Sciences 1999, 44, 603-608.

28. Zeman, S.; Trzcinski, W.; Matyas, R. Some properties of explosive mixtures containing peroxides: Part I relative performance and detonation of mixtures with triacetone triperoxide. Journal of Hazardous Materials 2008, 154, 192-198. 
29. Legler, L. Mittheilungen Ueber die sogennante Aeither-oder Lampensaure. Berichte der Deutschen Chemischen GEsellschaft 1881, 14, 602-604.

30. Baeyer, A.; Villiger, V. Mittheilunger Ueber die Nomenclatur der Superoxyde und die Superoxyd der Aldehyde. Berichte der Deutschen Chemischen Gesellschaft 1900, 33, 2479.

31. Skoog, D.; Holler, F.; Nieman, T. Principles of Instrumental Analysis, 5th ed.; Harcourt Brace \& Company: Philadelphia, 1998.

32. Doyle, J.; Miller, M.; McCord, B.; McCollam, D.; Mushrush, G. A multicomponent mobile phase for ion chromatography applied to the separation of anions from the residue of low explosives. Analytical Chemistry 2000, 72, 2302-2307.

33. Paull, B.; Roux, C.; Dawson, M.; Doble, P. Rapid screening of selected organic explosives by high performance liquid chromatography using reversed-phase monolithic columns. Journal of Forensic Sciences 2004, 49, 1181-1186.

34. McCord, B.; Hargadon, K.; Hall, K.; Bermeister, S. Forensic analysis of explosives using ion chromatographic methods. Analytica Chimica Acta 1994, 288, 43-56.

35. Felt, D.; Larson, S.; Escada, L. An extraction/concentration procedure for analysis of low-level explosives in soils. Talanta 2008, 76, 21-28.

36. Perrett, D.; Marchese, S.; Gentili, A.; Curini, R.; Terracciano, A.; Bafile, E. LC-MSMS determination of stabilizers and explosives residues in hand-swabs.

Chromatographia 2008, 68, 517-524.

37. Zeichner, A.; Abramovich-Bar, S.; Tamiri, T.; Almog, J. A feasibility study ont he use of double-sided adhesive coated stubs for sampling of explosives traces from hands. Forensic Science International 2009, 184, 42-46.

38. Lahoda, K.; Collin, O.; Mathis, J.; LeClair, H.; Wise, S.; McCord, B. A survey of background levels of explosives and related compounds in the environment. Journal of Forensic Sciences 2008, 53, 802-806.

39. Popov, I.; Chen, H.; Kharybin, O.; Nikolaev, E.; Cooks, R. Detection of explosives on solid surfaces by thermal desorption and ambient ion/molecule reactions. Chemical Communications 2005, 15, 1953-1955.

40. Thompson, R.; Fetterolf, D.; Miller, M.; Mothershead, R. Aqueous recovery from cotton swabs of organic explosives residue followed by solid phase extraction. Journal of Forensic Sciences 1999, 44, 795-804. 
41. Crowson, A.; Doyle, S.; Todd, C.; Watson, S.; Zolnhofer, N. Quality assurance testing of an explosives trace analysis laboratory - further improvements. Journal of Forensic Sciences 2007, 52, 830-837.

42. Tachon, R.; Pichon, V.; Barbe Le Borgne, M.; Minet, J. Comparison of solid-phase extraction sorbents for sample clean-up in the analysis of organic explosives. Journal of Chromatography A 2008, 1185, 1-8.

43. Ochsenbein, U.; Zeh, M.; Berset, J. Comparing solid phase extraction and direct injection for the analysis of ultra-trace levels of relevant explosives in lake water and tributaries using liquide chromatography electrospray tandem mass spectrometry. Chemosphere 2008, 72, 974-980.

44. Roberts, K.; Haddad, P.; Jackson, P. Principles and Practices of Modern Chromatographic Methods; Elsevier Academic Press: New York, 2004.

45. Small, H.; Stevens, T.; Bauman, W. Novel ion exchange chromatographic method using conductometric detection. Analytical Chemistry 1975, 47, 1801.

46. Fritz, J.; Gjerde, D. Ion Chromatography, 3rd ed.; Wiley VCH: New York, 2000.

47. Kok, W. Principles of Detection. In Handbook of HPLC; Katz, E., Eksteen, R., Schoenmakers, P., Miller, N., Eds.; Marcel Dekker: New York, 1998.

48. Lucy, C.; Krull, I.; Szulic, M. Detection Sensitivity and Selectivity. In Practical HPLC Method Development; Snyder, L., Kirkland, J., Glajch, J., Eds.; John Wiley \& Sons: New York, 1997.

49. Bender, E. The analysis of dicyandiamide and sodium benzoate in pyrodex by HPLC. Crime Lab Digest 1989, 16, 76-77.

50. EPA Method 8330: Nitroaromatics and nitramines by high performance liquid chromatography (HPLC); 1994.

51. Guarav, M.; Rai, P. Solid phase mictroexctration - high performance liquid chromatographic determination of octahydro-1,3,5,7-tetranitro-1.3.5.7-tetrazocine (HMX) and hexahydro-1,3,5-trinitro-1,3,5-triazine (RDX) in the presence of sodium dodecyl sulfate surfactant. Journal of Separation Science 2008, 31, 2173-2181.

52. Wissinger, C.; McCord, B. A gradient reversed phase $\mathrm{HPLCl}$ procedure for smokeless powder comparison. Journal of Forensic Sciences 2002, 47, 168-174. 
53. Verweij, A.; De Bruyne, M.; Klooster, N. Anionenaustauschchromatographie in der analyse von Bombenruckstanden. Archiv fur Krimonologie 1986, 177, 91-94.

54. Bender, E. Indirect photometric detection of anions for the analysis of low explosives. Crime Lab Digest 1989, 16, 78-83.

55. Krull, I.; Szulic, M. Detection Sensitivity and Selectivity. In Practical HPLC Method Development, 2nd ed.; Snyder, L., Kirkland, J., Glajch, J., Eds.; John Wiley \& Sons: New York, 1997.

56. Goodpaster, J.; McGuffin, V. Fluorescence quneching as an indirect detection method for nitrated explosives. Analytical Chemistry 2001, 73, 2004-2011.

57. Meng, H.; Caddy, B. High-performanc eliquid chromatographic analysis with fluroescence detection of ethyl centralite and 2,4-dinitrotoluene in gunshot residue after derivitization with 9-fluorenylmethylchlormoformate. Journal of Forensic Sciences 1996, 41, 213-220.

58. Schulte-Ladbeck, R.; Kolla, P.; Karst, U. Trace analysis of peroxide-based explosives. Analytical Chemistry 2003, 75, 731-735.

59. Hall, K.; McCord, B. The analysis of monvalent and divalent caitons present $\mathrm{i}$ explosives residues using ion chromatography with conductivity detection. Journal of Forensic Sciences 1993, 38, 928-934.

60. Abramovich-Bar, S.; Bamberger, Y.; Ravreby, M.; Levy, S. Applications of ion chromatography for determination and identification of chlorate, nitrite, and nitrate in explosives and explosives residues. 4th Annual Symposium on Analysis and Detection of Explosives, Jerusalem, 1992; pp 41-54.

61. Bozic, R.; West, A.; Levicky, R. Square wave voltammetric detection of 2,4,6trinitrotoluene and 2,4-dinitrotoluene on a gold electrode modified with selfassembled monlayers. Sensors and Actuators B-Chemical 2008, 133, 509-515.

62. Saravanan, N.; Venugopalan, S.; Senthilkumar, N.; Santhosh, P.; Kavita, B.; Prabu, H. Voltammetric determination of nitroaromatic and nitramine explosives contamination in soil. Talanta 2006, 69, 656-662.

63. Matysik, F.; Schumann, U.; Engewalk, W. Isocratic liquid chromatography with segmented columns and simultaneous UV and duel electrochemical detection: application to the selectivity enhancement for the determination of explosives. Electroanalysis 2008, 20, 98-101. 
64. Marple, R.; LaCourse, W. Application of photoassisted electrochemical detection to explosive-containing environmental samles. Analytical Chemistry 2005, 77, 67096714.

65. Schulte-Ladbeck, R.; Karst, U. Liquid-chromatography post-column photochemical conversion and electrochemical detection for determination of peroxide-based explosives. Chromatographia 2003, 57, S61-S65.

66. ESA. Technical Note: The Reference Electrode.

67. Waters 2465 Electrochemical Detector Operator's Guide; Waters Corporation: Milford.

68. Flanagan, R.; Perrett, D.; Whelpton, R. Electrochemical Detection in HPLC: Analysis of Drugs and Poisons; RSC: Cambridge, 2005.

69. Technical Note \#21: Optimal settings for pulsed amperometric detection of carbohydrates using the Dionex ED40 electrochemical detector; Dionex Corporation: Sunnyvale.

70. Technical Note \#20: Analysis of carbohydrates by high performance anion exchange chromatography with pulsed amperometric detection (HPAE-PAD); Dionex Corporation: Sunnyvale.

71. Application Note \#92: Determination of sugars in molasses by high-performance anion exchange with pulsed amperometric detection; Dionex Corporation: Sunnyvale, 2005.

72. Hanko, R.; Rohrer, J. Determination of sucralose in splenda and a sugar-free beverage using high-performance anion-exchange chromatography with pulsed amperometric detection. Journal of Agricultural and Food Chemistry 2004, 52, 4375-4379.

73. Olk, D.; Fortuna, A.; Honeycutt, C. Using anion chromatography-pulsed amperometry to measure amino compounds in dairy manure-amended soils. Soil Science Society of America Journal 2008, 72, 1711-1720.

74. Charoenraks, T.; Chuanuwatanakul, S.; Honda, K.; Yamaguchi, Y.; Chailapakul, O. Analysis of tetracycline antibiotics using HPLC with pulsed amperometric detection. Analytical Sciences 2005, 21, 241-245.

75. Carralero, V.; Gonzalez-Cortes, A.; Yanez-Sedano, P.; Pingarron, J. Pulsed amperometric detection of histamine at glass carbon electrodes modified with gold 
nanoparticles. Electroanalysis 2005, 17, 289-297.

76. Fundamentals: Principles of Mass Spectrometry; Waters Corporation: Milford.

77. Mathis, J.; McCord, B. The analysis of high exlosives by liquid chromagraphy/electrospray ionization mass spectrometry: multiplexed detection of negative ion adducts. Rapid Communications in Mass Spectrometry 2005, 19, 99104.

78. Xu, X.; van de Craats, A.; de Bruyne, P. Highly sensitive screening method for nitroaromatic, nitramine, and nitrate ester explosives by high performance liquid chromatography-atmospheric pressure ionization-mass spectrometry (HPLC-APIMS) in forensic applications. Journal of Forensic Sciences 2004, 49, 1171-1180.

79. Xu, X.; van de Craats, A.; Kok, E.; de Bruyne, P. Trace analysis of peroxide explosives by high performance liquid chromatography-atmospheric pressure chemical ionization-tandem mass spectrometry (HPLC-APCI-MS/MS) for forensic applications. Journal of Forensic Sciences 2004, 49, 1230-1236.

80. Dreifuss, P.; Goodpaster, J. Atmospheric pressure ionization LC/MS methods for the analysis of black powder substitutes. 8th International Symposium on Analysis and Detection of Explosives, Ottawa, 2004; pp 168-180.

81. Norman, W.; Ouderkirk, S. IC-MS detection of inorganic ions of interest in explosives. 9th International Symposium on Analysis and Detection of Explosives, Paris, 2007.

82. Lang, L.; Boyle, K. The analysis of black powder substitutes containing ascorbic acid by ion chromatography/mass spectrometry (IC/MS). 9th International Symposium on Analysis and Detection of Explosives, Paris, 2007.

83. Modi, M.; Crowson, A.; Beardah, M. The use of LC/MS/MS with accmass to analyze peroxide explosives. 9th International Symposium on Analysis and Detection of Explosives, Paris, 2007.

84. Micromass QToF Micro Mass Spectrometer Operator's Guide; Waters Corporation: Milford.

85. Kenny, D.; Pringle, S.; Wildgoose, J. Multi-Push Enhanced Duty Cycle for oa-ToF Mass Spectrometers; Waters MS Technologies Center, 2007.

86. Millar, A.; Castro-Perez, J. Q-ToF Premier: Enhanced Duty Cycle (EDC); Waters Corporation: Milford, 2007. 
87. Hutchinson, J.; Evenhuis, C.; Johns, C.; Kazarian, A.; Breadmore, M.; Macka, M. Identification of inroganic improvised explosive devices by analysis of post-blast residues using portable capillary electrohoresis insturmentation and indirect photometric detection with a light emitting diode. Analycial Chemistry 2007, 79, 7005-7013.

88. Walters, A. Systematic approach to the identification of explosives residues VIII: ascorbic acid containing propellants. 5th International Symposium on Analysis and Detection of Explosives, Washington, DC, 1995.

89. Goodpaster, J.; Keto, R. Identification of ascorbic acid and its degradation products in black powder substitutes. Journal of Forensic Sciences 2004, 49, 523-528.

90. Dreifuss, P.; Klontz, K. Advances in atmospheric pressure ionization (API) LC/MS and GC/MS methods for the analysis and detection of explosives. 7th International Symposium on Analysis and Detection of Explosives, Edinburgh, 2001.

91. Hopper, K.; McCord, B. A comparison of smokeless powders and mixtures by capillary zone electrophoresis. Journal of Forensic Sciences 2005, 50, 307-315.

92. Hopper, K.; LeClair, H.; McCord, B. A novel method for analysis of explosives residues by simultaneous detection of anions and cations via capillary zone electrophoresis. Talanta 2005, 67, 304-312.

93. Hargadon, K.; McCord, B. Journal of Chromatography 1992, 602, 241-247.

94. Kishi, T.; Nakamura, J.; Arai, H. Application of capillary electrophoresis for the determination of inorganic ions in trace explosives and explosive residues. Electrophoresis 1998, 19, 3-5.

95. Nowicki, J.; Pauling, S. Identification of sugars in explosive residues by gas chromatography-mass spectrometry. Journal of Forensic Sciences 1988, 33, 12541261 .

96. Royds, D.; Lewis, S.; Taylor, A. A case study in forensic chemistry: the Bali bombings. Talanta 2005, 67, 262-268.

97. Kuila, D.; Chakrabortty, A.; Sharna, S.; Lahiri, S. Composition profile of low explosives from cases in India. Forensic Science International 2006, 159, 127-131.

98. Sharma, S.; Lahiri, S. Characterization and identification of explosives and exlosives residues using GC-MS and FTIR microscope, and HPTLC. Journal of Energetic Materials 2005, 23, 239-264. 
99. Lim, C.; Chia, P.; Su, W.; Tay, M. Low level detection of low explosives by Raman spectroscopy. 9th International Symposium on Analysis and Detection of Explosives, Paris, 2007.

100. Beveridge, A.; Payton, S.; Audette, R.; Lambertus, A.; Shaddick, R. Systematic analysis of explosive residues. Journal of Forensic Sciences 1975, 20, 431-454.

101. Mahoney, C.; Gillen, G.; Fahey, A. Characterization of gunpowder samples using time-of-flight secondary ion mass spectrometry (TOF-SIMS). Forensic Science International 2006, 158, 39-51.

102. Wang, J.; Pumera, M.; Collins, G.; Opekar, F.; Jelinek, I. A chip-based capillary electrophoresis-contactless conductuvity microsystem for fast measurements of lowexplosive ionic components. Analyst 2002, 127, 719-723.

103. Ding, M.; Chen, P.; Luo, G. Simultaneous determination of organic acids and inorganic anions in tea by ion chromatography. Journal of Chromatography A 1997, 764, 341-345.

104. Alcazar, A.; Fernandez-Caceres, P.; Martin, M.; Pablos, F.; Gonzalez, A. Ion chromatographic determination of some organic acids, chloride, and phosphate in coffee and tea. Talanta 2003, 61, 95-101.

105. Arellano, M.; Andrianary, J.; Dedieu, F.; Couderc, F.; Puig, P. Method development and validation for the simultaneious detection of organic and inorganic acids by capilllary zone electrophoresis. Journal of Chromatography A 1997, 765, 321-328.

106. Schiewe, J.; Mrestani, Y.; Neubert, R. Application and optimization of capillary zone electrophoresis in vitamin analysis. Journal of Chromatography A 1999, 717, 255-259.

107. Versari, A.; Mattioli, A.; Parpinello, G.; Galassi, S. Rapid analysis of ascorbic acid and iso-ascorbic acids in fruit juice by capillary electrophoresis. Food Control 2004, $15,355-358$.

108. Wu, C.; Y, L.; Lee, Y.; Lin, T. Capillary electrophoretic determination of organic acids with indirect detection. Journal of Chromatography A 1995, 716, 291-301.

109. Goodpaster, J. Novel analyses of black powder substitutes and their post-blast residues by reversed phase ESI LC/MS. 17th Sanibel Conference on Mass Spectrometry: Mass Spectrometry in Forensic Science and Counterterrorism, Clearwater, 2005. 
110. Cruz, R.; Vieira, M.; Silva, C. Effect of heat and thermosonication treatments on watercress (Nasturtium offciinale) vitamin $\mathrm{C}$ degradation kinetics. Innovative Food Science and Emerging Technologies 2008, 9, 483-488.

111. Jansson, P.; Jung, H.; Lindqvist, C.; Nordstrom, T. Oxidative decomposition of vitamin $\mathrm{C}$ in drinking water. Free Radical Research 2004, 38, 855-860.

112. Meucci, E.; Martorana, G.; Ursitti, A.; Pischiutta, M.; Miggiano, G.; Castelli, A. Ascorbic acid stability in aqueous solutions. Acta Vitaminologica et Enzymololgica 1985, 7, 147-153.

113. Fay, M.; Bush, M.; Verlangieiri, A. Effect of aldonic acids on the uptake of ascorbic acid by $3 \mathrm{~T} 3$ mouse fibroblasts and human T-lymphoma cells. General

Pharmacology 1994, 25, 1465-1469.

114. Omura, H.; Yamafuji, K. L-ascorbic acid. In Methodicum Chimicum; Korte, K., Goto, M., Eds.; Academic Press: New York, 1977; pp 115-116.

115. Coiffi, N.; Losito, I.; Terzano, R.; Zambonin, C. An electrospray ionization ion trap mass spectrometric (ESI-MS-MSn) study of dehydroascorbic acid hdyrolysis at neutral pH. The Analyst 2000, 125, 2244-2248.

116. Miller, J.; Miller, J. Statistics for Analytical Chemistry, 3rd ed.; Ellis Horwood PTR Prentice Hall: New York, 1993.

117. Bottegal, M.; McCord, B. Analysis of ascorbic acid-based propellants by ion chromatography and capillary electrophoresis. 9th International Symposium on Analysis and Detection of Explosives, Paris, 2007.

118. Gapeev, A.; Sigman, M.; Yinon, J. Liquid chromatographic/mass spectrometric analysis of explosives: RDX adduct ions. Rapid Communications in Mass Spectrometry 2003, 17, 943-948.

119. Krol, J. Determination of perchlorate in water using LC/MS/MS; Waters Corporation: Milford.

120. Waters IC-Pak Column and Guard Column: Care and Use Manual; Waters Corporation: Milford.

121. Lockspray: User's Guide; Micromass: London.

122. Sage, A. Lockspray: Automated Exact Mass Measurements for Electrospray 
Applications; Waters Corporation: Manchester, 2005.

123. Sigman, M.; Armstrong, P. Analysis of oxidizer salt mixtures by electrospray ionization mass spectrometry. Rapid Communications in Mass Spectrometry 2006, 20, 427-432.

124. Zhao, X.; Yinon, J. Forensic identification of explosive oxidizers by electrospray ionization mass spectrometry. Rapid Communications in Mass Spectrometry 2002, $16,1137-1146$.

125. Zhao, X.; Yinon, J. Characterization of ammonium nitrate by electrospray ionization tandem mass spectrometry. Rapid Communications in Mass Spectrometry 2001, 15, 1514-1519.

126. Cioffe, A. 5449 423, 1995.

127. Dillehay, D.; Turner, D.; Blackwell, J. 6361 719, 2002.

128. Griesbach, M.; B, E. 6688 232, 2004.

129. Kurtz, E. 4728 376, 1988.

130. Brauer, B.; Dubnikova, F.; Zeiri, Y.; Kosloff, R.; Gerber, R. Vibrational spectroscopy of triacetone triperoxide (TATP): anharmonic fundamentals, overtones, and combination bands. Spectrochemica Acta Part A 2008, 71, 14381445.

131. Laine, D.; Cheng, I. Electrochemical detection of the explosive hexamethylene diamine (HMTD). Microchemical Journal 2009, 91, 125-128.

132. Germain, M.; Knapp, M. Turn-on fluorescent detection of $\mathrm{H} 2 \mathrm{O} 2$ and TATP. Inorganic Chemistry 2008, 47, 9748-9750.

133. Oxley, J.; Smith, J.; Brady, J.; Dubnikova, F.; Kosloff, R.; Zeiri, L. Raman and infrared fingerprint spectroscopy of peroxide based explosives. Applied Spectroscopy 2008, 62, 906-915.

134. Oxley, J.; Smith, J.; Kirschenbaum, L.; Marimganti, S.; Vadlamannati, S. Detection of explosives in hair using ion mobility spectrometry. Journal of Forensic Sciences 2008, 53, 690-693.

135. Dunayevskiy, I.; Tsekoun, A.; Prasanna, M.; Go, R.; Patel, C. High-sensitivity 
detection of triacetone peroxide and its precursor acetone. Applied Optics 2007, 46, 6397-6404.

136. Sigman, M.; Clark, C.; Fidler, R.; Geiger, C.; Clausen, C. Analysis of triacetone triperoxide by gas chromatography/mass spectrometry and gas chromatography/tandem mass spectrometry by electron and chemical ionization. Rapid Communications in Mass Spectrometry 2006, 20, 2851-2857.

137. Schulte-Ladbeck, R.; Edelmann, A.; Quintas, G.; Lendl, B.; Karst, U. Determination of peroxide-based explosives using liquid chromatography with on-line infrared detection. Analytical Chemistry 2006, 78, 8150-8155.

138. Hage, R.; Lienke, A. Applications of transition-metal catalysts to textile and woodpulp bleaching. Angewandte Chemie International Edition 2005, 45, 206-222.

139. Eul, W.; Moeller, A.; Steiner, N. Hydrogen Peroxide. John Wiley \& Sons, 2001.

140. Material Safety Data Sheet. Hydrogen Peroxide. J.T. Baker: Phillipsburg, 2000.

141. Francois, S.; Sowka, I.; Monod, A.; Temime-Roussel, B.; Laugier, J.; Wortham, H. Development of an online analyzer of atmospheric $\mathrm{H} 2 \mathrm{O} 2$ and several organic hydroperoxides for field campaigns. Atmospheric Research 2005, 74, 525-545.

142. Hellpointner, E.; Gaeb, S. Detection of methyl, hydroxymethyl, and hydroxyethyl hydroperoxides in air and precipitation. Nature 1989, 337, 631-634.

143. Kok, G.; McLaren, S.; Staffelbach, T. HPLC determination of atmospheric organic hydroperoxides. Journal of Atmospheric and Oceanic Technology 1994, 12, 282289.

144. Qi, B.; Zhu, Y.; Hu, M.; Zhang, Y.; Tang, X. Fluorimetric determination of peroxides in an HPLC system. Analytical Letters 2001, 34, 1247-1254.

145. Schulte-Ladbeck, R.; Kolla, P.; Karst, U. A field test for the detection of peroxidebased explosives. Analyst 2002, 127, 1152-1154.

146. Fridovich, I. The stimulation of horseradish peroxidase by nitrogenous ligands. Journal of Biological Chemistry 1963, 238, 3921-3927.

147. Grieshaber, D.; MacKenzie, R.; Voros, J.; Reimhult, E. Electrochemical biosensors sensor principles and architectures. Sensors 2008, 8, 1400-1458. 
148. Osborne, P.; Yamomoto, K. Disposable, enzymatically modified printed fim carbon electrodes for use in the high-performance liquid chromatographic-electrochemical detection of glucose or hydrogen peroxide from immobilized enzyme reactors. Journal of Chromatography B 1998, 707, 3-8.

149. Dupont, F.; Samoil, V.; Chan, R. Extraction of up to $95 \%$ of wheat (Triticum aestivum) flour protein using warm sodium dodecyl sulfate (SDS) without reduction of sonication. Journal of Agricultural and Food Chemistry 2008, 56, 7431-7438.

150. Akiyama, M.; Murakami, K.; Hirano, Y.; Ikeda, M.; Iwatsuki, K.; Wada, A. Characterization of headspace aroma compounds of freshly brewed arabic coffees and studies on a characteristic compound of Ethiopian coffee. Journal of Food Science 2008, 73, C335-C346.

151. Almeida, A.; Braz, D.; Barroso, R.; Lopes, R. Low angle x-ray scattering properties of irradiated spices. Nuclear Instruments and Methods in Physics Research A 2007, 580, 764-767.

152. Portari, G.; Marchini, J.; Jordao, A. Validation of a manual headspace gas chromatography method for determining volatile compounds in biological fluids. Lab Medicine 2008, 39, 42-45.

153. Meaney, M.; McGuffin, V. Investigation of common fluorophores for the detection of nitrated explosives by fluorescence quenching. Analytical Chimica Acta 2008, $610,57-67$.

154. Schreck, A.; Knorr, A.; Wehrstedt, K.; Wandrey, P.; Gmeinwieser, T.; Steinbach, J. Investigation of the explosive hazard of mixtures containing hydrogen peroxide and different alcohols. Journal of Hazardous Materials 2004, 108, 1-7.

155. Ma, W.; Chan, W.; Steinbach, K.; Cai, Z. Determination of five nitrobenzoic acids in groundwater by solid-phase extraction and liquid chromatography-mass spectrometry. Analytical and Bioanalytical Chemistry 2007, 387, 2219-2225.

156. Application Update \#152: An improved gradient method for the AAA-Direct separation of amino acids and carbohydrates in complex sample matrices. Dionex Corporation: Sunnyvale.

157. Installation and operations manual for model LC4B/17 amperometric controller and transducer package. Bioanalytical Systems Inc.: West Lafayette, 1984. 
158. Hwang, H.; Dasgupta, P. Fluorometric flow injection determination of aqueous peroxides at nanomolar level using membrane reactors. Analytical Chemistry 1986, $58,1521-1524$.

159. Francois, S.; Lazrus, A.; Lok, G.; Lind, J.; Gitlin, S.; Heikes, B. Automated fluorimetric method for hydrogen peroxide in air. Analytical Chemistry 1986, 58, 594-597.

160. Guerra, P.; Lai, H.; Almirall, J. Analysis of the volatile chemical markers of explosives using novel solid phase microextraction couple to ion mobility spectrometry. Journal of Separation Science 2008, 31, 2891-2898.

161. Walker, C.; Cullum, H.; Hiley, R. An environmental survey relating to improvised and emulsion/gel explosives. Journal of Forensic Sciences 2001, 46, 254-267. 
VITA

MEGAN NICOLE BOTTEGAL

March 25, 1981

2003

2007

2007

2007-2009

2009-present
Born, Pittsburgh, Pennsylvania

B.S., Chemistry, Biochemistry Track

University of North Carolina at Chapel Hill

Chapel Hill, North Carolina

GSA Scholarly Forum Winner

Florida International University

Miami, Florida

Collaborating Scientist

Bureau of Alcohol, Tobacco, and Firearms

Beltsville, Maryland

Visiting Scientist

Federal Bureau of Investigation Laboratory

Quantico, Virginia

Counterfeit Specialist

United States Secret Service

Washington, DC

\section{PUBLICATIONS AND PRESENTATIONS}

Bottegal M, McCord B. Fire in the hole! Analysis of ascorbic acid-based propellants by ion chromatography and capillary electrophoresis. 9th ISADE, 2007 July 2-5; Paris. 2007.

Bottegal M, Sherlach K, Mount K, McCord B, Miller M. Analysis of trace hydrogen peroxide by HPLC-ED and HPLC-FD. AAFS Meeting, 2009 February 16-21; Denver, CO, 2009.

Bottegal M, Lang L, Miller M, McCord B. Analysis of ascorbic acid-based black powder substitutes by high performance liquid chromatography / electrospray ionization / quadrupole time-of-flight mass spectrometry. In Press. Rapid Communications in Mass Spectrometry. 